Characterisation of genotypic and tissue specific resistance in oilseed rape (B. napus) against Sclerotinia sclerotiorum

\author{
Dissertation \\ to obtain the doctoral degree (Dr. sc. agr.) \\ of the Faculty of Agricultural Sciences, \\ Georg-August-Universität Göttingen, Germany
}

Antonia Wilch

Born in Osterode am Harz, Germany

Göttingen, December 2018 
1. Referee: Prof. Dr. Andreas von Tiedemann

2. Referee: Prof. Dr. Petr Karlovsky

Date of disputation: February 2019 


\section{Meiner Familie}




\section{Index}

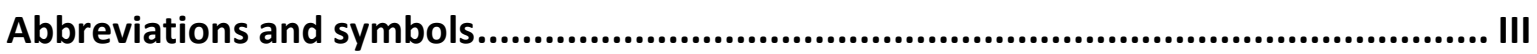

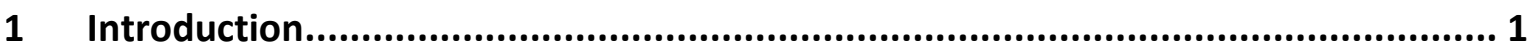

1.1 Oilseed rape production and use in Germany ............................................ 1

1.2 Sclerotinia sclerotiorum: life cycle and consequences of infection................... 3

1.3 Quantitative disease resistance in B. napus against $S$. sclerotiorum ................ 9

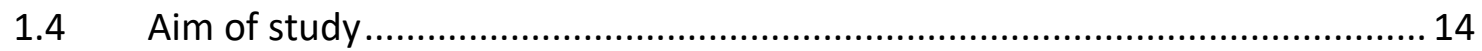

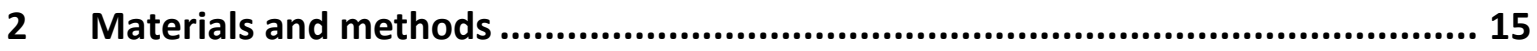

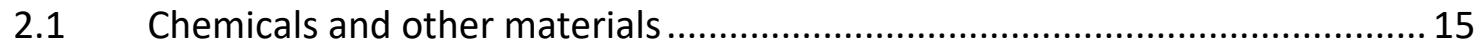

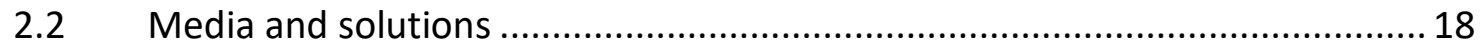

2.3 Plant materials and cultivation for greenhouse experiments ......................... 22

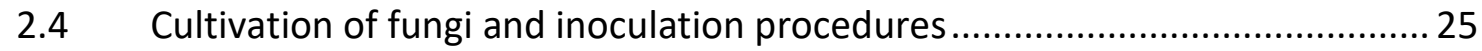

2.5 Disease assessment in greenhouse experiments ........................................ 27

2.6 Quantification of DNA of S. sclerotiorum in plant tissues ................................ 27

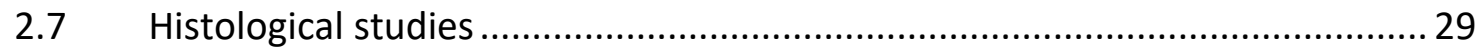

2.7.1 Sampling and fixation of plant material ......................................................... 29

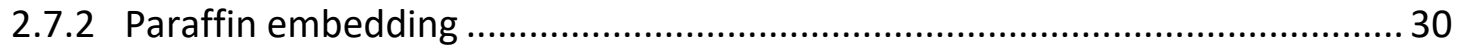

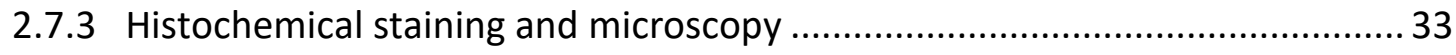

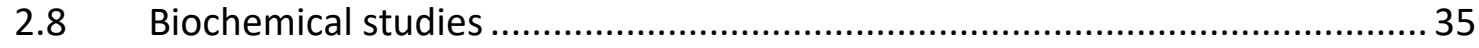

2.8.1 Sampling and preparation of plant material ................................................. 35

2.8.2 Quantification of phenolic acids by HPLC ..................................................... 35

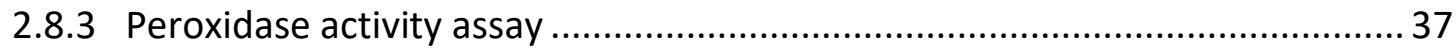

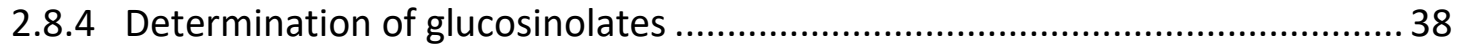

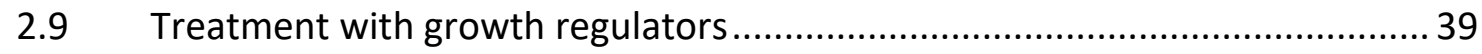

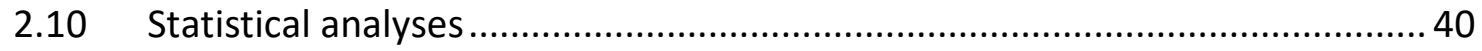

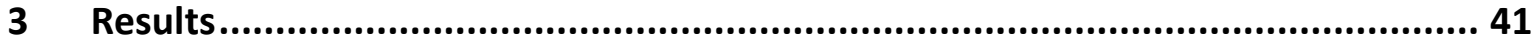

3.1 Screening Brassica germplasm for genotypic resistance by stem inoculation 41

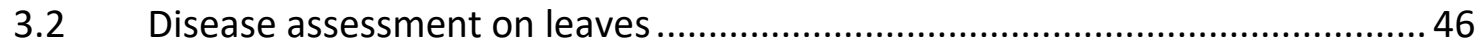

3.3 Disease assessment and fungal development on different plant tissues ........ 48

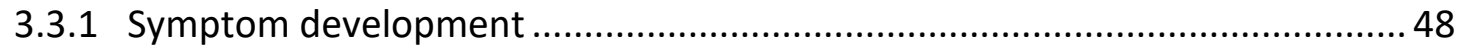

3.3.2 Quantification of DNA of S. sclerotiorum ................................................... 52

3.4 Histological studies on different plant tissues .............................................. 53

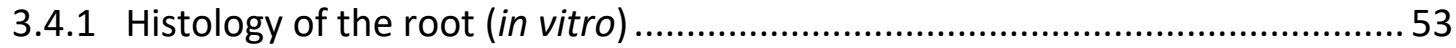

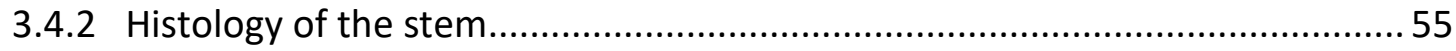




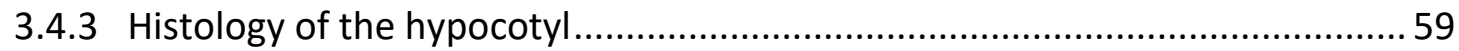

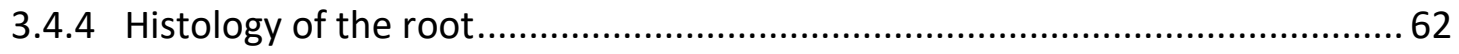

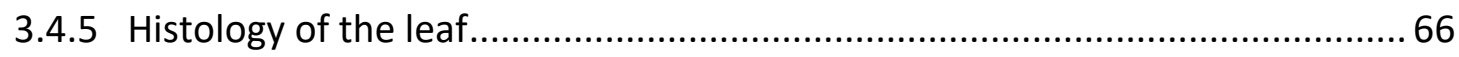

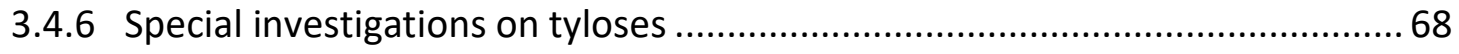

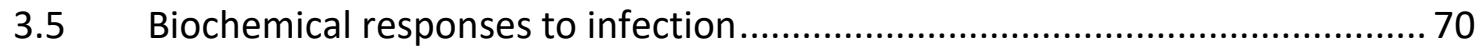

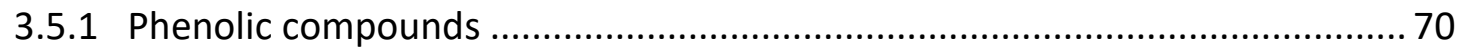

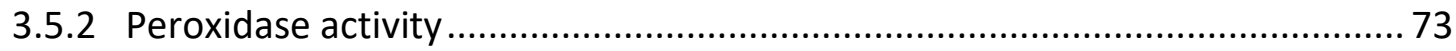

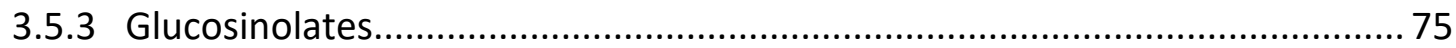

3.6 Effect of growth regulators on development of plants and disease ............... 83

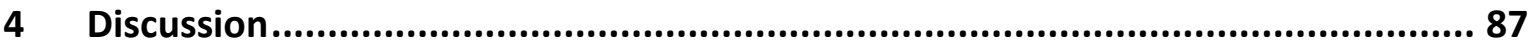

4.1 Lack of complete genotypic resistance against S. sclerotiorum ..................... 87

4.2 Alternative methods for disease assessment............................................... 92

4.3 Disease development in different plant tissues .......................................... 93

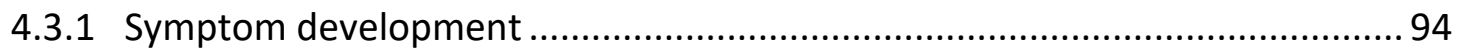

4.3.2 Quantification of fungal colonisation ........................................................... 96

4.4 Infection patterns of S. sclerotiorum in different plant tissues....................... 97

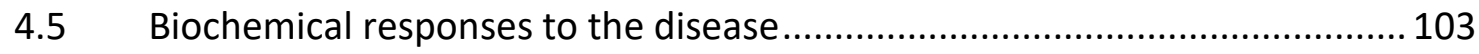

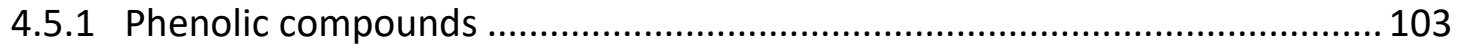

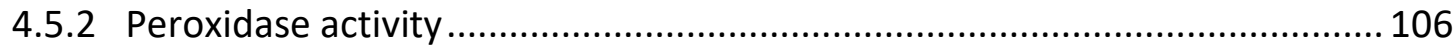

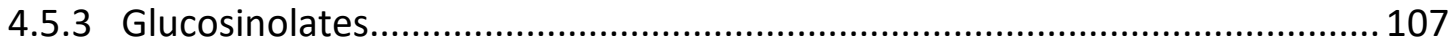

4.6 Effect of growth regulators on development of plants and disease.............. 114

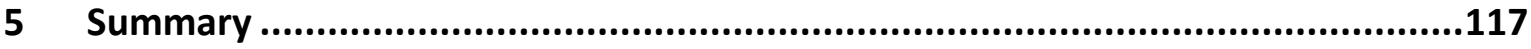

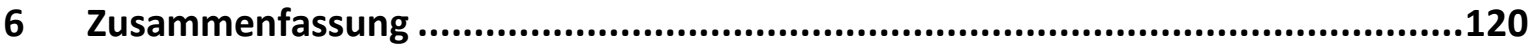

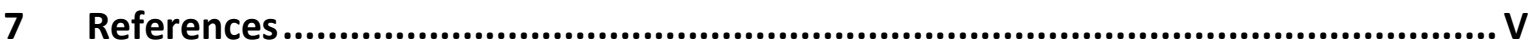

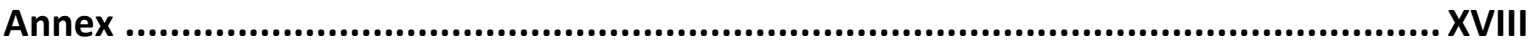

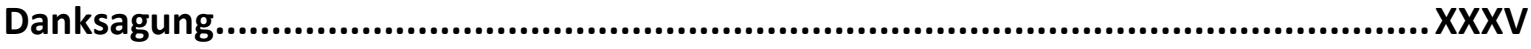

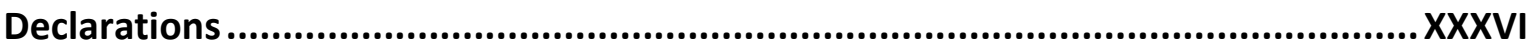




\section{Abbreviations and symbols}

\begin{tabular}{|c|c|}
\hline$\%$ & Percent \\
\hline$</>$ & smaller/bigger than \\
\hline \pm & More or less \\
\hline${ }^{\circ} \mathrm{C}$ & Degree Celsius \\
\hline$\varnothing$ & Diameter \\
\hline$\mu l$ & Microlitre \\
\hline$\mu \mathrm{m}$ & Micrometre \\
\hline$\mu \mathrm{mol}$ & Micromole \\
\hline AFE & Acetic acid-formaldehyde-ethanol solution \\
\hline Al & Active ingredient \\
\hline B & Boron \\
\hline BnaDFFS & Brassica napus Diversity Fixed Foundation Set \\
\hline $\mathrm{BP}$ & Band pass \\
\hline $\mathrm{Ca}^{2+}$ & Calcium ion \\
\hline CBE & Chlorazol black E \\
\hline CLSM & Confocal laser scanning microscope \\
\hline $\mathrm{cm}$ & Centimetre \\
\hline CTAB & Cetyltrimethylammonium bromide \\
\hline $\mathrm{Ctl}$ & Control \\
\hline $\mathrm{Cu}$ & Copper \\
\hline CWDES & Cell wall degrading enzymes \\
\hline DBC & Butylhydroxyanisol \\
\hline df & Degree of freedom \\
\hline $\mathrm{dH}_{2} \mathrm{O} / \mathrm{dd} \mathrm{H}_{2} \mathrm{O}$ & Distilled / bi-distilled water \\
\hline DNA & Deoxyribonucleic acid \\
\hline dpi & Days post inoculation \\
\hline DW & Dry weight \\
\hline e.g. & for example (exempli gratia) \\
\hline EDTA & Ethylenediaminetetraacetic acid \\
\hline $\mathrm{Fe}$ & Iron \\
\hline Fig. & Figure \\
\hline FW & Fresh weight \\
\hline g & Gram \\
\hline GER & Germany \\
\hline h & Hours \\
\hline ha & Hectare \\
\hline $\mathrm{H}_{2} \mathrm{O}_{2}$ & Hydrogen peroxide \\
\hline $\mathrm{HCl}$ & Hydrochloric acid \\
\hline hpi & Hours post inoculation \\
\hline HPLC & High performance liquid chromatography \\
\hline hz & Hertz \\
\hline i.e. & That is (from lat.: id est) \\
\hline
\end{tabular}




\begin{tabular}{|c|c|}
\hline $\mathrm{KH}_{2} \mathrm{PO}_{4}$ & Potassium dihydrogen phosphate \\
\hline 1 & Litre \\
\hline LP & Long pass \\
\hline M & Molar \\
\hline$m^{2}$ & Square metre \\
\hline $\min$ & Minute \\
\hline $\mathrm{ml}$ & Millilitre \\
\hline $\mathrm{mM}$ & Milli molar \\
\hline $\mathrm{mm}$ & Millimetre \\
\hline $\mathrm{Mn}$ & Manganese \\
\hline Mo & Molybdenum \\
\hline Mt & Million tons \\
\hline mTorr & Millitorr \\
\hline NADPH & Nicotinamide adenine dinucleotide phosphate \\
\hline $\mathrm{NaOH}$ & Sodium hydroxide \\
\hline $\mathrm{nm}$ & Nanometre \\
\hline ns & Not significant \\
\hline OD & Optical density \\
\hline OSR & Oilseed rape \\
\hline PAL & Phenylalanine ammonia lyase \\
\hline PAS & Periodic acid Schiff's \\
\hline PBS & Phosphate buffer solution \\
\hline PDA & Potato dextrose agar \\
\hline POX & Peroxidase \\
\hline PPP & Phenylpropanoid pathway \\
\hline QDR & Quantitative disease resistance \\
\hline qPCR & Quantitative real-time polymerase chain reaction \\
\hline QTL & Quantitative trait loci \\
\hline ROS & Reactive oxygen species \\
\hline rpm & Rounds per minute \\
\hline RT & Room temperature \\
\hline s & Second \\
\hline Sc & S. sclerotiorum inoculated \\
\hline SD & Standard deviation \\
\hline SE & Standard error \\
\hline $\mathrm{SO}_{2}$-water & Sulphur dioxide water \\
\hline SPB & Sodium phosphate buffer \\
\hline SSR & Sclerotinia stem rot \\
\hline Tab. & Table \\
\hline U & Units \\
\hline USA & United States of America \\
\hline W & Watt \\
\hline $\mathrm{Zn}$ & Zinc \\
\hline Zy821 & Zhongyou 821 \\
\hline
\end{tabular}




\section{Introduction}

\subsection{Oilseed rape production and use in Germany}

Oilseed rape (Brassica napus L.) is an allotetraploid crop derived from natural hybridisation of the diploid progenitor species B. oleracea and B. rapa. In Germany, oilseed rape (OSR) was already cultivated in the $18^{\text {th }}$ century (Christen \& Friedt, 2007) but only to a very small extent. It was appreciated as a valuable preceding crop, however, due to anti-nutritional substances such as erucic acid and glucosinolates it was mainly used for technical purposes. Only in 1963, when Canadian scientists found mutants characterised by low quantities of erucic acid, production increased. From then on, intensive investigations for enhancement of the crop were made also in Germany which in 1973 finally resulted in the first erucic acid free winter OSR cultivar Lesira ('0-cultivar'). When in 1969 the polish cultivar Bronowski was identified to contain only a low amount of glucosinolates, Bronowski was used to mate with 0-cultivars followed by the breeding of 'double-low cultivars' in the 1980s, now being erucic acid free and containing low amounts of glucosinolates. This progress allowed the expansion of the different ways of use for OSR, leading to a stronger increase of the production as well in Germany (Fig. 1) as worldwide (FAOSTAT, 2018).

The major oilseed rape producing countries are Canada, China, India, France, Germany and Australia producing $80 \%$ of the total worldwide production. In 2016 , the production per year in these countries ranged between $18 \mathrm{Mt}$ in Canada, $15 \mathrm{Mt}$ in China, and a lower production especially due to smaller cultivation area in India, France, Germany and Australia (7 Mt, $5 \mathrm{Mt}, 4.5 \mathrm{Mt}$ and $3 \mathrm{Mt}$, respectively). All in all, rapeseed oil is a high-quality vegetable oil among others. Palm oil and soybean oil are leading the worldwide production with $65 \mathrm{Mt}$ and $55 \mathrm{Mt}$, respectively, followed by rapeseed oil with $28 \mathrm{Mt}$. 


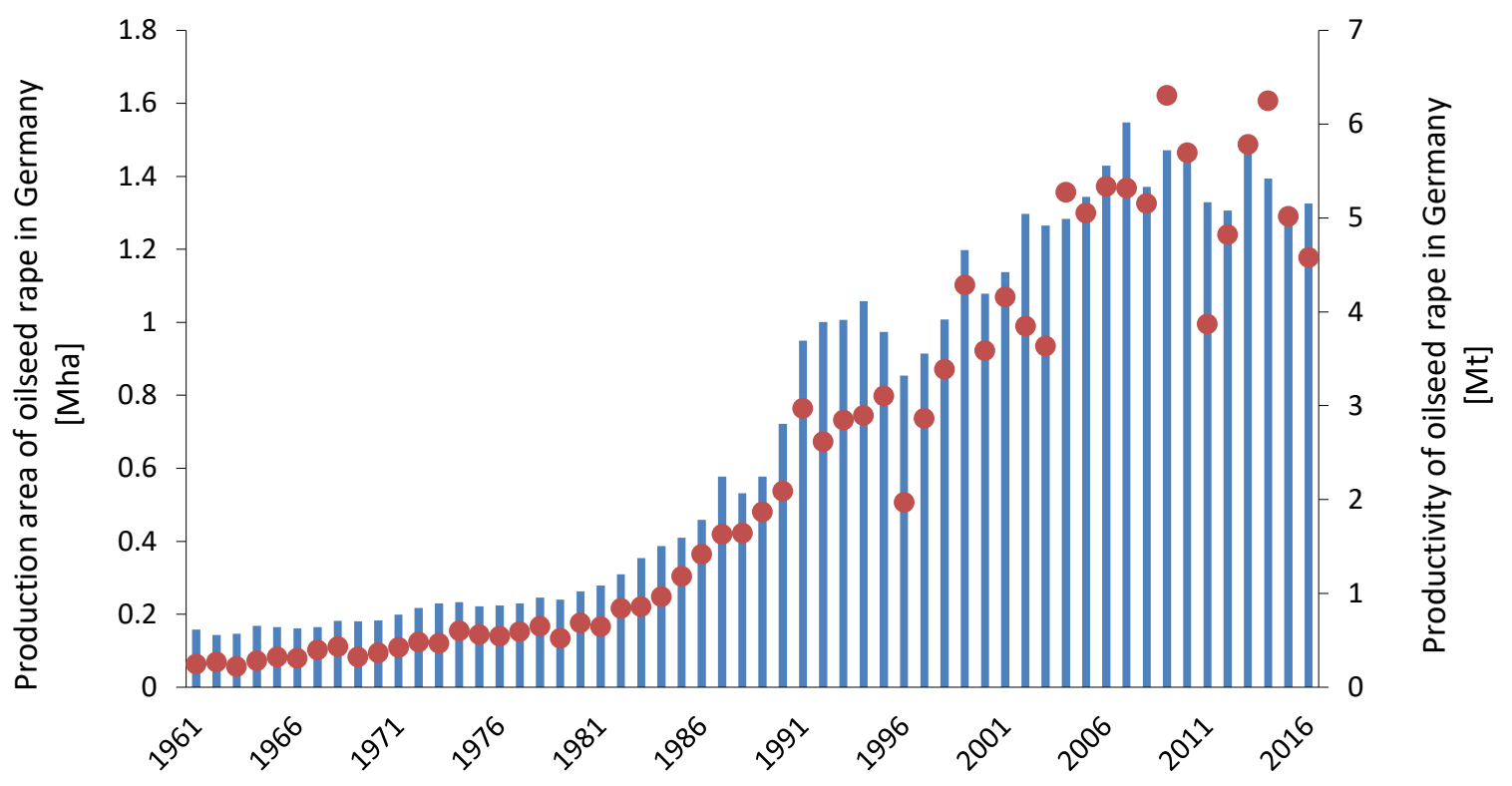

Fig. 1 Development of production area (blue) and productivity (red) of oilseed rape in Germany from 1961 to 2016 (FAOSTAT, 2018).

After having overcome nutritive constraints and gaining a dietary value, rapeseed oil is now appreciated as high-quality edible oil. It only contains small amounts of saturated fatty acids. In addition, the 2:1-ratio of the two polyunsaturated fatty acids linoleic acid and alpha-linolenic acid (C18:2 omega-6 and C18:3 omega-3, respectively) is beneficial to human health (Bell et al., 2001; Lin et al., 2013). Additionally, residues of rapeseed oil production such as oilcake and post-extraction rapeseed meal can now be used in livestock nutrition, showing a very good quality in terms of protein supply. Although rapeseed oil has now reached high food product quality, it is still also used in the non-food sector as biofuel and engine oil.

Oilseed rape is not only beneficial for human or animal health. In cereal based crop rotations, the introduction of oilseed rape as another crop breaks cereal disease cycles. In Western Europe and Germany, OSR is sown in August. After seedlings emerge from the soil, they quickly produce a dense rosette of true leaves. In this way, the soil is covered by the plant's biomass for a long period reducing erosion and nutrient leaching. Another typical characteristic of OSR is its long tap root. Reaching deep into the soil, it is able to incorporate nutrients from profound soil levels. This tap root also allows an enhancement of soil structure and soil aeration. Hence, OSR is a very beneficial component in a crop rotation when planted once in 4 to 6 years. Included in a too narrow crop rotation, though, it suffers 
from fungal diseases whose durable structures survive many years in the soil where the inoculum is accumulated. The most prevalent diseases of OSR in Germany are phoma canker (Leptosphaeria maculans and L. biglobosa), clubroot (Plasmodiophora brassicae), powdery mildew (Erysiphe cruciferarum), Verticillium stem striping (Verticillium longisporum) and sclerotinia stem rot (SSR) caused by Sclerotinia sclerotiorum (Lib.) de Bary (Christen \& Friedt, 2007).

\subsection{Sclerotinia sclerotiorum: life cycle and consequences of infection}

Sclerotinia sclerotiorum is a necrotrophic ascomycete and the causal agent of white mold and stem rot disease. Being present worldwide this devastating pathogen is able to infect more than 400 plant species, mostly dicotyledons (Boland \& Hall, 1994; Bolton et al., 2006). Besides crops like potato and sunflower, vegetable plants like egg plants, carrots, lettuce and also legumes like soy beans and beans, oilseed rape is infected by S. sclerotiorum, leading to sclerotinia stem rot (Adams \& Ayers, 1979; Boland \& Hall, 1994).

Sclerotinia sclerotiorum (Lib.) de Bary is assigned to the kingdom of Fungi, phylum Ascomycota, class Discomycetes, order Helotiales, family Sclerotiniaceae (Bolton et al., 2006; Derbyshire \& Denton-Giles, 2016). The formation of melanised hyphal aggregates, sclerotia, that represent the resting structures is a feature attributed to all members of the Sclerotiniaceae. Between two growing seasons, sclerotia are the means of fungal survival and source of new infections. After harvest, they remain in infested oilseed rape stubbles and are incorporated into the soil during tillage. Representing very tough survival structures barely affected by soil characteristics like temperature, moisture or $\mathrm{pH}$, sclerotia remain viable in the soil during more than five and up to ten years (Adams \& Ayers, 1979; Khangura et al., 2018). Given favourable environmental conditions, infection from sclerotia occurs by either myceliogenic or carpogenic germination. In the first, mycelium germinates from sclerotia and infects the below ground plant parts. This is the common infection process e.g. in sunflowers. Yet in oilseed rape, only infection following carpogenic germination has been reported with certainty (Abawi \& Grogan, 1979; Bolton et al., 2006). Apothecia stipes reach up to $3 \mathrm{~cm}$ in length, so only sclerotia not being buried too deep in the soil but remaining in the top 2 to $3 \mathrm{~cm}$ are functional (Abawi \& Grogan, 1979; Wu \& Subbarao, 2008). In spring, when soil moisture is appropriately high, and temperatures 
reach a level of 11 to $15^{\circ} \mathrm{C}$ (Abawi \& Grogan, 1979; Koch, 2005), carpogenic germination of sclerotia produces apothecia, the fruiting bodies of S. sclerotiorum. They emerge on the soil surface and expel their ascospores simultaneously (Bolton et al., 2006). After they landed on the plant surface and the presence of moisture presupposed, ascospores germinate. In OSR it is assumed that senescent petals serve as primary nutrition source to initiate ascospore germination (Abawi \& Grogan, 1979; Lumsden, 1979; Jamaux et al., 1995; Garg et al., 2010b), yet they do not seem to be essential because fully petalled and apetalous cultivars are both infected (Young \& Werner, 2012). However, petals bearing ascospores clearly facilitate infection: falling from the inflorescence, they land in leaf axils from where the infection by S. sclerotiorum starts and spreads (Fig. 2).

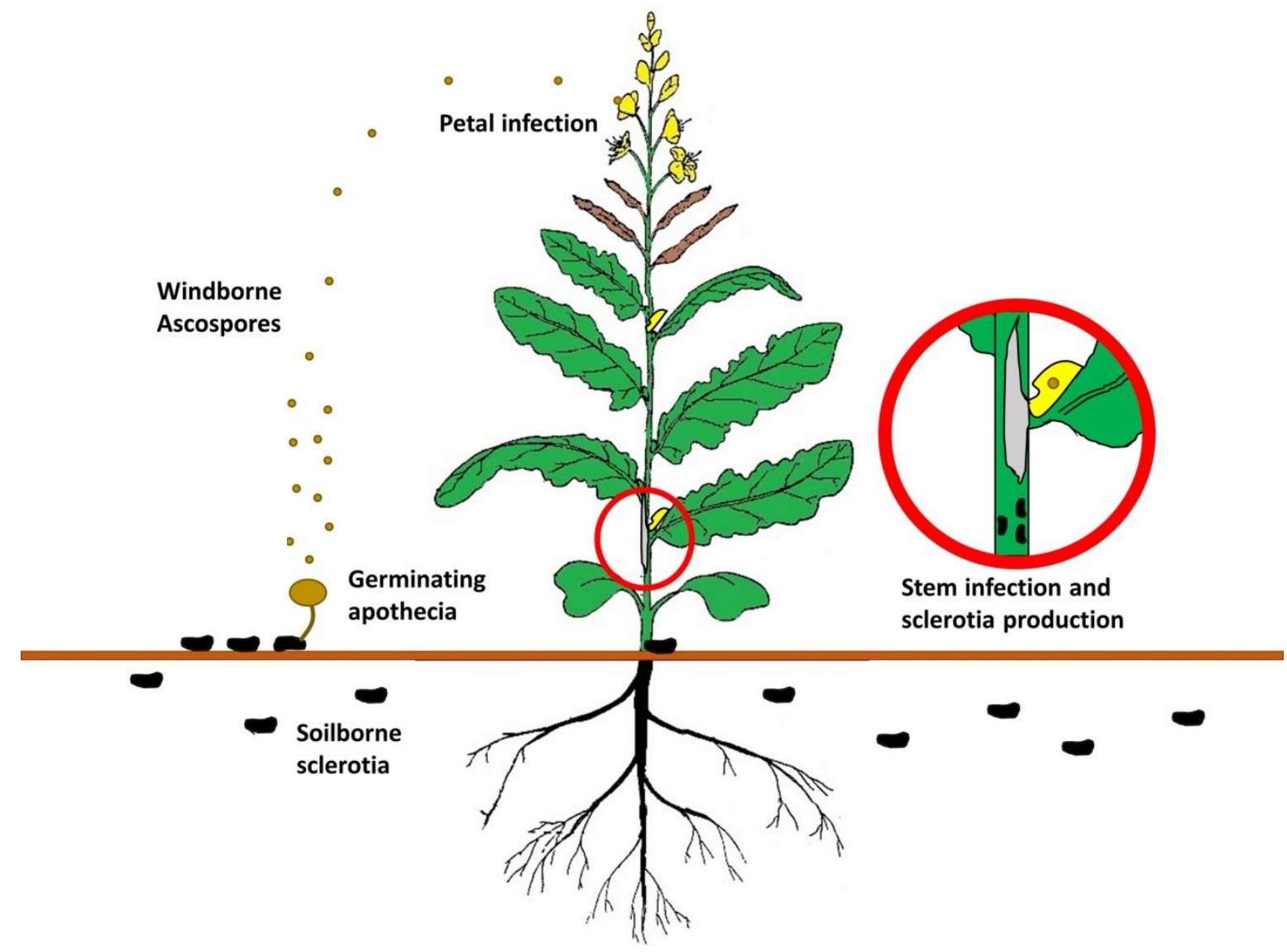

Fig. 2 Infection cycle of Sclerotinia sclerotiorum. Germinating apothecia release ascospores which are wind-dispersed to aboveground plant parts. Ascospores germinate and primarily infect senescing petal tissue. Infected petals abscise and lodge in leaf axils allowing the pathogen to penetrate and infect stems resulting in disease development. Following infection, sclerotia are formed, able to persist in the soil for several years (after Derbyshire \& Denton-Giles, 2016). 
Before penetrating the cell wall, hyphae form a simple appressorium, especially being observed in sensitive plant tissues like petals and leaves (Tariq \& Jeffries, 1986; Garg et al., 2010b; Davidson et al., 2016). However, it was widely assumed and finally confirmed that S. sclerotiorum also forms hyphal infection cushions for penetration of plant tissue (Jamaux et al., 1995; Davidson et al., 2016; Seifbarghi et al., 2017). Compared to the leaf surface, the stem might be more difficult to infect which may explain why penetration of the fungus needs more physical strength gained by an infection cushion (Hegedus \& Rimmer, 2005). As soon as the fungus has successfully reached the apoplast, hyphae are widening in girth and spread by intercellular growth (Kabbage et al., 2015; Davidson et al., 2016).

In this process, oxalic acid is considered as the most important pathogenicity factor of S. sclerotiorum (reviewed in Hegedus \& Rimmer, 2005). Although this compound secreted by the fungus is not considered essential for fungal pathogenicity (Xu et al., 2015; Davidson et al., 2016), it undoubtedly is a very powerful and multifaceted tool enhancing infection. The secretion of oxalic acid lowers the medium $\mathrm{pH}$ to establish optimal growth conditions for the fungus and hence assures its pathogenicity. Furthermore, it leads to the suppression of host oxidative burst, deregulates the stomatal guard cells, alters the host cellular redox status and, last but not least, leads to a better activity of cell wall degrading enzymes (CWDEs) by causing a decrease of extracellular pH to around 4-5 (Cessna et al., 2000; Guimarães \& Stotz, 2004; Bolton et al., 2006; Kim et al., 2008; Williams et al., 2011; Dickman \& Fluhr, 2013; Kabbage et al., 2013; Davidson et al., 2016). Besides, those processes are not all independent from each other. The $\mathrm{pH}$-reduction in combination with chelation of $\mathrm{Ca}^{2+}$ are the traits activating the CWDEs then being responsible for releasing nutrients required for the fungal growth. Accordingly, it has been proven recently that release of oxalic acid also directly enhances virulence of the pathogen (Xu et al., 2015). The initial $\mathrm{pH}$ of 5.5 being lowered to values between 3 and 4 in fact is the most important factor for pathogenicity. Hence, the pathogen is able to colonise the plant as described.

S. sclerotiorum is a necrotrophic pathogen (Hegedus \& Rimmer, 2005; Bolton et al., 2006; Garg et al., 2010b). The studies show a rapid cell death following the infection by the pathogen, whose growth is sustained by nutrients gained from necrotic host tissue. However, there is evidence that the pathogen is undergoing a brief biotrophic phase before switching to a necrotrophic lifestyle (Kabbage et al., 2015; Seifbarghi et al., 2017). This is 
probably the case in all necrotrophic pathogens, bearing in mind that first of all the fungus has to penetrate healthy tissue before it can develop within necrotic tissue of the host plant. This is supported by the finding that secretion of oxalate allows the pathogen to first suppress defence reactions of the plant and then establish infection, an attribute associated with hemi-biotrophic growth. Kabbage et al. (2015) explain that after the pathogen successfully suppressed defence responses of the host, transition to necrotrophy takes place, triggered by the increasing pressure of host defence.

Since $S$. sclerotiorum displays a broad host range, it can be assumed that it does not possess specifically adapted defence suppression mechanisms and thus is only remaining in the biotrophic phase until the first plant cell is invaded (Fig. 3). This hypothesis is supported by Davidson et al. (2016) who consider the infection of green tissue by S. sclerotiorum as two phases, (1) formation of a primary lesion and (2) colonisation of host tissue along with increasing concentrations of oxalic acid. Transcriptome analyses support the idea of the lifestyle transition. Seifbarghi et al. (2017) observed biotrophy-related effector genes in the first infection steps. An up-regulation of genes activating cuticle penetration occurred in the first hour post inoculation (hpi). In the following hours, the pathogen seems to react to plant defence responses. Meanwhile, genes encoding for oxidoreductase and hydrolase activity only were up-regulated until $24 \mathrm{hpi}$ and then declined introducing the necrotrophic phase which was acknowledged at $48 \mathrm{hpi}$ when necrotic lesions became visible. These findings show that each phase can be identified by unique expression profiles. Nevertheless, the first infections steps displaying biotrophic features should only be considered as a very brief transitional phase. In general, gene expression related to plant defence reactions such as anti-fungal activity - happens as an early defence response in the first 24 hours after inoculation (Zhao et al., 2009; Joshi et al., 2016) before the pathogen is taking over control completely. In the following infection stages, production of reactive oxygen species leads to programmed cell death (Kabbage et al., 2015; Seifbarghi et al., 2017). 


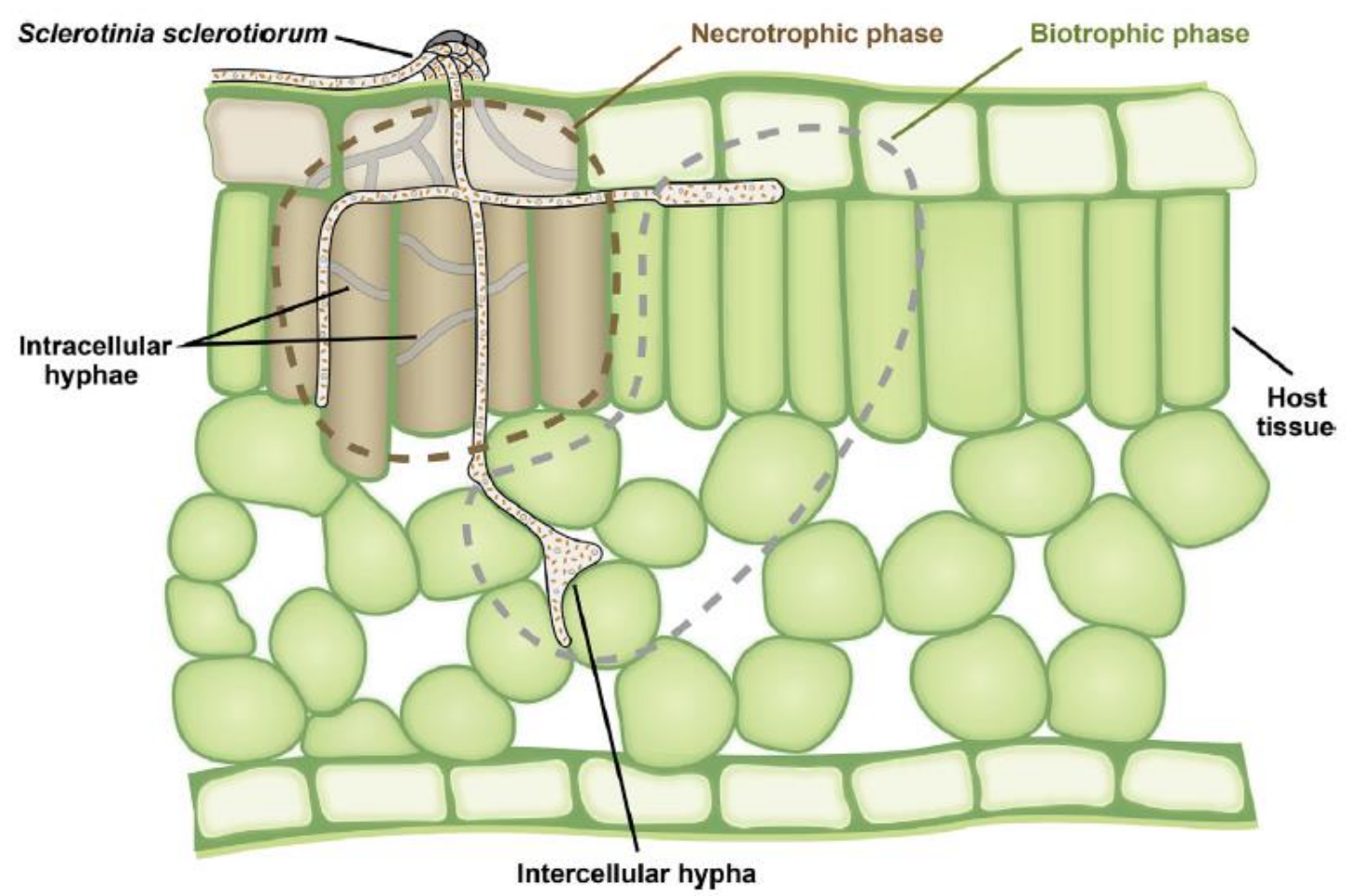

Fig. 3 During the early stage of infection S. sclerotiorum grows within the apoplastic space without crossing the plant cell wall. Secreting oxalic acid and other pathogenicity factors that modulate host cell defence responses, the fungus quickly switches to necrotrophic growth leaving a trail of dead cells, while biotrophy is restricted to the leading edge of fungal colonization (after Kabbage et al., 2015).

Another indication for transition is that penetration hyphae are thickening as soon as they invade the plant's tissue. This can be referred to the biotrophic phase of hemi-biotrophic fungi (Tariq \& Jeffries, 1984; Kabbage et al., 2015; Davidson et al., 2016). Furthermore, the low amount of cell wall degrading enzymes is supposed to play a role only after a successful infection to acquire nutrients (Lumsden \& Dow, 1973; Kabbage et al., 2015). These findings have been reported from leaves (Jamaux et al., 1995; Garg et al., 2010b; Kabbage et al., 2015; Davidson et al., 2016; Seifbarghi et al., 2017) but it remains unanswered whether the same strategy occurs in the stem, the actual site of disease development in oilseed rape.

After S. sclerotiorum has penetrated the epidermis of a plant, a clear and homogeneous symptom development similar in the different host species appears. Initially, water-soaked lesions become visible developing into necrotic, macerated tissue (Abawi \& Grogan, 1979; Bolton et al., 2006). On the plant surface, fluffy white mycelium is growing as only sign of infection. However, when the fungus advances into the main stem, wilting symptoms appear downstream the lesion. With the spreading disease, aged lesions appear bleached and shrunk. Typically, sclerotia are formed inside the infected tissue without any evidence 
of external mycelium. Nevertheless, the disease can spread within the field via plant-toplant contact. In the end, sclerotia form in the pith of the stem as the inoculum for the next growing season.

Only few studies exist investigating yield losses due to infection by Sclerotinia sclerotiorum. Estimating damage and connecting it to disease incidence is a complex task due to the variability. Over all, an average disease incidence of $10-20 \%$ is associated with yield losses of 5-10\% (del Río et al., 2007). Moreover, Liu (2004) reported yield loss of 30\% due to an early break of siliques followed by a reduced seed amount and lower thousand seed weight. In Germany, up to $0.40 \%$ yield loss may occur per percent disease incidence (Dunker, 2005), whereas in Australia losses even up to 1.3\% have been reported (Kirkegaard et al., 2006). In cases of high disease incidence, yield losses can reach up to $4 \mathrm{dt} / \mathrm{ha}$ (Dunker, 2005). No distinct effect on thousand seed weight or oil quality is visible. Economic efficiency of fungicide application is variable. It always depends on whether the fungicide application at flowering time was really affecting infection and disease development. To facilitate application decisions, forecasting systems, such as ScleroPro, are available but they are not always helpful to manage the disease (Karl Fricke, personal communication, August 2016). Furthermore, fungicide application is only economically effective when high disease pressure occurs. This, however, has been rarely reported during the past 20 years even in intense cultivation areas like Schleswig-Holstein, Germany, where severe infections only occurred in 1998, 2001, 2004 and 2016 (results of field testing for licensing procedure for new varieties (Wertprüfung), Bundessortenamt, Germany, Dr. Richard Manthey, personal communications 2017).

Under low disease pressure, oilseed rape plants are able to compensate damaging effects of the infection on yield (del Río et al., 2007). Nevertheless, sclerotinia stem rot (SSR) can not only cause yield losses, but it also constitutes a consistently recurring disease due to an accumulation of inoculum in the soil. Therefore, precise and effective management is necessary. As preventive measure, ploughing is helpful because sclerotia are buried in deeper soil layers. Hence, apothecia cannot reach the soil surface and sclerotia lose their functionality (Abawi \& Grogan, 1979). However, in view of the fact that only a narrow crop rotation is the standard system widely distributed, ploughing might bring previously buried sclerotia back to the soil surface negating its positive effect. Therefore, a wide crop rotation 
including non-host plants may reduce disease pressure and is commonly considered as the key control component for SSR (Derbyshire \& Denton-Giles, 2016). The management strategy of a wide crop rotation still needs to be complemented with the disruption of the live cycle of the pathogen. In this regard, regulation of numerous weeds serving as hosts and thus as green bridge is indispensable (Adams \& Ayers, 1979; Boland \& Hall, 1994).

Attempts to adapt biological agents to practical use were made with Bacillus subtilis (Hu et al., 2014) and Trichoderma harzianum (Troian et al., 2014) and were successful in case of Coniothyrium minitans (Daniel Karsch, Bayer CropScience Biologics $\mathrm{GmbH}$, personal communications, $6^{\text {th }}$ July 2016). Yet the primary method to control SSR remains direct, chemical crop protection by synthetic fungicide application (Bradley et al., 2006b; Derbyshire \& Denton-Giles, 2016). Due to its monocyclic infection cycle, S. sclerotiorum holds only a small risk for developing fungicide resistance. Nevertheless, fungicide treatment holds a couple of disadvantages. First, the optimal application window is quite small depending on environmental factors having an impact on ascospore distribution and successful plant infection. As described above, forecasting systems provide decision support but since it is still difficult to predict disease outbreak, optimal timing remains somehow ambiguous. Second, given that sclerotinia stem rot is considered as a sporadic disease (Kirkegaard et al., 2006), prophylactic treatments are often non-profitable and even a reduction of disease incidence after spraying does not necessarily enhance the yield (Dunker, 2005; Bradley et al., 2006b; del Río et al., 2007). Third, non-target organisms like pollinators and beneficial insects might be impaired, thus, only application is restricted to certain products and day times. Farmers are obliged to spray in late evening hours when less honey bees are flying. Furthermore, the new technique of dropleg application enables a more targeted and equally efficient application on the plant stem (Dr. Dominik Dicke, Pflanzenschutzdienst Gießen, personal communications, $14^{\text {th }}$ February 2017).

\subsection{Quantitative disease resistance in B. napus against S. sclerotiorum}

Regarding the fact that current disease management of sclerotinia stem rot is not sustainable and considering the growing standard for environmental protection leading to the ban of pesticides, more future oriented disease management is necessary. For this reason, breeding of resistant genotypes is crucial, especially because among current 
cultivars, genotypes possessing effective resistance against S. sclerotiorum are not available until today (Liu, 2004; Bradley et al., 2006a; Derbyshire \& Denton-Giles, 2016). For designing proper breeding strategies, it is important to characterise and understand defence mechanisms leading to enhanced resistance in OSR cultivars against S. sclerotiorum.

It is known since long time that disease resistance can occur in two ways: (1) complete, monogenic qualitative resistance and (2) incomplete, polygenic quantitative resistance. Poland et al. (2009) described quantitative disease resistance (QDR) as a reduction, not the absence of disease. QDR and the underlying mechanisms are difficult to understand. Many loci may contribute to resistance and hence the resistance phenotype is only displayed in a very inconsistent way (St Clair, 2010; French et al., 2016; Corwin \& Kliebenstein, 2017). In contrast to qualitative resistance conditioned by highly effective R-genes, quantitative resistance is more durable and provides defence against a wide range of pathogens. Furthermore, while R-genes mediate a strong defence response - especially against biotrophic pathogens - the following hypersensitive reaction would only increase the susceptibility against necrotrophic pathogens (Govrin \& Levine, 2000; Glazebrook, 2005; Jones \& Dangl, 2006; Poland et al., 2009). In contrast to that, quantitative resistance is more complex. Quantitative defence mechanisms triggered by pathogenic attack usually are linked to signalling pathways (Corwin \& Kliebenstein, 2017). Downstream resistance mechanisms are activated, such as the production of defence hormones like ethylene or salicylic acid, or secondary metabolites, like phenolic compounds and glucosinolates, revealing a broad range of manifold tools (Zhao et al., 2007; Wei et al., 2015; Corwin \& Kliebenstein, 2017). Phenolics, peroxidase and glucosinolates are described in the following part of QDR.

\section{Phenolic acids}

Phenolic acids are low molecular weight secondary metabolites being part of the phenylpropanoid pathway and involved in initial defence responses (Nicholson \& Hammerschmidt, 1992; Dixon et al., 2002). They can have direct effects on pathogenic organisms. As precursors of lignin, they have indirect effects by strengthening of the cell wall as mechanical barrier and are hence inhibiting fungal colonisation (Malinovsky et al., 2014; Miedes et al., 2014; Kumar et al., 2016). Phenylalanine ammonia lyase (PAL) is the 
primary enzyme involved in the phenylpropanoid pathway. Furthermore, it is regarded as an important defence enzyme because it is responsible for catalysing the elimination of ammonia from phenylalanine to $t$-cinnamic acid (Dixon \& Paiva, 1995; Wang et al., 2004; Zhao et al., 2007). In addition, PAL is reported to be up-regulated under infection (Dixon et al., 2002; Oliveira et al., 2015). In a study on Camelina sativa it has been shown that monolignol synthesis is up-regulated after infection with S. sclerotiorum (Eynck et al., 2012). In this plant species, phenolics accumulated only in the resistant genotype near the site of pathogen ingress. Furthermore, transcript genes regulating early steps in the phenyIpropanoid pathway (e.g. PAL) were down-regulated, especially in the susceptible line. Similar findings were reported in a recent study on oilseed rape (Höch, 2016). Consequently, early synthesised phenolic acids should be quantified (Fig. 4). Among them, $t$-cinnamic acid represents a switch between salicylic acid synthesis, known to have important functions in defence against vascular pathogens (Eynck et al., 2007; Ratzinger et al., 2009; Zheng, 2018) and phenolic acids being precursors of monolignols polymerised to lignin. These precursors, such as p-coumaric acid, chlorogenic acid, caffeic acid, ferulic acid and sinapic acid are therefore important parts of plant defence mechanisms. 


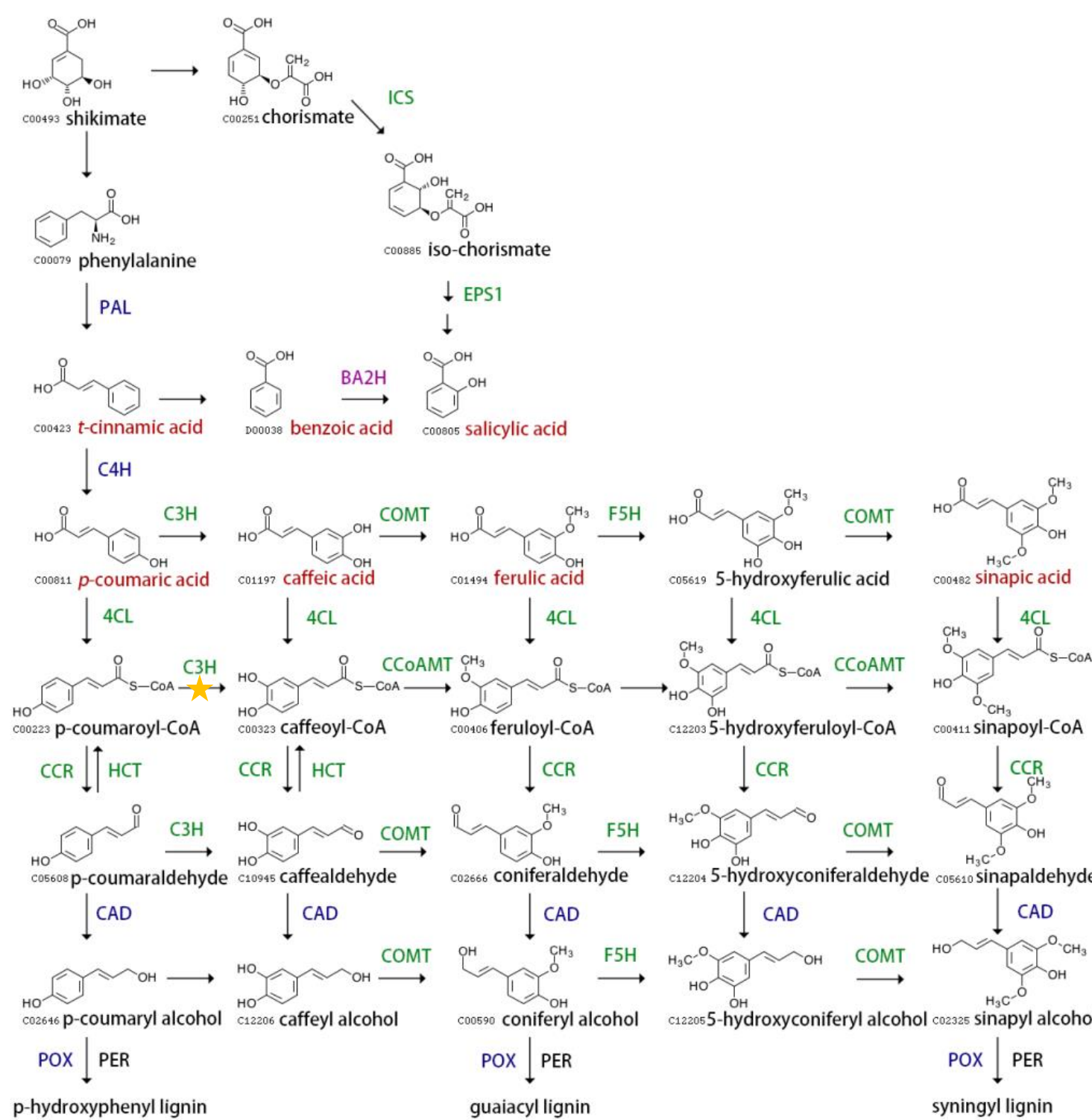

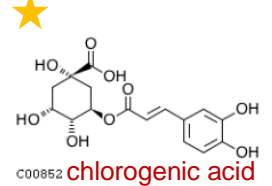

Fig. 4 Phenylpropanoid pathway with the primary synthesised phenolic acids marked in red (after KEGG bna0049; Dixon \& Paiva, 1995; Fraser \& Chapple, 2011; Zhao et al., 2013; Alvarez et al. 2016). 


\section{Peroxidase activity}

Class III plant peroxidases (POX) are glycoproteins located in vacuoles and cell walls (Passardi et al., 2005). POX is needed to cleave hydrogen peroxide $\left(\mathrm{H}_{2} \mathrm{O}_{2}\right)$ occurring in the plant tissue because of the so-called oxidative burst, the accumulation of reactive oxygen species such as $\mathrm{H}_{2} \mathrm{O}_{2}$. The consequential programmed cell death is a very effective plant defence response against biotrophic pathogens preventing living tissue from serving as nutritional source for the fungus (Reuveni et al., 1992; Lamb \& Dixon, 1997). To prevent further cell damage, $\mathrm{POX}$ catalyses the reduction from $\mathrm{H}_{2} \mathrm{O}_{2}$ to water and thereby detoxifies the reactive oxygen species (Asada, 1992; von Tiedemann, 1997; Zhao et al., 2005). In a biotrophic pathosystem, POX activity is associated with resistance (Reuveni et al., 1992). In a necrotrophic relationship, $\mathrm{H}_{2} \mathrm{O}_{2}$ is merely produced by the pathogen with the objective of killing host cells and transform them into an energy source (Govrin \& Levine, 2000; Almagro et al., 2009; Horbach et al., 2011, and references therein), so by infection, fungal necrotrophs trigger cell death and promote their proliferation (Kim et al., 2008). At the same time, there is always a basal level of POX activity in plants to ensure housekeeping functions such as participation in the biosynthesis of lignin as constitutive cell wall component (Fig. 4; Ros Barceló et al., 2007; O’Brien et al., 2012). The enigma of reactive oxygen species in plant-pathogen interactions is that they are required for establishment of the pathogen and also for resistance of the host (Foley et al., 2016). Therefore, POX represents an interesting link between stress reactions and defence mechanisms.

\section{Glucosinolates}

Glucosinolates (GSL) are $\beta$-thioglucoside- $N$-hydroxysulfates with a side chain and a sulfurlinked $\beta$-D-glucopyranose moiety. They are almost exclusively synthesised by the order Capparales including Brassicaceae and occur as inactive precursors of breakdown products with antimicrobial activity (Fahey et al., 2001). These breakdown products also own antinutritional properties, which is why seed GSL content has been successfully decreased by breeding. GSL are produced in the main plant tissues, such as leaves, where high concentrations can be found. Long distance transport from maternal tissue to the embryo follows the phloem, probably as intact GSL (Mithen, 1992; Chen et al., 2001). GSL are synthesised from different amino acids and therefore are represented as three different classes. The most abundant ones are aliphatic GSL with mainly methionine as precursor. 
Aromatic GSL derive from phenylalanine and are less dominant. Indole GSL are based on tryptophan. Those are mainly present in the stem and are known to react to environmental stresses (Mithen, 1992; Bartlet et al., 1999; Halkier \& Gershenzon, 2006). Quantitative trait loci (QTL) responsible for the different classes are not correlated, hinting on independent regulation (Li et al., 1999a; Kliebenstein et al., 2001) and therefore diverse distribution among plant tissues as well as different reaction to infection.

\subsection{Aim of study}

Oilseed rape varieties cultivated in Europe show a high susceptibility to the devastating pathogen Sclerotinia sclerotiorum. Previous studies have shown that at presence resistant cultivars are lacking (Wulf, 2011; Höch, 2016). To complete the picture, a new set of different Brassica napus genotypes was assessed for disease response in order to find promising sources of resistance in the Brassica gene pool for future breeding strategies.

Two varieties with contrasting responses to infection were selected for in-depth studies to identify the physiological and histological mechanisms making a plant more resistant to S. sclerotiorum. All experiments focussed on the difference between (1) a susceptible and partially resistant genotype and (2) different plant tissues, namely stem, hypocotyl and root. In former studies, comparison of plant tissues has been shown to be more pronounced than genotypic differences. The evaluation of tissue specific responses aimed at answering the question whether resistance sources might rely on tissue specific responses to infection and whether traits specific to a plant tissue could be identified as general resistance factors. Defence responses such as glucosinolate accumulation or shift in the pattern of phenolic compounds, and changes of peroxidase activity after inoculation with S. sclerotiorum were studied to elucidate whether they play a role in quantitative disease resistance. Furthermore, the colonisation strategy of fungal hyphae in the plant tissue was observed. Overall, the objective was to characterise genotypic and tissue specific resistance in oilseed rape (B. napus) against Sclerotinia sclerotiorum and to shed light into the underlying mechanisms. 


\section{Materials and methods}

\subsection{Chemicals and other materials}

The different materials and substances used during the varying experiments within this work are listed below (Tab. 1 and Tab. 2).

Tab. 1 Utilised chemicals and their manufacturer.

\begin{tabular}{|c|c|}
\hline Chemicals & Manufacturer \\
\hline Agar-agar & Carl Roth GmbH \& Co. KG, Karlsruhe, GER \\
\hline Aniline blue & Sigma-Aldrich Chemie GmbH, Steinheim, GER \\
\hline Borax, fused $\left(\mathrm{B}_{4} \mathrm{Na}_{2} \mathrm{O}_{7}\right)$ & Sigma-Aldrich Chemie GmbH, Steinheim, GER \\
\hline Calcium nitrate $\left(\mathrm{Ca}\left(\mathrm{NO}_{3}\right)_{2}\right)$ & Merck KGaA, Darmstadt, GER \\
\hline Cetyltrimethylammonium bromide (CTAB) & Carl Roth GmbH \& Co. KG, Karlsruhe, GER \\
\hline Chlorazol black E (CBE) & Sigma-Aldrich Chemie GmbH, Steinheim, GER \\
\hline Chloroform & Carl Roth GmbH \& Co. KG, Karlsruhe, GER \\
\hline Cobald chloride $\left(\mathrm{CoCl}_{2} \bullet 6 \mathrm{H}_{2} \mathrm{O}\right)$ & Merck KGaA, Darmstadt, GER \\
\hline Copper sulfate $\left(\mathrm{CuSO}_{4} \bullet 5 \mathrm{H}_{2} \mathrm{O}\right)$ & Carl Roth GmbH \& Co. KG, Karlsruhe, GER \\
\hline 2,6-di-tert-butyl-4-methylphenol (DBC) & Sigma-Aldrich Chemie GmbH, Steinheim, GER \\
\hline Disodium phosphate $\left(\mathrm{Na}_{2} \mathrm{HPO}_{4}\right)$ & AppliChem GmbH, Darmstadt, GER \\
\hline Ethanol & Carl Roth GmbH \& Co. KG, Karlsruhe, GER \\
\hline Ethidium bromide & AppliChem GmbH, Darmstadt, GER \\
\hline Ethyl acetate & Carl Roth GmbH \& Co. KG, Karlsruhe, GER \\
\hline Ethylenediaminetetraacetic acid (EDTA) & AppliChem GmbH, Darmstadt, GER \\
\hline Formaldehyde solution & Carl Roth GmbH \& Co. KG, Karlsruhe, GER \\
\hline Glacial acetic acid & Carl Roth GmbH \& Co. KG, Karlsruhe, GER \\
\hline Glycerol & Carl Roth GmbH \& Co. KG, Karlsruhe, GER \\
\hline \multicolumn{2}{|l|}{ Growth regulators } \\
\hline Caramba (metconazole) & BASF SE, Limburgerhof, GER \\
\hline Carax (mepiquat chloride + metconazole) & BASF SE, Limburgerhof, GER \\
\hline Moddus (trinexapac-ethyl) & Syngenta Agro GmbH, Maintal, GER \\
\hline Toprex (paclobutrazol + difenoconazole) & Syngenta Agro GmbH, Maintal, GER \\
\hline
\end{tabular}


Guaiacol

Hydrochloric acid $(\mathrm{HCl})$

Hydrogen peroxide $\left(\mathrm{H}_{2} \mathrm{O}_{2}\right)$

Iron sulfate $\left(\mathrm{FeSO}_{4} \bullet 7 \mathrm{H}_{2} \mathrm{O}\right)$

Isomyl alcohol

Lactic acid

Magnesium sulfate $\left(\mathrm{MgSO}_{4} \bullet 7 \mathrm{H}_{2} \mathrm{O}\right)$

Manganese chloride $\left(\mathrm{MnCl}_{2} \bullet 4 \mathrm{H}_{2} \mathrm{O}\right)$

Methanol

Monopotassium phosphate $\left(\mathrm{KH}_{2} \mathrm{PO}_{4}\right)$

Monosodium phosphate $\left(\mathrm{NaH}_{2} \mathrm{PO}_{4}\right)$

Perboric acid $\left(\mathrm{BHO}_{3}\right)$

Periodic acid

Pianese stain solution

Polyvinylpyrrolidone K90

Potassium chloride $(\mathrm{KCl})$

Potassium disulfite $\left(\mathrm{K}_{2} \mathrm{~S}_{2} \mathrm{O}_{5}\right)$

Potassium hydroxide ( $\mathrm{KOH})$

Potassium nitrate $\left(\mathrm{KNO}_{3}\right)$

Potato Dextrose Agar (PDA)

Potato Dextrose Broth (PDB)

Propidium iodide

Proteinase $\mathrm{K}$

Roti $^{\circledR}$-Histol

Schiff's reagent

Silver nitrate $\left(\mathrm{AgNO}_{3}\right)$

Sodium acetate $\left(\mathrm{CH}_{3} \mathrm{COONa}\right)$

Sodium chloride $(\mathrm{NaCl})$

Sodium hydroxide $(\mathrm{NaOH})$

Sodium molybdate $\left(\mathrm{Na}_{2} \mathrm{MoO}_{4} \cdot 2 \mathrm{H}_{2} \mathrm{O}\right)$

Toluidine blue $\mathrm{O}$
Sigma-Aldrich Chemie GmbH, Steinheim, GER

Carl Roth GmbH \& Co. KG, Karlsruhe, GER

Merck KGaA, Darmstadt, GER

Merck KGaA, Darmstadt, GER

AppliChem GmbH, Darmstadt, GER

Merck KGaA, Darmstadt, GER

AppliChem GmbH, Darmstadt, GER

Sigma-Aldrich Chemie $\mathrm{GmbH}$, Steinheim, GER

Carl Roth GmbH \& Co. KG, Karlsruhe, GER

Merck KGaA, Darmstadt, GER

Carl Roth GmbH \& Co. KG, Karlsruhe, GER

Carl Roth GmbH \& Co. KG, Karlsruhe, GER

Sigma-Aldrich Chemie GmbH, Steinheim, GER

Morphisto $\mathrm{GmbH}$, Frankfurt am Main, GER

AppliChem GmbH, Darmstadt, GER

Merck KGaA, Darmstadt, GER

Merck KGaA, Darmstadt, GER

Carl Roth GmbH \& Co. KG, Karlsruhe, GER

Carl Roth GmbH \& Co. KG, Karlsruhe, GER

Sigma-Aldrich Chemie GmbH, Steinheim, GER

Carl Roth GmbH \& Co. KG, Karlsruhe, GER

Carl Roth GmbH \& Co. KG, Karlsruhe, GER

AppliChem GmbH, Darmstadt, GER

Carl Roth GmbH \& Co. KG, Karlsruhe, GER

Carl Roth GmbH \& Co. KG, Karlsruhe, GER

Carl Roth GmbH \& Co. KG, Karlsruhe, GER

Carl Roth GmbH \& Co. KG, Karlsruhe, GER

Carl Roth GmbH \& Co. KG, Karlsruhe, GER

Merck KGaA, Darmstadt, GER

Merck KGaA, Darmstadt, GER

Merck KGaA, Darmstadt, GER 
Trypan blue

Wheat germ agglutinin,

Alexa Fluor ${ }^{\mathrm{TM}} 488$ conjugate

Zinc sulfate $\left(\mathrm{ZnSO}_{4} \bullet 7 \mathrm{H}_{2} \mathrm{O}\right)$
AppliChem GmbH, Darmstadt, GER

Thermo Fisher Scientific, Waltham,

Massachusetts, USA

Merck KGaA, Darmstadt, GER

Tab. 2 Utilised materials.

\begin{tabular}{|c|c|}
\hline Material & Manufacturer \\
\hline 96-well flat bottom assay plate & Costar incorporated, Kennebunk, Maine, USA \\
\hline 384-well plates & Kisker Biotech GmbH \& Co. KG, Steinfurt, GER \\
\hline Centrifuge tube (15 and $50 \mathrm{ml}$ ) & Sarstedt AG \& Co., Nümbrecht, GER \\
\hline Cover slides (24 × 60 mm; No 1.5) & $\begin{array}{l}\text { Paul Marienfeld GmbH \& Co. KG, Lauda- } \\
\text { Königshofen, GER }\end{array}$ \\
\hline HPLC vials N9 & Macherey-Nagel, Düren, GER \\
\hline Microtome blade MX35 Premier+ & $\begin{array}{l}\text { Thermo Fisher Scientific, Waltham, Massachusetts, } \\
\text { USA }\end{array}$ \\
\hline Paraffin Histoplast & $\begin{array}{l}\text { Thermo Fisher Scientific, Waltham, Massachusetts, } \\
\text { USA }\end{array}$ \\
\hline Parafilm $\mathrm{M}^{\circledR}$ & Bemis Company Inc., Wisconsin, USA \\
\hline Petri dishes & \\
\hline Round $(90 \times 16 \mathrm{~mm})$ & Sarstedt AG \& Co., Nümbrecht, GER \\
\hline Square $(100 \times 100 \times 20 \mathrm{~mm})$ & Sarstedt AG \& Co., Nümbrecht, GER \\
\hline qPCRBIO SyGreen Mix Lo-Rox & Nippon Genetics Europe GmbH, Düren, GER \\
\hline Reaction tubes $(0.2,1.5$ and $2 \mathrm{ml})$ & Sarstedt AG \& Co., Nümbrecht, GER \\
\hline Sefar Nitex 03-70/33 & Sefar AG, Thal, Switzerland \\
\hline Specimen slides SuperFrost ${ }^{\circledR}$ & Carl Roth GmbH \& Co. KG, Karlsruhe, GER \\
\hline
\end{tabular}




\subsection{Media and solutions}

Following media were used for fungal culture and in vitro studies:

$\underline{\text { Half concentrated potato dextrose agar (PDA) for S. sclerotiorum cultivation }}$

PDA $19.5 \mathrm{~g}$

Agar

$7.5 \mathrm{~g}$

$\mathrm{dH}_{2} \mathrm{O}$

$1000 \mathrm{ml}$

Autoclave at $121^{\circ} \mathrm{C}$ for 20 minutes.

PNM-medium

$\begin{array}{ll}\mathrm{KNO}_{3} & 5 \mathrm{mM} \\ \mathrm{MgSO}_{4} \cdot 7 \mathrm{H}_{2} \mathrm{O} & 2 \mathrm{mM} \\ \mathrm{Ca}\left(\mathrm{NO}_{3}\right)_{2} & 2 \mathrm{mM} \\ \text { Fe-EDTA } & 2.5 \mathrm{ml} / \text { litre } \\ \text { Micronutrient-mix } & 1.0 \mathrm{ml} / \text { litre } \\ \text { Agar } & 10.0 \mathrm{~g} / \text { litre }\end{array}$

Sterilise at $121^{\circ} \mathrm{C}$ for $20 \mathrm{~min}$ and adjust $\mathrm{pH}$ to 5.6 by adding $2.5 \mathrm{ml}$ filter sterilized $1 \mathrm{M}$ $\mathrm{KH}_{2} \mathrm{PO}_{4}$ under sterile conditions.

Fe-EDTA for PNM-medium

$\begin{array}{ll}\text { Sterile } \mathrm{H}_{2} \mathrm{O} & 400 \mathrm{ml} \\ \mathrm{FeSO}_{4} \cdot 7 \mathrm{H}_{2} \mathrm{O} & 2.5 \mathrm{~g} \\ \mathrm{Na}_{2}-\mathrm{EDTA} \cdot 2 \mathrm{H}_{2} \mathrm{O} & 3.6 \mathrm{~g}\end{array}$

Heat to boil in microwave, stir for $30 \mathrm{~min}$ while cooling and bring to a final volume of $450 \mathrm{ml}$.

Micronutrient-mix for PNM-medium

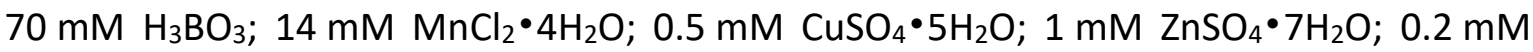
$\mathrm{Na}_{2} \mathrm{MoO}_{4} \cdot 2 \mathrm{H}_{2} \mathrm{O} ; 10 \mathrm{mM} \mathrm{NaCl} ; 0.01 \mathrm{mM} \mathrm{COCl}{ }_{2} \cdot 6 \mathrm{H}_{2} \mathrm{O}$ 
Following solutions and stains were used for histological studies:

Acetic acid-formaldehyde-ethanol solution (AFE)

Ethanol (96\%)

$90 \%$

Acetic acid (99\%)

$5 \%$

Formaldehyde solution (37\%)

$5 \%$

Phosphate buffered saline (PBS) $\mathrm{pH} 7.2$

$\begin{array}{ll}\mathrm{NaCl} & 140 \mathrm{mM} \\ \mathrm{Na}_{2} \mathrm{HPO}_{4} & 10 \mathrm{mM} \\ \mathrm{NaH}_{2} \mathrm{PO}_{4} & 3.3 \mathrm{mM} \\ \mathrm{KCl} & 2.7 \mathrm{mM}\end{array}$

Warm saturated Roti ${ }^{\circledR}$-Histol/Paraffin

Histoplast

$80 \mathrm{~g}$

Roti $^{\circledR}$-Histol

$120 \mathrm{ml}$

Chlorazol black $\mathrm{E}$

Chlorazol black E

$0.03 \mathrm{~g}$

$\mathrm{ddH}_{2} \mathrm{O}$

$33.3 \mathrm{ml}$

Lactic acid (80\%)

$33.3 \mathrm{ml}$

Alexa Fluor ${ }^{\mathrm{TM}} 488$ conjugate, $50 \mu \mathrm{g} / \mathrm{ml}$

Stock: Alexa Fluor

$5 \mathrm{mg}$

dd $\mathrm{H}_{2} \mathrm{O}$

$5 \mathrm{ml}$

Dye: Stock solution

$50 \mu \mathrm{l}$

$\mathrm{dd}_{2} \mathrm{O}$

$950 \mu \mathrm{l}$

Propidium iodide, $10 \mu \mathrm{g} / \mathrm{ml}$

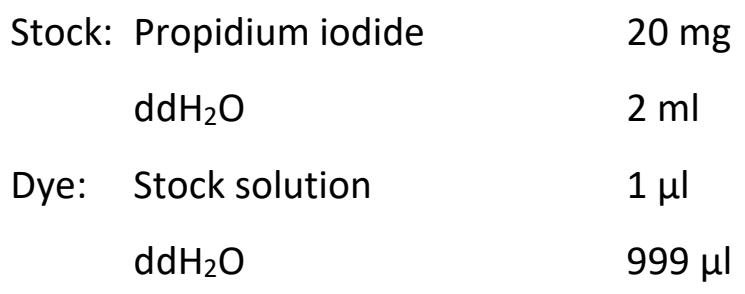


Toluidine blue $0,0.1 \%$

Toluidine blue $\mathrm{O}$

$0.1 \mathrm{~g}$

$1 \%$ Borax

$100 \mathrm{ml}$

Trypan-aniline blue double stain, $0.05 \%$

Prepare stains separately

\begin{tabular}{|c|c|c|c|}
\hline Stock: Trypan blue & $0.005 \mathrm{~g}$ & Aniline blue & $0.005 \mathrm{~g}$ \\
\hline $\mathrm{dd}_{2} \mathrm{O}$ & $1 \mathrm{ml}$ & $\mathrm{KH}_{2} \mathrm{PO}_{4}$ & $1 \mathrm{ml}$ \\
\hline Stock solution & $100 \mu \mathrm{l}$ & Stock solution & $100 \mu l$ \\
\hline $\mathrm{dd}_{2} \mathrm{O}$ & $900 \mu l$ & $\mathrm{KH}_{2} \mathrm{PO}_{4}$ & $900 \mu \mathrm{l}$ \\
\hline
\end{tabular}

Periodic acid Schiff's assay (PAS)

For oxidation before and differentiation after staining procedure

Oxidation reagent:

$\begin{array}{ll}\text { Periodic acid } & 800 \mathrm{mg} \\ \mathrm{dH}_{2} \mathrm{O} & 90 \mathrm{ml} \\ 0.2 \mathrm{M} \text { sodium acetate } & 10 \mathrm{ml}\end{array}$

$\mathrm{SO}_{2}$-water:

$10 \% \mathrm{~K}_{2} \mathrm{~S}_{2} \mathrm{O}_{5} \quad 10 \mathrm{ml}$

$1 \mathrm{~N} \mathrm{HCl} \quad 10 \mathrm{ml}$

$\mathrm{dH}_{2} \mathrm{O} \quad 180 \mathrm{ml}$ 
Following solutions were used for peroxidase activity essay:

Sodium phosphate buffer, $50 \mathrm{mM}$

$91.5 \mathrm{ml}$ of $2.98 \mathrm{mM} \mathrm{Na}_{2} \mathrm{HPO}_{4} \cdot 2 \mathrm{H}_{2} \mathrm{O}$ are adjusted to $\mathrm{pH} 7.9$ with $1.59 \mathrm{mM} \mathrm{NaH}_{2} \mathrm{PO}_{4} \cdot 2 \mathrm{H}_{2} \mathrm{O}$.

$50 \mathrm{mM}$ SPB ( $\mathrm{pH} 7.8$ ), and after that filled up to a volume of $400 \mathrm{ml}$ with water.

Sodium phosphate buffer, $25 \mathrm{mM}$

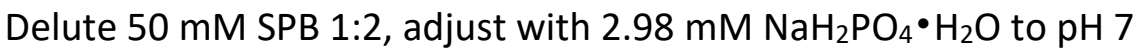

\section{Enzyme extraction solution}

Sample ground in liquid nitrogen $\quad 0.1 \mathrm{~g}$

$50 \mathrm{mM}$ SPB 1\% polyvinylpyrrolidone $2 \mathrm{ml}$

$\underline{\text { Reaction solution }}$

$\begin{array}{ll}\text { Guaiacol } & 22 \mu \mathrm{l}(\hat{=} 0.05 \% \text { in } 50 \mathrm{ml}) \\ 35 \% \mathrm{H}_{2} \mathrm{O}_{2} & 56.1 \mu \mathrm{l}(\hat{=} 10 \mathrm{mM} \text { in } 50 \mathrm{ml}) \\ 25 \mathrm{mM} \mathrm{SPB}(\mathrm{pH} \mathrm{7.0)} & 50 \mathrm{ml}\end{array}$




\subsection{Plant materials and cultivation for greenhouse experiments}

Screening of different genotypes of Brassicaceae was conducted to assess the range of resistance in a diversity set. In total, 199 accessions were chosen from the Brassica napus Diversity Fixed Foundation Set (BnaDFFS) including two reference oilseed rape (OSR) varieties from the University of Göttingen were screened for susceptibility or resistance against Sclerotinia sclerotiorum. BnaDFFS accessions have been propagated in 2015 by John Innes Centre, Norwich, England. All genotypes belonged to the same species, Brassica napus, but were of different crop types (Tab. 3; detailed list in Tab. 1A). The varieties Loras (KWS-Lochow, formerly Lochow-Petkus), a spring type, and Zhongyou 821, a Chinese semiwinter type (Li et al., 1999c), served as susceptible and partially resistant reference line, respectively.

Tab. 3 Crop types of the Brassica napus accessions used for screening resistance against Sclerotinia sclerotiorum.

\begin{tabular}{ll}
\hline Crop type & Number \\
\hline Winter oilseed rape & 127 \\
Spring oilseed rape & 40 \\
Semi-winter oilseed rape & 14 \\
Kale and swede & 16 \\
Brassica leafy vegetable & 1 \\
Synthetic Brassica napus & 1 \\
\hline Total & 199 \\
\hline
\end{tabular}

Seeds were sown in quartz sand and grown in a climate chamber at $16 \mathrm{~h}$ photoperiod and a temperature of $22^{\circ} \mathrm{C} \pm 2{ }^{\circ} \mathrm{C}$. Two ten-day-old seedlings were planted into each pot $(9 \times 9 \times 11 \mathrm{~cm})$ with soil-sand mixture $(1: 3)$ and kept in the same climate chamber for 28 days. Subsequently, winter oilseed rape was kept under short day conditions $(8 \mathrm{~h}$ photoperiod) at $4^{\circ} \mathrm{C}$ in a climate chamber (Mytron WB $750 \mathrm{KFL}$, Mytron Bio- und Solartechnik GmbH, Heilbad Heiligenstadt, Germany) for 8 weeks for vernalisation, while fodder rape, kale and swede were vernalised for 10 weeks. After vernalisation, plants were transferred into bigger pots $(11 \times 11 \times 12 \mathrm{~cm})$ containing one single plant. Spring type oilseed rape plants were directly transplanted into bigger pots after 28 days. The pots were 
placed in a randomised strip block design with 20 plants per line. Plants were kept in a greenhouse until flowering, providing a $14 \mathrm{~h}$ photoperiod at a light intensity of $130 \mu \mathrm{mol}$ $\mathrm{m}^{-2} \mathrm{~s}^{-1}$ at leaf level due to artificial light (Phillips SON-T PIA Plus $400 \mathrm{~W}$, Phillips GmbH Market DACH, Hamburg, Germany). Temperature was maintained at $22^{\circ} \mathrm{C} \pm 2{ }^{\circ} \mathrm{C}$, relative humidity was above $90 \%$. Daily watering was carried out depending on the demand of the plants. During the growth period, plants were fertilised biweekly with $3 \mathrm{~g} \mathrm{Hakaphos}^{\circledR}$ blau (15\% N, 10\% $\mathrm{P}_{2} \mathrm{O}_{5}, 15 \% \mathrm{~K}_{2} \mathrm{O}, 2 \% \mathrm{MgO}$ and micronutrients: $\mathrm{B}, \mathrm{Cu}, \mathrm{Mn}, \mathrm{Mo}, \mathrm{Zn}, \mathrm{Fe}, \mathrm{COMPO}$ Expert $\mathrm{GmbH}$, Münster, Germany) per litre water. These conditions were kept constant until the end of the experiment. Due to limited room in the greenhouse the experiment was split into four runs, with max. 50 accessions each. The reference lines Loras and Zhongyou 821 were included in every run for normalisation of the data from four runs.

An in vitro root inoculation experiment was conducted as a pre-test for tissue specific responses to infection of S. sclerotiorum. The highly susceptible genotype Loras and the partially resistant genotype Zhongyou 821 were used. Seeds were surface-sterilised by washing in $0.1 \%$ silver nitrate for $10 \mathrm{~min}$, then rinsed twice in $\mathrm{dH}_{2} \mathrm{O}$. For germination, seeds were placed on moist filter paper in a Petri dish and kept at $4^{\circ} \mathrm{C}$ over night before temperature was increased to $22^{\circ} \mathrm{C}$ with a $14 \mathrm{~h}$ day period at light intensity of $80 \mu \mathrm{mol} \mathrm{m}$ ${ }^{2} \mathrm{~s}^{-1}$ for 3 days. Seedlings were transferred to a square Petri dish with PNM-medium covered with nylon membrane Sefar Nitex 03-70/33, placing them in one row in the upper third of the Petri dish. From now on, plates stood upright to enable a straight growth of the seedling towards the light (Fig. 5). Twenty plates à six plants were prepared for each genotype.
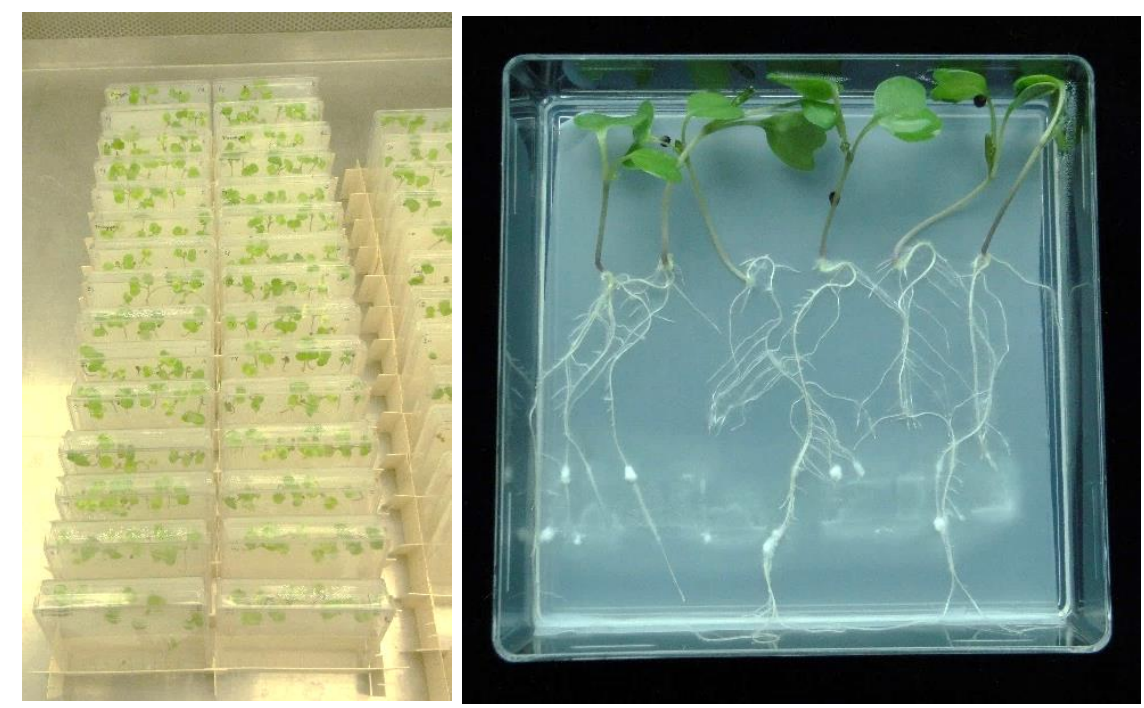

Fig. 5 In vitro test for root inoculation of oilseed rape seedlings in the climate chamber with line-up of plates (left) and arrangement of seedlings (right). Mycelium of S. sclerotiorum is visible in the lower part of the plate. 
For studies on plant tissue specific responses to infection with S. sclerotiorum, an in planta experiment was conducted. Disease development was recorded (see 2.5 Disease assessment in greenhouse experiments), and samples were taken for histological and biochemical studies (see 2.7 Histological studies and 2.8 Biochemical studies).

The following experiments were performed on flowering plants in the greenhouse. Stem, leaves and root were inoculated, as well as the stem base. To avoid being mistaken, in this study the stem base is named 'hypocotyl' which normally refers only to seedlings but is identical to the stem base in plants that already reached vegetative growth.

To assess the response of stem and hypocotyl of oilseed rape to infection of S. sclerotiorum, seeds of Loras and Zhongyou 821 were sown in multi-pot trays filled with standard rooting medium. When first true leaves emerged, 64 plants per genotype, plant tissue and inoculation were transferred into pots $(11 \times 11 \times 9 \mathrm{~cm})$ containing a soil-sand-mixture (3:1).

For assessment of root responses to infection, single seeds were sown directly into pots $(11 \times 11 \times 9 \mathrm{~cm})$ containing a soil-sand-mixture (3:1). In total, 54 plants per genotype, plant tissue and inoculation were cultivated. Plants were grown in the greenhouse under same conditions as described for the disease screening experiment, by providing a $14 \mathrm{~h}$ photoperiod at a temperature of $22^{\circ} \mathrm{C} \pm 2{ }^{\circ} \mathrm{C}$ and a relative humidity above $90 \%$, with regular watering and fertilisation.

Experiments on the leaves were conducted for disease assessment as well as for examinations of tissue specificity. Twenty-two contrasting genotypes from previous resistance screening were selected, including Loras and Zhongyou 821. Twenty plants per genotype were cultivated as above with each two plants in one pot until the second leaf was fully expanded. Same greenhouse conditions as described above were maintained.

In a separate experiment, glucosinolates were analysed in different lines to achieve a broad evaluation of the potential impact of glucosinolates in the different plant parts, stem and root, on the fungal infection. Six lines having shown different responses to stem-inoculation by S. sclerotiorum in the previous screening were selected from the diversity set: SWU Chinese 3, Karat, Furax, Magma, Westar, Duplo. In addition, the reference lines Loras and 
Zhongyou 821, as well as Zhongshuan 9, were included in the test. Twelve plants per genotype and treatment were cultivated in the greenhouse as described above.

\subsection{Cultivation of fungi and inoculation procedures}

Inoculation of the stem, hypocotyl or root took place when $60 \%$ of plants from one line were flowering. An aggressive isolate Ss1.5, collected from infected B. napus plants in Biestow (Mecklenburg-West Pomerania, Germany) was used for inoculation. A sclerotium stored in sterile and dry conditions below $10^{\circ} \mathrm{C}$ was placed on a PDA plate. This mother plate was kept at room temperature to produce fresh mycelium. After 5 to 10 days, agar plugs overgrown with mycelium were cut out with a cork borer $(\varnothing 5 \mathrm{~mm})$ and transferred to new PDA plates to gain pure cultures. After 4 days, agar plugs were punched out from the outer ring of the actively growing mycelium. Using a sterile inoculation needle, the stem or hypocotyl of each plant was injured superficially by puncturing the epidermis. With contact to the mycelium overgrown side, an agar plug was placed on the plant surface at the site of injury. Agar plugs were fixed to the plant by wrapping with Parafilm ${ }^{\circledR}$. To inoculate the tap root, the pot was carefully removed. The clod of earth was gently divided only as far as necessary to reach the main root. The root was cleaned with water, and after a superficial injury produced with an inoculation needle, the agar plug overgrown with mycelium was attached. To hold it in place, the clod of soil and roots was firmly closed around the tap root. The plant was put back into the pot and all plants were generously watered. For control plants, sterile PDA plugs were used.

For in vitro inoculation of roots, PDA strips overgrown by 3 to 5 day old mycelium of S. sclerotiorum were cut and put upside down just below the roots of 10 day old seedlings on PNM-medium (Fig. 6). Agar strips from a clean PDA-plate were used as control. 


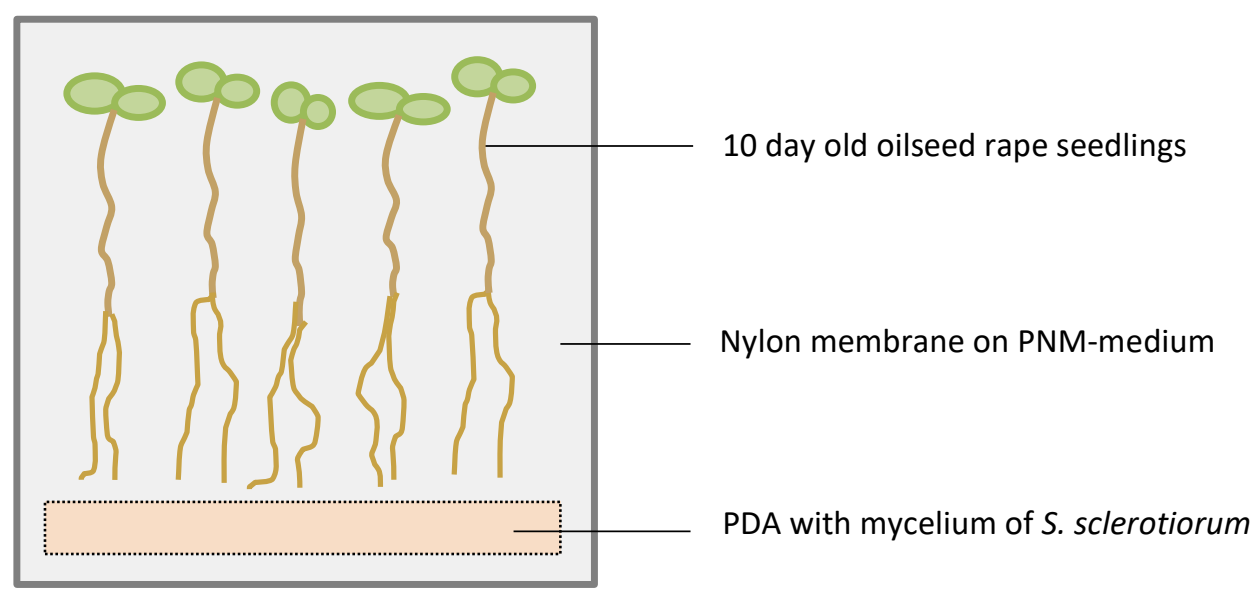

Fig. 6 Design of the in vitro experiment for inoculation with S. sclerotiorum of roots of 10 day old oilseed rape seedlings grown on PNM-medium (Johnson et al., 2011) in a square petri dish.

For tests on the leaf tissue, plants were grown until the second true leaf was fully expanded. Then, the first and second leaf was inoculated by placing an agar plug on one half of the leaf, next to the mid vein (Fig. 7). The plants were then kept under a foil tunnel to maintain humidity and facilitate infection.

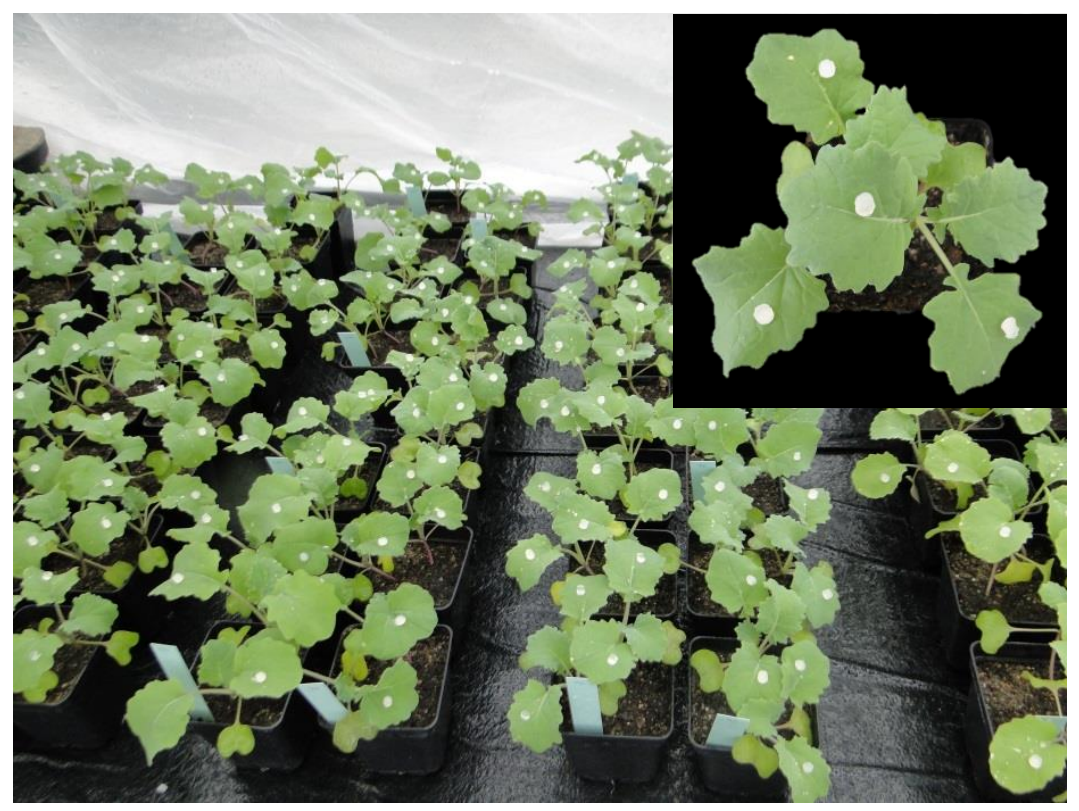

Fig. 7 Different genotypes at the three-leaf stage after agar plug inoculation on the leaves, inoculated in a foil tunnel in the greenhouse. 


\subsection{Disease assessment in greenhouse experiments}

Disease development was examined by measuring the lesion length along the stem with a vernier calliper. For the resistance screening within the diversity set, lesion length was measured at $7 \mathrm{dpi}$. In the experiment of hypocotyl inoculation, lesion measurement was conducted at 1, 3, 5, and $7 \mathrm{dpi}$. In the root inoculation assay, lesion length was measured at 1,4 , and $7 \mathrm{dpi}$. An additional timepoint, $9 \mathrm{dpi}$, was added in the second replication of both root and hypocotyl inoculation experiments. For disease assessment on the leaves, lesion length was measured parallel to the mid vein at 2, 3 and $4 \mathrm{dpi}$. Lesions on steminoculated plants of the GSL-screening experiment were evaluated at $7 \mathrm{dpi}$. Since lesions on stems caused by S. sclerotiorum did not only grow in vertical direction, but also horizontally into the stem, the following assessment key (Tab. 4) was used at $7 \mathrm{dpi}$ to illustrate a full disease development.

Tab. 4 Assessment key for scoring lesions induced by S. sclerotiorum on Brassica species inoculated with the agar plug method.

\begin{tabular}{cl}
\hline Disease score & Lesion development \\
\hline 1 & No expansion beyond inoculation wound visible \\
2 & Lesion dry, shallow and very restricted in expansion \\
3 & Lesion small with wet margin \\
4 & Lesion with a wet margin, elongated and flat, still shallow \\
5 & Lesion as in 4 but growing a little bit deeper in the stem tissue \\
7 & Lesion as in 5 but growing deep into the stem \\
8 & Lesion surrounds 50\% of stem diameter \\
9 & Plant is dying above the lesion \\
\hline
\end{tabular}

\subsection{Quantification of DNA of S. sclerotiorum in plant tissues}

Samples were taken from uninfected plants as well as infected plants, from the area showing a lesion caused by S. sclerotiorum. Three samples of nine plants for each genotype, plant tissue and inoculation were pooled. Samples were lyophilised and milled. Extraction of total DNA from $50 \mathrm{mg}$ of this dry material followed the modified assay of cetyltrimethylammonium bromide (CTAB) method according to Brandfaß \& Karlovsky 
(2008). After homogenisation in $1 \mathrm{ml}$ CTAB with $2 \mu \mathrm{l}$ ß-mercaptoethanol and $1 \mu \mathrm{l} 1 \mathrm{x}$ proteinase $\mathrm{K}$, the mixture was sonicated for $5 \mathrm{sec}$ and incubated at $42^{\circ} \mathrm{C}$ for $10 \mathrm{~min}$, then at $65^{\circ} \mathrm{C}$ for $10 \mathrm{~min}$. To degrade RNA, $1.5 \mu$ RNAse were added and incubated at $37^{\circ} \mathrm{C}$ for $15 \mathrm{~min}$. To isolate DNA, $800 \mu \mathrm{l}$ chloroform:isomyl alcohol (24:1) was added followed by incubation on ice for $10 \mathrm{~min}$. Samples were centrifuged at $12,000 \mathrm{rpm}$ for $10 \mathrm{~min}$, and supernatants mixed in $193.6 \mu \mathrm{l} 30 \%$ PEG and $100 \mu \mathrm{l} 5 \mathrm{M} \mathrm{NaCl}$ for precipitation. Another centrifugation step for 5 min produced a pellet which was washed with $70 \%$ ethanol. The washed pellet was dried in a centrifugal concentrator at $30^{\circ} \mathrm{C}$ for $10 \mathrm{~min}$ and dissolved overnight in $200 \mu \mathrm{l} \mathrm{TE}$ buffer at $4^{\circ} \mathrm{C}$.

The extracted total DNA was stained with ethidium bromide and was qualified with $1 \%$ agarose gel electrophoresis. For the quantitative real-time PCR (qPCR), a highly specific pair of primers being developed for the identification of S. sclerotiorum was used (Tab. 5, Yin et al., 2009). A dilution series from 0.1 to $1000 \mathrm{pg} / \mu \mathrm{l}$ with a dilution factor of 10 was established as standard for qPCR.

Tab. 5 Primer pairs specific for S. sclerotiorum including base sequences and origin used in qPCR.

\begin{tabular}{lllll}
\hline Pathogen & Name & Direction & Base sequence in 5'-3' direction & Reference \\
\hline \multirow{2}{*}{ S. sclerotiorum } & SsF & Forward & AGTCGAGGGACGGGTACTAA & Yin et al., \\
& SsR & Backward & CTTGTCCTCCATTGCCGTTT & 2009 \\
\hline
\end{tabular}

For the PCR, $5 \mu \mathrm{l}$ of amplification mix (qPCRBIO SyGreen Mix Lo-Rox, Nippon Genetics Europe $\mathrm{GmbH}$, Düren, Germany) and $0.4 \mu \mathrm{M}$ of both primers were added to each sample and filled up to a total volume of $10 \mu \mathrm{l}$ with $\mathrm{dd}_{2} \mathrm{O}$. Amplification and melting curve analysis were carried out in a CFX384 real-time PCR detection system (Bio-Rad Laboratories Inc., Hercules, USA) in 384-well plates as described below (Tab. 6). 
Tab. 6 qPCR protocol.

\begin{tabular}{lllll}
\hline Period & Step & Phase & Time & Temperature $\left[{ }^{\circ} \mathrm{C}\right]$ \\
\hline & 1 & Enzyme activation & $2 \mathrm{~min}$ & $95^{\circ} \mathrm{C}$ \\
& 2 & Denaturation & $10 \mathrm{~s}$ & $95^{\circ} \mathrm{C}$ \\
Amplification & 3 & Annealing & $20 \mathrm{~s}$ & $64.5^{\circ} \mathrm{C}$ \\
& 4 & Elongation & $20 \mathrm{~s}$ & $72^{\circ} \mathrm{C}$ \\
& 5 & Steps 2-5 repeated $35 \mathrm{x}$ & & \\
& 6 & Final elongation & $10 \mathrm{~min}$ & $72^{\circ} \mathrm{C}$ \\
Melting curve & 8 & Renaturation & $10 \mathrm{~s}$ & $95^{\circ} \mathrm{C}$ \\
analysis & 7 & Denaturation & $10 \mathrm{~s}$ & $55^{\circ} \mathrm{C}$ \\
& 9 & Melting curve analysis & $\left(0.5^{\circ} \mathrm{C} / 5 \mathrm{~s}\right)$ & $65-95^{\circ} \mathrm{C}$ \\
& 10 & End & $2 \mathrm{~min}$ & $15^{\circ} \mathrm{C}$ \\
\hline
\end{tabular}

For each treatment, PCR was performed with three biological and three technical replicates.

Data were analysed using CFX Manager Software (Bio-Rad laboratories Inc.).

\subsection{Histological studies}

In the histological studies, the colonisation strategy of S. sclerotiorum in different plant tissues of oilseed rape was investigated.

\subsubsection{Sampling and fixation of plant material}

Samples for histological studies were taken from a zone were plant defence mechanisms were expected to occur. Hence, $1 \mathrm{~cm}$ was cut from both the upper and the lower part of the zone including the expanding edge of the lesion, taking $5 \mathrm{~mm}$ of the part being already covered by the lesion and $5 \mathrm{~mm}$ of green and healthy plant material (Fig. 8). Since control plants had no expanding lesions, the inoculation point was used as a benchmark. 


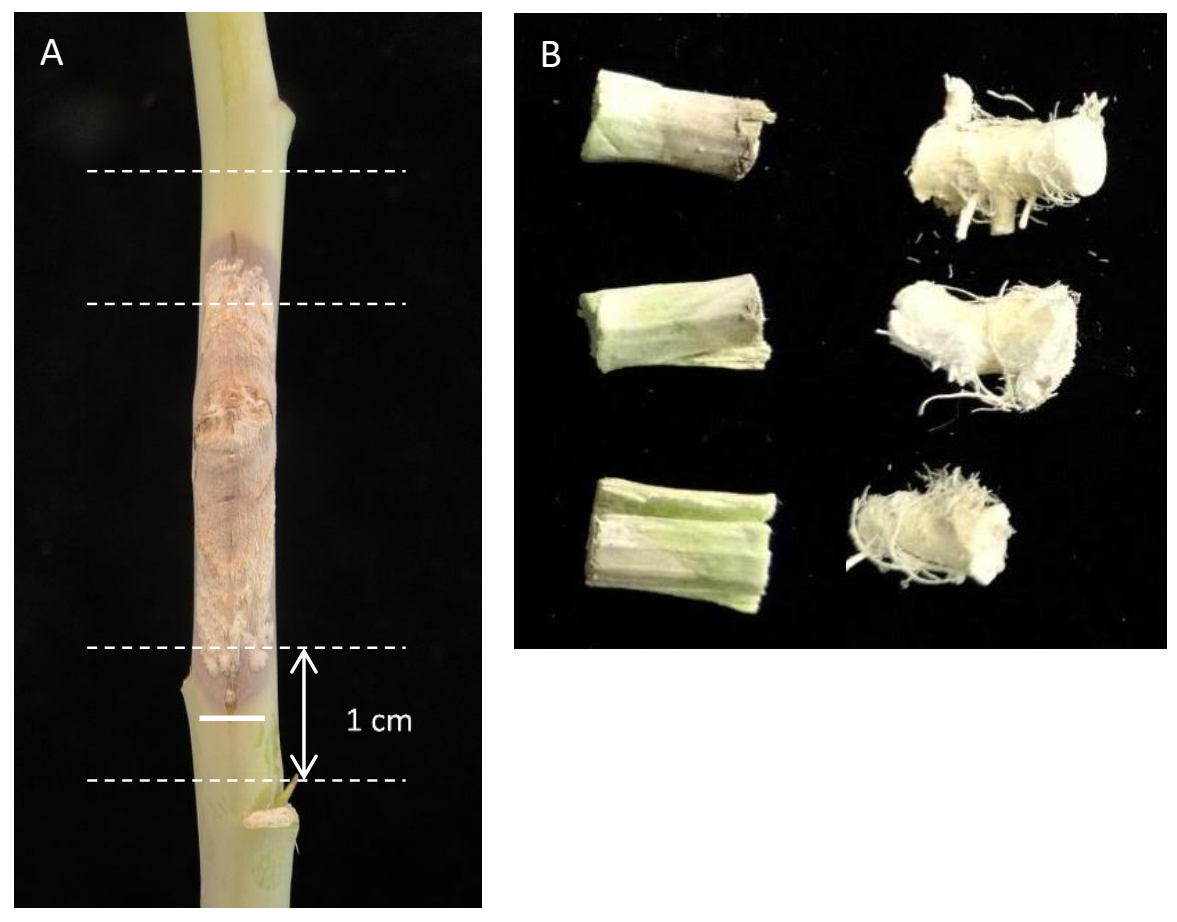

Fig. 8 Sampling scheme for histological and biochemical studies A: Stem of an oilseed rape plant showing typical lesion caused by Sclerotinia sclerotiorum. White marks show where samples were cut at the zone were plant defence mechanisms are expected to occur, including $5 \mathrm{~mm}$ above and below the edge of the lesion B: Freeze-dried samples of infected stems and roots showing lesions.

The sections were immediately immersed in acetic acid-formaldehyde-ethanol solution (AFE), and kept at $4^{\circ} \mathrm{C}$ under constant gentle slewing for $24 \mathrm{~h}$. Then, samples were transferred into $70 \%$ ethanol and kept at $4^{\circ} \mathrm{C}$. Each sampling was accompanied by disease assessment (see 2.5 Disease assessment).

\subsubsection{Paraffin embedding}

After fixation, samples of oilseed rape stem, hypocotyl and root were infiltrated with paraffin to enable thin sectioning for microscopy. Each sample was placed in one embedding cassette (Fig. 9). For dehydration and infiltration, cassettes containing the samples were placed successively in a jar where they were covered the following media (Tab. 7). 
Tab. 7 Procedure for dehydrating and embedding plant material in paraffin.

\begin{tabular}{lll}
\hline Medium & \multicolumn{2}{l}{$\begin{array}{l}\text { Temp- } \\
\text { erature }\end{array}$} \\
\hline PBS & RT & over night \\
$50 \%$ ethanol & RT & $2 \mathrm{~h}$ \\
$70 \%$ ethanol & RT & $2 \mathrm{~h}$ \\
$96 \%$ ethanol & RT & $2 \mathrm{~h}$ \\
Ethanol/Roti ${ }^{\circledR}$-Histol 1:1 & RT & over night \\
Pure Roti ${ }^{\circledR}$-Histol & RT & $2 \times 4 \mathrm{~h}$ \\
Warm saturated Roti ${ }^{\circledR}$-Histol/paraffin & $45^{\circ} \mathrm{C}$ & over night \\
Pure paraffin & $60^{\circ} \mathrm{C}$ & $24 \mathrm{~h}$ \\
Pure paraffin & $60^{\circ} \mathrm{C}$ & $24 \mathrm{~h}$ \\
\hline
\end{tabular}

$\mathrm{RT}$ - room temperature

As long as procedure took place at room temperature, the jars were gently kept in motion to permit homogenous and complete infusion of the plant tissue. As soon as proceeding to higher temperatures, jars were placed in a heating cabinet (Memmert, GmbH \& Co. KG, Schwabach, Germany). The paraffin never solidified until the end of the procedure.

Finally, cassettes were taken consecutively from the molten paraffin, and the samples were immediately placed in fresh molten paraffin in the embedding mould using warm tweezers. The cassette was pressed on the embedding mould (Fig. 9) then being filled up with paraffin and allowed to cool down to solidify.

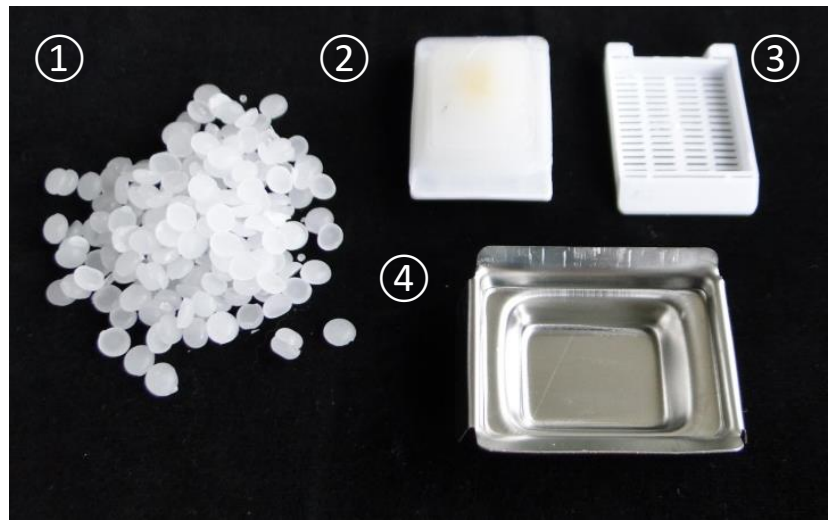

Fig. 9 Material for Paraffin embedding (1) Paraffin pellets (2) cassette with sample embedded in paraffin (3) empty cassette and (4) embedding mould. 
Samples were cut with a rotary microtome (HM 335 E, Microm GmbH, Walldorf, Germany) in cross and longitudinal sections of $14 \mu \mathrm{m}$ (Fig. 10).
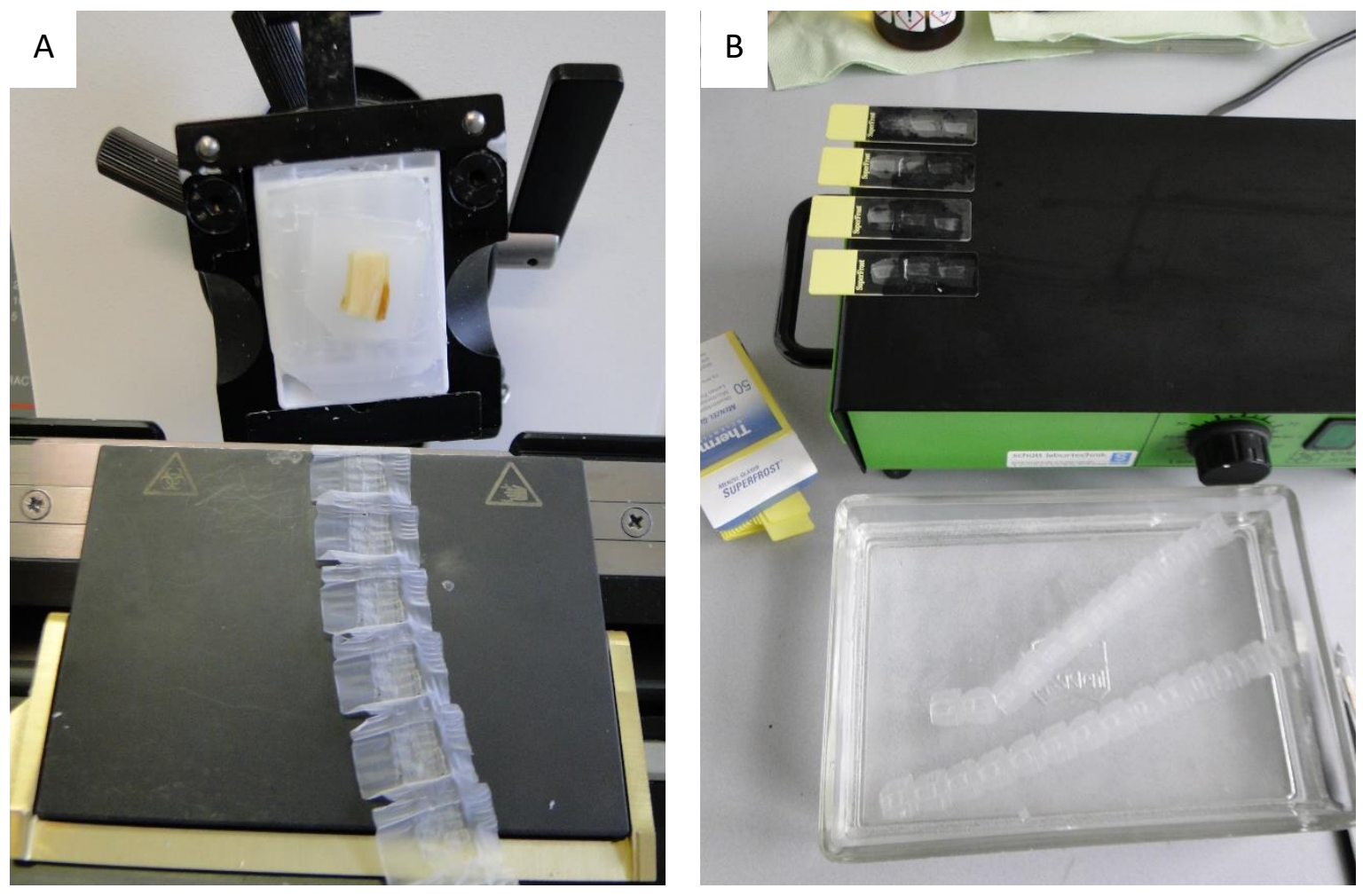

Fig. 10 Microtome cutting of paraffin embedded oilseed rape samples A: Paraffin band of hypocotyl B: Paraffin band floating on water and drying on a warm heating plate.

After transferring the paraffin bands in a cold water bath, they were driven on specimen slides and placed on a moderately warmed heating plate, so that the paraffin bands including plant sections could settle on the glass surface and dry (Fig. $10 \mathrm{~A}$ and B). Sections attached to the specimen slides were submerged into Roti ${ }^{\circledR}$-Histol to be deparaffinised and rehydrated by immersing in decreasing ethanol steps just before staining (Tab. 8). 
Tab. 8 Procedure of deparaffination and rehydration of paraffin embedded sections on specimen slides before staining.

\begin{tabular}{ll}
\hline Solution & Time \\
\hline Roti $^{\circledR}$-Histol & $30 \mathrm{~min}$ \\
$96 \%$ ethanol & $5 \mathrm{~min}$ \\
$90 \%$ ethanol & $5 \mathrm{~min}$ \\
$70 \%$ ethanol & $5 \mathrm{~min}$ \\
$50 \%$ ethanol & $5 \mathrm{~min}$ \\
Bidistilled water & $5 \mathrm{~min}$ \\
\hline
\end{tabular}

\subsubsection{Histochemical staining and microscopy}

\section{Following of fungal growth in plant tissue}

Pianese assay (Gerlach, 1984)

Deparaffinised and rehydrated sections on specimen slides were covered with two to three drops of Pianese staining solution and incubated for $40 \mathrm{~min}$. Then, $92 \%$ ethanol was added, to clear the slides. For differentiation, $92 \%$ ethanol with $0.5 \%$ acetic acid were used. Finally, the slides were carefully rinsed with $\mathrm{dH}_{2} \mathrm{O}$. Specimen were mounted in water and fungal growth in plant tissue observed under the light microscope (Leica DM 500 CS, Leica Microsystems GmbH, Wetzlar, Germany).

Alexa Fluor $488+$ propidium iodide assay

On each of the deparaffinised and rehydrated sections, $30 \mu \mathrm{l}$ of $50 \mu \mathrm{g} / \mathrm{ml}$ Alexa Fluor were added and incubated for $40 \mathrm{~min}$ in a vacuum chamber. The vacuum was interrupted every $10 \mathrm{~min}$ to allow complete infiltration of the tissue. Then, $30 \mu \mathrm{l}$ of $10 \mu \mathrm{g} / \mathrm{ml}$ propidium iodide were added and stained for 15 min under vacuum. Specimen slides were carefully cleaned with $\mathrm{dH}_{2} \mathrm{O}$ and mounted in $50 \%$ glycerol. Confocal laser scanning microscopy (CLSM) was carried out on a Leica TCS SP2 Laser Scanning Microscope 510 (Leica Microsystems CMS $\mathrm{GmbH}$, Mannheim, Germany). For Alexa Fluor the $488 \mathrm{~nm}$ excitation line of the argon laser was used at an intensity of $30 \%$. Signals were collected through a filter block with 500 to $540 \mathrm{~nm}$ emission BP filter. For propidium iodide, the $514 \mathrm{~nm}$ emission line of the argon laser at $30 \%$ intensity was used. Signals were collected through a filter block with 580 to 
$660 \mathrm{~nm}$ BP emission filter. To enhance emission, the 561 emission line of DPSS laser was activated at $30 \%$. Z-series images were collected at $0.63 \mu \mathrm{m}$ intervals through the specimen. Images were processed using the Leica Application Suite Advanced Fluorescence (LAS AF) software.

Chlorazol black E (CBE) assay (after Wilcox \& Marsh, 2009)

To show hyphal growth in fresh leaves, samples were soaked in ethanol overnight. After a washing step with water, leaves were treated with $10 \%$ potassium hydroxide at $90^{\circ} \mathrm{C}$ for 3 to 4 hours, followed by another washing step with water. Then, cleared leaves were incubated in $\mathrm{CBE}$ staining solution at $60^{\circ} \mathrm{C}$ overnight and finally distained in $50 \%$ glycerol. Specimen were observed under the light microscope (Leica DM 500 CS, Leica Microsystems GmbH, Wetzlar, Germany).

\section{Observations of callose deposition}

Trypan-aniline blue double stain assay (Bhadauria et al., 2010)

Deparaffinised and rehydrated sections on specimen slides were treated with $30 \mu \mathrm{l}$ of $0.05 \%$ aqueous trypan blue solution each and incubated overnight at room temperature. Subsequently, 0.05\% aniline blue solution (in $150 \mathrm{mM} \mathrm{KH}_{2} \mathrm{PO}_{4}$ ) was added and incubated for $3 \mathrm{~h}$. Specimen were rinsed with $\mathrm{KH}_{2} \mathrm{PO}_{4}$ and mounted in $50 \%$ glycerol. Observation under a fluorescence microscope (Leica DM 500 CS, Leica Microsystems GmbH, Wetzlar, Germany) mounted with fluorescence filter for aniline blue (excitation at UV range, 340 to $380 \mathrm{~nm}$, suppression LP 425, dichromatic mirror 400) to show callose deposition.

Periodic acids Schiff's assay

Deparaffinised and rehydrated sections on specimen slides were oxidised in oxidation agents for $20 \mathrm{~min}$. The slides were rinsed three times with water. Specimens were covered with Schiff's reagent which was allowed to operate for $10 \mathrm{~min}$. Another three-fold step of rinsing with water was followed by individual addition of $\mathrm{SO}_{2}$-water in case stronger differentiation was needed. Sections were mounted in water and observed under the light microscope (Leica DM 500 CS, Leica Microsystems GmbH, Wetzlar, Germany). 


\section{Observations of tyloses:}

Toluidine blue O stain after Sakai (Gerlach, 1984)

Specimen slides were placed in a staining tray filled with $0.1 \%$ toluidine blue stain and incubated for $20 \mathrm{~min}$. Then, specimen slides were rinsed with $\mathrm{dd}_{2} \mathrm{O}$ and deparaffinised for 30 min in Roti ${ }^{-}-$Histol. Afterwards, specimen slides were rinsed and mounted in $\mathrm{ddH}_{2} \mathrm{O}$ and tyloses were observed under light microscope (Leica DM 500 CS, Leica Microsystems GmbH, Wetzlar, Germany).

\subsection{Biochemical studies}

\subsubsection{Sampling and preparation of plant material}

In each experiment, sampling was done as described before (Fig. 8) and took place after lesion length was recorded. For each biochemical compound, at least four replicates per genotype, plant tissue, dpi and inoculation were analysed, consisting of samples pooled from three plants. Samples were immediately immersed in liquid nitrogen and stored at $-80^{\circ} \mathrm{C}$. Depending on the kind of studies, the material was either freeze-dried and milled (for analyses of glucosinolates and phenolic compounds) or milled with liquid nitrogen when still fresh (for peroxidase activity measurement). Milling was performed with a ball mill (MM 200, Retsch Technology GmbH, Haan, Germany) at a frequency of 30 moves s ${ }^{-1}$ for $1 \mathrm{~min}$ or $30 \mathrm{~s}$, respectively. For each treatment and sampling date, three biological replicates were analysed, where one replicate was composed of three pooled plant samples.

\subsubsection{Quantification of phenolic acids by HPLC}

Phenolic acids synthesised at the beginning of the phenylpropanoid pathway were chosen as representatives commonly considered as key compounds related to lignification or other mechanisms in pathogen defence: $t$-cinnamic acid, $p$-coumaric acid, chlorogenic acid, caffeic acid, ferulic acid, sinapic acid and salicylic acid. The protocol was designed to extract conjugated and free forms of all phenolic acids except salicylic acid being only extracted in the free form. Extraction followed protocols described previously (Mattila \& Kumpulainen, 2002; Ayaz et al., 2008; Eynck et al., 2009). 
For free phenolic compounds, $100 \mathrm{mg}$ of freeze-dried plant powder from both stem and hypocotyl were extracted with $2 \mathrm{ml}$ methanol-water mixture $(80: 20)$ including $0.2 \mathrm{mg} / \mathrm{ml}$ 2,6-di-tert-butyl-4-methylphenol at room temperature for $30 \mathrm{~min}$. After the mixture was centrifuged at 1,000 rpm for $10 \mathrm{~min}$ (Sigma 4-16KS, rotor diameter $18 \mathrm{~cm}$; Sigma GmbH, Osterode am Harz, Germany), the supernatant was transferred into a new tube, resuspended and the extraction repeated. Supernatants were combined and evaporated in RCV 2-25-CD plus speed vacuum evaporator with a CT02-50 SR vacuum pump (Martin Christ Gefriertrocknungsanlagen $\mathrm{GmbH}$, Osterode am Harz, Germany) at $30^{\circ} \mathrm{C}$ for $3 \mathrm{~h}$. The residue was dissolved in $1 \mathrm{ml}$ water. To adjust $\mathrm{pH}$ to 2 to $3,0.5 \mu \mathrm{l} 37 \% \mathrm{HCl}$ was added, followed by another extraction step with $1 \mathrm{ml}$ ethyl acetate. The extract was centrifuged at 4,500 rpm for $30 \mathrm{~s}$; supernatant was transferred into a new tube, and the extraction step repeated. Supernatants were combined (ca. 1,700 $\mu \mathrm{l}$ ) and evaporated until dryness in a speed vacuum at $35^{\circ} \mathrm{C}$ for $90 \mathrm{~min}$. The residue was dissolved in $200 \mu \mathrm{l}$ methanol.

The extraction of conjugated phenolics was performed for the pellet remaining from the aqueous methanol extraction of free phenolics. The pellet was hydrolysed in $1 \mathrm{ml} 2 \mathrm{M}$ $\mathrm{NaOH}$ at $95^{\circ} \mathrm{C}$ for $1 \mathrm{~h}$ and shaken every $10 \mathrm{~min}$. Acidification to $\mathrm{pH} 2$ to 3 was achieved by adding $218 \mu \mathrm{l} 37 \% \mathrm{HCl}$, followed by extraction with $1 \mathrm{ml}$ ethyl acetate and centrifugation at 4,500 rpm for $30 \mathrm{~s}$ to $1 \mathrm{~min}$. Supernatant was transferred into a new tube, and the ethyl acetate extraction step of the pellet repeated. The combined supernatants (ca. 1,700 $\mu$ l) were evaporated until dryness in a speed vacuum at $35^{\circ} \mathrm{C}$ for $90 \mathrm{~min}$. The residue was dissolved in $200 \mu$ methanol.

For a standard mix, 100 ppm of caffeic acid (CA), $p$-coumaric acid (pCA), ferulic acid (FA), sinapic acid (SiA), chlorogenic acid (ChA), trans-cinnamic acid (tCA) and salicylic acid (SA) were dissolved in 80\% HPLC grade methanol. A dilution series with 10 ppm, 7.5 ppm, 5 ppm, 2.5 ppm, 1 ppm, 750 ppb, 500 ppb, 250 ppb, 100 ppb, 75 ppb, 50 ppb were used as internal standards and used for peak assignment for each component by comparing retention times and the UV absorption spectra of analytes to the standards.

For HPLC analysis, all samples or standards were centrifuged at $500 \mathrm{rpm}$ for $5 \mathrm{~min}$ to precipitate undesired particles and prevent injection problems. Then, $80 \mu \mathrm{l}$ of the extraction solution were transferred into HPLC vials. Chromatography was performed on an HPLC-fluorescence/DAD system (Jasco Deutschland GmbH, Pfungstadt, Germany) 
consisting of an AS-2051Plus Intelligent Sampler $\left(4^{\circ} \mathrm{C}\right)$ with a quaternary pump (LG-208004S 04S Quaternary Gradient Unit), a C18 column (EVO-C18 Kinetex, $250 \times 4.6 \mathrm{~mm}, 5 \mu \mathrm{m}$ particle size) protected by a Gemini Nx C18 pre-column $(4 \times 3 \mathrm{~mm}, 5 \mu \mathrm{m})$ in a CO-2060Plus Intelligent Column Thermostat kept at $40^{\circ} \mathrm{C}$, an FP-2020 Plus intelligent fluorescence detector with excitation wavelength at $315 \mathrm{~nm}$ and emission wavelength at $405 \mathrm{~nm}$ and a MD-2015 Plus multi-wavelength detector measuring over the range of 200 to $500 \mathrm{~nm}$. Ten microliters of the solution described above were injected. The analytes were eluted with a mobile phase of different gradients consisting of $(A)$ acetonitrile and (B) water, each containing $0.1 \%$ phosphoric acid, at a flow rate of $1 \mathrm{ml} / \mathrm{min}$ (Tab. 9).

Tab. 9 Protocol of mobile phase gradient chromatography during HPLC analysis.

\begin{tabular}{llll}
\hline Time & Flow rate & Solvent A & Solvent B \\
\hline Pre-run & $1 \mathrm{ml} / \mathrm{min}$ & $87 \%$ & $13 \%$ \\
$5 \mathrm{~min}$ & $1 \mathrm{ml} / \mathrm{min}$ & $87 \%$ & $13 \%$ \\
$29 \mathrm{~min}$ & $1 \mathrm{ml} / \mathrm{min}$ & $64 \%$ & $36 \%$ \\
$29.5 \mathrm{~min}$ & $1 \mathrm{ml} / \mathrm{min}$ & $2 \%$ & $98 \%$ \\
$39.5 \mathrm{~min}$ & $1 \mathrm{ml} / \mathrm{min}$ & $2 \%$ & $98 \%$ \\
$40 \mathrm{~min}$ & $1 \mathrm{ml} / \mathrm{min}$ & $86 \%$ & $14 \%$ \\
$50 \mathrm{~min}$ & $1 \mathrm{ml} / \mathrm{min}$ & $86 \%$ & $14 \%$ \\
\hline
\end{tabular}

Each sample had three technical repeats. Compound identification and quantification was performed by Galaxie chromatography workstation software (Varian Inc., Walnut Creek, California, USA).

\subsubsection{Peroxidase activity assay}

To measure peroxidase activity, $100 \mathrm{mg}$ of freshly ground plant material of each stem, hypocotyl and root, respectively, were used. For enzyme extraction, $2 \mathrm{ml}$ of $50 \mathrm{mM}$ sodium phosphate buffer (SPB) with 1\% polyvinylpyrrolidone were added to the samples being agitated carefully and centrifuged at $15,000 \mathrm{rpm}$ for $30 \mathrm{~min}$ at $4^{\circ} \mathrm{C}$ (Sigma $4-16 \mathrm{KS}$, rotor diameter $18 \mathrm{~cm}$; Sigma GmbH; Osterode am Harz, Germany). Five $\mu$ l of this enzyme extraction solution were transferred into a microtiter plate. Then, $200 \mu$ l of the reaction solution containing $22 \mu \mathrm{l}$ guaiacol, $56.1 \mu \mathrm{l} 35 \% \mathrm{H}_{2} \mathrm{O}_{2}$ and $50 \mathrm{ml} \mathrm{SPB}(25 \mathrm{mM}, \mathrm{pH}$ 7.0) were added and the microtiter plate was kept at room temperature in darkness. After $10 \mathrm{~min}$, 
when the reaction rate was constant, optical density (OD) was measured with a spectrophotometer at $450 \mathrm{~nm}$ (BioTek $\mu$ Quant Monochromatic Microplate Spectrophotometer, BioTek Inc., Winooski, USA). Each sample had three technical replicates.

\subsubsection{Determination of glucosinolates}

Glucosinolate extraction and analysis was done by Dr. Christoph Crocoll, Section of Plant Molecular Biology, Department of Plant and Environmental Sciences, University of Copenhagen. Detailed methodology is described by Crocoll et al., 2016.

Desulfo-glucosinolates were extracted from $10 \mathrm{mg}$ of freeze-dried material and analysed and quantified by an Advance UHPLC system (Bruker, Bremen, Germany) equipped with a C18 reversed phase column (Kinetex $1.7 \mu$ XB-C18 column, $100 \times 2.1 \mathrm{~mm}, 1.7 \mu \mathrm{m}, 100 \AA$, Phenomenex Inc., Torrance, USA). Formic acid in water and acetonitrile $0.05 \%$ were employed as mobile phases $A$ and $B$, respectively. The elution profile was: 0 to $0.5 \mathrm{~min}, 2 \%$ B; 0.5 to $1.2 \mathrm{~min}, 2$ to $30 \% \mathrm{~B} ; 1.2$ to $2.0 \mathrm{~min} 30$ to $100 \% \mathrm{~B}, 2.0$ to $2.5 \mathrm{~min} 100 \% \mathrm{~B}, 2.5$ to 2.6 $\min , 100$ to $2 \%$ B and $2.6-4 \mathrm{~min} 2 \%$ B at a flow rate of $400 \mu \mathrm{l} / \mathrm{min}$. Temperature of column oven was maintained stable between $30^{\circ} \mathrm{C}$ and $40^{\circ} \mathrm{C}$. The liquid chromatography was coupled to an EVOQ Elite TripleQuad mass spectrometer (Bruker, Bremen, Germany) equipped with an electrospray ion source operated in positive ionization mode. The instrument parameters were optimised by infusion experiments with pure standards. The ion spray voltage was maintained at $+3,500 \mathrm{~V}$. Cone temperature was set to $300^{\circ} \mathrm{C}$ and cone gas to 20 psi. Heated probe temperature was set to $400^{\circ} \mathrm{C}$ and probe gas flow to 40 psi. Nebulising gas was set to 60 psi and collision gas to 1.5 mTorr. Nitrogen was used as probe and nebulising gas and argon as collision gas. Active exhaust was constantly on. Multiple reaction monitoring was used to monitor analyte parent ion to product ion transitions. Both Q1 and Q3 quadrupoles were maintained at unit resolution. Bruker MS Workstation software (Version 8.1.2, Bruker, Bremen, Germany) was used for data acquisition and processing. Linearity in ionisation efficiencies were verified by analysing dilution series. Para-hydroxybenzyl glucosinolates was used as internal standard. Each sample had two technical replicates. 


\subsection{Treatment with growth regulators}

This experiment aimed at elucidating the effect of growth regulators on the infection and development of S. sclerotiorum. The experimental setup was similar to greenhouse experiments for disease assessment (see 2.3 Plant materials and cultivation for greenhouse experiments). Four different products were applied. Metconazole (Caramba), paclobutrazol (Toprex) and difenoconazole (Toprex) are fungicides of the triazole group. Mepiquat chloride is a quaternary ammonium compound serving as growth regulator. It is active agent of the product Carax which also contains metconazole. One product only is described as growth regulator: trinexapac-ethyl (Moddus) is a cyclohexanecarboxylate derivative (Tab. 10).

Tab. 10 Active agents, field of application and mode of action of the agrochemical products Caramba, Carax, Moddus and Toprex.

\begin{tabular}{|c|c|c|c|}
\hline Product & Active agent & Field of application & Mode of action \\
\hline Caramba & Metconazole & $\begin{array}{l}\text { Growth regulator } \\
\text { and fungicide }\end{array}$ & $\begin{array}{l}\text { Inhibition of ergosterol synthesis } \\
\text { and gibberellin synthesis }\end{array}$ \\
\hline Carax & $\begin{array}{l}\text { Mepiquat chloride } \\
+ \text { metconazole }\end{array}$ & $\begin{array}{l}\text { Growth regulator } \\
\text { and fungicide }\end{array}$ & $\begin{array}{l}\text { Inhibition of early gibberellin } \\
\text { synthesis }\end{array}$ \\
\hline Moddus & Trinexapac-ethyl & Growth regulator & $\begin{array}{l}\text { Inhibition of late gibberellin } \\
\text { synthesis }\end{array}$ \\
\hline Toprex & $\begin{array}{l}\text { Paclobutrazol + } \\
\text { difenoconazole }\end{array}$ & $\begin{array}{l}\text { Growth regulator } \\
\text { and fungicide }\end{array}$ & $\begin{array}{l}\text { Inhibition of ergosterol synthesis } \\
\text { and gibberellin synthesis }\end{array}$ \\
\hline
\end{tabular}

When plants started stem elongation at a plant age of 4 to 5 weeks (BBCH 32), 30 plants each of Loras and Zhongyou 821 were treated with Caramba, Carax, Moddus, Toprex or water as control, respectively. Application rate adequate to the field amount was applied in a spraying cabinet (Tab. 11). 
Tab. 11 Calculation of growth regulator product volume for spray mixture application in a spray cabinet related to field application.

\begin{tabular}{lcccccc}
\hline Product & $\begin{array}{c}\text { Al } \\
{[\mathrm{g} / \mathrm{l}]}\end{array}$ & $\begin{array}{c}\text { Al at max. field } \\
\text { application rate } \\
{[\mathrm{g} / \mathrm{ha}]}\end{array}$ & $\begin{array}{c}\text { Circular } \\
\text { area } \mathrm{m}^{2}\end{array}$ & $\begin{array}{c}\text { Amount of } \\
\text { application } \\
{[\mu \mathrm{g}]}\end{array}$ & $\begin{array}{c}\text { Amount } \\
\text { for } 33 \mathrm{ml} \\
{[\mu \mathrm{g}]}\end{array}$ & $\begin{array}{c}\text { Volume of } \\
\text { product } \\
{[\mu \mathrm{l}]}\end{array}$ \\
\hline Caramba & 60 & 90.0 & 0.34 & $3,079.07$ & $4,417.80$ & 73.63 \\
Carax & 160 & 224.3 & 0.34 & $7,673.74$ & $11,010.15$ & 68.73 \\
Moddus & 228 & 342.0 & 0.34 & $11,700.48$ & $16,787.65$ & 73.63 \\
Toprex & 125 & 62.5 & 0.34 & $2,138.25$ & $3,067.92$ & 24.54 \\
\hline
\end{tabular}

$\mathrm{Al}$ - active agent

As soon as $60 \%$ of the plants were flowering, $\mathrm{BBCH}$ was determined and the plant height and stem diameter at the inoculation point were measured. Plants were inoculated, and measurement of lesion length took place at 3, 5 and $7 \mathrm{dpi}$, as described above.

\subsection{Statistical analyses}

Data were statistically analysed using the software Statistica (Version 13, StatSoft, TulsaOklahoma, USA). Multifactorial ANOVA followed by Fisher test was applied on data showing normal distribution and homogeneity of variance. Data sets not normally distributed and lacking homogenous variance were tested using a non-parametric KruskalWallis ANOVA and considered significant at $P<0.05$. Analyses of correlation followed the Spearman correlation coefficient. 


\section{Results}

\subsection{Screening Brassica germplasm for genotypic resistance by stem inoculation}

Disease assessment of S. sclerotiorum on different B. napus crop types was run on 199 accessions in total. The chosen method required an elongated stem and reaching the flowering stage for inoculation and disease assessment. Not all genotypes developed the corresponding features and instead showed an atypical phenotype (Fig. 11, Tab. 1A). Due to the abnormally slower development of stem and flower of some genotypes, the internodes of these plants became too tough for inoculation. While normally plants were flowering 10 to 14 weeks after sowing (Fig. $11 \mathrm{~A}$ ), several genotypes remained in the vegetative state (e.g. Lesira and Parapluie, Fig. 11 B and C). Those took a longer time to produce a stem and they meanwhile lost leaves due to senescence, without inflorescence having emerged. When the stem was long enough to technically allow inoculation, it turned out to be very tough. Such genotypes did not belong to a certain growth type. The majority of these plants showed a reduced lesion length $(<15 \mathrm{~mm})$ and was attributed to a lower disease score $(<4)$. Several accessions belonging to the swedes did not produce an elongated stem but generated a turnip like root instead (Jaune à collet vert, Fig. 11 D). There were also accessions showing very weak growth types with only single leaves occurring (Bienvenue DH4, Fig. $11 \mathrm{E}$ ) or a reduced plant height, thin stems and premature flowering (e.g. wild accession, swedes like Sensation NZ, Tina and VIGE DH1). These were highly susceptible to $S$. sclerotiorum. 

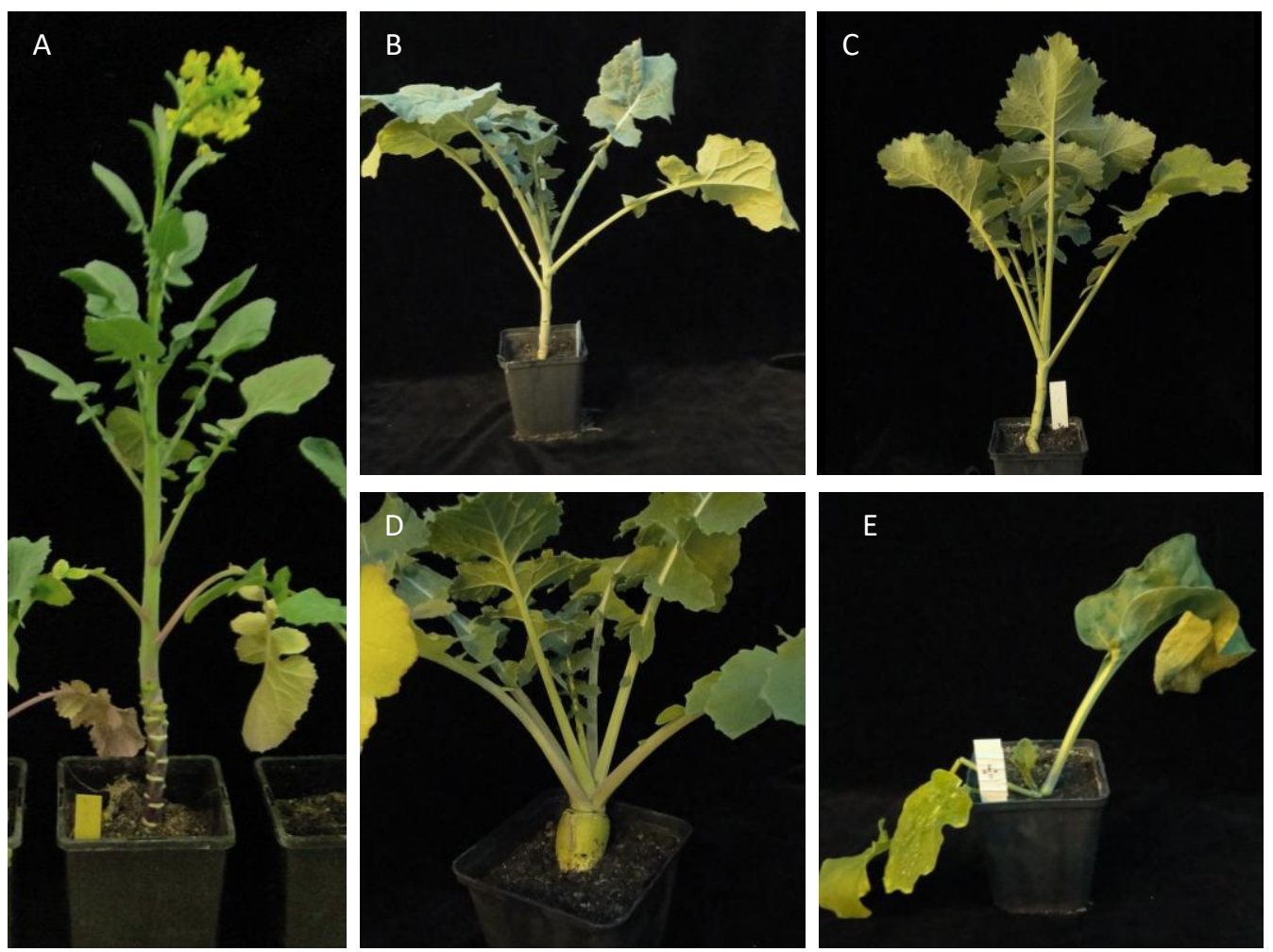

Fig. 11 Examples for atypical phenotypes of different oilseed rape (OSR) genotypes not appropriate for inoculation $\geq 20$ weeks after sowing A: Zhongyou 821 (semi-winter OSR) B: Lesira (spring OSR) C: Parapluie (winter fodder) D: Jaune à collet vert (swede) and E: Bienvenue DH4 (winter OSR) with A: Normal development until flowering stage with stem elongation B,C: Genotypes remain in vegetative stage D: No stem elongation E: Weakly growing genotype lacking typical phenotype of OSR.

Therefore, only 183 genotypes could be included in the phenotyping for resistance against S. sclerotiorum. Among the successfully screened 183 genotypes no potential source of resistance was found (Fig. 12). 


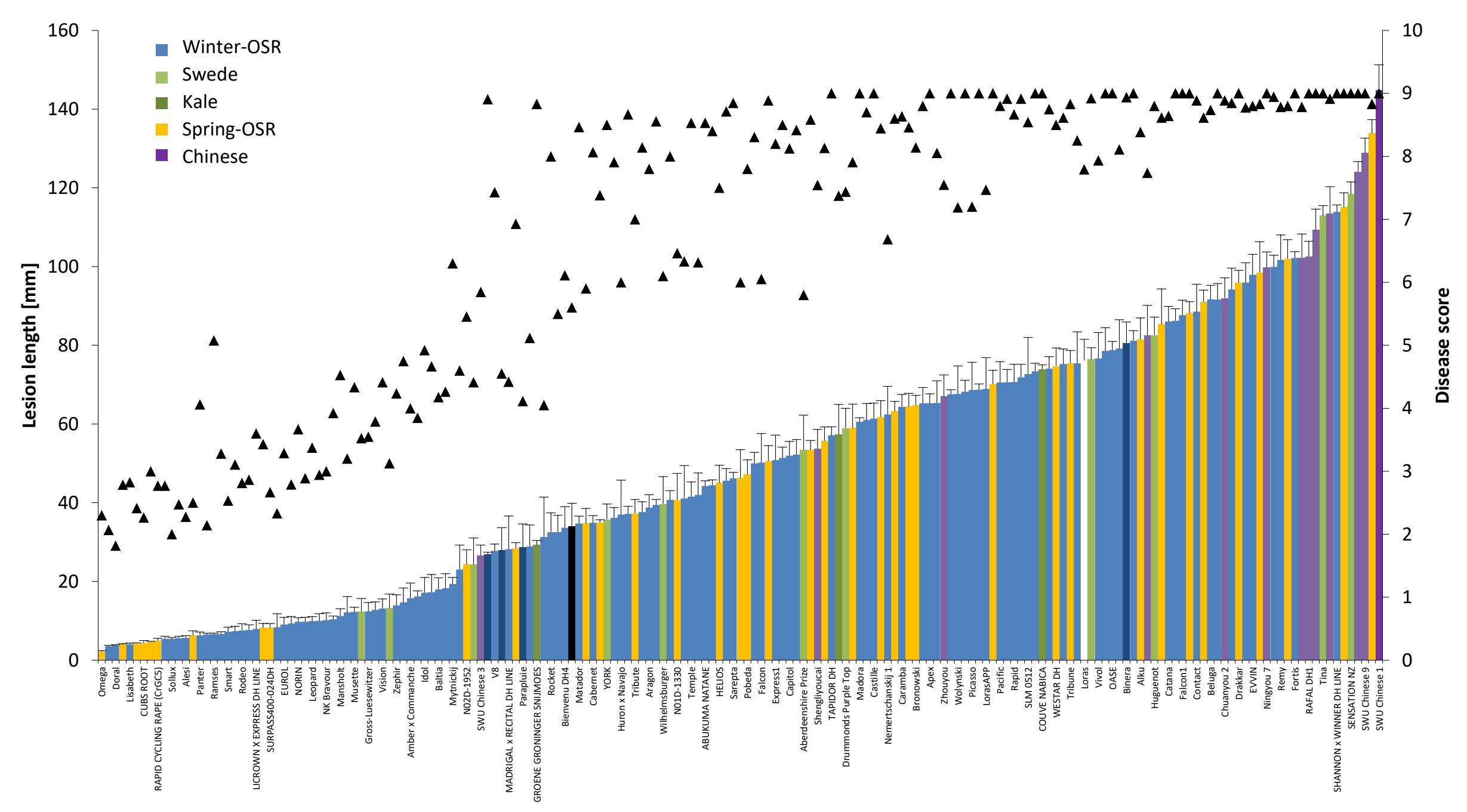

Fig. 12 Lesion length and disease score $(\mathbf{\Lambda})$ at 7 days post inoculation with S. sclerotiorum on stems of 183 growth types of $B$. napus. Reference genotypes Loras (susceptible) and Zhongyou 821 (partially resistant) are indicated as white and black bar, respectively. Mean values and standard error are shown ( $\mathrm{n}=20$ ). 
All tested accessions showed disease symptoms after the inoculation of S. sclerotiorum. No accession performed symptoms rated with a score lower than 2 for a dry, i.e. restricted lesion growth attributed to resistance. A low score of 2 only occurred in 10 genotypes. Among those only one line, Ceska Krajova, performed the normal genotype. Most of the other accessions showing low values for both lesion length and disease score did not flower and had either a very tough or no stem at all, as described above.

Disease scores of the genotypes developing an elongated stem and reaching flowering stage mainly were assigned to intermediate to high scores. The following table (Tab. 12) shows that only minor numbers of accessions had low (1-4) or intermediate scores (5-7), and most were assessed susceptible $(>7)$, thus showing that the stem was largely covered by the lesion.

Tab. 12 Number and portion of accessions classified as less susceptible, intermediate or susceptible to $S$. sclerotiorum according to disease score.

\begin{tabular}{llcc}
\hline Disease score & Classification & Number of accessions & Portion [\%] \\
\hline $1-2$ & resistant & 0 & 0 \\
$3-4$ & less susceptible & 39 & 21 \\
$5-7$ & intermediate & 43 & 23 \\
$8-9$ & susceptible & 101 & 55 \\
\hline Total: & & $\mathbf{1 8 3}$ & $\mathbf{1 0 0}$ \\
\hline
\end{tabular}

All in all, 83\% of the screened accession showed lesion scores higher than 3.5 indicating higher susceptibility. Lesion length ranged from 2.2 to $144.9 \mathrm{~mm}$, where $77.9 \%$ of the genotypes showed a lesion longer than $9.5 \mathrm{~mm}$, reaching binary values and showing advanced progress of disease.

The results for lesion length and disease score of the different growth types were equally distributed. Lesion length and score were highly correlated with $R_{S}=0.87(P<0.01)$. The reference genotypes Loras and Zhongyou 821 were considered as highly susceptible and partially resistant but were not significantly different from one another. Comparing the disease assessment data from all accessions to the reference genotypes, Zhongyou 821 was significantly different to accessions with a lesion length higher than $90 \mathrm{~mm}$ and a score of 9, but not to accessions with lower values for both parameters compared to itself (Tab. 2A). 
Loras differed significantly from accessions with lesion length smaller than $30 \mathrm{~mm}$ and a disease score of 2.5 but was not different from accessions with longer lesions than Loras itself. The two reference genotypes Loras and Zhongyou 821, considered as susceptible and partially resistant, did not show strictly consistent response to the inoculation in all four runs (Fig. 13). The first and the fourth run delivered the expected results. Genotypic differences between Loras and Zhongyou 821 were significantly distinct from each other, with the susceptible Loras suffering from clearly longer and deeper lesions than Zhongyou 821, which is partially resistant. However, in run two and three the results were contrary but less or not significant.

In all experiments, control plants did not develop any lesions.

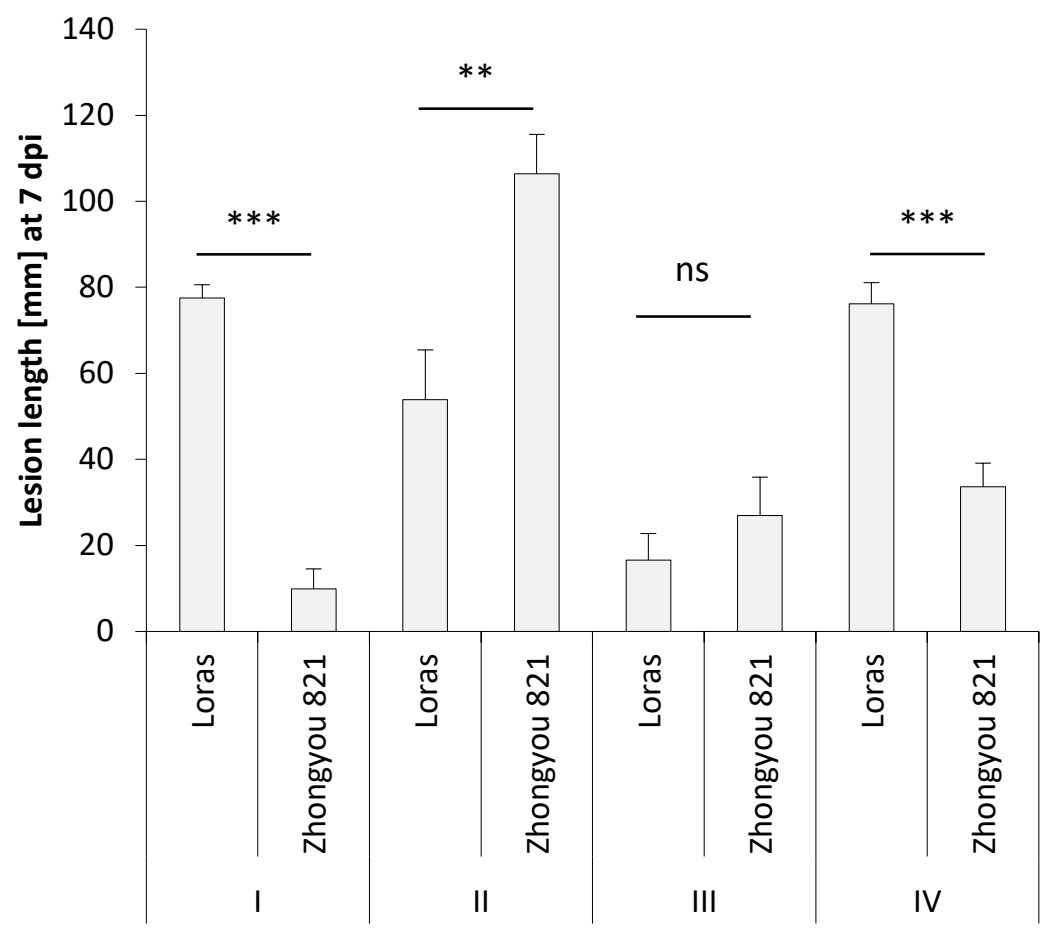

Fig. 13 Lesion length in OSR genotypes Loras (susceptible) and Zhongyou 821 (partially resistant) 7 days post inoculation with S. sclerotiorum in four different runs (I-IV). Mean values and standard errors are shown. Asterisks mark significant differences (Kruskal-Wallis ANOVA: $\mathrm{n}=20 ;{ }^{*} P<0.05,{ }^{* *}$ $P<0.01$ and $* * * P<0.001)$. 


\subsection{Disease assessment on leaves}

To shorten the screening procedure, a leaf screening was tested as an alternative to disease assessment on stem-inoculated plants. Eighteen accessions varying in lesion length and disease score in the previous stem screening were selected (Tab. 13) and the reference genotypes Loras (susceptible) and Zhongyou 821 (partially resistant) were included in the test, as well as the genotypes Falcon and Express. For the statistics see annex (Tab. 2A).

Tab. 13 Oilseed rape genotypes selected for leaf assessment test with contrasting responses 7 days after stem inoculation and 4 days after leaf inoculation with S. sclerotiorum. Grey lines correspond to less susceptible genotypes after stem inoculation. Mean values are shown.

\begin{tabular}{llcccc}
\hline \multirow{2}{*}{ Number } & Name & \multicolumn{2}{c}{ Lesion length [mm] } & \multicolumn{2}{c}{ Disease score } \\
\cline { 2 - 6 } & & Stem & Leaf & Stem & Leaf \\
\hline BnASSYST-128 & Leopard & 12.5 & 5.0 & 3.9 & 1.0 \\
& Express & 16.7 & 21.7 & 5.3 & 3.4 \\
BnASSYST-029 & Idol & 17.2 & 5.9 & 4.9 & 1.1 \\
BnASSYST-107 & Janetzkis Schlesischer & 17.3 & 12.6 & 4.7 & 1.6 \\
BnASSYST-528 & Vision & 18.4 & 4.8 & 5.1 & 0.8 \\
BnASSYST-096 & Hansen $\times$ Gaspard & 23.1 & 2.7 & 4.6 & 0.5 \\
BnASSYST-2311 & SWU Chinese 3 & 26.6 & 2.1 & 5.9 & 0.4 \\
& Zhongyou 821 & 34.1 & 17.3 & 5.6 & 3.0 \\
BnASSYST-185 & Canard & 26.7 & 0.3 & 8.7 & 0.2 \\
BnASSYST-180 & V8 & 27.1 & 0.4 & 6.7 & 0.1 \\
BnASSYST-2188 & Groene Groninger Snijmoes & 27.8 & 0.3 & 7.8 & 0.1 \\
BnASSYST-5277 & Temple & 35.7 & 0.4 & 7.5 & 0.2 \\
& Falcon & 43.4 & 15.9 & 8.6 & 2.5 \\
BnASSYST-264 & Helios & 45.0 & 0.0 & 7.5 & 0.0 \\
BnASSYST-521 & Palmedor & 73.9 & 0.1 & 8.5 & 0.1 \\
BnASSYST-240 & Westar (DH) & 74.7 & 0.2 & 8.5 & 0. \\
- & Loras & 76.2 & 23.3 & 7.8 & 3.7 \\
BnASSYST-5122 & Catana & 77.9 & 0.0 & 8.1 & 0.0 \\
BnASSYST-098 & Rafal (DH1) & 102.5 & 0.7 & 9.0 & 0.2 \\
BnASSYST-1066 & Shannon $\times$ Winner & 113.9 & 0.0 & 9.0 & 0 \\
BnASSYST-270 & SWU Chinese 9 & 128.9 & 0.6 & 9.0 & 0.2 \\
\hline & Weihenstephaner & 133.9 & 10.0 & 8.8 & 1.5 \\
\hline
\end{tabular}


For leaf symptoms, an assessment key was developed according to symptom expansion on infected leaves. Symptom development on first and second fully expanded leaves was recorded as follows (Fig. 14).

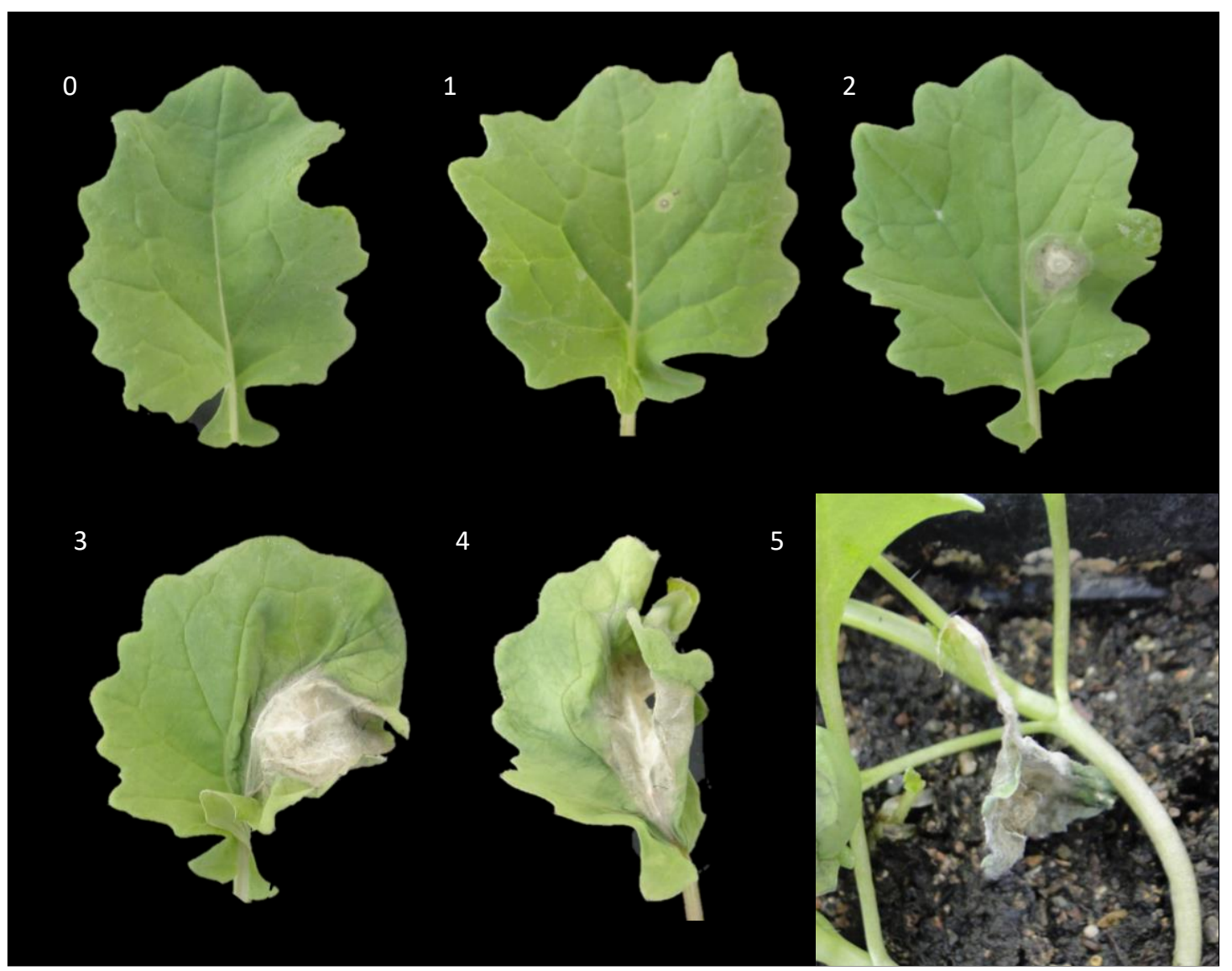

Fig. 14 Symptoms on oilseed rape leaves at the $4^{\text {th }}$ day post inoculation with S. sclerotiorum 0: No symptoms 1: Small and dry lesion 2: Small, water-soaked lesion with round shape 3: Lesion extends to leaf edge or petiole; leaf starts wilting 4: Symptoms spread to the non-inoculated half of the leaf 5: Leaf is entirely destroyed by the lesion.

Leaf inoculation resulted in a broad range of lesion development being assessed the $4^{\text {th }}$ day post inoculation (Fig. 15). Disease development on the first and second leaf of three weeks old plants in two leaf stage was not correlated to that on the stem of flowering plants. On the one hand, genotypes with minor susceptibility to $S$. sclerotiorum tested by stem inoculation did not have the same response on the leaf. On the other hand, genotypes showing high susceptibility in stem screening displayed only small lesions with a low disease score on the leaf. The reference genotype Loras was highly susceptible in the leaf 
as well as in the stem screening. Reference genotype Zhongyou 821 being partially resistant in the stem screening showed high lesion length and score in the leaf screening.

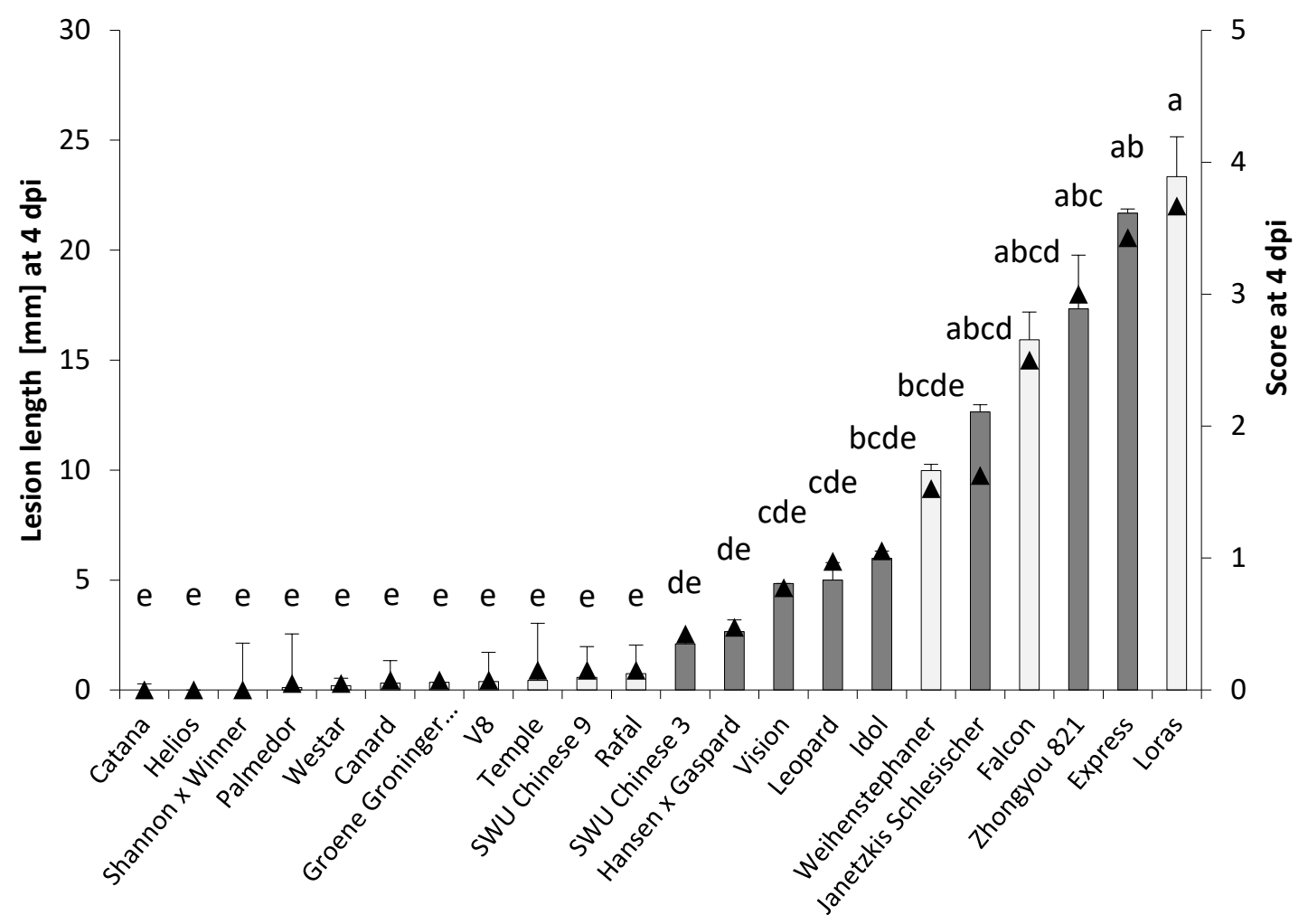

Fig. 15 Lesion length and disease score ( $\mathbf{\Lambda}$ ) 4 days post inoculation with S. sclerotiorum on the first and second leaf of different oilseed rape genotypes. Dark grey bars correspond to less susceptible genotypes based on previously tested stem inoculation. Mean values and standard errors are shown. Letters indicate significant differences in lesion length (Kruskal-Wallis ANOVA: $n=20$, $P<0.05)$.

\subsection{Disease assessment and fungal development on different plant tissues}

Disease assessment was done on two reference oilseed rape genotypes and on three different plant tissues. Measurement of lesion development as well as pathogen quantity took place.

\subsubsection{Symptom development}

Lesion development after inoculation with S. sclerotiorum was studied in the stem, hypocotyl and root in the two contrasting oilseed rape genotypes Loras (susceptible) and Zhongyou 821 (partially resistant). 


\section{Disease phenotyping on stem and hypocotyl}

Lesion length was growing in the course of time, and differences in the susceptibility of the genotypes were visible with Loras exhibiting larger lesions than Zhongyou 821 (Fig. 16).

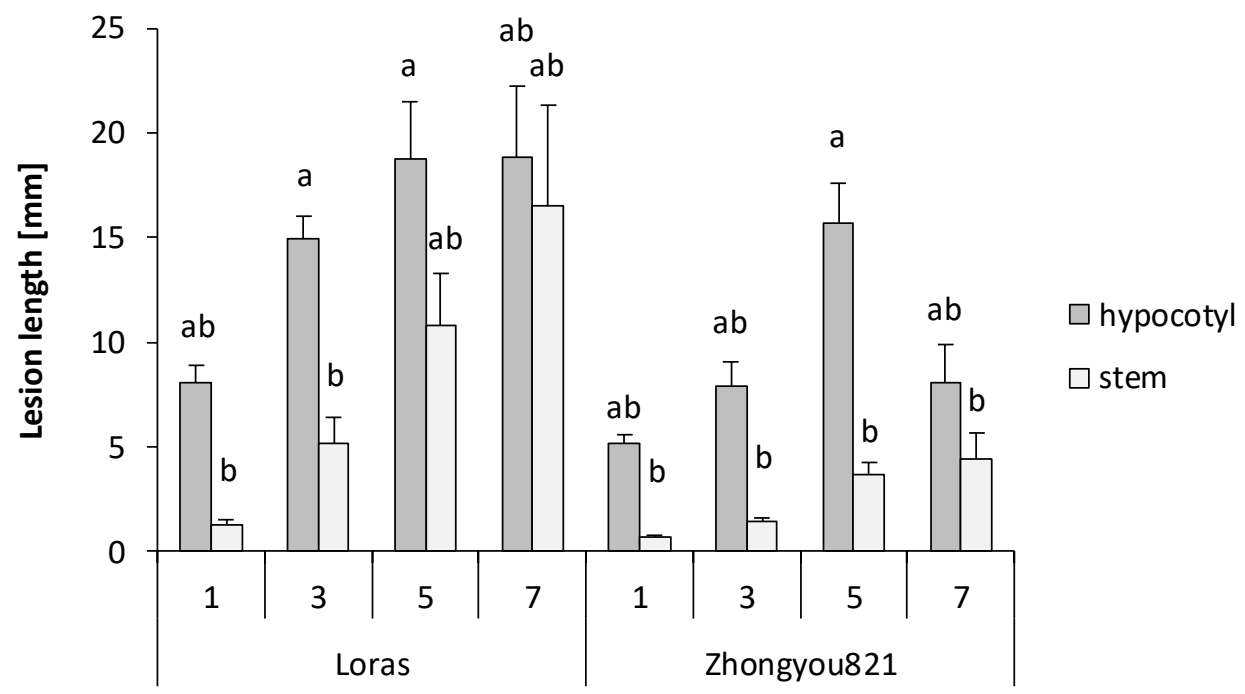

Fig. 16 Lesion length at 1, 3, 5 and 7 days post inoculation with S. sclerotiorum of stem and hypocotyl of the OSR genotypes Loras (susceptible) and Zhongyou 821 (partially resistant). Mean values and standard errors are shown. Letters show significant differences (Kruskal-Wallis ANOVA: $\mathrm{n}=16, P<0.05)$.

In both genotypes, the lesions on the stem were smaller than on the hypocotyl. The differences between genotypes and plant tissues were not significant in most cases being confirmed by main effect test (Tab. 14). Except by fungal infection, no effect on the lesion length was found. 
Tab. 14 F-values of main effects of genotype, inoculation, plant tissue and the interactions on the lesion length including the degrees of freedom (df). Asterisks represent differences according to significance level (Wald test: $n=16 ;{ }^{* *} p<0.001,{ }^{* *} p<0.01,{ }^{*} p<0.05$ and $n s$ not significant).

\begin{tabular}{lll}
\hline Effect & df & Lesion length [mm] \\
\hline Genotype & 1 & $0.35^{\mathrm{ns}}$ \\
Inoculation & 1 & $10.77^{* * *}$ \\
Plant tissue & 1 & $0.68^{\mathrm{ns}}$ \\
Genotype $\times$ Inoculation & 1 & $0.12^{\mathrm{ns}}$ \\
Inoculation $\times$ Plant tissue & 1 & $5.11^{*}$ \\
Genotype $\times$ Plant tissue & 1 & $0.04^{\mathrm{ns}}$ \\
Genotype $\times$ Inoculation $\times$ Plant tissue & 1 & $0.01^{\mathrm{ns}}$ \\
\hline
\end{tabular}

Lesion length was positively correlated with the treatment $\left(R_{s}=0.64\right)$ and with the day post inoculation $\left(R_{s}=0.38\right)$. Furthermore, there was a weak negative correlation of lesions depending on the genotype $\left(R_{s}=-0.12\right)$. At $7 \mathrm{dpi}$, Loras showed mean disease scores of 3.94 and 4.69, respectively. Score of the stem and hypocotyl lesions of Zhongyou 821 averaged at 2.38 and 3.08, respectively (see annex Tab. $3 \mathrm{~A}$ ). No correlation existed between disease score and plant tissue.

The experiment was repeated with mycelium overgrown agar plugs placed on injured and non-injured plants with disease assessment at only $7 \mathrm{dpi}$. There were no consistent and significant differences in the lesion development showing only a tendency of smaller lesions after inoculation without injury. In the injured plants, the lesion expansion was higher in the stem than in the hypocotyl. Disease development and growth of lesion was identical in both genotypes. Lesions were prominently larger than in the first experiment, reaching a maximum length in the stem of $51.6 \mathrm{~mm}$ and $63.2 \mathrm{~mm}$ in Loras and Zhongyou 821, respectively. Disease scores reached 7.19 and 7.14 in the stem and hypocotyl of Loras, and 8.19 and 4.25 in the stem and hypocotyl of Zhongyou 821 (see annex Tab. 4A). No correlation was found between disease score and plant tissue. 


\section{Disease phenotyping on stem and root}

In the course of time, lesion elongation was equally taking place in both tissues, stem and root becoming significantly different between 1 and $7 \mathrm{dpi}$ in the stem of both genotypes (Fig. 17).

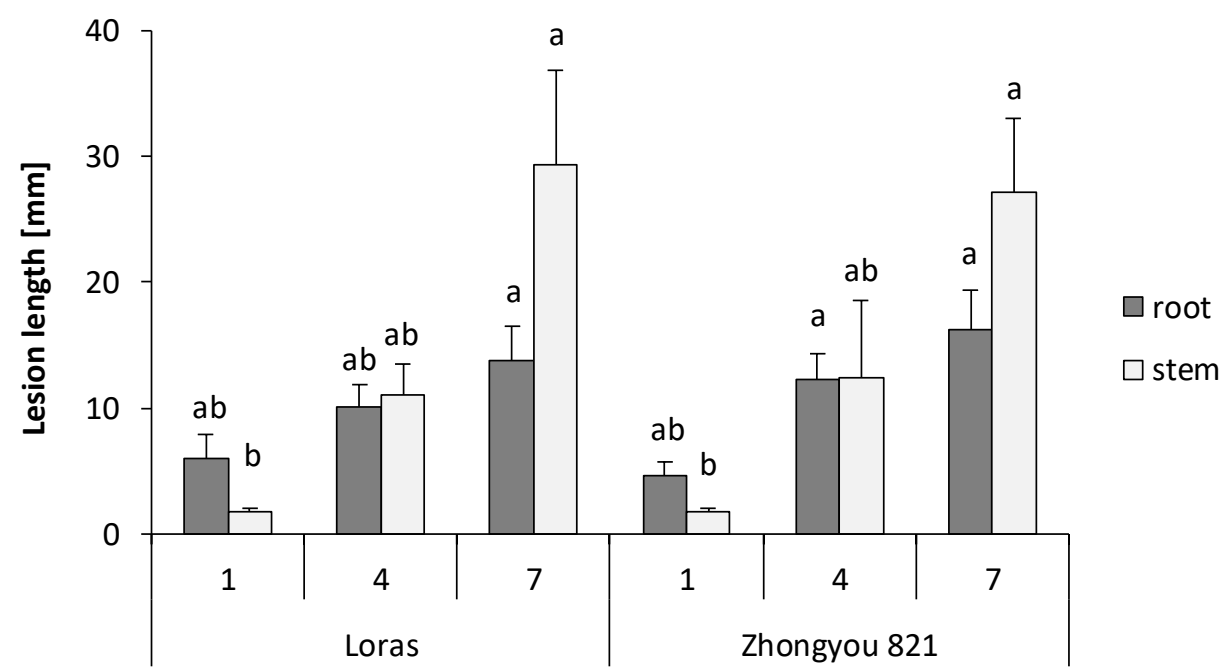

Fig. 17 Lesion length after 1, 4 and 7 days post inoculation with S. sclerotiorum of stem and root of the oilseed rape genotypes Loras and Zhongyou 821. Mean values and standard errors are shown. Letters show significant differences (Kruskal-Wallis ANOVA: $\mathrm{n}=9, P<0.05$ ).

The lesion length and day post inoculation were positively correlated $\left(R_{S}=0.38\right)$. Correlation between lesions and the treatment ranked even higher $\left(R_{S}=0.72\right)$. The effect of inoculation was significant (Tab. 15). In the first days post inoculation, lesion development was not distinguishable between the tissues. Then, while in the stem lesion length was strongly increasing up to $29.3 \mathrm{~mm}$ (Loras) and $27.2 \mathrm{~mm}$ (Zhongyou 821), in the root the lesions grew more slowly, finally reaching approximately half the size of the stem lesion at $7 \mathrm{dpi}$ with a maximum mean of $13.8 \mathrm{~mm}$ and $16.2 \mathrm{~mm}$ in Loras and Zhongyou 821, respectively (see annex Tab. 5A). However, neither genotype nor plant tissue had significant effect on lesion development (Tab. 15). 
Tab. 15 F-values of main effects of genotype, inoculation, plant tissue and the interactions on the lesion length including the degrees of freedom (df). Asterisks represent differences according to significance level (Wald test: $n=9 ;{ }^{* * *} p<0.001,{ }^{* *} p<0.01,{ }^{*} p<0.05$ and ns not significant).

\begin{tabular}{lll}
\hline Effect & df & Lesion length [mm] \\
\hline Genotype & 1 & $0.23^{\text {ns }}$ \\
Inoculation & 1 & $5.02^{*}$ \\
Plant tissue & 1 & $0.47^{\text {ns }}$ \\
Genotype $\times$ Inoculation & 1 & $0.18^{\text {ns }}$ \\
Inoculation $\times$ Plant tissue & 1 & $0.63^{\text {ns }}$ \\
Genotype $\times$ Plant tissue & 1 & $0.22^{\text {ns }}$ \\
Genotype $\times$ Inoculation $\times$ Plant tissue & 1 & $0.24^{\text {ns }}$ \\
\hline
\end{tabular}

Disease scores reached 5.56 and 6.11 in the stem and root of Loras, and 5.33 and 5.89 in stem and root of Zhongyou 821 (see annex Tab. 5A).

When the experiment was repeated, equal results were obtained (see annex Tab. 6A). Like in the first experiment, lesion elongation was quite constant in both, stem and root. In the susceptible genotype Loras, the stem lesion kept developing till later assessment date of $10 \mathrm{dpi}$, being significantly different to $1 \mathrm{dpi}$. Again, differences in the plant tissues were not significant but showed a tendency of longer lesions in the stem than in the root. No differences were observed between genotypes. In both experiments, plant tissue and disease scores were not correlated.

\subsubsection{Quantification of DNA of S. sclerotiorum}

DNA of S. sclerotiorum was quantified in infected stem and root of the OSR genotypes Loras and Zhongyou 821 at $7 \mathrm{dpi}$. In the control, no fungal DNA has been found (Fig. 18). 


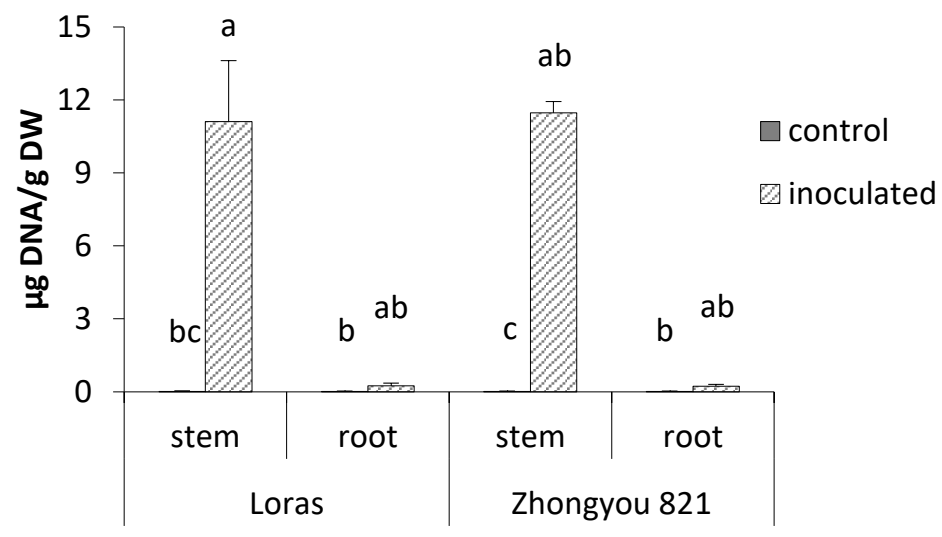

Fig. 18 Amount of fungal DNA in the stem and root of the oilseed rape genotypes Loras and Zhongyou 821 at 7 days post inoculation with S. sclerotiorum. Mean values and standard errors are shown. Letters indicate significant differences (Kruskal-Wallis ANOVA: $n=9, P<0.05$ ).

In the stem of both genotypes, the quantity of DNA was significantly higher than in the control. Seven days after inoculation, only $0.24 \mu \mathrm{g} / \mathrm{g}$ DW of fungal DNA was found in the root. Compared to the root, significantly higher amount of fungal DNA accumulated in the stem. The lesion length caused by S. sclerotiorum and the amount of fungal DNA were positively correlated $\left(R_{s}=0.72\right)$ which was highly significant $(P<0.01)$.

\subsection{Histological studies on different plant tissues}

Different stains were applied to $14 \mu \mathrm{m}$ thin sections of stem, hypocotyl and root as well as entire leaves of Loras (susceptible) and Zhongyou 821 (partially resistant) to allow the examination of hyphal growth of S. sclerotiorum in the different plant tissues of both genotypes. The images of this chapter illustrate that the colonisation patterns clearly distinct between the different plant tissues.

\subsubsection{Histology of the root (in vitro)}

Oilseed rape seedlings were grown and inoculated in vitro. For microscope observation, roots were stained with Alexa Fluor and propidium iodide and examined with the confocal laser scanning microscope (CLSM). Both, roots of the seedlings and mycelium of S. sclerotiorum emitted wavelengths attributed to green and red (Fig. $19 \mathrm{~A}$ and B). 

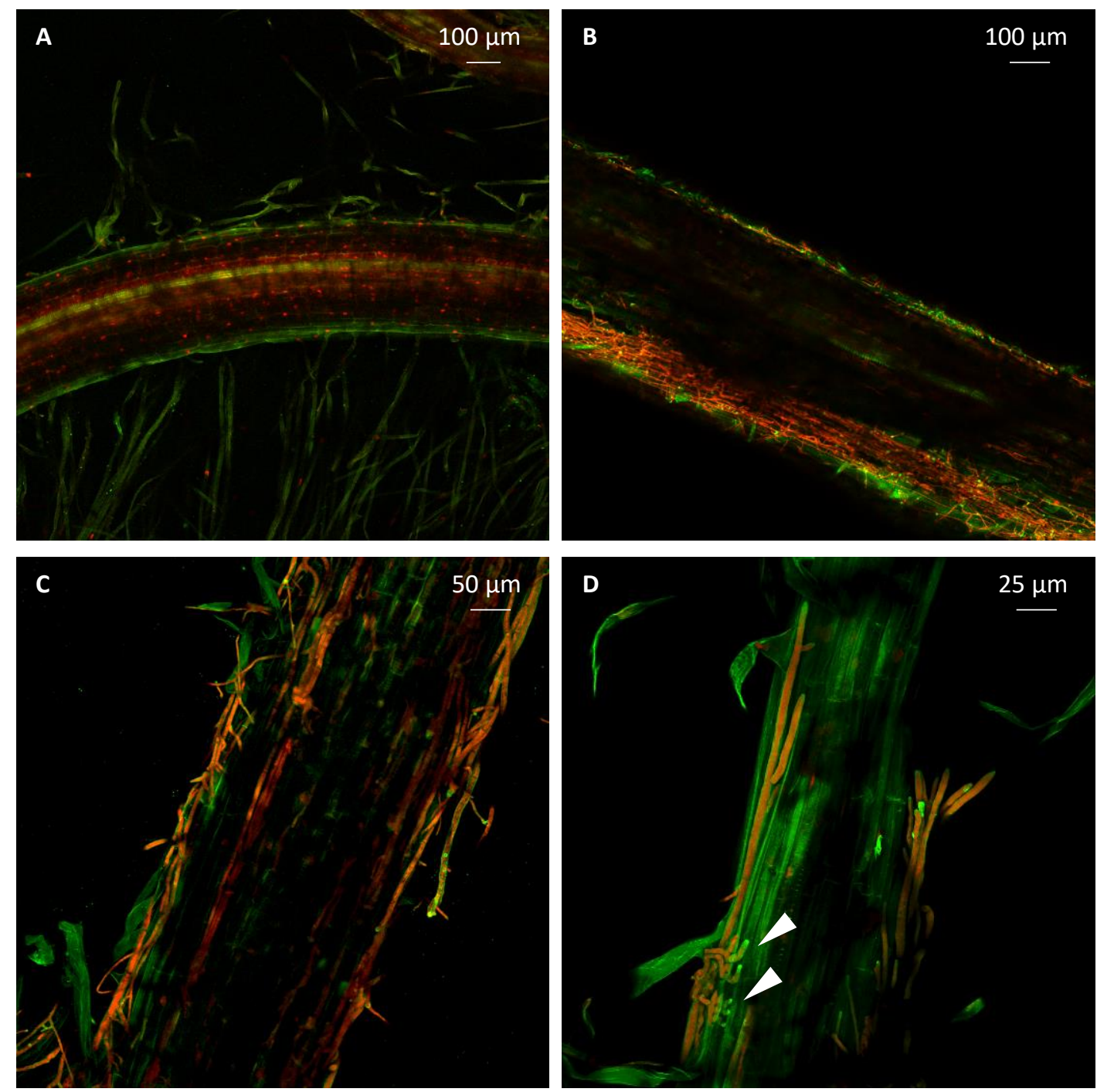

Fig. 19 CLSM images after staining with Alexa Fluor and propidium iodide of 10 days old roots of oilseed rape grown in vitro and infected with S. sclerotiorum A: Non-inoculated root; Loras B: Mycelium growing densely on the root surface; Loras, $24 \mathrm{hpi} \mathrm{C:} \mathrm{Mycelium} \mathrm{growing} \mathrm{densely} \mathrm{on}$ the surface; Zhongyou 821, 24 hpi D: Intensely branching hyphae with infection structures on the root surface (white arrow heads); Zhongyou 821,12 hpi.

Already at 12 hours post inoculation (hpi), the mycelium covered the root surface in a thick and dense layer, and nearly no emission was recorded from the plant cells. At $24 \mathrm{hpi}$, the roots were completely overgrown by the fungus (Fig. $19 \mathrm{~B}$ and C), but special infections structures like infection cushions were not detected. Only seldomly, especially at the outer edge of colonisation, branching hyphae occurred (Fig. 19 D). Those hyphae grouped to form more compact infection structures, seemingly growing into the plant tissue. Apart from this, no hyphae were found within the young roots. 


\subsubsection{Histology of the stem}

The Pianese method was tested on deparaffinised sections (Fig. 20). Pianese stained the plant tissue blueish and fungal cells in purple colour. Hyphae could be located in the plant tissue and the direction of hyphal growth in longitudinal direction along the stem could be shown. Observing the centre of the lesion where plant material was colonised earlier, hyphae were detected in the cortex and the phloem (Fig. 20 A). At the expanding edge of a lesion, hyphae were discovered in the cortex only (Fig. 20 B).
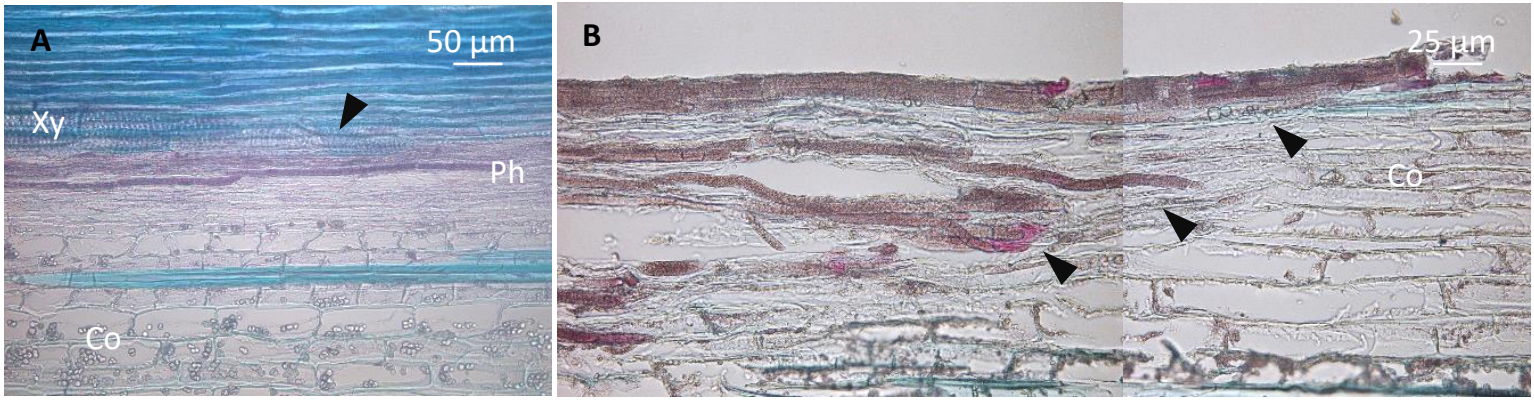

Fig. 20 Light microscopy images of oilseed rape stem sections after infection with Sclerotinia sclerotiorum, stained with Pianese showing purple stained hyphae within blueish plant tissue A: Hyphae advancing within the phloem B: Hyphae advancing sub epidermal and within the cortex. Hyphae are indicated with black arrow heads. Co - cortex, $\mathrm{Ph}-$ phloem, $\mathrm{Xy}-\mathrm{xylem}$.

A double stain of Alexa Fluor 488 and propidium iodide was applied to examine specimen with the confocal laser scanning microscope (CLSM). Images of plant tissue observed with the CLSM mainly appeared in red emission light, but the vascular bundles and sclerenchyma cells could also show green emission light (Fig. 21 A). The fungus reflected wave length to appear red, coated with a clear green layer (Fig. 21 B). 

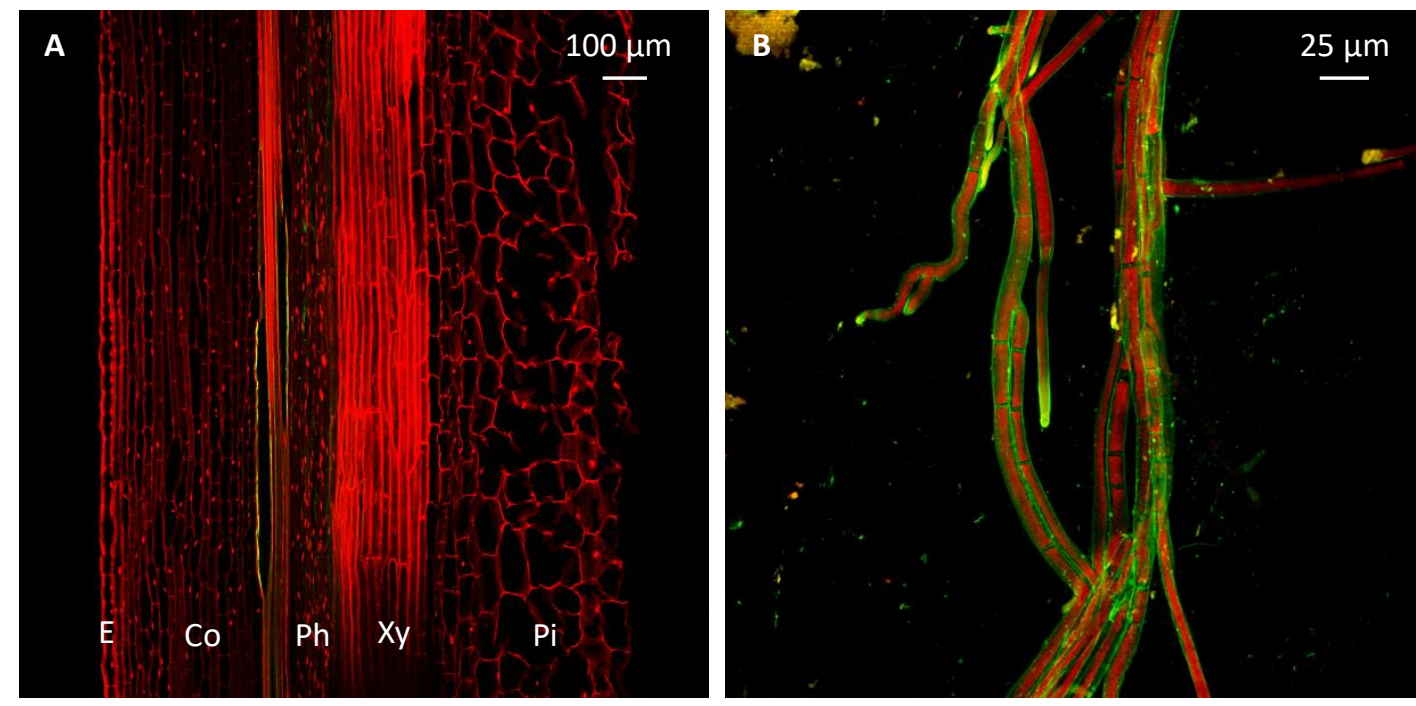

Fig. 21 CLSM images of specimen stained with Alexa Fluor and propidium iodide A: Stem section of a non-inoculated oilseed rape plant; Zhongyou 821 B: Hyphae of S. sclerotiorum. E - epidermis, $\mathrm{Co}$ - cortex, $\mathrm{Ph}$ - phloem, $\mathrm{Xy}$ - xylem, $\mathrm{Pi}$ - pith.

There were no differences in the cell morphology between the two genotypes Loras and Zhongyou 821 (Fig. 22). Observation of numerous sections of different samples revealed no differences between the number or organisation of cell layers and strength of the cell wall size.
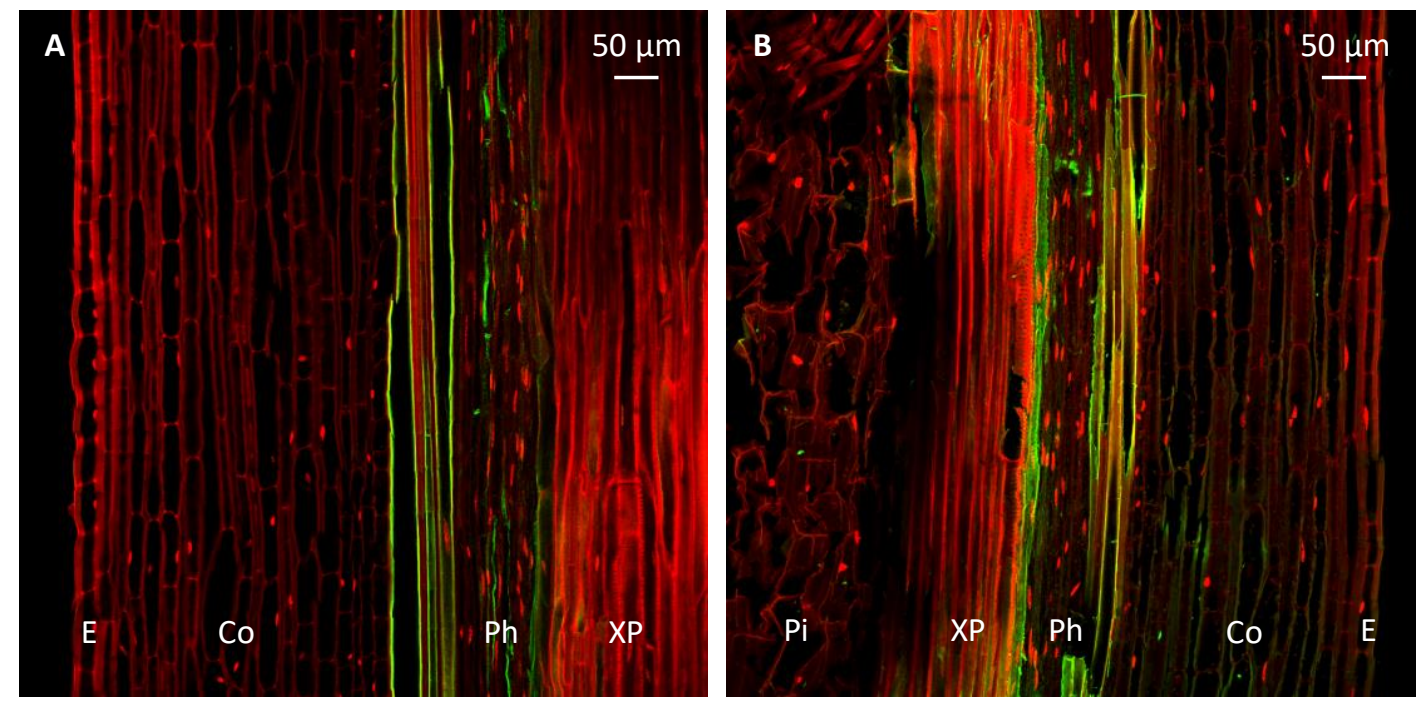

Fig. 22 CLSM images of non-inoculated oilseed rape stem sections of the genotypes A: Zhongyou 821 and B: Loras stained with Alexa Fluor and propidium iodide. E - epidermis, Co - cortex, $\mathrm{Ph}$ - phloem, XP-xylem parenchyma, $\mathrm{Pi}$ - pith.

In general, no differences were observed between the genotypes Loras and Zhongyou 821 neither concerning the cell structure of the plant parts, nor regarding the colonisation pattern of the fungus in the two different genotypes. At the first day post inoculation, 
slightly less hyphae were found but at later infection stages, the colonisation pattern stayed identical over time.

As to be seen in Fig. 23 A and B, some sections of infected plants included a thick layer of mycelium located outside of the plant tissue, accompanied by sub-epidermal growing hyphae inside the plant tissue. Outside of the plant, hyphae were thinner than inside.
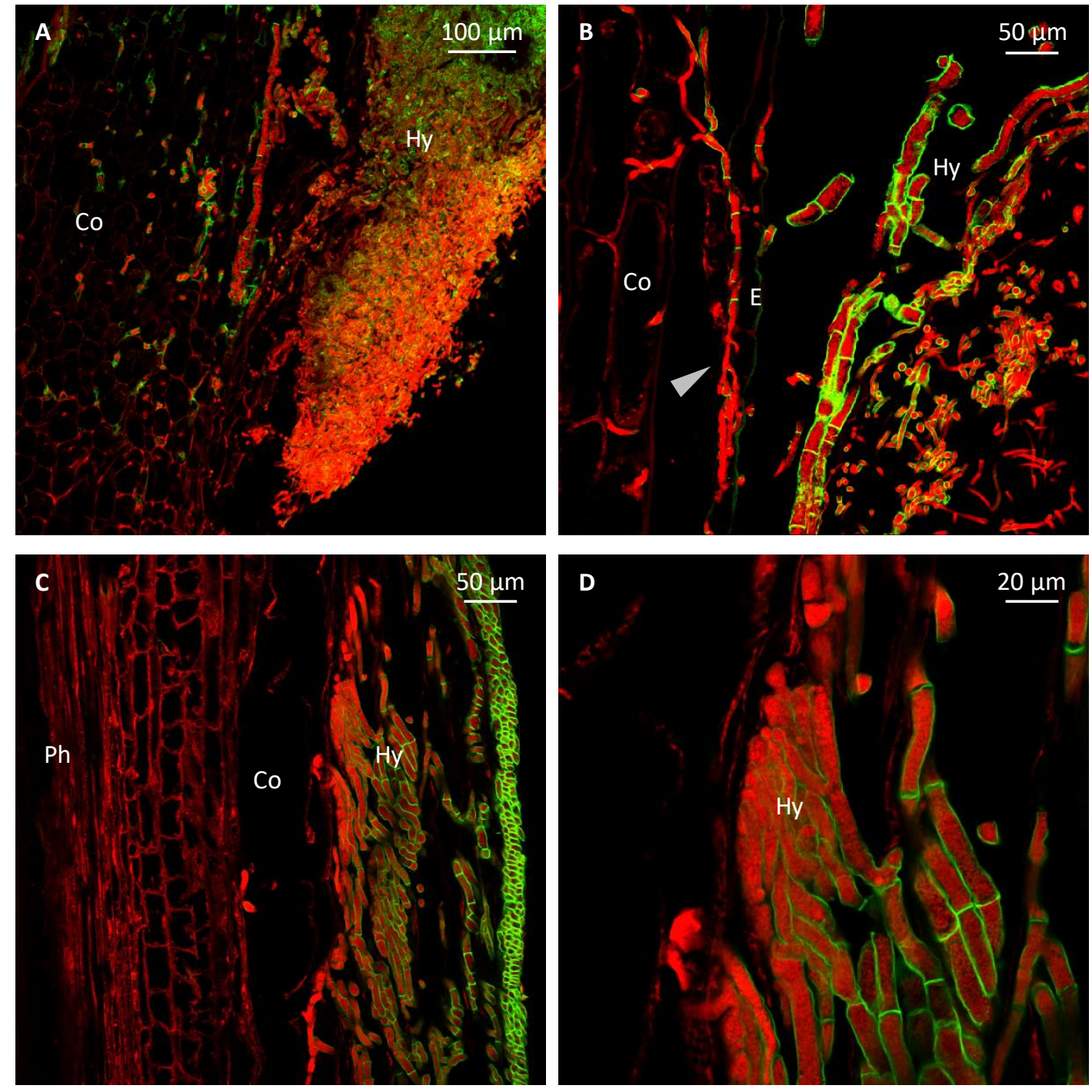

Fig. 23 CLSM imgages showing growth of S. sclerotiorum in stem of oilseed rape (Loras), stained with Alexa Fluor and propidium iodide A: Mycelium mat outside plant tissue; $7 \mathrm{dpi}$ B: Mycelium mat outside plant tissue and sub epidermal hyphae (grey arrow head); $7 \mathrm{dpi} \mathrm{C:} \mathrm{Hyphal} \mathrm{front} \mathrm{in} \mathrm{the} \mathrm{cortex;}$ $3 \mathrm{dpi}$ D: Hyphae front is stopping at plant cell as a barrier; $3 \mathrm{dpi}$. E - epidermis, Co - cortex, $\mathrm{Hy}-$ hyphae, $\mathrm{Ph}$ - phloem.

The hyphal growth within the stem tissue generally was in an aligned way in longitudinal direction along the cells. Here, hyphae formed typical hyphae fronts with the hyphal tips advancing in a row and simultaneously overcoming barriers such as plant cell walls (Fig. 
23 D). Dense accumulation of hyphae was mainly found in the cortex (Fig. 23 C), where as a result the plants cells were barely visible.

Fig. 24 shows the general proceeding of hyphae in the stem. Hyphae especially were found in the cortex, where they advanced in parallel to each other, following longitudinal sense of the cells (Fig. $24 \mathrm{~A}$ and B).
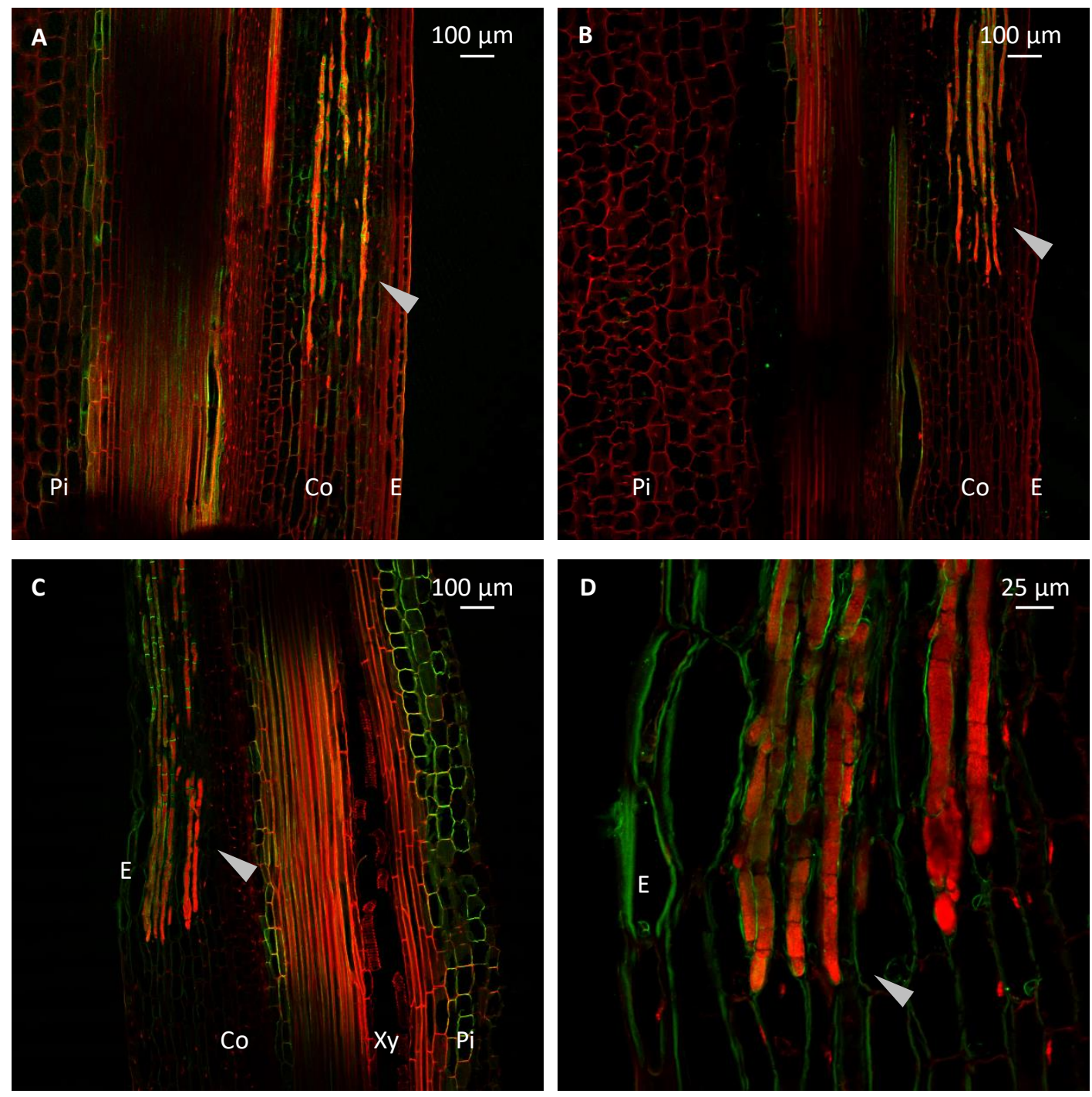

Fig. 24 CLSM imgages showing growth of S. sclerotiorum in the stem of oilseed rape, stained with Alexa Fluor and propidium iodide $\mathbf{A}$ and B: Hyphae (grey arrow head) are assembled in the cortex of Loras (A) and Zhongyou 821 (B), showing parallel, longitudinal growth; $1 \mathrm{dpi} \mathrm{C:} \mathrm{Older} \mathrm{hyphae}$ (grey arrow head) within macerated plant tissue; Zhongyou 821, 3 dpi D: Densely growing hyphae in the cortex and phloem with the xylem staying clear and an intact epidermal cell layer; Zhongyou 821, 3 dpi. E - epidermis, Co - cortex, Ph - phloem, Xy - xylem, Pi - pith.

There were no differences between Loras and Zhongyou 821 in the way hyphae were colonising the tissue. Colonisation of the pith never occurred in the observed samples and infection stages. At infection stages later than $1 \mathrm{dpi}$, cortex tissue got noticeably macerated 
shown by a lesser emission of light due to demoralised cell walls (Fig. 24 C). Focussing on the tips of hyphae it became visible that they advanced intercellularly (Fig. $24 \mathrm{D}$ ). The epidermis stayed intact.

Sections of later infection stages ( $>1 \mathrm{dpi}$ ) show that towards the centre of the lesion, where fungal structures colonised the tissue earlier, the plant tissue was disorganised and macerated (Fig. $25 \mathrm{~A}$ and B). Such regions of tissue exhibited hyphae proceeding into the phloem, but generally not into the xylem vessels (Fig. 25 B). Moreover, hyphae remained mainly in the cortex.
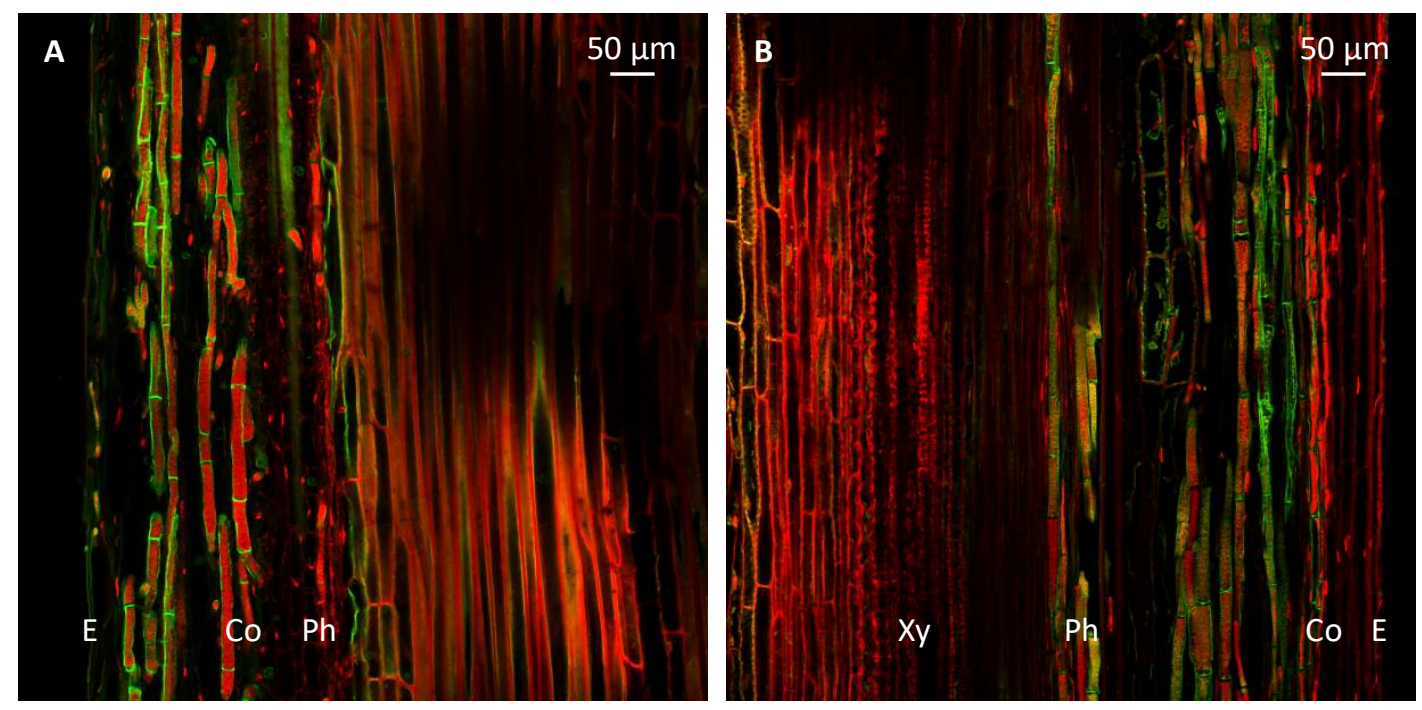

Fig. 25 CLSM imgages showing growth of S. sclerotiorum in stem oilseed rape, stained with Alexa Fluor and propidium iodide A: Hyphae in the cortex and phloem of Zhongyou 821; 3 dpi B: Hyphae in the cortex and phloem of Loras; 9 dpi. E-epidermis, Co - cortex, $\mathrm{Ph}$ - phloem, $\mathrm{Xy}-\mathrm{xylem}$.

\subsubsection{Histology of the hypocotyl}

In the hypocotyl, hyphae showed a different colonisation pattern compared to the stem (Fig. 26). In the hypocotyl, advancing hyphae grew less organised and traversed the tissue in a seemingly undirected way (Fig. $26 \mathrm{~A}$ and B). As in the stem, mycelium within hypocotyl tissue formed hyphae fronts (Fig. 26 C), and also advanced intercellularly (Fig. 26 D). Both pictures show highly vacuolated hyphae. Hyphae also were capable to penetrate the cell wall by forming cell bundles (Fig. $26 \mathrm{E}$ and F). Afterwards, hyphae gain original size. 

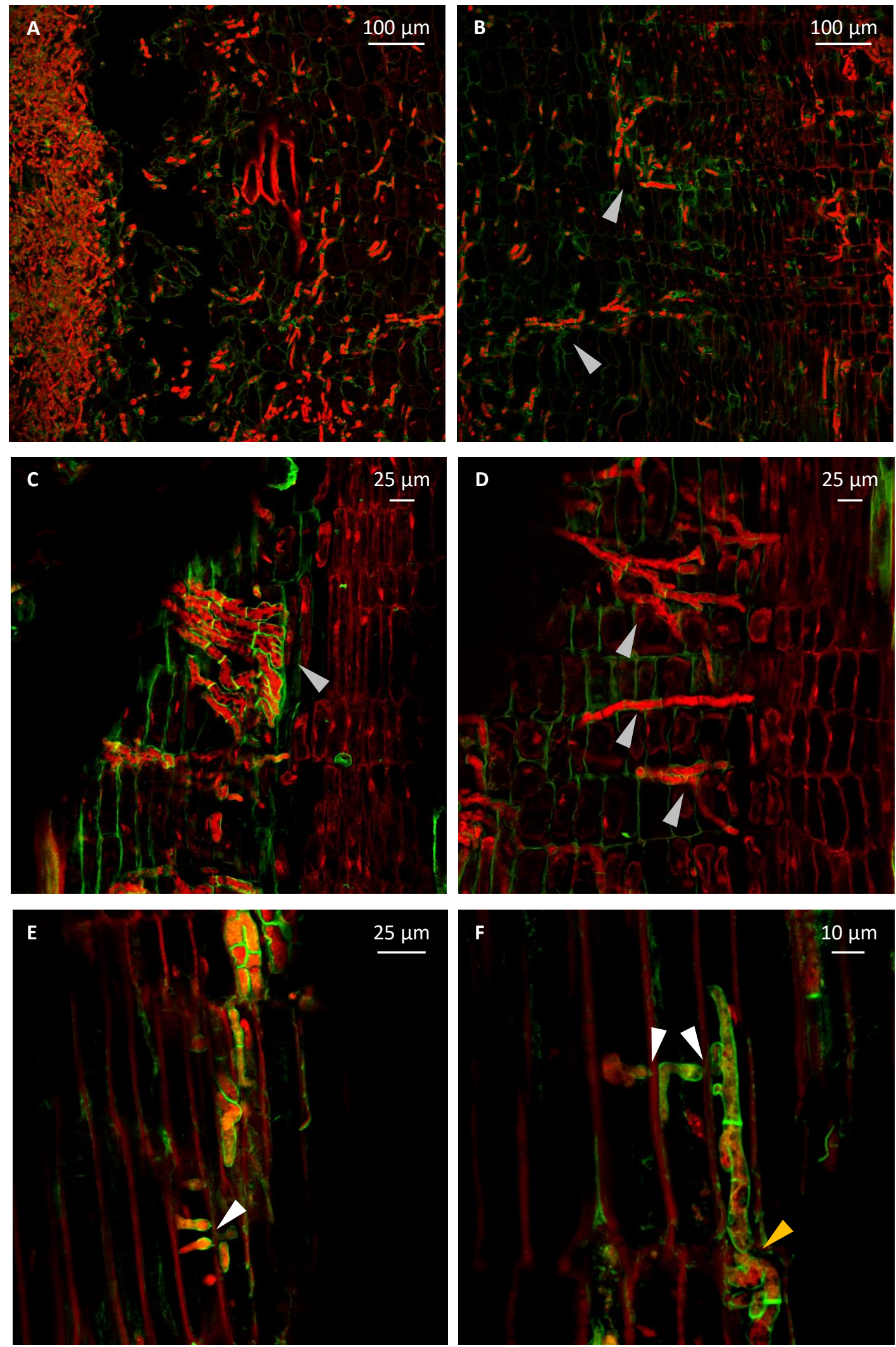

Fig. 26 CLSM imgages showing growth of S. sclerotiorum in the hypocotyl of oilseed rape (Loras), stained with Alexa Fluor and propidium iodide A: Mycelium mat (left) outside of the hypocotyl; 7 dpi B: Hyphae criss crossing within hypocotyl tissue, 7 dpi C: Front of older, vacuolated hyphae in the hypocotyl; 1 dpi D: Hyphal growth in cross direction to hypocotyl cells, showing intercellular advancement; 1 dpi E: Tapered hyphae thickening again after crossing the cell wall of xylem parenchyma (white arrow head), 3 dpi F: Hyphae forming a bundle before penetrating the cell wall 
of xylem parenchyma (yellow arrow head) and single hyphae traversing the cell wall (white arrow heads); 3 dpi . B-D: Hyphae are indicated with grey arrow heads.

In the hypocotyl of Zhongyou 821 (Fig. 27), the colonisation pattern was identical to Loras. Via the cortex, hyphae reached the stele then being colonised. Already in early infection stages, hyphae were growing parallel as well as crosswise to the plant axis (Fig. $27 \mathrm{~A}$ and B). The xylem parenchyma was colonised by crossing the cell wall accompanied by thickening of hyphae and formation of hyphal bundles (Fig. 27 C and D). No colonisation of the xylem was observed in both genotypes.
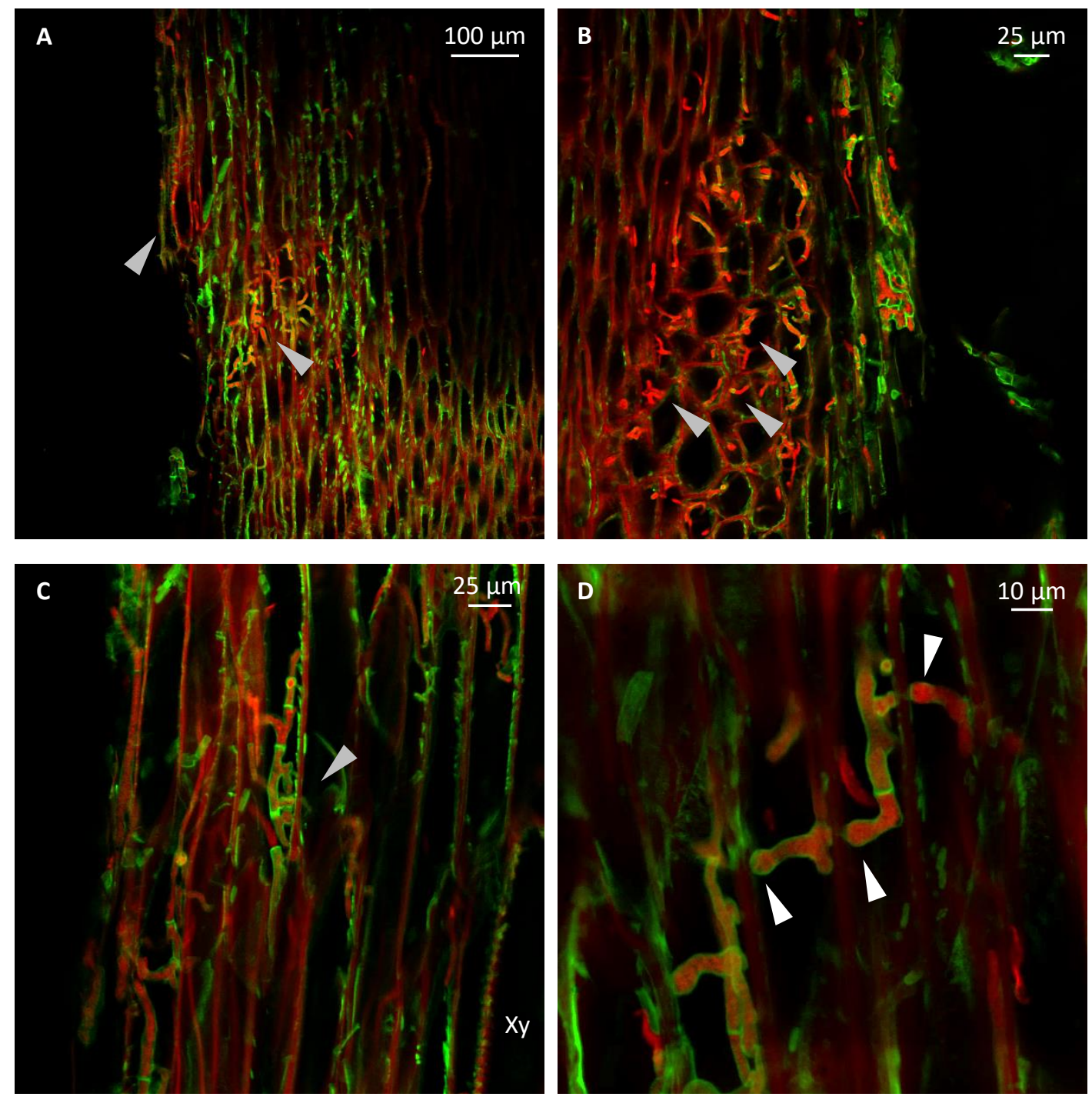

Fig. 27 CLSM imgages showing growth of S. sclerotiorum in the hypocotyl of oilseed rape (Zhongyou 821, $3 \mathrm{dpi}$ ), stained with Alexa Fluor and propidium iodide A: Hyphae are colonising the xylem parenchyma B: Ray parenchyma is colonised by hyphae C: Hyphae traversing the cell wall of xylem parenchyma D: Tapered hyphae thickening again after crossing the cell wall of xylem parenchyma (white arrow head). Hyphae are indicated with grey arrow heads; $X y-x y l e m$. 


\subsubsection{Histology of the root}

Longitudinal sections of the root show the different cell types including cortex, stele and ray parenchyma (Fig. 28). The different tissues like cortex and vascular bundles could be identified.

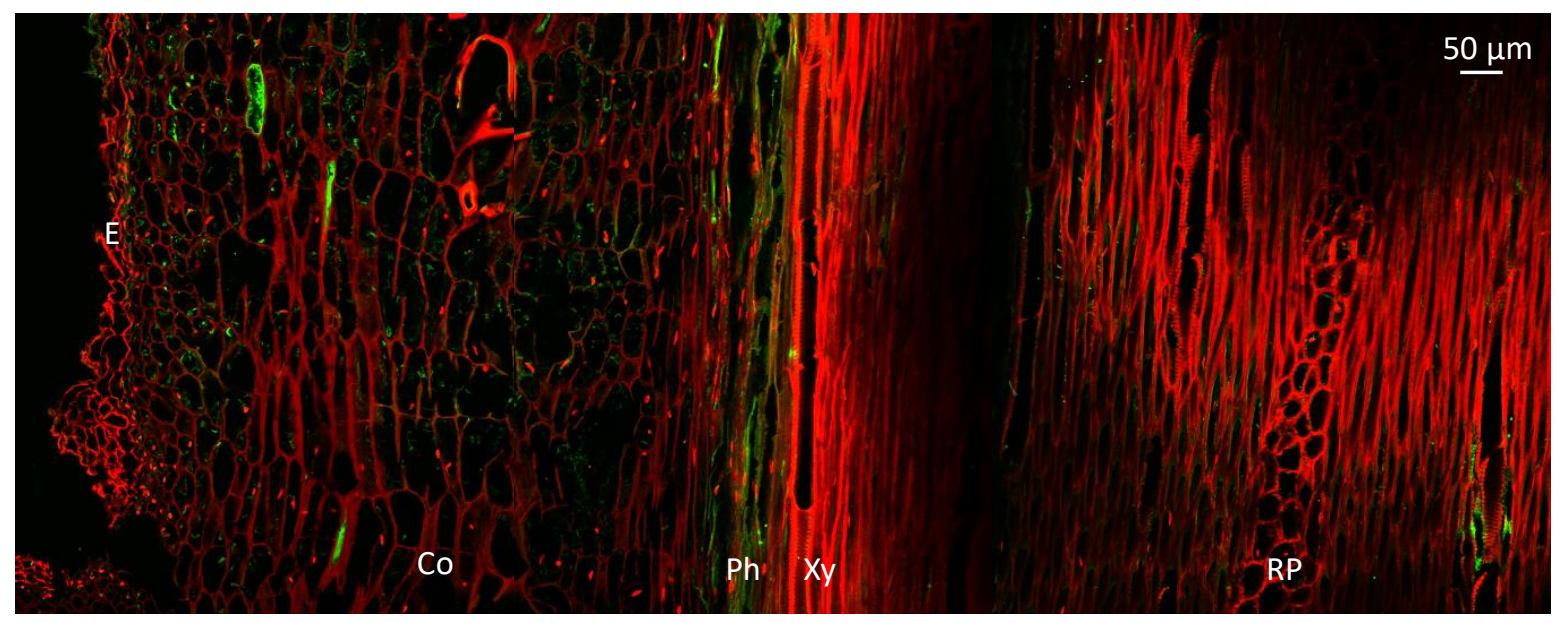

Fig. 28 CLSM imgage showing longitudinal section of non-inoculated oilseed rape root (Loras) after staining with Alexa Fluor and propidium iodide. E - epidermal layer, Co - cortex, Ph - phloem, Xyxylem, RP - ray parenchyma.

In the root of OSR, hyphae mainly grew in the cortex leading to a destruction of the root cells (Fig. 29 A and B). Unlike hyphal advancing in the stem, growth in the root appeared quite undirected without hyphae showing a directed pattern. Towards the stele, it was observed that hyphae tend to grow along the cells of ray parenchyma, only advancing into elongated cells of xylem parenchyma in rather individual cases (Fig. 29 C and D). 

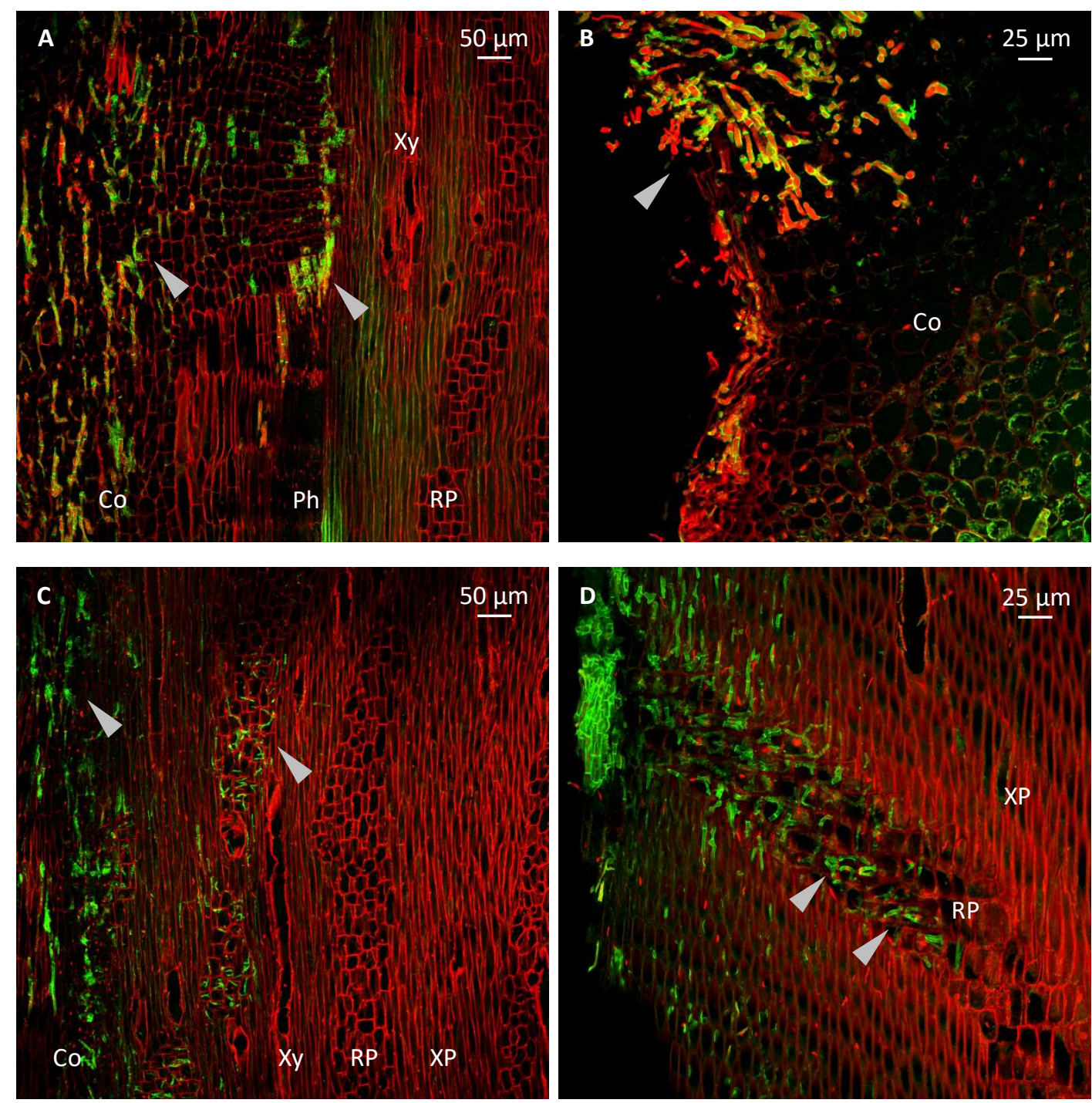

Fig. 29 CLSM images showing growth of S. sclerotiorum in the root of oilseed rape, stained with Alexa Fluor and propidium iodide A: Hyphae growing in the cortex and advancing into the phloem; Zhongyou 821, $7 \mathrm{dpi}$ B: Undirected hyphal growth in the cortex at exterior lesion edge; Zhongyou 821, 1 dpi C: Hyphae growing in the cortex and along the ray parenchyma; Zhongyou 821, $7 \mathrm{dpi}$ D: Hyphae growing along the ray parenchyma; Loras, $7 \mathrm{dpi}$. Hyphae are indicated with grey arrow heads. Co - cortex, Ph - phloem, Xy - xylem, RP - ray parenchyma, XP - xylem parenchyma.

As noted before, hyphae were found in the root cortex (Fig. $30 \mathrm{~A}$ ). When the plant tissue was already infected, hyphae still formed infection cushions at the plant surface above areas not yet being colonised. Hyphae advanced intercellularly (Fig. $30 \mathrm{~B}$ ), also when generated from the infection cushion (Fig. 30 C). Around the infection cushion, compact plant cells entangled the fungal penetration structures (Fig. $30 \mathrm{D}$ ). 

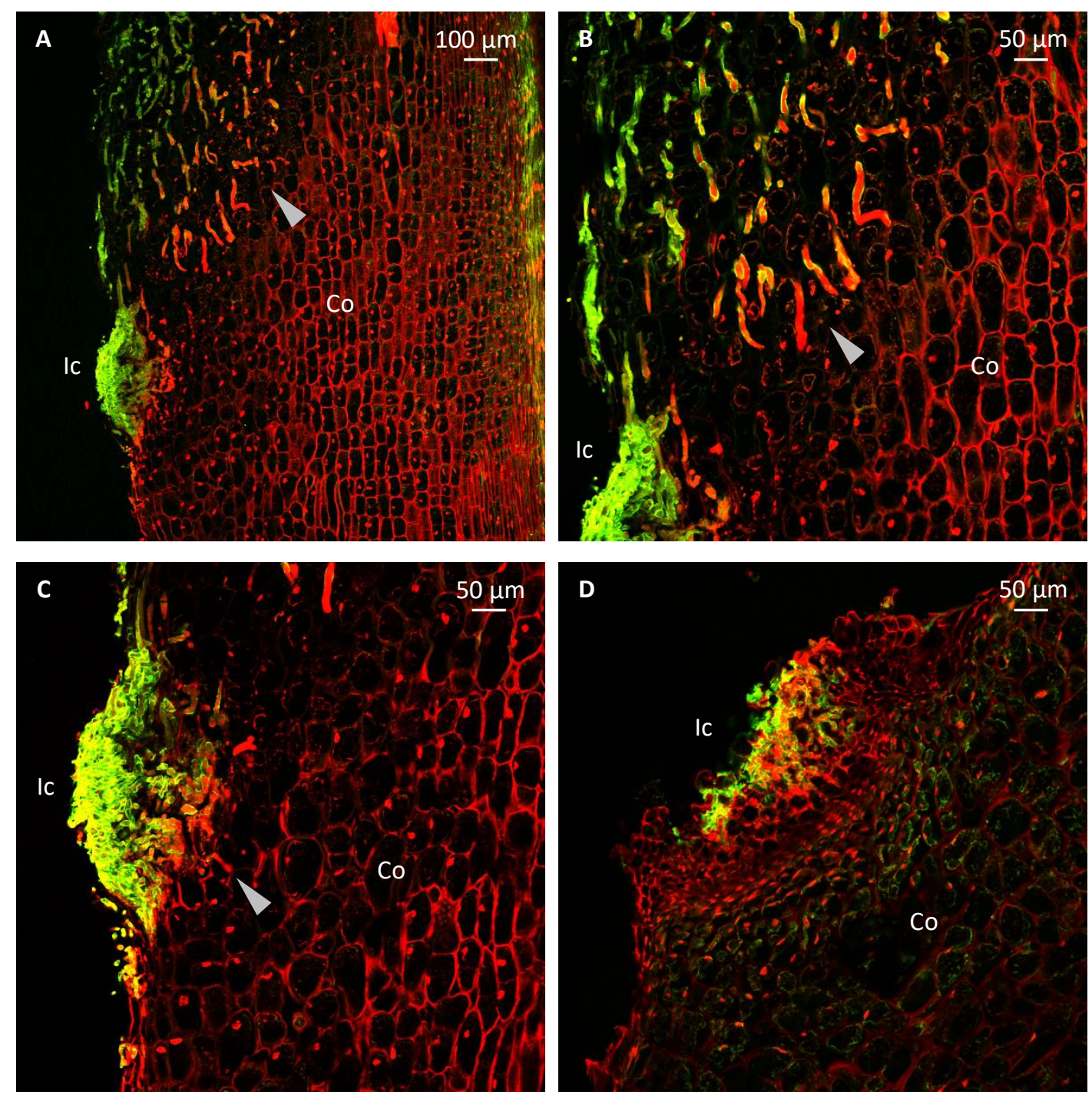

Fig. 30 CLSM images showing growth of S. sclerotiorum in root cortex of oilseed rape (Loras, $1 \mathrm{dpi}$ ), stained with Alexa Fluor and propidium iodide A: Undirected growth of hyphae and infection cushion B: Intercellularly advancing hyphae C: Infection cushion allows hyphae to penetrate into the cortex D: Plant cells shaping around the infection cushion. Hyphae are indicated with grey arrow heads. Co - cortex, Ic - infection cushion.

When hyphae advanced along the compact cells of ray parenchyma, they penetrated through the cell wall pits (Fig. $31 \mathrm{~A}$ and B). As seen before, they rarely 'escaped' along the longer cells of xylem parenchyma, but in single cases could also be found in the xylem (Fig.

$31 \mathrm{C})$. Fig. $31 \mathrm{D}$ gives a good impression on branched hyphae colonising the tissue and making optimal use of the cell wall pits. 

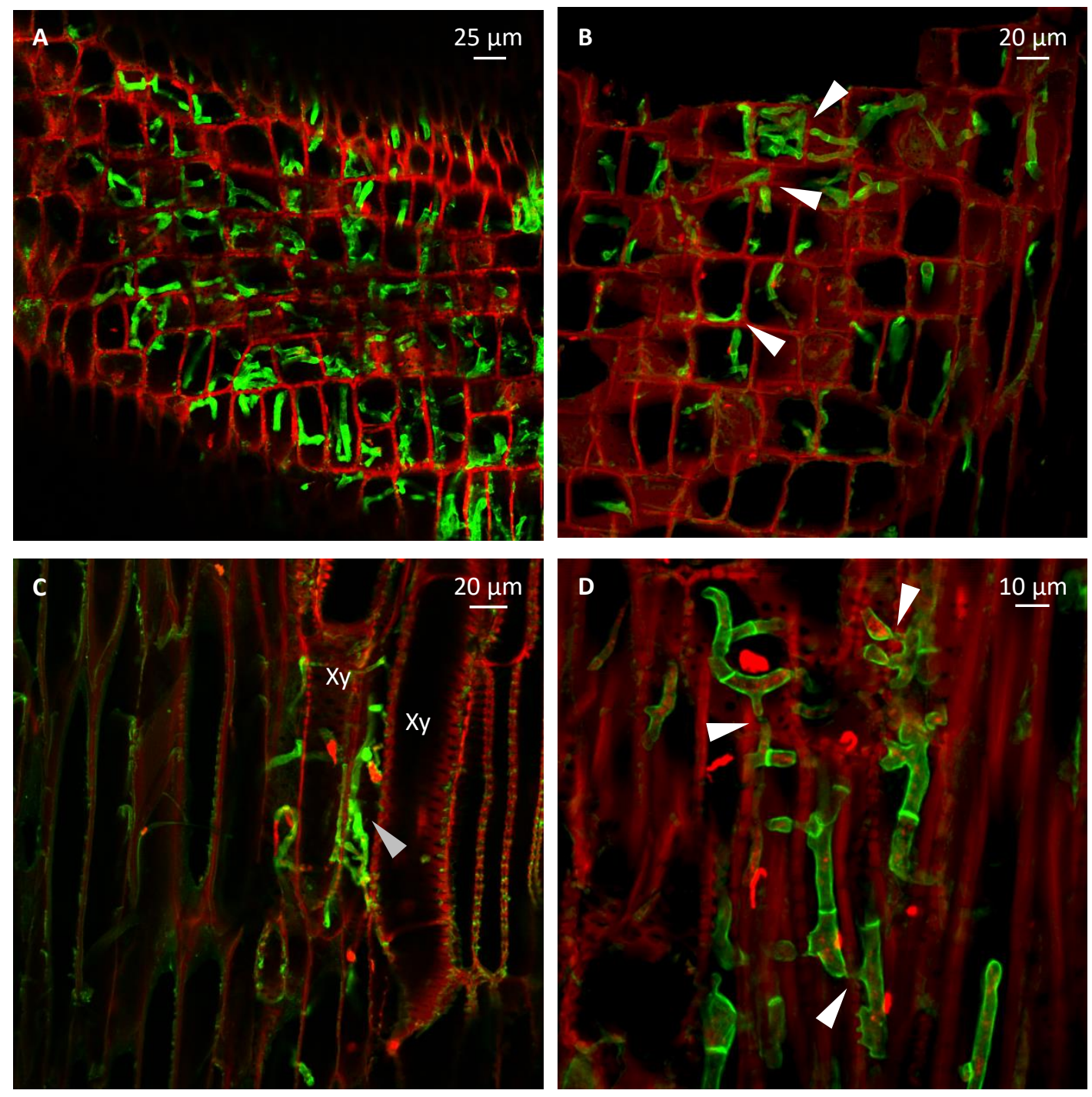

Fig. 31 CLSM images showing growth of S. sclerotiorum within stele of oilseed rape roots (7 dpi), stained with Alexa Fluor and propidium iodide A: Hyphae (green) growing along cells of ray parenchyma; Loras B: Hyphae forming bundles and using cell wall pits (white arrow heads) to advance; Zhongyou 821 C: Fungal hyphae (grey arrow head) within the xylem; Zhongyou 821 D: Hyphae traversing through cell wall pits (white arrow heads); Loras. $\mathrm{Xy}-\mathrm{xylem}$. 


\subsubsection{Histology of the leaf}

Infected leaves of Loras and Zhongyou 821 were stained with Chlorazol black E to visualise the hyphae of $S$. sclerotiorum. In the microscope, infection cushions were visible from which the fungal mycelium spread over the leaf surface (Fig. 32). Several infection cushions were located in one spot (Fig. 32 A), and hyphae spread out around them (Fig. 32 B). Within the plant tissue only few fungal structures were visible. There were no differences between the genotypes.
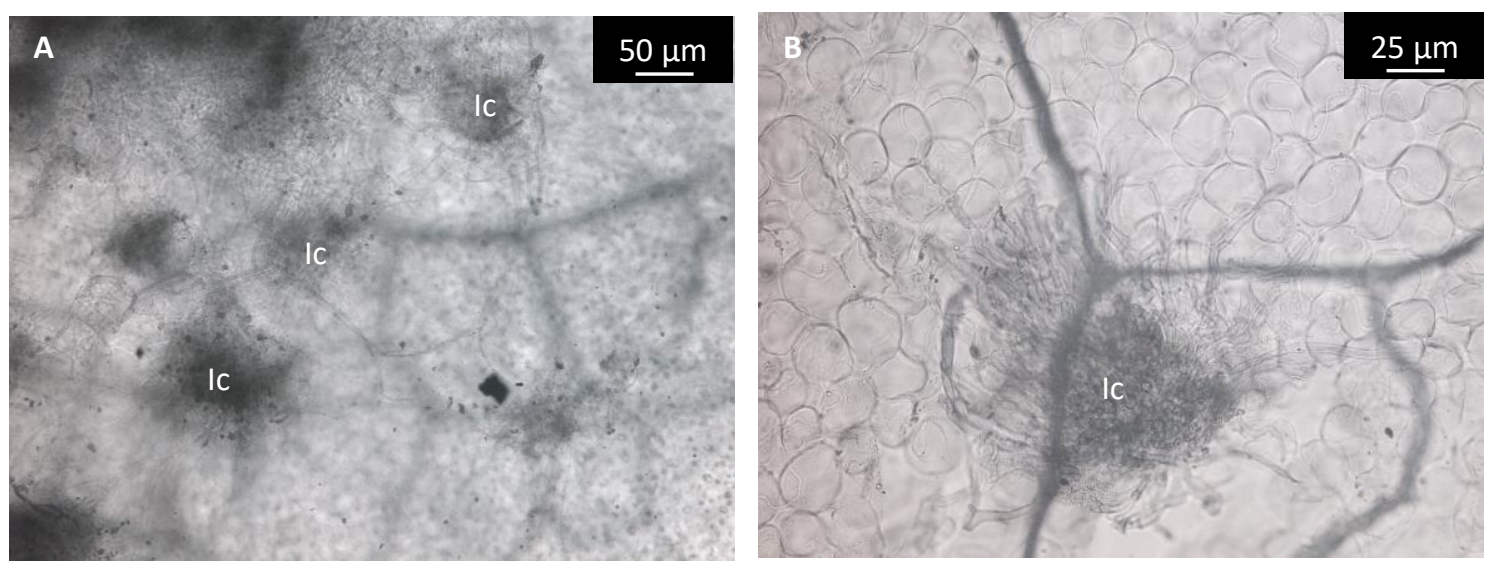

Fig. 32 Light microscopy image of oilseed rape leaves infected with S. sclerotiorum, stained with Chlorazol black E A: Several infection cushions on the leaf surface; Loras, 72 hpi B: Single infection cushion with superficially radiating hyphae; Zhongyou $821,48 \mathrm{hpi}$. Ic - infection cushion.

Observation of the leaf surface showed that hyphae advanced in a fan but also were forming fronts (Fig. 33).
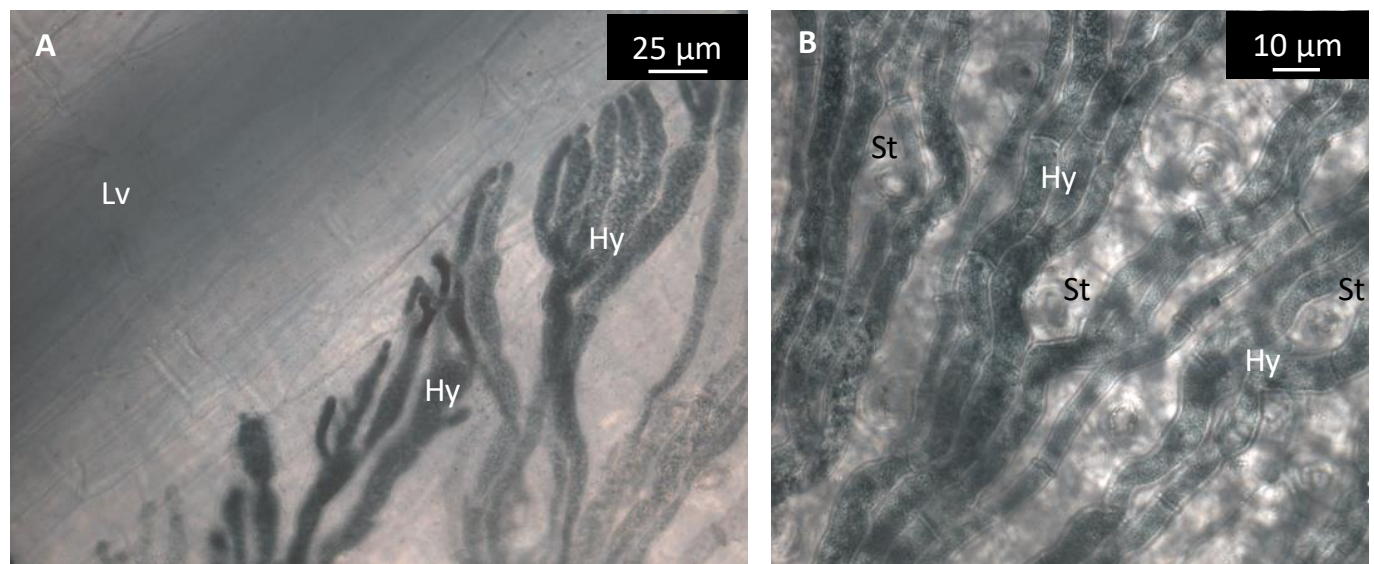

Fig. 33 Light microscopy image of oilseed rape leaves infected with S. sclerotiorum, stained with Chlorazol black E A: Growing hyphae at the leaf surface form front near a leaf vein; Zhongyou 821, $72 \mathrm{hpi} \mathrm{B:} \mathrm{Superficially} \mathrm{growing} \mathrm{hyphae} \mathrm{shape} \mathrm{around} \mathrm{stomata;} \mathrm{Loras,} 72 \mathrm{hpi}$. Hy - hyphae, Lv - leaf vein, St - stoma. 
When hyphae encountered elevated structures like leaf veins or stomata, they overgrew them after gathering several hyphae together (Fig. 33 A) or simply grew around them (Fig. $33 \mathrm{~B})$.

Chlorazol black stain was more intense on the hyphal tips where plant cells are more compact, while the older parts of each hyphae appeared less stained (Fig. 34 A). On the leaf surface, hyphae branched and grew across leaf veins or along them. At the same region but inside the leaf tissue, there were no fungal structures present (Fig. 34 B). Vascular bundles, especially the xylem, remained clear (Fig. 34 B and C). When single hyphae were detected within the leaf, they usually grew along - but not inside - the vascular bundles, even following them when branching (Fig. 34 D).
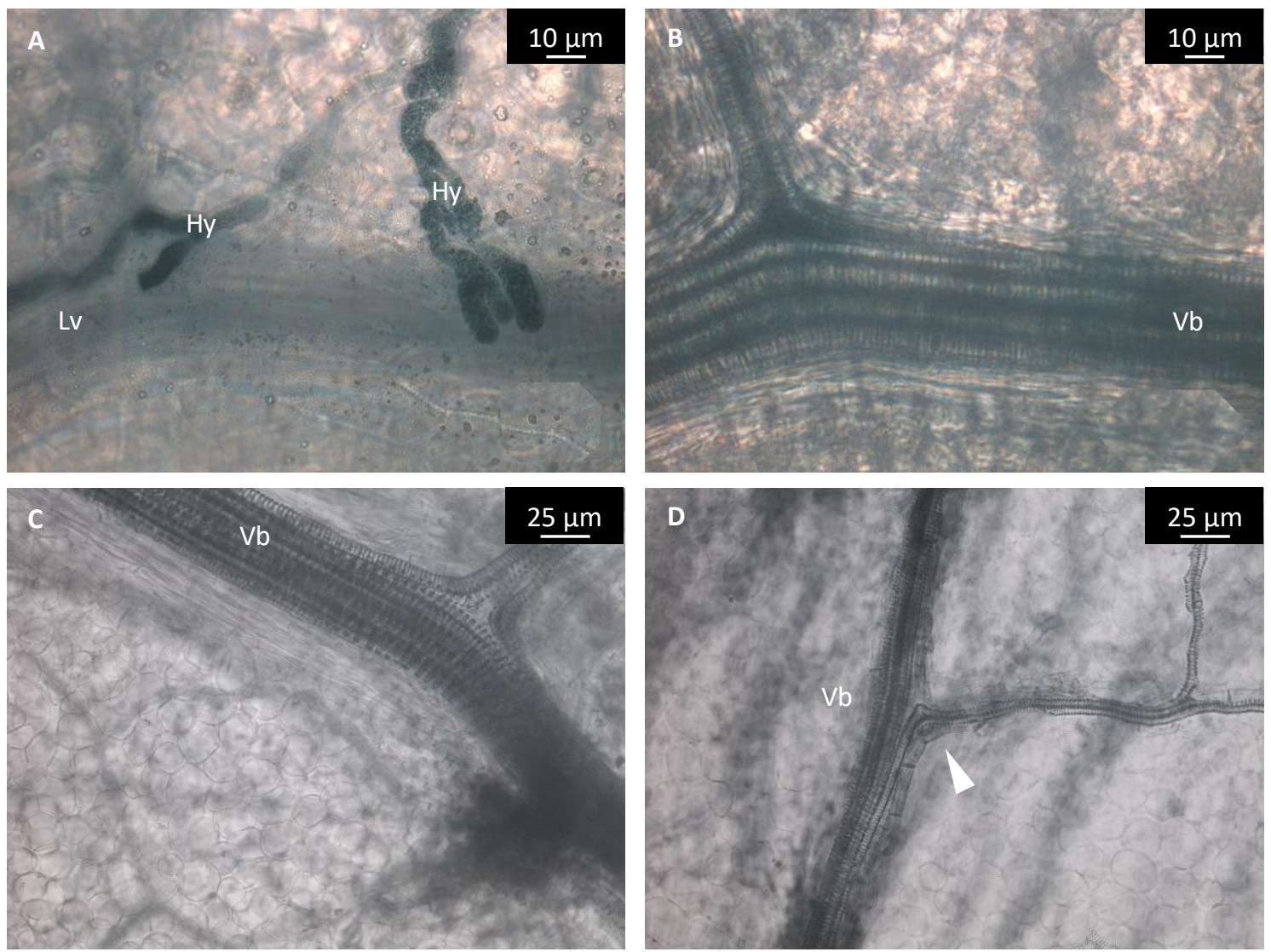

Fig. 34 Light microscopy image of oilseed rape leaves infected with S. sclerotiorum, stained with Chlorazol black A: Surepidermal hyphae growing over an elevated leaf vein; Loras, 48 hpi B: No hyphae are within the tissue in the same area; Loras, 48 hpi C: Vascular bundles remain clear of hyphae; Loras, 72 hpi D: Hyphae growing along vascular bundle (white arrow head) but not in vessels; Zhongyou 821, 48 hpi. Hy - hyphae, Lv - leaf vein, Vb - vascular bundle. 


\subsubsection{Special investigations on tyloses}

Tyloses were formed in both genotypes at infection stages later than $1 \mathrm{dpi}$. Non-infected plant tissue did not contain tyloses. Furthermore, after infection they could be found in all plant parts, namely root (Fig. 35), hypocotyl (Fig. 36) and stem (Fig. 37). They were only present in xylem cells being located near the fungal hyphae, and occurred only in the infection stages later than $1 \mathrm{dpi}$. Tyloses showed a different emission wavelength of bright red (Fig. $35 \mathrm{~A}$ and B) or faintly green (Fig. $35 \mathrm{C}$ and D).
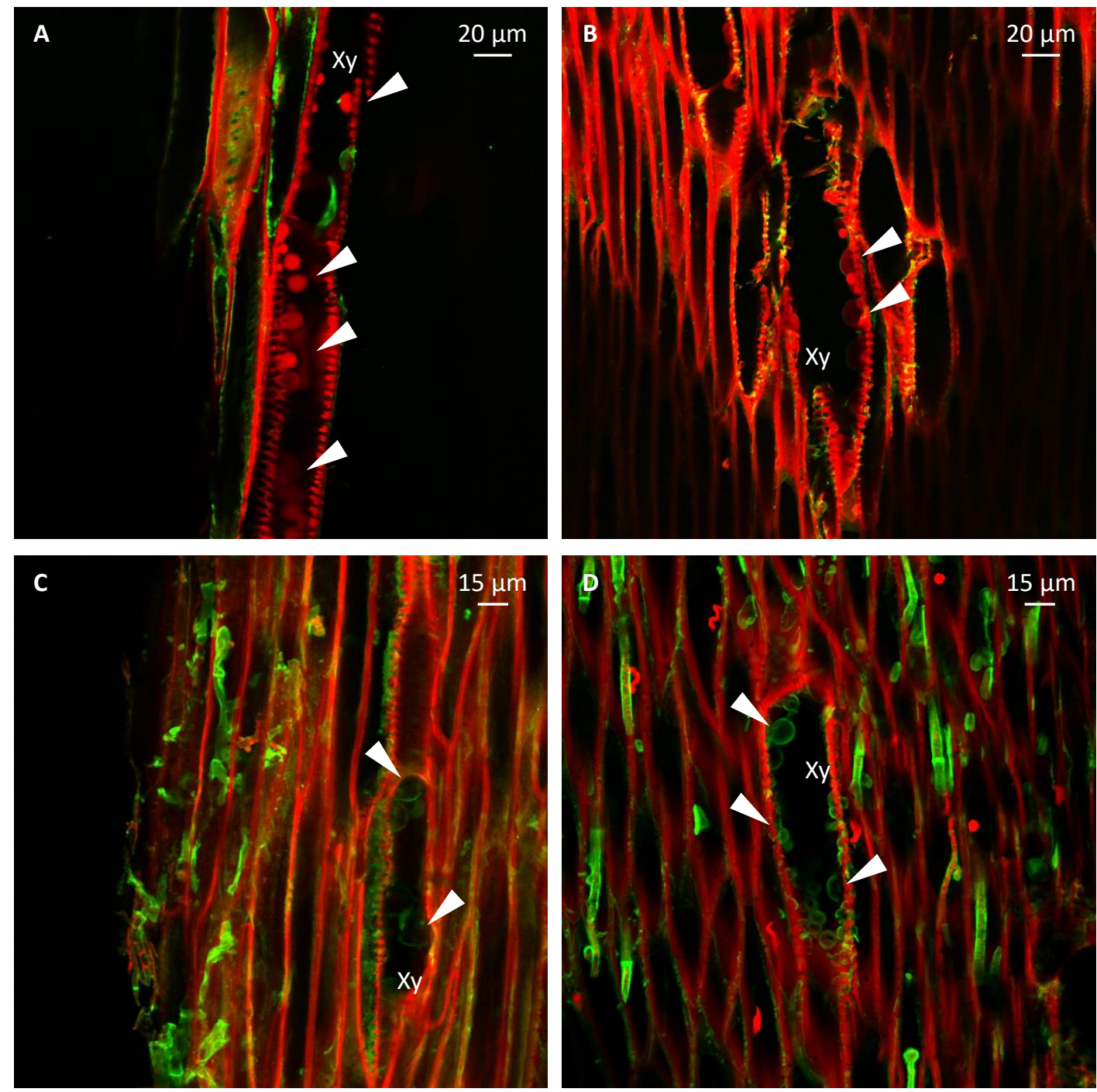

Fig. 35 CLSM images showing different shapes of tyloses in the xylem of oilseed rape roots infected by S. sclerotiorum (7 dpi), stained with Alexa Fluor and propidium iodide $\mathbf{A}$ and B: Bright red light emitting tyloses; Loras C: Faintly green shining tyloses; Zhongyou 821 D: Faintly green shining tyloses; Loras. Xy-xylem.

These different types of xylem vessel blocking structures were further investigated with different staining methods: 
Toluidine blue was used to stain tyloses and identify their composition (Fig. 36). The dye stained primary cell wall material coating pectic substances purple (e.g. epidermis, cortex parenchyma, sclerenchyma fibres, phloem, pith, tyloses), and stained lignin containing and secondary cell wall material blue (e.g. xylem, xylem parenchyma). This method confirmed what has been observed and described before.

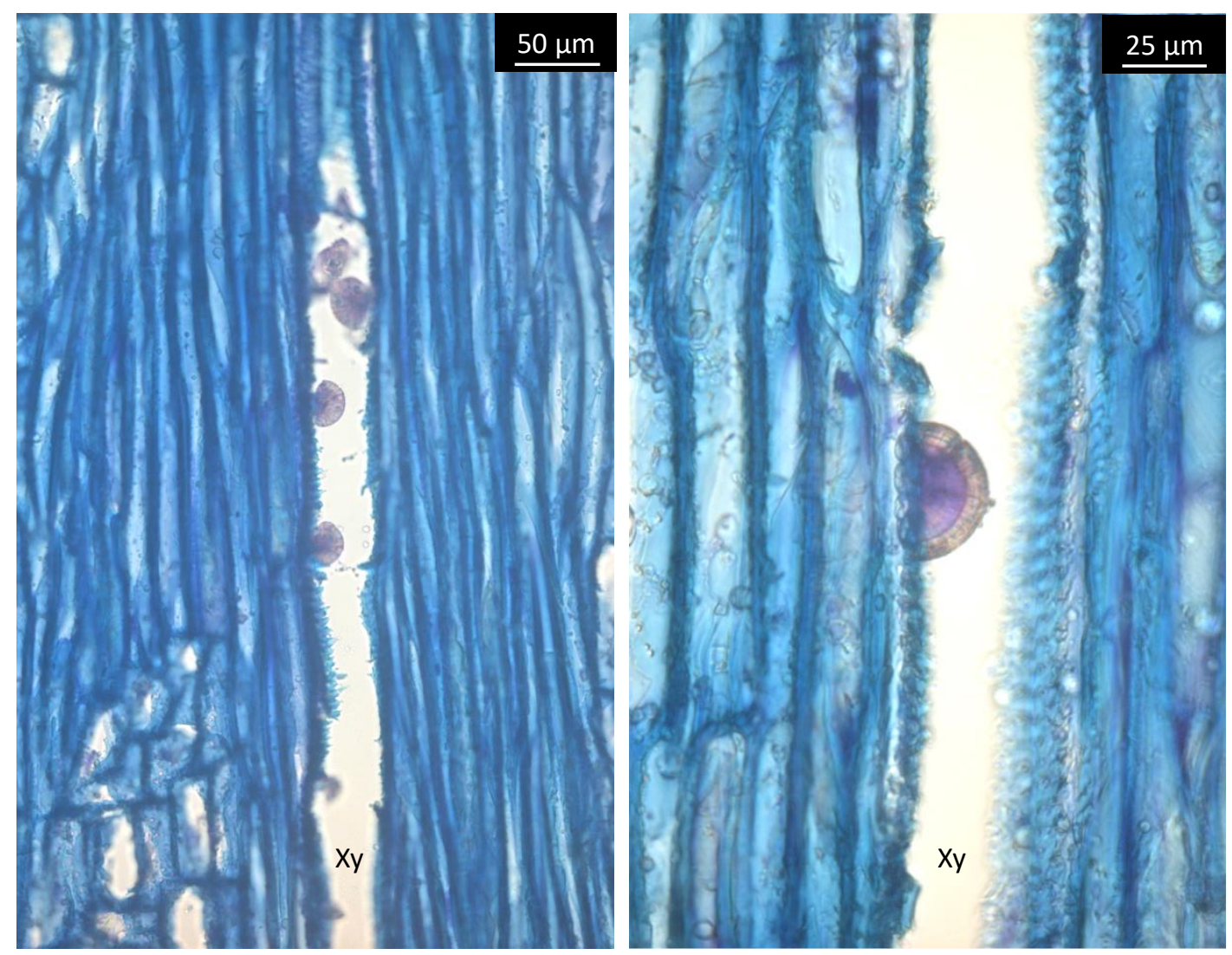

Fig. 36 Light microscopy images of longitudinal section of oilseed rape hypocotyl (Loras) after infection with $S$. sclerotiorum ( $7 \mathrm{dpi}$ ), stained with toluidine blue showing tyloses in purple located within the xylem; $x y-x y l e m$.

Trypan-aniline blue double stain was applied to study callose deposition. However, callose deposition was not visible in the sections (Fig. 37). These sections gave another example for tyloses appearing in different genotypes and plant parts since they show tyloses in the xylem of an infected stem of Zhongyou 821 (Fig. $37 \mathrm{~A}$ ) and in the hypocotyl of Loras (Fig. 37 C), both at $3 \mathrm{dpi}$. Fig. $37 \mathrm{~B}$ shows that tyloses emitted a faint autofluorescence. There was no difference in the number of tyloses formed in the different genotypes or plant parts. 

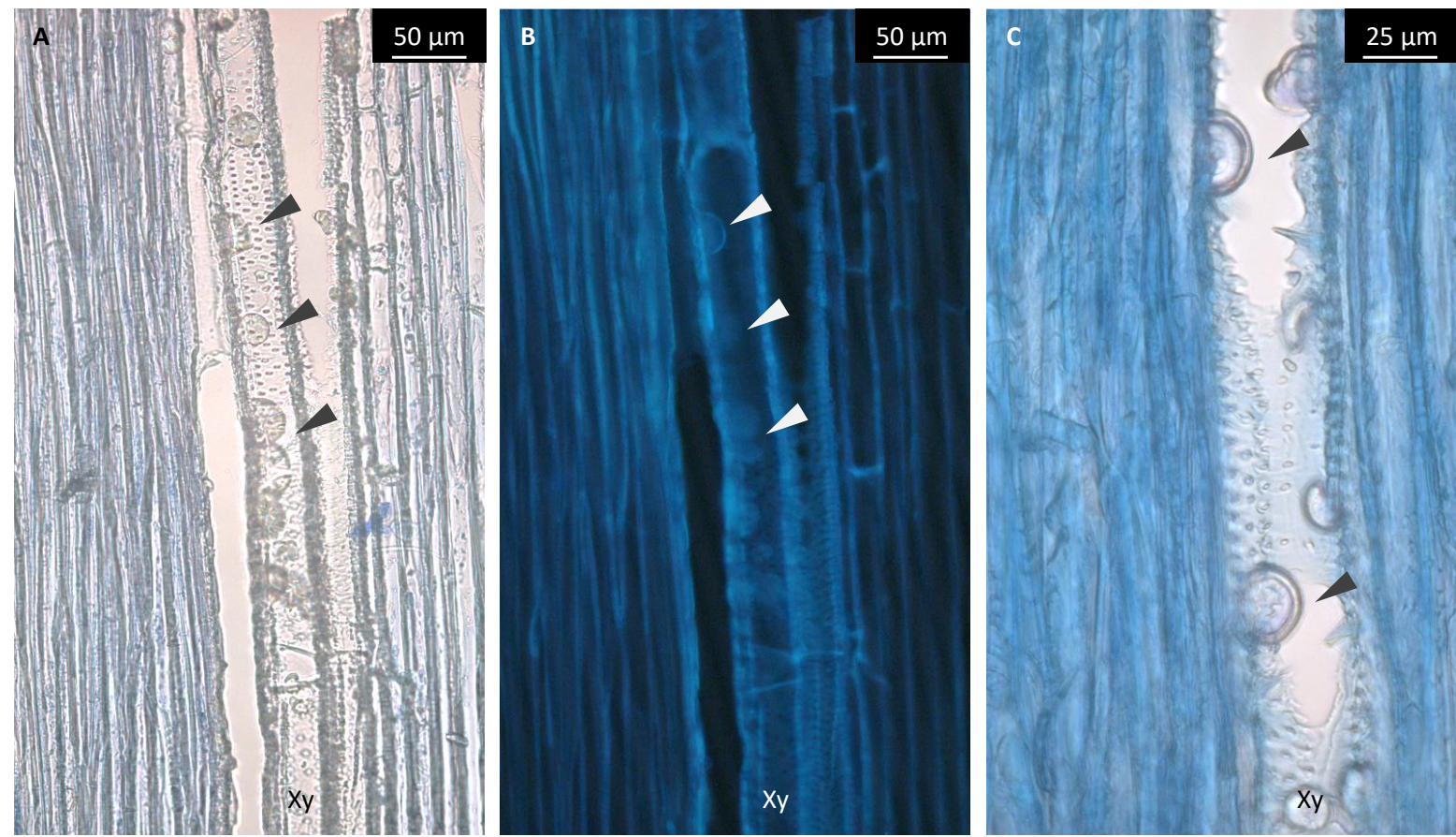

Fig. 37 Microscopy images of longitudinal sections of oilseed rape 3 days after infection with S. sclerotiorum, stained with trypan-aniline blue double stain showing tyloses in xylem vessels A: Light image of tyloses in the stem of Zhongyou 821 B: Fluorescence emission of the same zone C: Light microscopy image of tyloses in the hypocotyl of Loras. Tyloses are indicated with arrow heads. Xy-xylem.

Periodic acid Schiff's reagent did not stain tyloses as intended. Plant tissue and fungal hyphae were equally stained in pink. Observation under the light microscope did not lead to identification of tyloses.

\subsection{Biochemical responses to infection}

Effects of oilseed rape genotypes (Loras and Zhongyou 821), treatment (control and inoculated with S. sclerotiorum) and plant part (stem, hypocotyl and/or root) were studied with regard on phenolic compounds, peroxidase activity and glucosinolate content.

\subsubsection{Phenolic compounds}

Phenolic compounds were studied in stem and hypocotyl of oilseed rape plants. Seven phenolic acids representing commonly approved key substances have been identified and quantified via HPLC-analysis: salicylic acid, $t$-cinnamic acid, $p$-coumaric acid, chlorogenic acid, caffeic acid, ferulic acid and sinapic acid. Free and conjugated forms could be analysed 
for all but salicylic acid, which has been only extracted in the free form. Effect of genotype, inoculation and plant tissue were investigated.

Inoculation of stem and hypocotyl of oilseed rape had different effects on the conjugated and free phenolic acids. There was no significant effect of inoculation on conjugated phenolics and only tendencies could be observed (Fig. 38). In the conjugated form only $t$ cinnamic increased slightly after infection. The inoculation had a contrary effect on conjugated caffeic acid and sinapic acid located in the hypocotyl, resulting in a decrease. The free form of $t$-cinnamic acid, caffeic acid and chlorogenic acid showed an increase after infection. The effect of inoculation was statistically highly significant in free $t$-cinnamic, caffeic and chlorogenic acid (Kruskal-Wallis ANOVA, $P<0.01$ ). There was no genotypic effect.
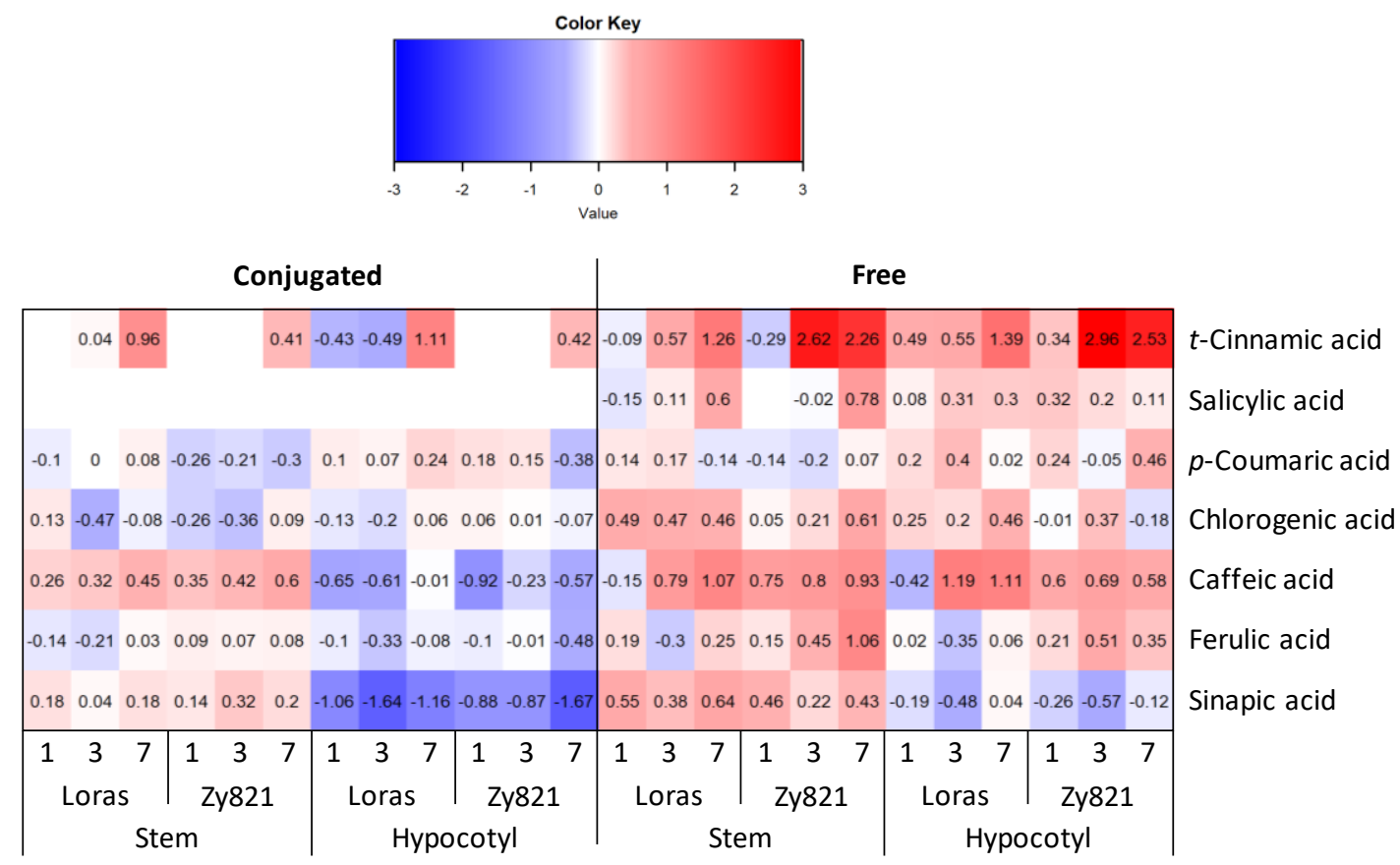

Fig. 38 Effect of inoculation with S. sclerotiorum on conjugated and free phenolic acids in stem and hypocotyl of the oilseed rape genotypes Loras and Zhongyou 821 at 1, 3 and $7 \mathrm{dpi}$.

With respect to the plant tissue effect it can be said that compared to the hypocotyl, the stem generally tended to contain a significantly higher amount of conjugated caffeic acid, ferulic acid and sinapic acid (Fig. 39; Kruskal-Wallis ANOVA, $P<0.05$ ). The free form of these three phenolic acids showed a similar tendency. Amounts of other compounds like both conjugated $p$-coumaric and chlorogenic acid, and free $p$-coumaric acid were significantly lower in the stem compared to the hypocotyl (Kruskal-Wallis ANOVA, $P<0.05$ ). 


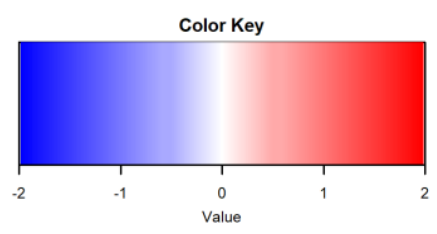

\begin{tabular}{|c|c|c|c|c|c|c|c|c|c|c|c|c|c|c|c|c|c|c|c|c|c|c|c|c|}
\hline \multicolumn{12}{|c|}{ Conjugated } & \multicolumn{12}{|c|}{ Free } & \multirow[b]{2}{*}{$t$-Cinnamic acid } \\
\hline 1.29 & & & -0.17 & & -0.16 & & 0.53 & -0.15 & & & -0.01 & -0.06 & -0.37 & -0.21 & & & & -0.58 & 0.02 & -0.13 & -0.63 & -0.34 & -0.27 & \\
\hline & & & & & & & & & & & & 0.1 & -0.55 & -0.39 & -1.36 & -0.92 & -0.44 & -0.22 & -0.21 & 0.3 & & -0.22 & 0.67 & Salicylic acid \\
\hline-0.25 & -0.46 & -0.28 & -0.58 & -0.16 & -0.28 & -0.2 & -0.08 & -0.15 & -0.44 & -0.36 & 0.08 & -0.27 & -0.43 & -0.16 & -0.21 & -0.32 & 0.08 & -0.05 & -0.23 & -0.16 & -0.38 & -0.15 & -0.39 & $p$-Coumaric acid \\
\hline-0.02 & -0.14 & -0.11 & -0.12 & -0.07 & -0.26 & 0.26 & -0.27 & -0.14 & -0.32 & -0.37 & 0.16 & -0.03 & -0.36 & 0.11 & 0.02 & 0.2 & 0.31 & 0.24 & 0.27 & 0.01 & 0.06 & -0.16 & 0.79 & Chlorogenic acid \\
\hline 1.17 & 1.03 & 1.1 & 0.96 & 0.72 & 1.48 & 0.91 & 0.93 & 0.45 & 1.27 & 0.65 & 1.17 & 0.39 & -0.44 & -0.08 & 0.47 & -0.2 & 0.82 & 0.28 & -0.4 & -0.04 & 0.15 & 0.11 & 0.35 & Caffeic acid \\
\hline 0.15 & 0.07 & 0.04 & -0.09 & 0.14 & -0.01 & -0.05 & 50.12 & 0.11 & 0.19 & 0.07 & 0.56 & 0.14 & 0.6 & 0.36 & -0.2 & 0.01 & 0.06 & 0.17 & 0.05 & 0.19 & -0.05 & -0.06 & 0.71 & Ferulic acid \\
\hline 1.71 & 1.49 & 1.64 & 0.97 & 1.21 & 0.97 & 1.23 & 1.68 & 1.35 & 1.02 & 1.19 & 1.87 & 0.85 & 0.5 & 0.3 & 0.06 & 0.46 & 0.14 & 0.74 & 0.86 & 0.6 & 0.72 & 0.79 & 0.55 & Sinapic acid \\
\hline 1 & $\begin{array}{c}3 \\
\text { Loras }\end{array}$ & $\begin{array}{r}7 \\
\text { s }\end{array}$ & ${ }^{1}$ & $\begin{array}{c}3 \\
y 821\end{array}$ & $\begin{array}{r}7 \\
1\end{array}$ & & $\begin{array}{c}3 \\
\text { Lora }\end{array}$ & $\begin{array}{r}7 \\
s\end{array}$ & ${ }^{1}$ & $\begin{array}{r}3 \\
-2 y 82\end{array}$ & 1 & 1 & $\begin{array}{c}3 \\
\text { Loras }\end{array}$ & $\begin{array}{r}7 \\
\text { Is }\end{array}$ & & $\begin{array}{c}3 \\
Z y 82\end{array}$ & 7 & & $\begin{array}{c}3 \\
\text { Lora }\end{array}$ & 7 & & $\begin{array}{c}3 \\
-2 y 821\end{array}$ & $\begin{array}{l}7 \\
1\end{array}$ & \\
\hline & & Cont & trol & & & & & nocl & ulate & & & & & Cor & ntro & & & & & nocu & ulate & & & \\
\hline
\end{tabular}

Fig. 39 Effect of plant part (stem related to hypocotyl) on conjugated and free phenolic acids in oilseed rape genotypes Loras and Zhongyou 821 non-inoculated and inoculated at 1, 3 and $7 \mathrm{dpi}$ with S. sclerotiorum.

In total, the conjugated form of the phenolic acids had higher amounts than the free form (Fig. 40). Both $p$-coumaric acid and ferulic acid were higher in the conjugated form. Caffeic acid and sinapic acid in the stem were higher in the conjugated form, but in the hypocotyl were higher in the free form. Free chlorogenic acid also was higher in hypocotyl. Raw data can be found in the annex (Tab. 7A and Tab. 8A). 


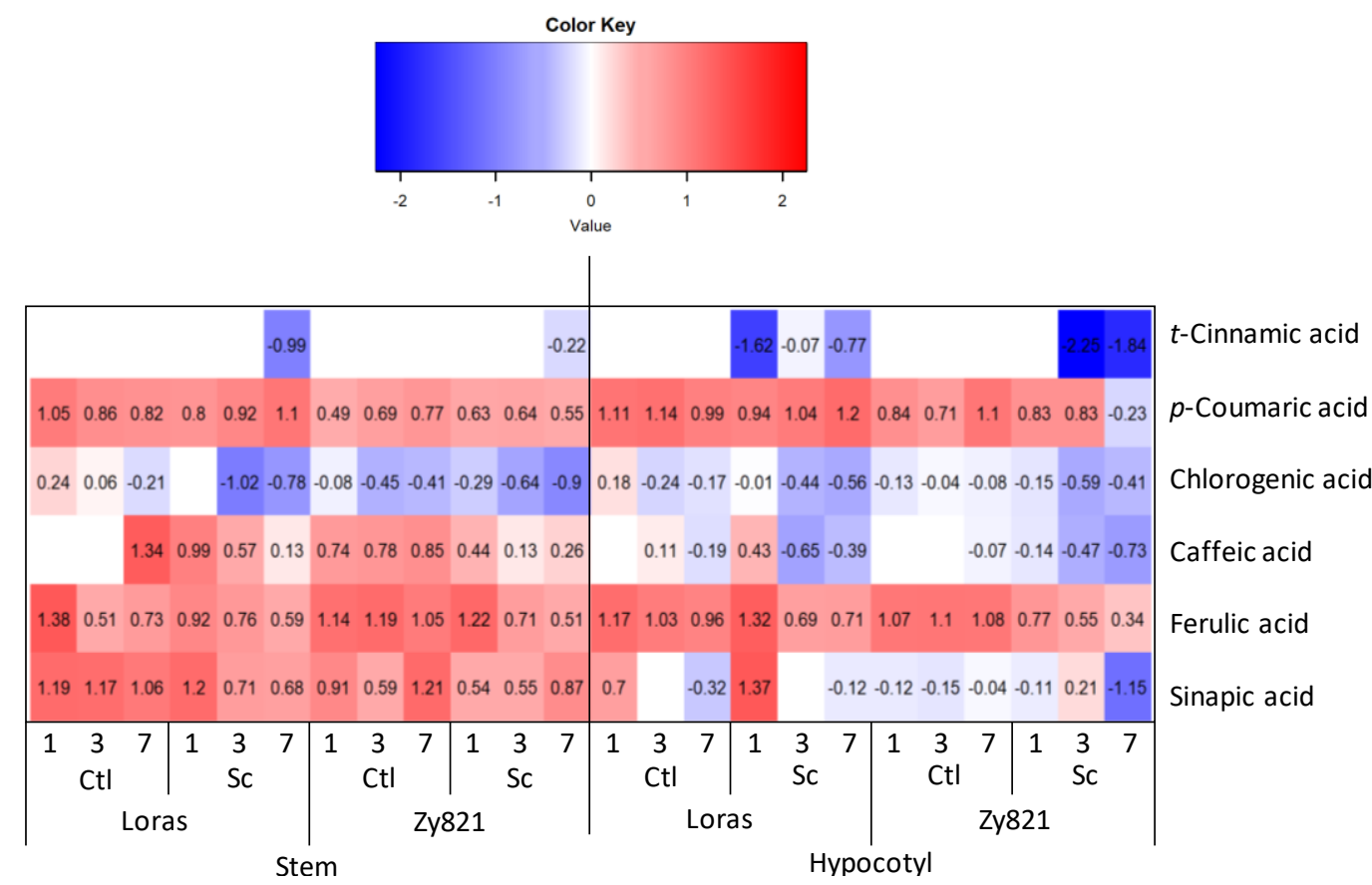

Fig. 40 Relation of conjugated to free phenolic acids in stem and hypocotyl of the oilseed rape genotypes Loras and Zhongyou 821 in non-inoculated plants and at 1, 3 and 7 days post inoculation with S. sclerotiorum.

\subsubsection{Peroxidase activity}

Peroxidase (POX) activity was analysed via photometer in samples of three experiments on oilseed rape inoculated with S. sclerotiorum. One experiment targeted differentiation between the plant tissues stem and hypocotyl including a repetition, and another experiment was directed at comparing stem and root of oilseed rape. To show the effect of inoculation on peroxidase, the POX ratio of optical density between inoculated and control plants was calculated. A ratio of 1 means that inoculation does not influence POX activity. A ratio bigger than 1 describes increasing POX activity, and a ratio lower than 1 describes decreasing POX activity due to inoculation of the plant tissues with S. sclerotiorum.

Peroxidase activity was significantly higher in the hypocotyl of Loras due to the genotypic effect (Fig. 41, see also annex Tab. 9A). No significant effect of inoculation was found on the activity of POX in the stem of Loras and in the stem and hypocotyl of Zhongyou 821. These results could be confirmed in the repetition of the experiment insofar as experimental factors had no significant effect on POX activity (see annex Tab. 10A). 


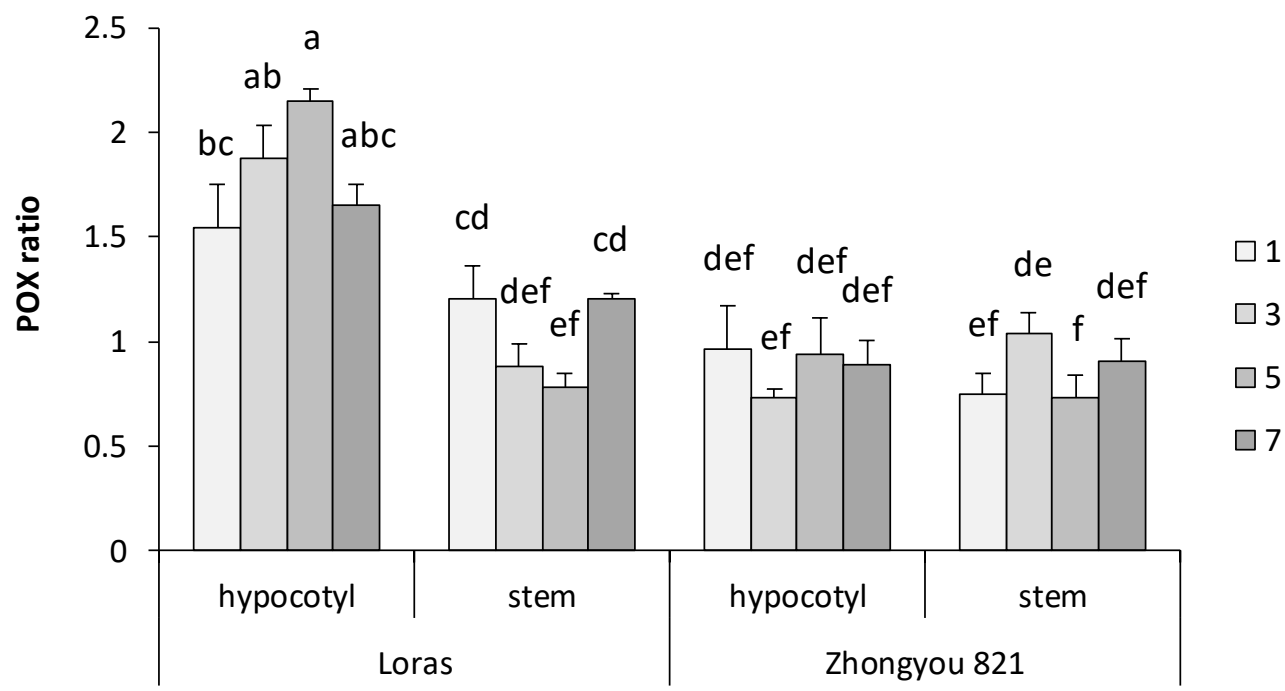

Fig. 41 Ratio of peroxidase activity between control and inoculated plants in stem and hypocotyl of the oilseed rape genotypes Loras and Zhongyou 821 at 1, 3, 5 and $7 \mathrm{dpi}$ with S. sclerotiorum. Mean values and standard errors are shown. Letters indicate significant differences (LSD test: $n=3, P<0.05$ ).

The genotype had a significant effect on POX activity (Tab. 16). However, the plant part did not significantly affect POX activity.

Tab. 16 F-values of main effect ANOVA of genotype, plant tissue and the interaction on ratio of the peroxidase activity including the degrees of freedom (df). Asterisks represent differences according to significance level (ANOVA: $n=3 ;{ }^{* *} p<0.001,{ }^{* *} p<0.01,{ }^{*} p<0.05$ and ns not significant).

\begin{tabular}{lll}
\hline Effect & df & POX ratio \\
\hline Genotype & 1 & $19.55^{* * *}$ \\
Plant tissue & 1 & $1.69^{\text {ns }}$ \\
Genotype $\times$ Plant tissue & 1 & $2.67^{\text {ns }}$ \\
\hline
\end{tabular}

In another experiment, the inoculation had a slight effect on POX activity in the roots causing an increase over time (Fig. 42, see also annex Tab. 11A). Results were more stable in the stem where no effect was observed. The POX activity was slightly but not significantly lower in the root than in the stem of Zhongyou 821. 


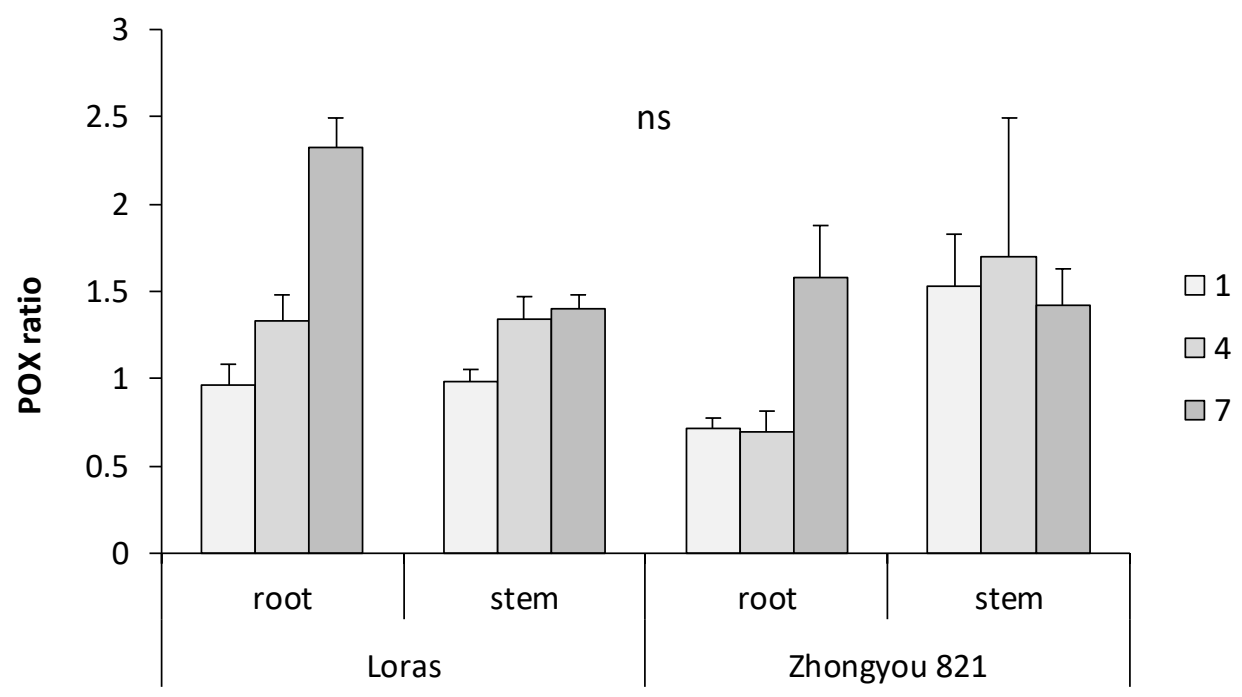

Fig. 42 Ratio of peroxidase activity between control and inoculated plants in stem and root of the oilseed rape genotypes Loras and Zhongyou 821 at 1, 4 and $7 \mathrm{dpi}$ with S. sclerotiorum. Mean values and standard errors are shown. Differences were not significant (Kruskal-Wallis ANOVA: $n=3$, $P<0.05)$.

However, differences caused by main effects genotype, plant tissue or the interaction were not statistically significant (Tab. 17).

Tab. 17 F-values of main effect ANOVA of genotype, plant tissue and the interaction on ratio of peroxidase activity including the degrees of freedom (df). Asterisks represent differences according to significance level (ANOVA: $n=3 ; * * *<<0.001, * * p<0.01,{ }^{*} p<0.05$ and ns not significant).

\begin{tabular}{lll}
\hline Effect & df & POX ratio \\
\hline Genotype & 1 & $1.85^{\mathrm{ns}}$ \\
Plant tissue & 1 & $3.04^{\mathrm{ns}}$ \\
Genotype $\times$ Plant tissue & 1 & $1.66^{\mathrm{ns}}$ \\
\hline
\end{tabular}

POX activity was not correlated with inoculation with S. sclerotiorum in any of the experiments.

\subsubsection{Glucosinolates}

\section{Glucosinolate pattern in different plant tissues}

Effects of inoculation, genotype and plant tissue on the glucosinolates (GSL) were investigated. Two experiments were conducted to investigate 1) GSL in the stem and hypocotyl and 2) GSL in the stem and the root. Both experiments (1 and 3) and the repetitions ( 2 and 4 ) showed no effect of the day post inoculation. In total, GSL content in 
the stem was measured in each experiment and hence was analysed four times. The very first experiment (1) showed a total stem GSL amount lower than in the following three (Fig. 43), with even significant differences compared to them.

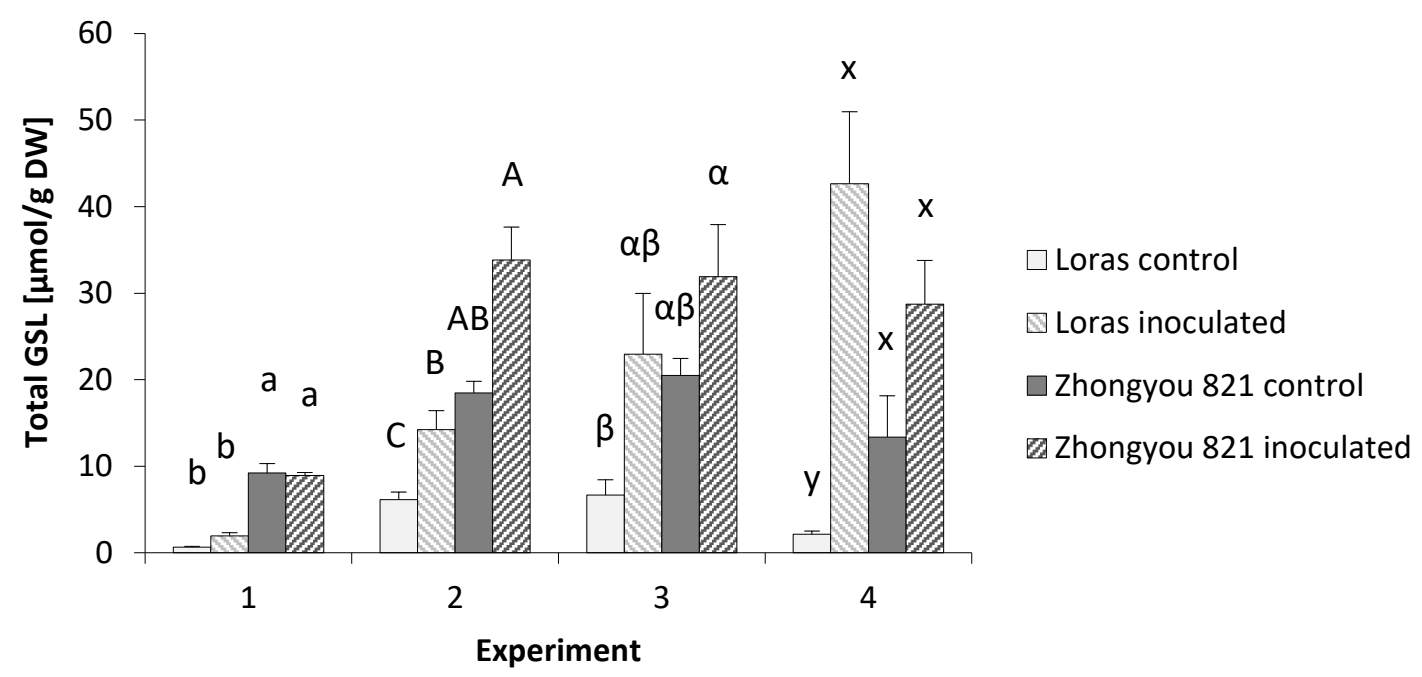

Fig. 43 Total glucosinolate content in the stem of two oilseed rape genotypes Loras and Zhongyou 821 in control plants and after inoculation with S. sclerotiorum in four independent experiments. Means and standard errors are shown. Letters indicate significant differences (Kruskal-Wallis ANOVA, $P<0.05$ ).

The graph above already shows an influence of genotypes and inoculation, but regardless of the plant tissue. Moreover, in control plants the genotypic difference between Loras and Zhongyou 821 stands out. Univariate test of significances for main effects on the total GSL content of stem and root of two oilseed rape genotypes after inoculation with S. sclerotiorum showed no influence of the day post inoculation. Therefore, effects of genotype, inoculation and plant tissue on total glucosinolates were tested (Tab. 18). Both effects of genotype and plant tissue were highly significant. Data for GSL content in the hypocotyl can be found in the annex (Fig. 1A, Fig. 2A and Tab. 12A). The inoculation had a significant effect, too. Since the root was of higher interest than the hypocotyl, the results of stem and root inoculation were selected. In the following, all effects on total GSL comparing stem and root are described in detail. 
Tab. 18 F-values of main effect ANOVA of genotype, inoculation, plant tissue and the interaction on the total glucosinolate content including the degrees of freedom (df). Asterisks represent differences according to significance level (ANOVA: $n=4 ;{ }^{* *} p<0.001,{ }^{* *} p<0.01,{ }^{*} p<0.05$ and ns not significant).

\begin{tabular}{lll}
\hline Effect & df & Total GSL \\
\hline Genotype & 1 & $50.75^{* * *}$ \\
Inoculation & 1 & $5.65^{*}$ \\
Plant tissue & 1 & $118.55^{* * *}$ \\
Genotype $\times$ Inoculation & 1 & $5.63^{*}$ \\
Genotype $\times$ Plant tissue & 1 & $1.62^{\text {ns }}$ \\
Inoculation $\times$ Plant tissue & 1 & $2.47^{\text {ns }}$ \\
Genotype $\times$ Inoculation $\times$ Plant tissue & 1 & $0.15^{\text {ns }}$ \\
\hline
\end{tabular}

The described effects were also visible comparing the total GSL content as means over dpi corresponding to the detailed effects (Fig. 44). The significant genotypic effect appeared in Zhongyou 821 containing more GSL than Loras, especially in the root. This leads to the plant tissue effect with the root generally having higher amounts than the stem, being significant in both genotypes. The inoculation had no consistent effect but the tendency of a slight increase of total GSL can be seen.

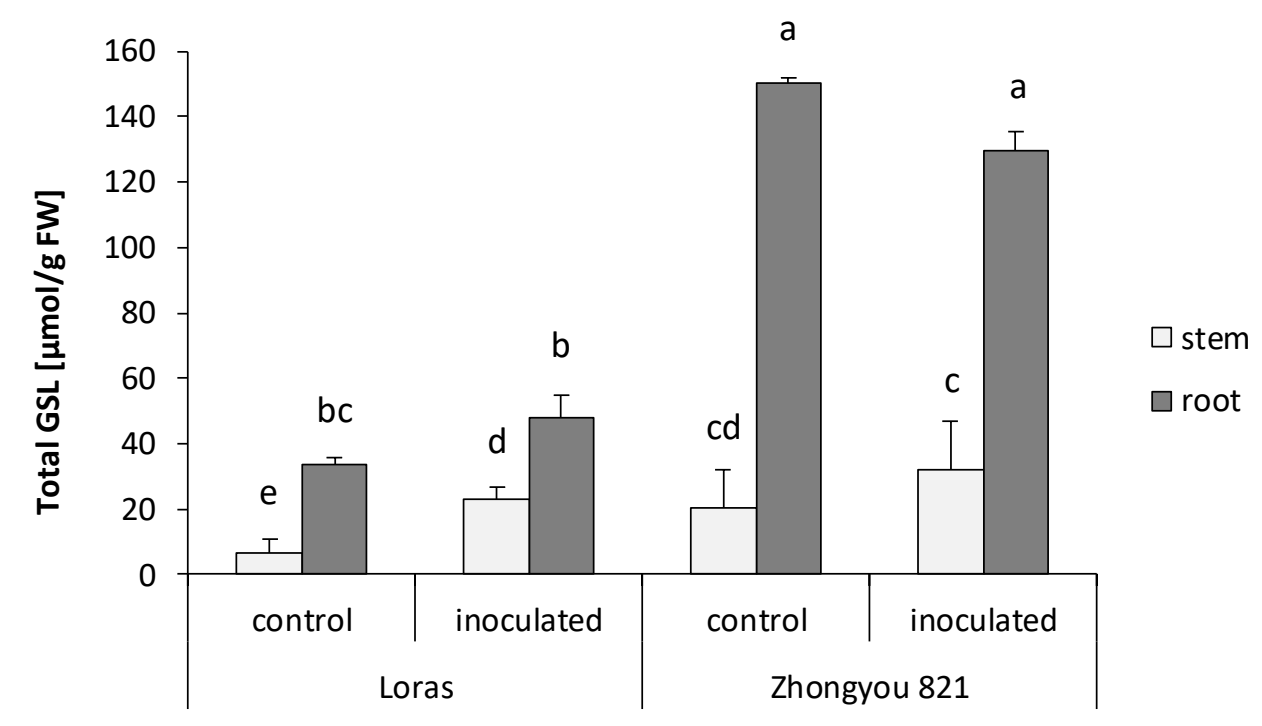

Fig. 44 Total GSL content in stem and root of the oilseed rape genotypes Loras and Zhongyou 821 after inoculation with S. sclerotiorum compared to control plants. Means values and standard errors are shown. Letters indicate significant differences (LSD test: $\mathrm{n}=3, P<0.05$ ). 
Total GSL represent thirteen glucosinolates being identified and quantified in healthy and S. sclerotiorum inoculated plants within different tissues of the oilseed rape genotypes Loras and Zhongyou 821. GSL were identical over all experiments and are listed in Tab. 19.

Tab. 19 Abbreviations, chemical names, common names and groups of glucosinolates identified in stem, root and hypocotyl of oilseed rape plants.

\begin{tabular}{|c|c|c|c|}
\hline Group & Abbreviation & Chemical name & Common name \\
\hline \multirow{9}{*}{$\begin{array}{l}\stackrel{U}{0} \\
\frac{0}{0} \\
\frac{0}{0} \\
\frac{.0}{\bar{\alpha}}\end{array}$} & $3 m t p$ & 3-methylthiopropyl & Glucoiberverin \\
\hline & $4 \mathrm{mtb}$ & 4-methylthiobutyl & Glucoerucin \\
\hline & $5 m t p$ & 5-Methylthiopentyl & Glucoberteroin \\
\hline & $3 m s p$ & 3-methylsulphinylpropyl & Glucoiberin \\
\hline & $4 m s b$ & 4-methylsulphinylbutyl & Glucoraphanin \\
\hline & $5 m s p$ & 5-methylsulphinylpentyl & Glucoalyssin \\
\hline & 3but & 3-butenyl & Gluconapin \\
\hline & 4 pent & 4-pentenyl & Glucobrassicanapin \\
\hline & $40 h b$ & 4-hydroxybutyl & Glucoarabidopsisthalianain \\
\hline \multirow[t]{2}{*}{ Aromatic } & $2 \mathrm{PE}$ & 2-phenylethyl & Gluconasturtiin \\
\hline & I3M & Indol-3-ylmethyl & Glucobrassicin \\
\hline \multirow{2}{*}{$\begin{array}{l}\frac{0}{0} \\
\frac{0}{0}\end{array}$} & 4MOI3M & 4-methoxyindol-3-ylmethyl & 4-Methoxyglucobrassicin \\
\hline & NMOI3M & $N$-methoxyindol-3-ylmethyl & Neoglucobrassicin \\
\hline
\end{tabular}

GSL were identified and quantified in the roots. According to the effect of the experimental factors genotype, treatment and/or plant part, the individual GSL could be grouped (Fig. 45, see also annex Tab. 13A). The first group assembles the compounds being present in higher concentrations in the root than in the stem. Compared to the stem, the root revealed higher concentrations of glucoiberverin, glucoerucin, glucoberteroin and glucoraphanin. This finding was confirmed in the repetition of experiment (see annex Fig. $3 \mathrm{~A}$ and Fig. 4A). Comparing the tissues in regard of glucoiberin and gluconapin, higher amounts were found in the stem than in the root, which was true for both genotypes and independent from the inoculation. Also, glucoalyssin and glucobrassicanapin were higher 
in the stem except at $7 \mathrm{dpi}$. The result was contrary for glucoarabidopsisthalianain, where first amounts were higher in the root and increased in the stem at $7 \mathrm{dpi}$. Differences were not significant. In the repeated experiment, glucoiberin and gluconapin but also glucobrassicanapin showed higher concentrations in the stem (see annex Fig. 3A and Tab. 14A). However, there was no distinct effect on glucoraphanin, glucoalyssin and glucoarabidopsisthalianain.

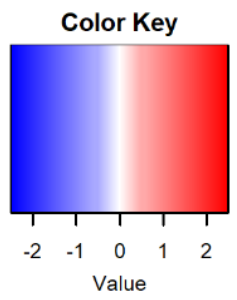

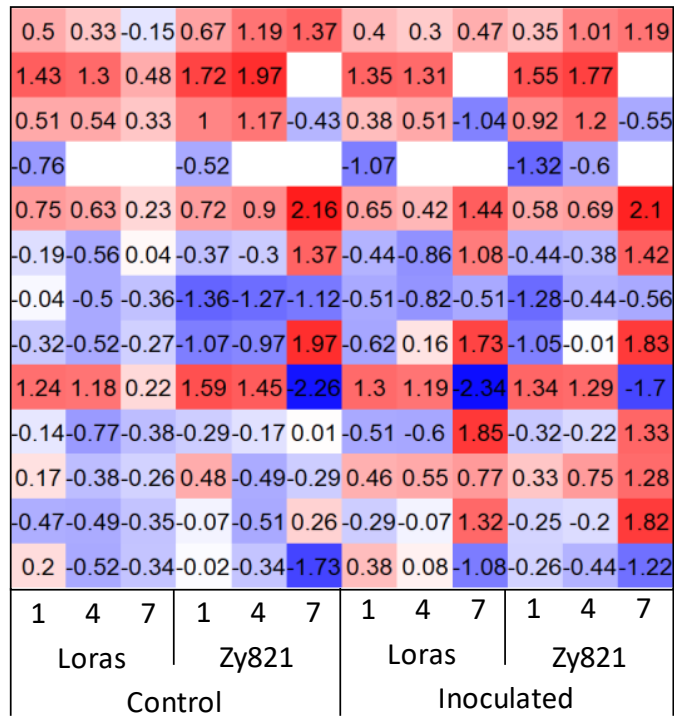

Glucoiberverin Glucoerucin Glucoberteroin Glucoiberin Glucoraphanin Glucoalyssin Gluconapin Glucobrassicanapin Glucoarabidopsisthalianain Gluconasturtiin Glucobrassicin 4-methoxyglucobrassicin Neoglucobrassicin

Fig. 45 Relation of glucosinolates in roots compared to stems of oilseed rape genotypes Loras and Zhongyou 821 (Zy821) non-inoculated and inoculated at 1, 4 and $7 \mathrm{dpi}$ with S. sclerotiorum.

Comparing GSL amounts of infected to non-infected plants there was an effect at $7 \mathrm{dpi}$ in stem and root of Loras and in the root of Zhongyou 821 (Fig. 46). Indole GSL increased rapidly after inoculation and were affected independently by the genotype or the plant tissue. This result was confirmed in the repeated experiment (see annex Fig. 4A and Tab.14A). No significant differences were observed in both the first and second repetition. 


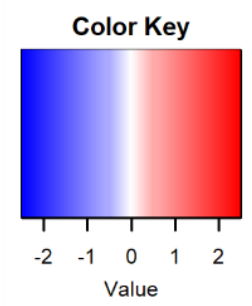

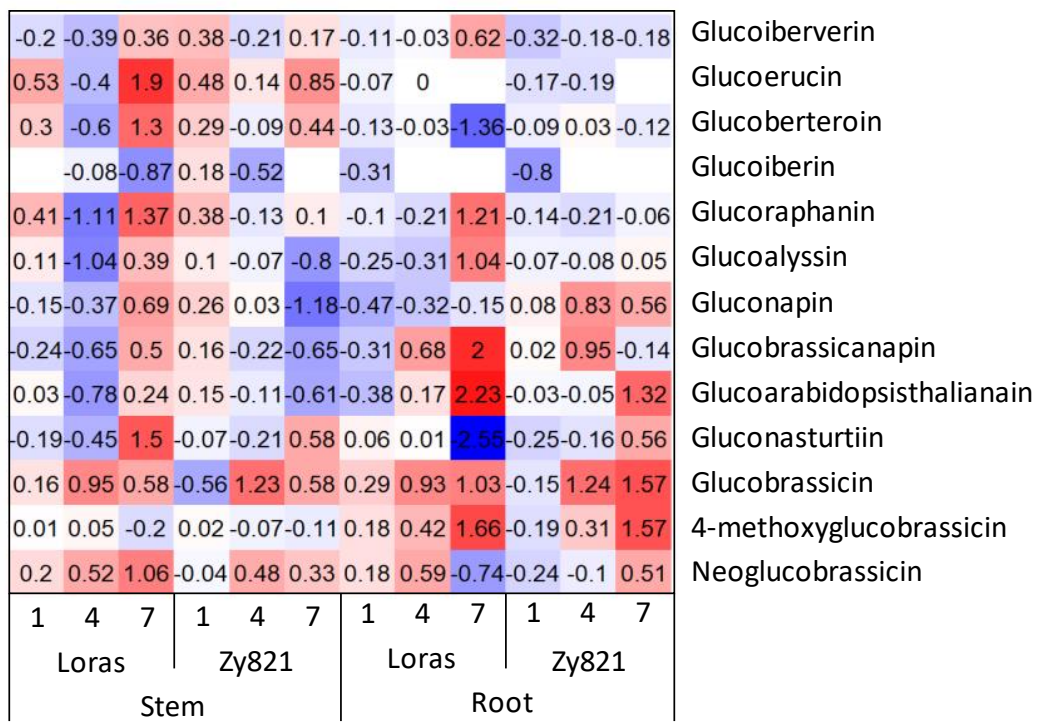

Fig. 46 Effect of stem and root inoculation with S. sclerotiorum on glucosinolates of the oilseed rape genotypes Loras and Zhongyou 821 (Zy821) at 1, 4 and 7 dpi.

\section{Glucosinolate pattern in infected and non-infected plants of different genotypes}

Glucosinolates were analysed in different genotypes from the Brassica napus Diversity Fixed Foundation Set. For this purpose, the disease assessment was conducted again, but the results for lesion length did not correspond to the first screening (Tab. 20, compare 3.1 Screening Brassica germplasm for genotypic resistance ). 
Tab. 20 Length of lesion 7 days post inoculation with S. sclerotiorum of different genotypes generated in two independent screenings. Mean values and standard errors are shown $(n=20)$.

\begin{tabular}{llll}
\hline \multirow{2}{*}{ Number } & \multirow{2}{*}{ Name } & \multicolumn{2}{c}{ Lesion length [mm] } \\
& & Screening 1 & Screening 2 \\
\hline BnASSYST-231 & SWU Chinese 3 & $36.7 \pm 25.2$ & $3.8 \pm 14.3$ \\
& Zhongshuan 9 & - & $36.4 \pm 14.7$ \\
BnASSYST-251 & Karat & $90.4 \pm 25.6$ & $52.7 \pm 36.8$ \\
& Zhongyou 821 & $34.1 \pm 25.8$ & $68.3 \pm 16.3$ \\
BnASSYST-326 & Furax & $10.2 \pm 9.1$ & $76.8 \pm 14.9$ \\
BnASSYST-300 & Magma & $105.1 \pm 33.4$ & $83.1 \pm 15.1$ \\
BnASSYST-240 & Westar (DH) & $58.2 \pm 35.8$ & $87.8 \pm 17.0$ \\
BnASSYST-275 & Duplo & $88.8 \pm 34.4$ & $89.3 \pm 29.1$ \\
& Loras & $76.2 \pm 24.1$ & $99.5 \pm 1.1$ \\
\hline
\end{tabular}

In the second screening, only Magma, Westar, Duplo and Loras were comparable to the first screening data showing long lesion length after fungal infection. The other genotypes showed either lower (SWU Chinese 3, Karat) or higher (Zhongyou 821) values in the second screening compared to the first. According to their susceptibility occurring in the second screening, the genotypes are ranked in the following graph showing GSL content in inoculated stem and non-inoculated stem and root (Fig. 47). In all genotypes except SWU Chinese 3, the inoculation was accompanied by an increase of total GSL. Roots in noninoculated plants $(\mathrm{Ctl})$ generally had significantly higher total GSL amounts than the stem. No correlation was found between the lesion length and amount of total GSL. 


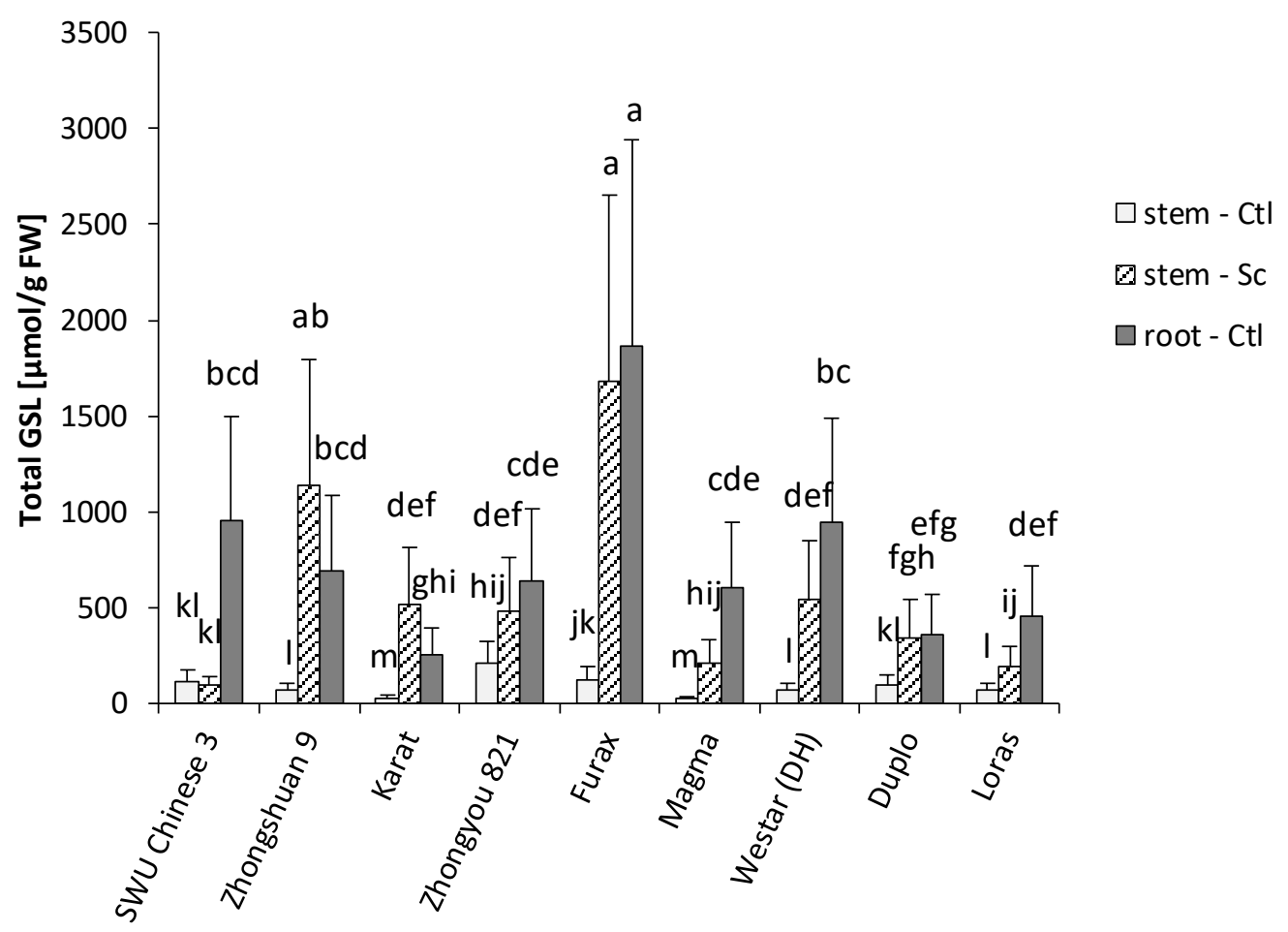

Fig. 47 Total glucosinolates in non-inoculated (CtI) roots and stems and inoculated (Sc) stem of different oilseed rape genotypes at 7 days post inoculation with $S$. sclerotiorum. Mean values and standard errors are shown. Letters indicate significant differences (LSD test: $\mathrm{n}=3, P<0.05$ )

Genotype, inoculation and plant tissue had significant effects on the content of total GSL (Tab. 21).

Tab. $21 \mathrm{~F}$-values of main effect ANOVA of the factors genotype, inoculation and plant tissue on the total GSL content including the degrees of freedom (df). Asterisks represent differences according to significance level (ANOVA: $n=3 ;{ }^{* *} p<0.001,{ }^{* *} p<0.01,{ }^{*} p<0.05$ and ns not significant).

\begin{tabular}{lcl}
\hline Effect & df & Total GSL \\
\hline Genotype & 8 & $7.97^{* * *}$ \\
Inoculation & 1 & $121.34^{* * *}$ \\
Plant tissue & 1 & $187.60^{* * *}$ \\
\hline
\end{tabular}




\subsection{Effect of growth regulators on development of plants and disease}

The effect of two growth regulators and two fungicides with growth regulatory efficacy on oilseed rape plant morphology and level of sclerotinia stem rot disease was investigated. No effect on the developmental stage but a reduction of shoot elongation was observed 20 days after application (Fig. 48 and Fig. 49). The stem diameter was affected, and the stem lesion length caused by S. sclerotiorum was reduced (Fig. 50 and Fig. 51).

The application of growth regulators had no effect on plant developmental stage. There was a small genotypic effect as Zhongyou 821 generally showed a slower development compared to Loras (Fig. 48). In the water control, Zhongyou 821 reached BBCH of 66 to 67 meaning that the plants were in full flower and petals were already falling down. Loras plants already were in stage 69 towards the end of bloom. The treatment, however, did not result in significant differences among development stages of the two genotypes. When the experiment was repeated, the effect of growth regulators on $\mathrm{BBCH}$ was less strong but showed the same tendency (see annex Tab. 15A).

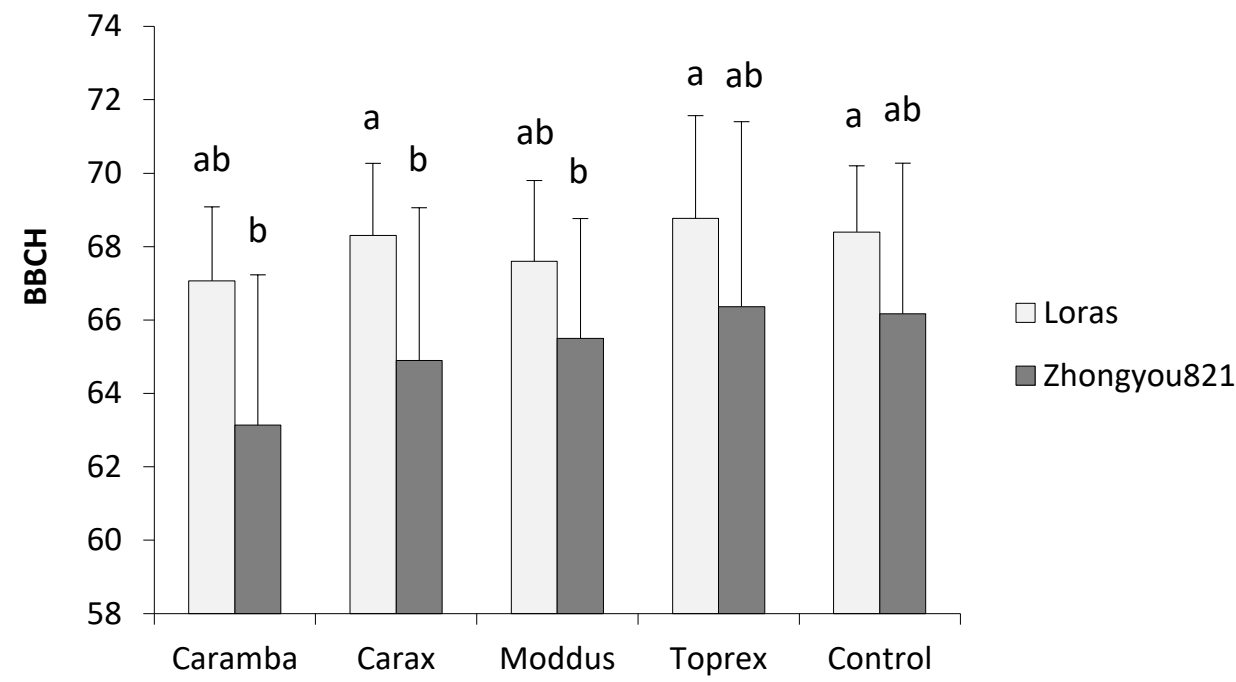

Fig. 48 Effect of different growth regulators on growth stage $(B B C H)$ of oilseed rape plants 20 days after application on the genotypes Loras and Zhongyou 821. Bars show mean values. Error bars represent standard deviation. Letters indicate significant differences (Kruskal Wallis ANOVA: $n=30$, $P<0.05)$.

The plant height was significantly reduced following growth regulator application in both genotypes (Fig. 49). The effect showed a moderate correlation $\left(R_{s}=0.32\right)$. However, the reduction effects caused by different growth regulators were identical. In the control, Loras and Zhongyou 821 reached an average height of $915 \mathrm{~mm}$ and $720.3 \mathrm{~mm}$, respectively. After 
application of Caramba, the plant height of Loras was reduced by $20.8 \%$ to $724.3 \mathrm{~mm}$, and of Zhongyou 821 by $35.2 \%$ to $467.0 \mathrm{~mm}$. This reduction is representative for all treatments. Results for plant height were confirmed in the repetition of the experiment (see annex Tab. $15 A)$.

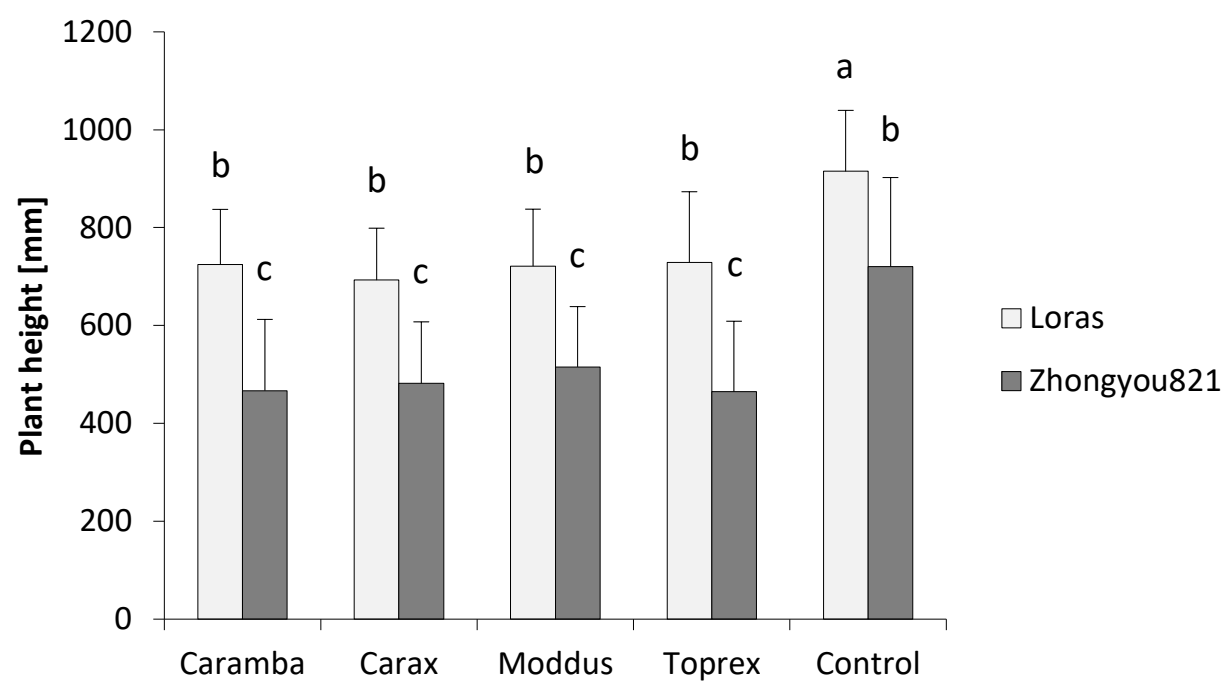

Fig. 49 Effect of different growth regulators on plant height of oilseed rape plants at flowering stage, 20 days after application on the genotypes Loras and Zhongyou 821. Bars show mean values. Error bars represent standard deviation. Letters indicate significant differences (LSD test: $\mathrm{n}=30, P<0.05$ ).

The stem diameter of both genotypes was affected by application of the growth regulator Moddus (Fig. 50). After application of Moddus, the stem diameter was reduced by more than $17 \%$. Toprex had a significantly reducing effect on the stem diameter of Loras. However, other growth regulators did not alter the stem diameter compared to the control. The repetition of experiment did not show any effect of growth regulator application on the stem diameter (see annex Tab. 15A). 


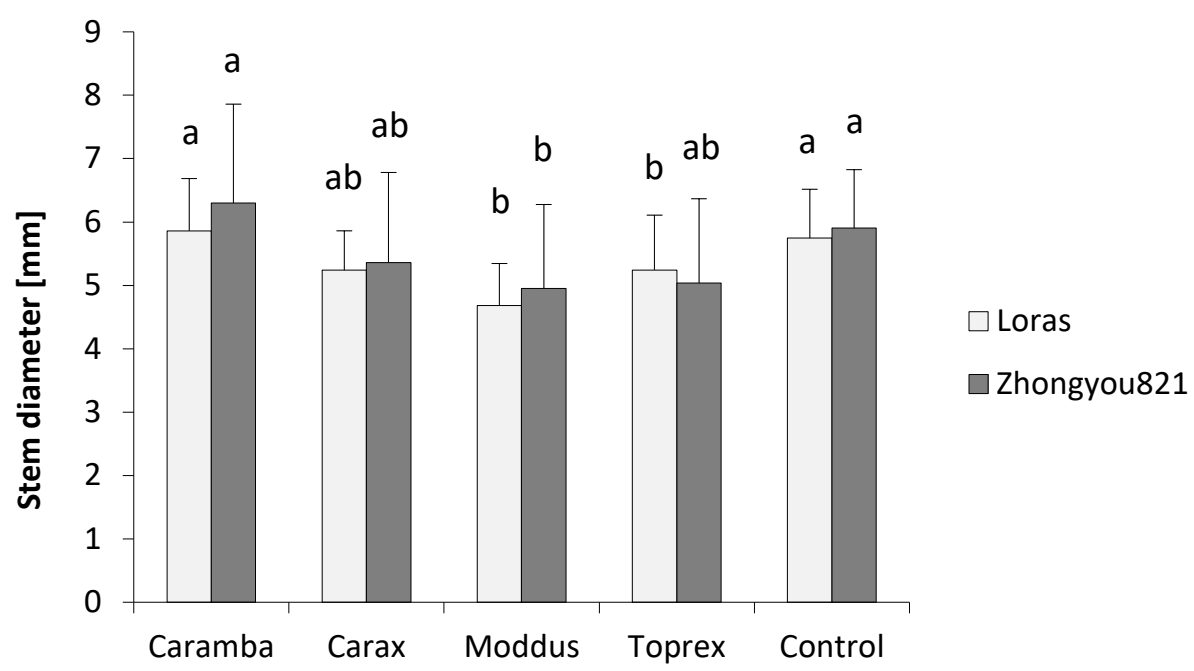

Fig. 50 Effect of different growth regulators on stem diameter of oilseed rape plants 20 days after application on the genotypes Loras (susceptible) and Zhongyou 821 (partially resistant). Bars show mean values. Error bars represent standard deviation. Letters indicate significant differences (Kruskal-Wallis ANOVA: $\mathrm{n}=30, P<0.05$ ).

Generally, lesions on Zhongyou 821 were significantly smaller than on Loras. The previous application of the growth regulators Caramba and Moddus at the beginning of stem elongation showed an effect on the length of lesions developing after inoculation with S. sclerotiorum of the stem of Zhongyou 821 during flowering stage (Fig. 51). After application of Moddus, lesions on Zhongyou 821 were only half as long as on control plants, while Caramba even caused a greater reduction of the lesion length by two thirds, compared to the control. The application of growth regulators did not influence the length of stem lesions in the susceptible genotype Loras. 


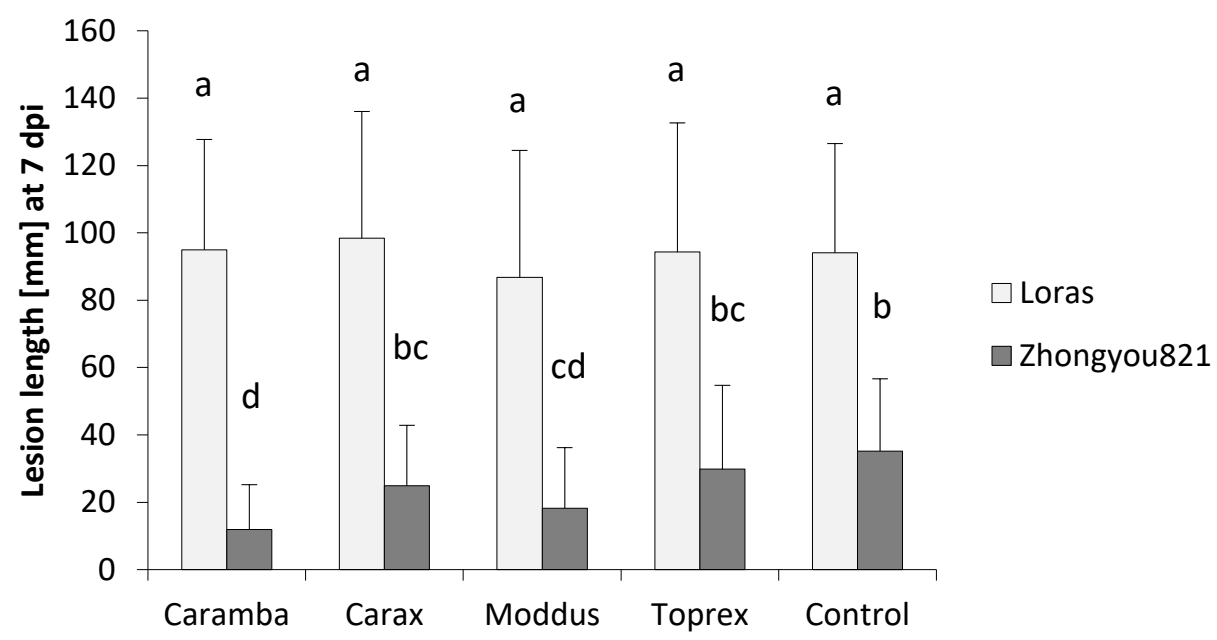

Fig. 51 Effect of different growth regulators on lesion length of S. sclerotiorum on oilseed rape at $7 \mathrm{dpi}, 20$ days after application on the genotypes Loras (susceptible) and Zhongyou 821 (partially resistant). Error bars represent standard deviation. Letters indicate significant differences (KruskalWallis ANOVA: $\mathrm{n}=30, P<0.05)$.

The repetition of experiment also showed that growth regulators can affect lesion development. Values tended to be comparable, but results could not be proven as significant which was attributable to high standard deviations (see annex Tab. 15A).

The variation in plant development could be partly explained by the application of different growth regulators. Precisely, reduction in height was explained by $32 \%$, and $25 \%$ of the effect were explained in case of $\mathrm{BBCH}$. In addition, plant height and growth stage were strongly correlated with the lesion length with $R_{s}=0.60(P<0.01)$ and $R_{s}=0.50(P<0.01)$, respectively. 


\section{Discussion}

Sclerotinia sclerotiorum is a devastating pathogen and the causal agent of sclerotinia stem rot. Favourable conditions for the pathogen can lead to high yield losses (Adams \& Ayers, 1979; Boland \& Hall, 1994). Pest management is performed via direct disease control with fungicides potentially having an adverse effect on the environment (Taylor et al., 2015; Derbyshire \& Denton-Giles, 2016). So far, no resistant oilseed rape (OSR) cultivars have been bred. Therefore, possibilities to improve resistance of OSR cultivars against sclerotinia stem rot were explored in this study. In a first approach, resistance sources were sought in the gene pool by screening and disease assessment on a set of Brassica napus accessions under controlled conditions. Since comparison of genotypes turned out to show only weak and inconsistent responses to infection, an alternative approach was chosen. In the following, two genotypes performing contrasting levels of susceptibility were investigated for their interaction with the pathogen in more detail. In this regard, tissue specific disease response was investigated. The plant stem as the preferred target tissue of infection was compared to the hypocotyl and the root in order to find out whether resistance against S. sclerotiorum is relying on tissue specificity, answering the question which basic physiological and histological traits lead to higher resistance of a specific plant tissue. Possible defence mechanisms such as accumulation of phenolic compounds, change in peroxidase activity and alteration in glucosinolate pattern due to inoculation were studied. Moreover, colonisation pattern of fungal hyphae in the different plant tissues was observed. Last but not least, the tissue specific morphological effect was simulated by application of growth regulators.

\subsection{Lack of complete genotypic resistance against S. sclerotiorum}

Brassica napus is a relatively young species originated from only few interspecific hybridisations between B. rapa L. (turnip rape) and B. oleracea L. (cabbage). This led to only low genetic diversity (Kimber \& McGregor, 1995; Iñiguez-Luy \& Federico, 2011). The gene pool was further narrowed down when breeding went through a bottle neck in terms of enhanced seed quality (Becker et al., 1995; Bus et al., 2011). This resulted into a reduced variation in B. napus and makes breeding for resistant lines even more difficult. Indeed, the 
resistance level of OSR against $S$. sclerotiorum is reported to range between those of its parental species, namely B. oleracea with a high and B. rapa with a low resistance level (Mei et al., 2011). Since in B. napus no promising resistance sources were found, researchers focussed on exotic Brassica species like $B$. juncea, $B$. rupestris, $B$. insularis, $B$. montana, B. villosa, B. carinata and B. nigra showing higher levels of resistance (Navabi et al., 2010; Mei et al., 2011; Uloth et al., 2016). As the C-genome set harbours higher resistance, resynthesised lines with $B$. oleracea as C-genome donor exhibit a good stem resistance (Ding et al., 2013). This can be explained by the findings of Mei et al. (2013). They mapped quantitative trait loci (QTL) for Sclerotinia resistance on the C09 chromosome of B. incana, a wild form of $B$. oleracea. However, no equivalents could be found on N19, the chromosome in OSR corresponding to C09 (Zhao \& Meng, 2003b; Zhao et al., 2006; Yin et al., 2010). This finding indicates that resistance was not transferred into the $B$. napus background. Recently, efforts were put on introgression lines to introduce resistance genes from other plant families resulting in a higher potential of resistance (Garg et al., 2010a; Barbetti et al., 2011; Ding et al., 2013). This material yet needs to be adapted in elite breeding programmes to achieve suitable oilseed rape cultivars with double-low quality.

For the present study only B. napus accessions were used in order to screening for resistance against $S$. sclerotiorum. Identifying promising $B$. napus candidates may facilitate complex breeding strategies. Furthermore, this study enlarges today's knowledge about resistance sources in OSR. The accessions were chosen from the Brassica napus Diversity Fixed Foundation Set (BnaDFFS). For BnaDFFS, out of thousands of genetic resource accessions, a representative subset was selected (see www.brassica.info). This subset served as a basis to generate founder lines. Out of them, fixed lines were developed by DH or single seed descent. The set provides a vast diversity of genotypes, representing different growth types (winter and spring OSR, kale, swede and Chinese varieties) to allow finding resistance sources within a broad gene pool.

The different growth types displayed no specific pattern of response to the inoculation. Independent from growth types, all genotypes performed both, smaller and bigger lesions. Hence, none of the growth types was particularly less or more susceptible compared to the others. Most genotypes (101 out of 183) were susceptible, displaying intermediate to long lesion lengths and high disease scores. Such disease scores of eight or higher describe a 
lesion girdling the plant's stem, leading to death. Several genotypes showed comparably smaller lesion lengths and lower disease scores, seemingly owing a higher capability to resist infection by S. sclerotiorum. However, it must be clarified that inoculation was successful on all genotypes and the subsequent symptom development is attributed to susceptibility. Consequently, no candidates being resistant against S. sclerotiorum were found, confirming other studies reporting a lack of genotypic resistance sources (Liu, 2004; Bradley et al., 2006a; Derbyshire \& Denton-Giles, 2016).

Most genotypes flowered nearly simultaneously. The first reached the flowering stage three weeks after vernalisation, i.e. 13 weeks after sowing. For spring types, the time to flowering lasted ten weeks. Within the following eight weeks, genotypes reached the flowering stage successively, thus being inoculated in constant time intervals. However, some genotypes displayed an 'atypical' phenotype. When the majority of genotypes had already flowered, the atypical types still remained in the vegetative stage. Even after an extended time period under conditions favouring generative growth they did not reach flowering stage. Most of the atypical genotypes finally produced an elongated but tough and hard stem having lost the leaves due to senescence except for the younger leaves near the growth zone at the tip of the stem.

Genotypes revealing atypical phenotypes showed less severe responses to infection. The short, tough stems were inappropriate for inoculation and made screening difficult. Since plant cells were more lignified than in the younger plants in full blossom, the cell walls provided a stronger barrier for the fungus. At the beginning of stem elongation, cell division takes place in the meristem zone and is followed by cell elongation (Sachs et al., 1959; Beemster \& Baskin, 1998). The atypical plants were less than half as high as the 'normal' genotypes. Therefore, it can be assumed that the plant cells also were shorter. These shorter cells created a compact tissue less easy to be overcome by hyphae meeting cell wall barriers more frequently. As a result, values for lesion lengths and disease scores were lower than in the 'normal' genotypes. Moreover, there is evidence that flowering stage is particularly vulnerable to infection with S. sclerotiorum. Negative correlations were found between flowering time and resistance, supported by the fact that QTL for both traits are colocalising (Zhao et al., 2006; Mei et al., 2013). This might explain why 'normal', flowering plants showed notably high susceptibility. To achieve a better quality of data and ensure 
homogeneity of the outcome, inoculation and disease assessment of all genotypes within 6 to 8 weeks after vernalisation might be conducive. Apart from these experimental constraints, the disease assessment confirms reports of other researchers about the lack of genotypic resistance in cultivated compared to wild Brassica species (Mei et al., 2011; Ding et al., 2013; Uloth et al., 2013; You et al., 2016, Taylor et al., 2018).

Loras and Zhongyou 821 were included as reference genotypes in all four screening runs to allow comparison of the results. However, the references were not responding consistently to infection. Zhongyou 821, proven in former experiments and reported in the literature as a partially resistant line (Li et al., 1999c; Wang et al., 2004; Zhao et al., 2009; Höch, 2016) surprisingly suffered from higher lesion lengths and disease scores as expected. Loras also showed high variability between the runs. The unpredictability of disease response of the reference lines leads to uncertainty concerning other accessions. Since a reference is lacking it is difficult to judge whether accurate or unusual disease symptoms developed in the tested genotypes. Alteration in the disease response of reference genotypes suggests heterogeneous responses of all genotypes and is contradictory to the need of experimental reproducibility. Therefore, reproducibility of disease assessment on flowering oilseed rape infected with S. sclerotiorum via stem inoculation is not assured. Inconsistency in disease assessment results for resistance against S. sclerotiorum has been reported before (Pratt et al., 1988; Kull et al., 2003; Li et al., 2007) and can be explained by its quantitative nature. In view of this fact, the screening method has to be discussed. Screening methods are not yet standardised and hence difficult to compare (Derbyshire \& Denton-Giles, 2016). Thus, research still involves finding the right procedure. In nature, the plant is infected by ascospores landing on senescent petals, which then fall on leaf axils (Abawi \& Grogan, 1979; Lumsden, 1979; Derbyshire \& Denton-Giles, 2016). From there the pathogen spreads directly or via the petiole into the stem. In this study, inoculation was carried out via a mycelium overgrown agar plug attached on an injured area on the stem. The injury was needed to ensure homogeneous responses among accessions. Unlike most studies this did not necessitate an injury before placing the mycelium overgrown agar plug (Li et al., 2004, 2007; Ge et al., 2012; Uloth et al., 2013). Puncturing the stem surface already removes the first barrier that resists fungal invasion (Yeats \& Rose, 2013; Seifbarghi et al., 2017). It is thus possible that the applied method bypasses certain resistance mechanisms triggered 
earlier, e.g. when penetration of the epidermis happens (Uloth et al., 2014; Derbyshire \& Denton-Giles, 2016). Furthermore, it can be questioned if mycelium on half concentrated potato dextrose agar (PDA) is the right method of choice. In other studies, macerated mycelium in liquid media is described as inoculum, allowing a directed penetration and determinisation of an exact concentration of inoculum (Garg et al., 2008). Moreover, compared to petals as nutrient source, PDA might be a too complex medium. Nevertheless, many studies rely on stem inoculation for disease assessment on intact flowering plants using mycelium on PDA.

Studies on resistance of $B$. napus against $S$. sclerotiorum have been performed since the 1980 s and have shown its complex nature. They indicate the assumption that resistance can be either monogenic or polygenic (Abawi et al., 1978; Zhao \& Meng, 2003a; Zhao et al., 2006). In addition, segregation patterns of generations following inter-specific crosses have suggested that resistance is of quantitative nature (Disi et al., 2014). Moreover, results of many studies designate that resistance is under strong environmental influence (Zhao \& Meng, 2003a; Zhao et al., 2006; Yin et al., 2010), thus corroborating its quantitative character. To elucidate defence responses in detail, genomic approaches have been performed. There are various genes encoding for enzymes contributing to QDR (French et al., 2016). They are located on quantitative trait loci (QTL). Such QTL for S. sclerotiorum resistance have been found in several independent studies (Zhao \& Meng, 2003a; Zhao et al., 2006; Mei et al., 2013; Wu et al., 2013; Wei et al., 2014; Li et al., 2015). QTL for stem resistance were detected on different chromosomes, among which two conserved QTL regions on $A 9$ and $C 6$ for stem resistance were found (Li et al., 2015). The problem of identifying markers for QTL remains in the screening method. Studies include inoculation on different plant tissues at different growth stages yielding inconsistent results (Zhao \& Meng 2003a; Zhao et al., 2006; Ding et al., 2013; Uloth et al., 2013; You et al., 2016). This corresponds with other studies where no correlation between stem and leaf resistance was found (Zhao \& Meng, 2003a; Taylor et al., 2018). It is hence assumed that resistance in different tissues may be based on differential sets of genes, showing again the complexity of resistance in $B$. napus against $S$. sclerotiorum.

In conclusion, among a set of 183 accessions of B. napus screened in the present study, no sources for genotypic resistance against S. sclerotiorum were found. The small variability 
within the $B$. napus gene pool may explain the little variation between the genotypes screened and why most displayed intermediate or strong susceptibility. Identification of resistance sources and QTL for disease resistance is a complex task and needs further investigation. The quest for more efficient disease assessment studies in terms of both time and feasibility leads to the next point where alternative methods are discussed.

\subsection{Alternative methods for disease assessment}

Screening was performed after inoculation of the stem of flowering plants, requiring vernalisation for winter OSR generally taking a long time. This method has been chosen because it is close to natural conditions. Taylor et al. $(2015,2018)$ describe leaf ascospore inoculation as a suitable means to determine stem resistance. Hence, to find a possibility to shorten the screening procedure in this study, leaf screening was tested. Again, mimicking infected petals was carried out by placing mycelium overgrown agar plugs on the first and second leaves of three weeks old plants. The accessions chosen for the leaf screening gave contrasting results in the initial stem screening. However, accessions being less susceptible in the stem screening did not display the same response to inoculation in the leaf screening.

It has been shown that plant tissues such as cotyledons are appropriate to show accurate responses to inoculation corresponding to field results (Garg et al., 2008, 2010b). However, several authors describe a lack of correlation between leaf and stem infection (see 4.1 Lack of complete genotypic resistance against S. sclerotiorum). Moreover, the first and second leaves as used in this study seem to be inappropriate because they do not sufficiently resemble later emerging leaves. Other tests were performed on the fourth leaves of plants with at least eight leaves (Mei et al., 2011; Taylor et al., 2018). Probably, using at least the third and fourth leaves would have shown disease responses with higher accordance to the stem screening. Some accessions having very hairy leaves impeded a good attachment of the agar plugs on the leaf surface. This led to high standard errors. Thus, mycelium fragments or ascospores in liquid media might be more adequate than agar plugs for leaf inoculation.

Another possible inoculation method for disease assessment is the petiole technique (Zhao et al., 2004; Taylor et al., 2015). For this method, petioles of the third fully expanded leaf 
are severed $2.5 \mathrm{~cm}$ from the main stem using a razor blade. Then, the petiole is forced through a PDA disc overgrown with S. sclerotiorum mycelium placed in a 1,000- $\mu$ l pipette tip. The infection spreads along the petiole into the stem, where symptoms can be assessed. Plants at early leaf stage can be used, accommodating a short screening period. Hence, the petiole represents a compromise between leaf and stem inoculation. Altogether, detailed comparative studies of leaf, petiole and stem inoculation for disease assessment studies should be performed to either confirm or disprove correlation between susceptibility of different plant tissue.

Results about the comparability between different inoculated plant tissues have been contradictory in the present and former studies (Garg et al., 2008, 2010b; Zhao \& Meng 2003a; Uloth et al., 2013; Taylor et al., 2018). Disease assessment on stems of flowering OSR plants remain the best choice as it is the closest to natural conditions. This developmental stage represents a specific situation and involves genes, hormones and chemical compounds which are held ready in case of pathogen attack. In addition, the plants stay intact allowing analyses on biochemical compounds unaltered due to wounding. Therefore, future experiments should concentrate on the stem inoculation method.

\subsection{Disease development in different plant tissues}

No resources for resistance against Sclerotinia stem rot disease in 183 different accessions belonging to the Brassica napus Diversity Fixed Foundation Set were identified. This was caused by the lack of genotypes with contrasting susceptibility to the disease. As a consequence, more attention was directed to a comparison of different plant tissues. The tissue of stem, root and hypocotyl was investigated to achieve a sufficiently pronounced diversity in responses to stem rot infection. Finding different resistance levels based on tissue specificity might counterbalance the lack of genotypic variability.

In the field, OSR is usually infected at the stem via ascospores (Abawi \& Grogan, 1979; Lumsden, 1979; Bolton et al., 2006). Unlike other plant species like sunflower, in OSR myceliogenic germination of sclerotia followed by direct infection of the root has been only seldomly reported (Wulf, 2011). Thus, the assumption was made that the root is resistant to the infection by S. sclerotiorum. To gain certainty about variability in tissue specific disease response, studies on the symptom development and disease quantification in 
different plant tissues were performed. Since to the author's knowledge this is the first time that investigations on tissue specificity of resistance in OSR against S. sclerotiorum are made, not only the results themselves are discussed but also the methodological background.

\subsubsection{Symptom development}

For disease assessment on the different plant tissues stem, hypocotyl and root of OSR plants were inoculated. The inoculation method via a mycelium overgrown agar plug was the same for all tissues. Two genotypes were chosen based on former experiences about the intensity of disease symptom development: Loras, a highly susceptible spring type, and Zhongyou 821, a partially resistant Chinese intermediate type (Wang et al., 2004). It can be generally stated that responses to the infection by S. sclerotiorum vary strongly between independent experiments. With respect on genotypic effects, Zhongyou 821 was less susceptible than Loras in the first experiment. However, this already weak contrast disappeared from the second experiment on. Since during the experimental procedure nothing was changed, this cannot be explained but leads to the loss of the experimental factor 'genotype' for the following experiments.

In the first experiments, the tap root, the characteristic root formed by OSR, did not develop properly. Plants showed a root ball unsuitable for inoculation. For this reason, the hypocotyl served as the plant tissue to be compared to the stem. Concerning the plant tissue effect, in the first experiment the stem was less susceptible than the hypocotyl. The contrary was found in the repetition, but both results were not significant. Also, the overall lesion length differed strongly between the two experiments. All experiments were conducted in the greenhouse to provide a controlled environment and stable conditions. Nonetheless, seasonal climate seems to influence at least one of the organisms - host plant or pathogen. This might be the reason for interexperimental differences in terms of symptom development. Thus, to answer the question whether the stem or the hypocotyl is more susceptible to infection with S. sclerotiorum, more experiments are needed. These should be accomplished during the same period of the year to avoid environmental influence on the greenhouse conditions. Also, they should include the differentiation between wounded and non-wounded tissue to ensure that natural barriers on the surface or within the first layer of each tissue are not neglected. 
For the following experiments, a cultivation method for achieving plants with a typical tap root was required. While usually seedlings were pre-sown in multi-pot trays and afterwards transplanted into bigger pots, now the piquing step was circumvented to avoid damage of the roots. Instead, seeds were placed directly into the soil in big pots and the plant roots could develop less restricted by the pot size. This enabled formation of the typical tap root being inoculated as well as the stem in the following experiment. As in the experiment described before, the genotypic effect did not show, and lesions developed equally in both Loras and Zhongyou 821. Although no significant differences were achieved, the root appeared to suffer from visibly shorter lesions in the two genotypes. This trend was not yet visible at 1 and $4 \mathrm{dpi}$ where no difference between the plant tissues could be observed. Only starting at $7 \mathrm{dpi}$, the more slower growing lesions on the root stood out. These results indicate that the root is not resistant to inoculation with S. sclerotiorum. Still, roots are less susceptible compared to the stem, which is the natural tissue of infection of OSR. However, considering the inoculation method of placing a mycelium overgrown agar plug directly on the clean root surface, certain aspects are not taken into account. As an example, environmental effects and the influence of the soil matrix on the pathogen are not attended. Thus, effects of microbes and antagonists in the soil inhibiting myceliogenic germination or plant infection are not regarded (Hu et al., 2014; Troian et al., 2014). Furthermore, it is possible that in the cropping system of OSR, the plants evade myceliogenic infection of the roots. In autumn, when young OSR plants are growing on the field, sclerotia are expected to be dormant (Bolton et al., 2006). In spring, sclerotia have been preconditioned by low winter temperatures in order to trigger apothecia production (Huang \& Kozub, 1991, 1994; Bolton et al., 2006). Thus, preconditioned sclerotia are more likely to perform carpogenic germination. As a consequence, myceliogenic infection of the roots becomes very unlikely and hence it might only occur after mild winters. In addition, mycelial development requires an adequate soil water potential and temperature (Huang \& Kozub, 1994; Hao et al., 2003; Bolton et al., 2006). When at last all conditions favouring myceliogenic germination come into being, new requirements are to be met. To render infection possible, not only host plants need to be present on the field, but also sclerotia need to be located near the roots. This coincidence demands a high density of sclerotia in the soil. Thus, unlike the described greenhouse experiments where root infection was 
forced, roots of winter OSR on the field escape myceliogenic infection due to lack of developmental simultaneousness. This explains the fact that it is rarely observed in nature.

In the greenhouse experiment, root infection was directly aspired and thus successful. Wounding of the root as described in the present experiments considerably facilitated the inoculation. Further investigations should compare the disease development in wounded and non-wounded plant tissues. This would aim to learn whether tissue specific mechanisms are present and were circumvented by injuring before inoculation. Another fact that needs to be considered is the length of the respective plant tissues in stem and root. In the present study, the stem was much longer than the root. This may comprehend longer cells with weaker cell walls and might be a reason of longer lesions in the stem. To gain further knowledge about the influence of length of stem or root on lesion growth, this parameter needs to be included into future studies. This would also allow to calculate correlations between length of stem or root and lesion length.

\subsubsection{Quantification of fungal colonisation}

To find out whether disease severity is related to the actual colonisation of the plant tissue by hyphae, fungal DNA was quantified in healthy and infected plant tissue of stems and roots of Loras and Zhongyou 821 at $7 \mathrm{dpi}$. As expected, no DNA of S. sclerotiorum was found in the uninfected plant material of control plants. From the inoculated plants, the entire lesion has been taken. Areas not being visibly affected by fungal colonisation were removed to avoid dilution of the diseased parts with uninfected material.

Longer lesions developed on the stem compared to the root. According to this, more DNA was detected per gram plant tissue, which is supported by the high correlation between lesion length and amount of DNA. Lesions in the root were shorter and fungal biomass lower compared to the stem. Values of both parameters obtained from the root were not significantly different from the non-inoculated plants, indicating that the roots contained no fungal biomass. Quantification of fungal biomass therefore corroborates the finding of the root being less quickly colonised than the stem.

No significant differences between genotypes Loras and Zhongyou 821 were found, though they are considered as susceptible and partially resistant (Li et al., 1999c). Although this is in accordance with the results for lesion development, former studies showed that 
Zhongyou 821 indeed contained less fungal DNA due to smaller lesions (Höch, 2016). This finding could not be confirmed because of the lacking significant genotypic effect in the present study. Since the experimental procedure - including greenhouse conditions and inoculation method - was similar in both the study by Höch (2016) and the present study, it cannot be explained why Zhongyou 821 was as susceptible as Loras in the latter. Zhongyou 821 is used as partially resistant reference line in many studies not reporting such difficulties (Wang et al., 2004; Zhao et al., 2009; Mei et al., 2011; Ding et al., 2013).

In future experiments, supplementary information about colonisation of plant tissues could be gained by separating different cell tissues from each other, e.g. cortex, vascular tissue, pith and stele. DNA analysis might provide qualitative and quantitative information about the presence of the fungus in these tissues.

\subsection{Infection patterns of S. sclerotiorum in different plant tissues}

Histological studies aimed to reveal the colonisation strategy of hyphae of S. sclerotiorum in the different plant tissues stems, roots and hypocotyls. Previous research shows either histology of tissue from young plants still in the vegetative growth stage (Lumsden \& Dow, 1973; Huang et al., 2008; Davar et al., 2012; Garg et al., 2013; Uloth et al., 2014), or from leaves not being different in vegetative or generative growth (Tariq \& Jeffries, 1984; Jamaux et al., 1995; Williams et al., 2011). These former studies were performed using either light microscopy including suitable staining methods or electron microscopy. There is one report of a successful green fluorescence protein transformation of S. sclerotiorum, allowing detection of hyphal growth in planta via fluorescence microscopy but this study was also conducted on very young plants (de Silva et al., 2009). To the author's knowledge the present study is the first demonstrating S. sclerotiorum growth in flowering oilseed rape plants comparing different plant tissues.

In a first attempt, in vitro grown roots of young OSR seedlings were inoculated and observed under the microscope. The in vitro root test seemingly being a quick and easy method turned out to be unsuitable, since roots of 10-day-old seedlings are too fragile. As the chosen isolate of $S$. sclerotiorum is very aggressive, it quickly covered the small roots with a dense layer of mycelium making it difficult to find distinct infection structures of the fungus. Only single cases delivered a proper view of infection cushions as invasion 
structures. It was impossible to observe whether fungal growth within the plant tissue was taking place. To avoid such an overgrowth by mycelium, a more directed and less forceful inoculation method via macerated mycelium in liquid medium with a defined density of hyphae fragments might be better (Garg et al., 2008).

Since the in vitro experiment was not suited for investigations on fungal growth in different plant tissues, plants were grown until flowering in the greenhouse to obtain circumstances closer to nature. Finally, thin longitudinal sections of the actively growing lesion in the stems, hypocotyls and roots could be observed.

These sections were stained with the Pianese method for observation of plant tissue and fungal hyphae under the light microscope. This dye should stain the plant tissue blueish and fungal cells in purple colour. However, this was not the case in all sections, so the contrast between fungal structures and plant tissue was not always satisfying. Probably the reduction of the $\mathrm{pH}$ in the hyphae surrounding tissue via secretion of oxalic acid (Xu et al., 2015) affects the staining properties. Thus, the different tissues and organisms where not sufficiently distinguishable from each other. Nevertheless, the fungus could be located within the plant tissue. The Pianese stain visualised hyphae mainly growing within the cortex and along the long axis of the stem.

To get a clear differentiation between fungal structures and the plant tissue, sections were stained with Alexa Fluor and propidium iodide and observed with confocal laser scanning microscopy (CLSM). These two stains visualised both subjects of interest, fungus and plant. Nevertheless, it was possible to distinguish them from each other. The CLSM enabled to examine the sections in close detail. The following observations confirm numerous former studies on plant tissue colonisation by S. sclerotiorum. Outside the plant, a mycelial mat was formed above the epidermis, or in a group of hyphae growing along the plant tissue. As a white, fluffy layer on the plant surface, it can also be seen by the naked eye. Multicellular, compound appressoria, called infection cushions, have been described as infection structures of S. sclerotiorum (Lumsden \& Dow, 1973; Hegedus \& Rimmer, 2005; Huang et al., 2008; Davidson et al., 2016; Seifbarghi et al., 2017). In the root but also in the stem, there were new dome-shaped infection cushions formed on the plant surface-apart from the initial inoculation point and the fact that the tissue was already colonised (Jamaux et al., 1995). Infection cushions seemed to exhibit considerable force since they appeared to 
mould the surrounding plant tissue into a slightly convex form. Still, it would be interesting to observe the mycelium during several time steps just before and shortly after inoculation of non-wounded plants to confirm these findings. Regarding the two genotypes, no differences in fungal colonisation were found. This is explained by the identical morphology of stems and roots in both, Loras and Zhongyou 821. Comparing the tissues, however, the cell structure and tissue composition showed clear differences, resulting in a diverse colonisation strategy, as described in the following sections.

After penetration of the stem tissue, the epidermis and cuticle remained clearly visible even near hyphae because they probably were not targeted anymore (Tariq \& Jeffries, 1984). Hyphae were highly vacuolated, indicating the older branching mycelium (Lumsden \& Wergin, 1980; Huang et al., 2008). The branching aimed to enlarge the colonised area. In several cases, plant cells seemed to constitute a barrier for the pathogen, triggering a fanshaped accumulation of hyphae to overcome this barrier (Lumsden \& Dow, 1973). This contributes to the findings of Seifbarghi et al. (2017) who found that pathogenicity genes encoding for cutinases and cellulases were up-regulated already within the first hours of infection, explaining the capability of the pathogen to overcome structural barriers. When reaching the apoplast, hyphae have overcome these barriers, and more space is available for growth. Consequently, hyphae are widening in girth, as has been reported by recent studies (Kabbage et al., 2015; Davidson et al., 2016). This allows the pathogen to establish more volume and, hence, acquire a bigger surface releasing more cell wall degrading enzymes. This fact leads to the following issue. Strongly colonised tissue could not be examined, either because of the strong reflection of laser light by the hyphae covering weaker reflection of plant cells, or rather because plant tissue was simply dissolved by the cell wall degrading enzymes (Tariq \& Jeffries, 1984, 1986; Guimarães \& Stotz, 2004; Bolton et al., 2006; Huang et al. 2008; Seifbarghi et al., 2017). The more hyphae are present, the more cell wall degrading enzymes can be released. Formation of hyphal fronts and disintegration of the plant cells was notably observed in the stem, illuminating the quick spread of the pathogen and the high susceptibility of this plant tissue.

Regarding the stem, a tissue specific colonisation pattern could be observed. Hyphae were found in the cortex, advancing intercellularly and in vertical direction, in parallel along the cells of the stem. This confirmed prior findings of Lumsden \& Dow (1973) and demonstrates 
that the elongated cortex cells with thin cell walls represent a cell structure being very easy to overcome, allowing a quick colonisation. Furthermore, ramifying infection hyphae branching from intracellular penetration hyphae could be found in all tissues, illustrating the aggressiveness of S. sclerotiorum. Mostly intercellular but also intracellular colonisation was observed and is in agreement with different studies (Lumsden \& Dow, 1973; de Silva et al., 2009; Kabbage et al. 2015). These findings corroborate previous reports about hyphae not needing to form specialised infection structures. Instead, causing enzymatic dissolution of structural barriers they advance as a hyphal front (Tariq \& Jeffries, 1984). The coordinated infection front and the orientation of hyphae parallel to the long axis of the stem contribute to macroscopic observations of the quick elongation of lesions along the stem. Therefore, it can be concluded that the presence of hyphae always means the immediate development of a lesion, too.

The typical colonisation pattern revealed in the stem cortex was not observed in the hypocotyl and root. In the roots, hyphae used cell pits - especially those of cells belonging to the ray parenchyma - to spread in the tissue, causing a thickening of single hyphae before penetrating through the cell wall, too (Garg et al., 2013). This strategy contrasted with the pattern observed in the stem and can be explained by the cell morphology of the root tissue: here, cells were more compact and seemed to have thicker cell walls, thus constituting a stronger barrier for the hyphae. Moreover, using the cell pits allowed the fungus to spread also in a horizontal direction. This might explain why lesions elongated less quickly and thus give reasons for the lower susceptibility of the root tissue. It even became visible that hyphae near the stele grew mainly along the ray parenchyma and did not appear in the xylem and xylem parenchyma. Possibly, penetrating cell pits is an energy saving strategy since no or less cell wall degrading enzymes need to be synthesised (Marciano et al, 1983; Xu et al., 2015). Generation of a S. sclerotiorum isolate being deficient in the production of CWDEs could show the importance of these enzymes during colonisation of the root.

In all tissues, hyphae densely colonised the cortex, and they also penetrated the phloem (Lumsden \& Dow, 1973; Huang et al., 2008). The xylem, however, generally remained uninfected. Thus, it seems to be no tissue of special interest for the pathogen, at least not in the advancing zone of hyphae. The pathogen might also be unable to penetrate the 
xylem. Due to the way of taking samples targeting the outer edges of the lesions, only areas containing younger hyphae were observed. Observation of the centre of the lesion which had been infected earlier and, hence, was colonised for a longer period, was not possible. Therefore, it remains unclear whether vascular tissue not being penetrated in the advancing area of younger hyphae would still be infected with established hyphae that can be found at the centre of the lesion (Lumsden \& Dow, 1973; Huang et al., 2008).

Investigations on the leaf also revealed the formation of infection cushions on the plant surface (Jamaux et al., 1995). In contrast to that, other studies report direct penetration of the epidermis by the fungus (Tariq \& Jeffries, 1984; de Silva et al., 2009). On the leaf surface single hyphae chose the way of the least resistance, growing around the stomata instead of crossing them or using them as penetration pathway. This suits to the previously described intercellular growth in the stem, and transition of cell pits in hypocotyl and root. However, the experimental conditions might have stimulated stomatal closure making them useless for fungal penetration (Tariq \& Jeffries, 1984). Inside the leaf tissue, hyphae were rarely observed. Here, again they formed hyphal fronts or chose the way of the least resistance, not penetrating the xylem. In none of the studied tissues any hyphae were found in the xylem vessels, either excluding them as a preferred tissue of the pathogen or indicating that penetration is particularly difficult. Together with observations in stems and roots, this confirms that $S$. sclerotiorum is not a vascular pathogen. Undoubtedly, subsisting on the poor xylem sap would not fit to the life style of this necrotrophic pathogen which possesses the required means to decompose and nourish from the penetrated plant tissue (van Kan, 2006; Horbach et al., 2011; Kabbage et al., 2015).

In regard of the reaction of the plant to fungal penetration, no change could be seen. For example, no thickening of plant cell walls to impede penetration or spread of fungal hyphae was observed. Different stains revealed the formation of defensive structures in OSR after infection with S. sclerotiorum. Those were identified as tyloses via toluidine blue stain. These findings are in accordance with Lumsden \& Dow (1973) who interpreted the crescent-shaped forms as 'crystals'. Tyloses were never found in non-inoculated healthy tissue, mainly in hypocotyl and roots. Occurring exclusively in infected plants later than $1 \mathrm{dpi}$, tyloses only formed in the xylem vessels or xylem parenchyma of all observed tissues. Often, they were found in immediate proximity to hyphae colonising the tissue. Tyloses are 
outgrowths of vessel-associated parenchyma cells and are induced by wounding or pathogen infections with the purpose to block vessels and impair fungal colonisation (Minorsky, 2007; Yadetha \& Thomma, 2013). Formation of tyloses might explain the decelerated lesion growth in the root tissue. However, in this study the vessels were never blocked entirely. Furthermore, since the pathogen is not directly aiming at the xylem, it is questionable why tyloses were formed in the first place and which role they play in symptom development. It is possible that they developed following a general stress reaction of the infected plant. This is likely, because former studies demonstrated that defence mechanisms are initiated, for example altering the cell wall composition by accumulation of lignin or phenolic compounds (Eynck et al., 2009; Sattler \& Funnell-Harris, 2013; Höch, 2016).

Callose is a $\beta-(1,3)$-glucan with antimicrobial properties and cell wall reinforcing functions (Voigt, 2014). The PAS (periodic acid Schiff's) assay was used to stain the component within infected plant tissue. However, no callose became visible. The PAS assay is based on initial oxidation of the carbohydrates to form aldehyde groups and subsequent reaction of those aldehyde groups with the Schiff's reagent (Dr. Michelle Kilcoyne, National University of Ireland Galway; personal communication). Periodic acid (and periodate) oxidises the carbohydrates between two adjacent hydroxyl groups, e.g. the bond between C-2 and C-3 of a glucose. However, some carbohydrates are resistant to oxidation by periodic acid because they do not have two adjacent hydroxyl groups as in $\beta-(1,3)$-linked glucan callose. Because the hydroxyl groups at C-1 and C-3 are occupied by the glycosidic bond, there are no other neighbouring hydroxyl groups available. The hydroxyl groups at C-2 and C-4 do not have an immediate neighbouring hydroxyl group. This explains why callose is PAS negative (Apostolakos et al., 2009). As a consequence, aniline blue was applied as a common method to stain callose (Schenk \& Schikora, 2015; Herburger \& Holzinger, 2016). However, again no callose was visible under the microscope. It is possible that due to secretion of oxalic acid, callose deposition was suppressed (Kim et al., 2008). Still, it cannot be excluded that callose deposition due to pathogen attack is happening (Kim et al., 2011; Kabbage et al., 2013). Probably, due to the paraffin embedding procedure being necessary to obtain thin sections of the tissue, information based on chemical transformations within the tissue gets lost. However, paraffin embedding was needed to stabilise plant tissue being 
macerated by fungal colonisation and thus, was inevitable for the production of thin sections with the microtome.

All in all, according to the similarity in genotypic disease development, no genotypic effect on the colonisation pattern has been found. However, histological studies have shown a clear variability among the colonisation strategies of S. sclerotiorum in different plant tissues.

\subsection{Biochemical responses to the disease}

As a necrotrophic pathogen, S. sclerotiorum causes great damage to the plant tissue. In this process, it is confronted with physical barriers and chemical compounds serving as a defence response. The efficacy of these mechanisms determines whether plants are susceptible or resistant to the pathogen. Among those reactions to infection, accumulation of phenolic acids, peroxidase activity and alteration of glucosinolate patterns were studied and related to their putative role in plant defence of oilseed rape against S. sclerotiorum. Tissue specific reactions were taken into account and related to disease development in the respective tissue, namely stems, hypocotyls or roots.

\subsubsection{Phenolic compounds}

Investigations on phenolic compounds focussed on phenolic acids synthesised during early steps of the phenylpropanoid pathway. These low molecular weight phenols are known to be involved in defence (Vogt, 2010; Ponce de León \& Montesano, 2013). It has been reported that accumulation of phenolic acids may reflect a general activation of host metabolism as initial response to infection (Nicholson \& Hammerschmidt, 1992). Thus, accumulation of particular phenolic acids may indicate a general rise in phenolic synthesis to defend the plant towards infection with S. sclerotiorum.

In the present study, an influence of the infection on phenolic acids could only be observed in single cases. Conjugated and free forms of $t$-cinnamic acid, $p$-coumaric acid, chlorogenic acid, caffeic acid, ferulic acid and sinapic acid, and free salicylic acid were analysed. The free forms were generally increased by inoculation with S. sclerotiorum. This might be explained by the fact that free phenolics are more toxic than their conjugated forms (Lattanzio et al., 2006). Increase of free phenolics after infection was only significant in 
three compounds ( $t$-cinnamic acid, chlorogenic acid, caffeic acid). The increase of phenolic compounds can be explained by the up-regulation of genes that are involved in early phenylpropanoid pathway as found in the susceptible Loras tested in a previous study (Höch, 2016). This has been confirmed by histological studies. However, gene expression alterations occurred only at later infection stages. Various other studies report an increased phenylalanine ammonia-lyase (PAL) activity. PAL is the primary enzyme involved in the phenylpropanoid pathway and is responsible for the production of phenolic compounds (Maher et al., 1994; Prats et al., 2003; Zhao et al., 2007; Ponce de León \& Montesano, 2013). The enzyme therefore bears an important role in pathogen defence. Recent studies contribute to the prior findings reporting the up-regulation of genes involved in the phenylpropanoid pathway after infection, among those the PAL encoding gene (Oliveira et al., 2015; Alvarez et al., 2016). Interestingly, in this study conjugated caffeic, ferulic and sinapic acid showed a decrease after infection but only in the hypocotyl. It is possible that a decreasing concentration hints on defence, assuming that these compounds served as substrate for the synthesis of other chemicals like lignin playing a role in disease resistance (Nicholson \& Hammerschmidt, 1992). Nevertheless, decrease of phenolic acids due to infection is very uncommon compared to other studies (Benko-Iseppon et al., 2003; Dicko et al., 2005; Ponce de León \& Montesano, 2013). Salicylic acid plays an important role in plant defence (Zheng, 2018). Interestingly, no effect on salicylic acids was observed, implementing that it is not of special importance in the present pathosystem. Obtaining gene expression data to add to the biochemical analyses would lead to a more complex view and help understanding the interaction between phenolic acids and infection with S. sclerotiorum.

There was no correlation between resistance of a genotype and accumulation of phenolic acids in this study. In contrast to the results of the present and previous studies, inoculation had no effect on the content soluble phenolic compounds of Helianthus annuus L., but constitutively higher amounts were found in resistant cultivars (Prats et al., 2003). Moreover, resistant genotypes of Sorghum bicolor L. showed higher levels of phenolic compounds without being exposed to biotic stress (Dicko et al., 2005). In addition, a study on Camelina sativa showed genes involved in the phenylpropanoid pathway being downregulated in the susceptible genotype (Eynck et al., 2012). Potentially, the present results 
do not a show genotypic effect on phenolic acids because genotypic differences were lacking.

Apart from salicylic acid, all phenolic acids synthesised from $t$-cinnamic acid showed a plant tissue effect. P-coumaric acid and chlorogenic acid were increased in the hypocotyl and caffeic acid, ferulic acid and sinapic acid were elevated in the stem. The effect was pronounced in the conjugated form and had the same tendency in the free form. Nevertheless, lesion length was not different between the plant tissues and thus, no effect of specific phenolic compounds on lesion growth in stem or hypocotyl tissues was observed. No correlation was found between lesion development and level of phenolic compounds. Nonetheless, there was evidence that changes in regulation of the synthesis of phenolics compounds at the infection site have an inhibitory effect on S. sclerotiorum. Since the exact localisation of phenolic acids has not been investigated, the question remains whether the pathogen gets in contact with phenolic acids.

As precursors of lignin phenolics show indirect inhibition of fungal growth via cell wall strengthening by lignin production (Nicholson \& Hammerschmidt, 1992; Fauld \& Williamson, 1999; Eynck et al., 2012). In the present study, no changes in cell wall composition have been investigated. Whether the increasing content of phenolic acids may be indeed linked to infection by contributing to lignification of plant cell wall cannot be answered. It would be interesting to find out more details, because lignification can serve as mechanical barrier to fungal colonisation and is a general resistance mechanism (Zhao \& Dixon, 2014). Peltier et al. (2009) suggested stem lignin content as a marker for resistance in soy bean selection. Lignified tissue is more resistant to decomposition by cell wall degrading enzymes (Ding et al., 2012). Here, the early response is of major importance. For more investigations, lignin monomers can be visualised by microscopy (Chapple et al., 1992) and quantified by HPLC analysis (Lima et al., 2013). Thus, in following studies lignification should be observed.

It is noteworthy that phenolic compounds may also serve as substrate for the oxidation and therefore detoxification of hydrogen peroxide by peroxidase (Lattanzio et al., 2006; Bartwal et al., 2012). Therefore, peroxidase activity has been analysed and is discussed in the following chapter. 


\subsubsection{Peroxidase activity}

Peroxidase activity was studied in different non-inoculated and inoculated plant tissues of OSR to elucidate its role in resistance against S. sclerotiorum. Peroxidase (POX) is an enzyme activated to reduce cell damage through accumulation of reactive oxygen species (ROS, e.g. superoxide, $\mathrm{H}_{2} \mathrm{O}_{2}$ ) generated by the plant. This accumulation is called the oxidative burst (von Tiedemann, 1997; Zhao et al., 2005). As described above (1.3.1 Phenolics, peroxidase and glucosinolates as part of QDR), programmed cell death due to oxidative burst is an important defence mechanism against biotrophic pathogens which require living cells for nutrition. However, it is unlikely that the strategy of host-derived $\mathrm{H}_{2} \mathrm{O}_{2}$ accumulation plays a role in the present situation with the necrotrophic pathogen S. sclerotiorum. This leads to a second potential function of POX activity: ROS may also be generated and deliberately introduced by the pathogen with the objective to kill host cells. Thereby, S. sclerotiorum like other necrotrophic pathogens regulates ROS. Two NADPH oxidases of the pathogen were identified being important for ROS generation (Kim et al., 2011; Foley et al., 2016). Consequently, the plant tries to stop cell damage by increasing POX activity. In general, POX activity increases upon fungal infection and fast induction of POX activity appears to be advantageous for plant stress response (Passardi et al., 2005; Lattanzio et al., 2006). In the present study, enhanced colonisation of the tissue might be associated with more ROS released by the pathogen and an increase of the POX activity. This is according to the higher lesion lengths in the hypocotyl of Loras accompanied by a higher POX activity. Therefore, POX activity is a measure of the reaction to stress coordinated by the plant and caused by fungal attack, so POX is induced during host plant defence (Almagro et al., 2009).

Examination of POX activity also serves to determine if the plant actively eliminates toxic $\mathrm{H}_{2} \mathrm{O}_{2}$ induced by the pathogen to prevent further cell damage and thus, advancement of the pathogen (von Tiedemann, 1997; Govrin \& Levine, 2000). However, it is questionable if POX activity in OSR is increased quickly enough to activate defence responses inhibiting growth of S. sclerotiorum. Additionally, inoculation had no significant influence on POX activity. In a study on muskmelon (Cucumis melo) infected with the biotrophic pathogen Pseudoperonospora cubensis POX activity was associated with resistance (Reuveni et al., 1992). Reuveni et al. (1992) measured a higher activity in control plants of the resistant line. Moreover, POX activity increased over time in both control and inoculated plants. This 
could also be observed in the present study. Comparing POX activity of inoculated to control muskmelon plants, this led to a lower ratio meaning that inoculation leads to an increase. The increase was shown in both, susceptible and resistant plants, but happened slower in the susceptible one. This has not been shown in OSR infected with S. sclerotiorum, where no consistent results were obtained. Change in POX activity did not hint on a stronger susceptibility or resistance of a specific genotype or plant tissue.

POX does not only play a role as scavenger in redox reactions, but also participates in the biosynthesis of lignin and cross-linking of phenolic compounds. Lignin is important in plant defence as it limits expansion of infection through the establishment of structural barriers (Asada, 1992; O'Brien et al., 2012). The cross-linking of monolignol monomers is catalysed by POX using $\mathrm{H}_{2} \mathrm{O}_{2}$. POX mediates the $\mathrm{H}_{2} \mathrm{O}_{2}$-dependant oxidative linking of monolignols such as p-coumaryl, coniferyl and sinapyl alcohols to lignin, resulting in cell wall rigidification (Ros Barceló et al., 2007; O’Brien et al., 2012). It remains unclear whether these mechanisms are activated fast enough during infection with S. sclerotiorum. The pathogen may already penetrate plant tissue within a few hours after infection (Andrew et al., 2012; Seifbarghi et al., 2017) and spread considerably in the tissue in one day (Davidson et al., 2016; Höch, 2016; this study). Therefore, increase in POX activity would be necessary within the first 24 hours, which has not been recorded in the experiments. Hence, it is unlikely that cell wall enhancement is happening fast enough to provide protection against colonisation by this aggressive pathogen. In conclusion, the present study provides little evidence that lignification plays an important role as a resistance factor in oilseed rape against S. sclerotiorum.

\subsubsection{Glucosinolates}

Several studies have demonstrated that the major function of glucosinolates (GSL) is to defend plants against herbivores and pathogens (Mithen, 1992; Zhao \& Meng, 2003b; Rostas et al., 2003; Bednarek et al., 2009; Buxdorf et al., 2013; Frerigmann et al., 2016). Therefore, investigations on Glucosinolates were made to elucidate their role in defence against S. sclerotiorum in respect to different plant tissues of OSR. In Brassicaceae, GSL are secondary metabolites produced in main plant tissues such as leaves and transported to the seeds (Chen et al., 2001; Feng et al., 2012). Since GSL have different amino acids as precursors, they can be classified into aliphatic, aromatic and indole GSL (Mithen, 1992; 
Bartlet et al., 1999; Halkier \& Gershenzon, 2006). GSL are influenced by different factors like environment, genetics, developmental stage and abiotic or biotic stresses. In the following discussion, these factors will be referred to in order to explain and understand the results described above.

In this study, results from several experiments varied considerably. Although they were executed in a controlled environment, the amount of GSL differed depending on temperature, light and season. Levels and composition of GSL strongly reflect environmental factors (Fahey et al., 2001; Halkier \& Gershenzon, 2006), which could not be totally excluded in the greenhouse.

GSL are influenced by genetics and hence by Brassica species and also by the genotype (Fahey et al., 2001). Compared to B. oleracea and B. rapa, B. napus contains relatively low levels of GSL, possibly explaining its generally lower resistance against S. sclerotiorum. Loras is a double-low and Zhongyou 821 a double-high oilseed rape variety (Li et al., 1999c; Li et al., 2001; Wang et al., 2004). This classification refers to the seed GSL content and it is known that seed and leaf GSL concentrations are under separate genetic control (Mithen, 1992). However, since GSL are also transported from vegetative to generative plant parts, there probably is a relationship between both. This might also explain why the partially resistant genotype Zhongyou 821 contained higher GSL amounts than the susceptible genotype Loras. It might also be the reason for higher amounts of GSL found in all studied plant tissues of Zhongyou 821 in contrast to Loras.

In view of the antimicrobial role of GSL, one might think that higher amounts lead to inhibition of fungal growth. However, lesion length and total GSL content were not correlated: Zhongyou 821 showed higher GSL content than Loras but exhibited the same lesion extend. Fan et al. (2008) reported longer lesion length in susceptible, double-low varieties compared to both, double-low and double-high resistant lines, indicating that GSL content does not contribute to resistance. Therefore, resistance levels are not generally elevated by a higher GSL content (Giamoustaris \& Mithen, 1997; Zhao \& Meng, 2003b). This is true for vegetative plant tissues as well as seeds (Li et al. 1999b; Fan et al., 2008). To gain further knowledge, GSL content was analysed in different genotypes. 


\section{Glucosinolate content and resistance of different genotypes are not correlated}

The OSR genotype Zhongyou 821 is reported to be partially resistant against S. sclerotiorum. As described above, it also contains higher GSL contents compared to the susceptible genotype Loras. In this study disease development after inoculation with S. sclerotiorum was equal in both genotypes, the partially resistant Zhongyou 821 and the susceptible Loras. Nonetheless, an attempt was undertaken to relate GSL amounts in different plant tissues to resistance by comparing the responses of different genotypes to the pathogen.

Genotypic variability in terms of susceptibility to S. sclerotiorum and GSL content in stem and root has been investigated. Samples from infected stems and non-inoculated stems and roots were taken for GSL quantification. As expected from previous findings of this study, non-inoculated stems contained far less amounts than roots. Stem-GSL content rose after inoculation being mainly to be attributed to a rise in indole GSL. The higher amounts of GSL in some genotypes were not accompanied by restriction of fungal growth and, hence, reduced lesion length. This confirms previous findings of the present and other studies. Therefore, enhanced resistance due to higher total GSL amount has been disproven in this study as already done so by other authors (Giamoustaris \& Mithen, 1997; Li et al. 1999b; Zhao \& Meng, 2003b; Fan et al., 2008).

The attribute 'double-low' or 'double-high' variety for the seed erucic acid and glucosinolate content did not play a role referring to stem and root GSL amount. Most of the tested genotypes are characterised as double-low cultivars. Only Zhongyou 821 and Furax are reported to contain high GSL contents (Li et al., 1999c; Zhao et al., 2004). This characterisation refers to the seeds and is not genetically related to the GLS amount in other plant tissues (Li et al., 1999a; Kliebenstein et al., 2001; Feng et al., 2012). However, Furax showed extraordinarily high amounts in the root. In contrast, Zhongyou 821 did not display higher GSL contents compared to the other genotypes and was not significantly different to Loras, although the presence of higher GSL amounts in Zhongyou 821 has been the case in previous experiments. This can be only explained with GSL being very sensible to environmental impact (Fahey et al., 2001; Halkier \& Gershenzon, 2006).

As mentioned before, GSL are influenced by the developmental stage of plants (ClossaisBesnard \& Larher, 1991; Fieldsend \& Milford, 1994; Brown et al., 2003). During vegetative growth, GSL content in the plant is relatively high and serves as a reliable protection against 
feeding insects. With the onset of flowering, GSL concentration in the plant tissue decreases. Interestingly, this is exactly the developmental stage when S. sclerotiorum infects the plant. Moreover, flowering time and resistance are negatively correlated, as described above (4.1 Lack of complete genotypic resistance against S. sclerotiorum). Ascospores of $S$. sclerotiorum emerge in coincidence with the flowering oilseed rape plants. After infection, loss of cellular integrity as a consequence of cell damage initiates GSL breakdown by myrosinase-catalysed hydrolysis of the thioglucoside linkage. The breakdown products have beneficial effects by enhancing plant protection. However, this effect does not matter in certain pest or disease interactions. It is possible that during coexistence with the host, not only feeding insects but also fungal pathogens have adapted to specific GSL (Giamoustaris \& Mithen, 1997; Fan et al., 2008; Buxdorf et al., 2013). While host plant protection is one of the key roles of secondary metabolites such as GSL, this function is simply overcome by fungal necrotrophs. This is notably imaginable regarding the fact that necrotrophic pathogens can detoxify secondary metabolites (Osbourn, 1996; Bednarek et al., 2009) and might explain the lacking correlation between GSL content and lesion length in this study. Consequently, comparison of different OSR genotypes shows that high amounts of total GSL do not enhance resistance against fungal pathogens.

\section{Specific glucosinolates are influenced differently by genotype, plant tissue and inoculation}

These findings leading to the conclusion that the total GSL content does enhance resistance prompt a more careful consideration of single GSL components or classes to be studied with regard to biotic stresses in different tissues (Bennet \& Wallsgrove, 1994). GSL identified in the present study confirm previous findings in OSR of the same aromatic (gluconasturtiin) and indole GSL (glucobrassicin, neoglucobrassicin, 4-methoxyglucobrassicin) and various aliphatic GSL (Li et al., 1999a; Rostas et al., 2002). Among the identified specific GSL, a distinct pattern was observed. Accordingly, GSL were grouped depending on the metabolic pathway and their responses to inoculation in particular plant tissues (Fig. 52). Aliphatic GSL are synthesised by transformation of methionine, followed by side chain elongation. Those synthesised at the beginning of the pathway showed higher concentrations in the root compared to the stem (glucoiberverin, glucoerucin, gluco- 
berteroin; marked yellow). Glucoraphanin, synthesised subsequently in the pathway, showed the same tendency. Other alipathic GSL formed in the later steps of the pathway were higher in the stem than in the root (glucoiberin, gluconapin, glucoalyssin and glucobrassicanapin; marked green and blue). This was also true for the only aromatic GSL, gluconasturtiin. All indole GSL especially showed reaction to the infection (glucobrassicin, 4-methoxyglucobrassicin, neoglucobrassicin; marked red).

Referring to aliphatic GSL this pattern indicates that formation of specific GSL takes place in different plant tissues. The observations lead to the assumption that after the first step of the pathway taking place in the root, glucoiberverin, glucoerucin and glucoberteroin are transformed only slowly into subsequent compounds (Fig. 53, see A., indicated in orange). Consequently, these initially synthesised GSL accumulate in the root and hence show higher concentrations than the subsequently formed compounds. The contrary is happening in the stem (Fig. 53, see A., indicated in blue). Here, GSL synthesised primary in the pathway are modified immediately, transforming them according to the following steps of the pathway. Consequently, in the stem glucoiberin, gluconapin, glucoalyssin and glucobrassicanapin are higher than in the root. Another possibility not necessarily excluding the first is that more GLS are initially synthesised in the root (Fig. 53, see B., indicated in orange). This also corresponds to the generally higher amounts of GSL in the root than in the stem. The compounds (glucoiberverin, glucoerucin and glucoberteroin) are then modified according to the pathway into glucoiberin, gluconapin, glucoalyssin and glucobrassicanapin. They are directly transported into the stem where they accumulate (Fig. 53 , see B., indicated in blue).

In conclusion, GSL concentration in different tissues seems to underly tissue specific QTL (Kliebenstein et al., 2001; Feng et al., 2012). Brown et al. (2003) detected indole GSL as the major class in the root, and aliphatic GSL were highly abundant in photosynthetic tissue. However, main GSL classes in the plant tissues depend on the developmental stage (Clossais-Besnard \& Larher, 1991) explaining why in this study the root generally contained higher GSL amounts than the stem.

Pathogen infection had no or only minor effects on total GSL levels. This has already been found by Stotz et al. (2011) and implies a putative role of GSL in constitutive resistance. Among the GSL compounds analysed, only indole glucosinolates showed an increase after 
inoculation (Fig. 52; marked red). Defence capacity of GSL is depending on the side chain determining the class of the compound. Antifungal activity can especially be attributed to indole GSL (Schlaeppi et al., 2010; Sanchez-Vallet et al., 2010; Stotz et al., 2011). This has already been revealed by Mithen et al. (1986) by showing inhibited growth of Leptosphaeria maculans in the presence of glucobrassicin in vitro and Rostas et al. (2003) who demonstrated that infection with Alternaria brassicae leads to increased levels of indole GSL. Gluconasturtiin, glucobrassicin and neoglucobrassicin are only present in vegetative tissues (Clossais-Besnard \& Larher, 1991). This underlines their function in plant defence because in OSR, vegetative tissues are the aim of fungal pathogens. Furthermore, Zhao \& Meng (2003b) found a QTL being responsible for glucobrassicin content and is presumably linked with resistance to $S$. sclerotiorum infection on the stem of maturing plants. In addition, their study showed that QTL for glucobrassicin and resistance overlap accounting for its strong reaction after inoculation. Moreover, mutant plant lines deficient in indole or aliphatic GSL biosynthesis were hypersusceptible to S. sclerotiorum (Stotz et al., 2011). Many studies on the model plant Arabidopsis thaliana demonstrated the pathogeninduced activation of genes linked with tryptophan metabolism and thus indole GSL synthesis (Clay et al., 2009; Sanchez-Vallet et al., 2010; Frerigmann et al., 2016). Aside from the present study, these findings reveal that among the different classes of GLS, notably indole GSL are involved in plant defence. 


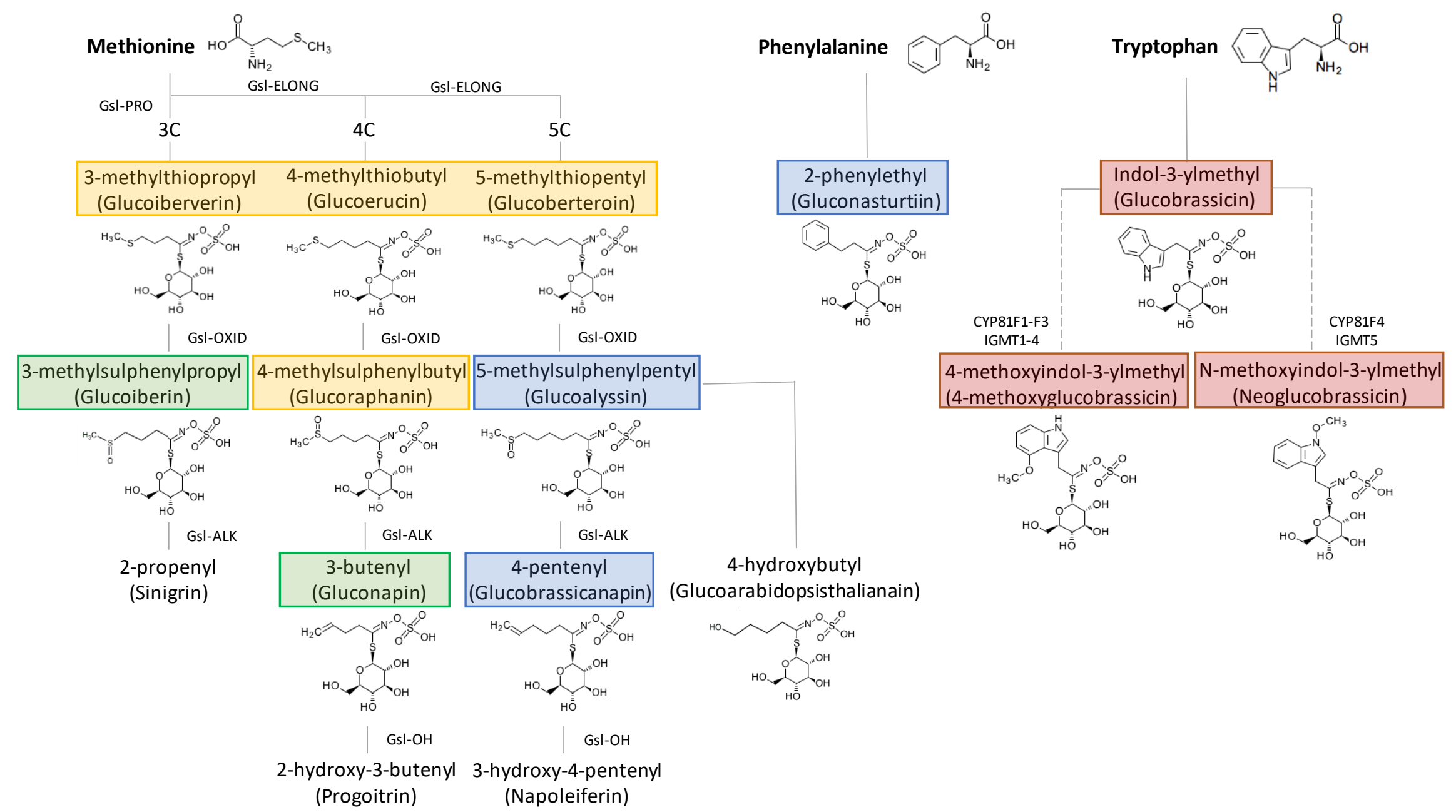

Fig. 52 Aliphatic, aromatic, and indole glucosinolates (GSL) deriving from the amino acids methionine, phenylalanine and tryptophan, respectively. Pathway including the genes controlling elongation and side chain modifications as far as known. GSL were measured in non-inoculated and S. sclerotiorum inoculated plant tissues stem and root of the oilseed rape genotypes Loras and Zhongyou 821. Colours indicate reaction of GSL content to experimental factors in this study. Yellow - GSL content in root higher than stem, blue - GSL content in root higher than stem only at $7 \mathrm{dpi}$, green - GSL content in stem higher than root, red - increase due to infection (after Sønderby et al., 2010; Sotelo et al., 2014; Pfalz et al., 2016). 
A.

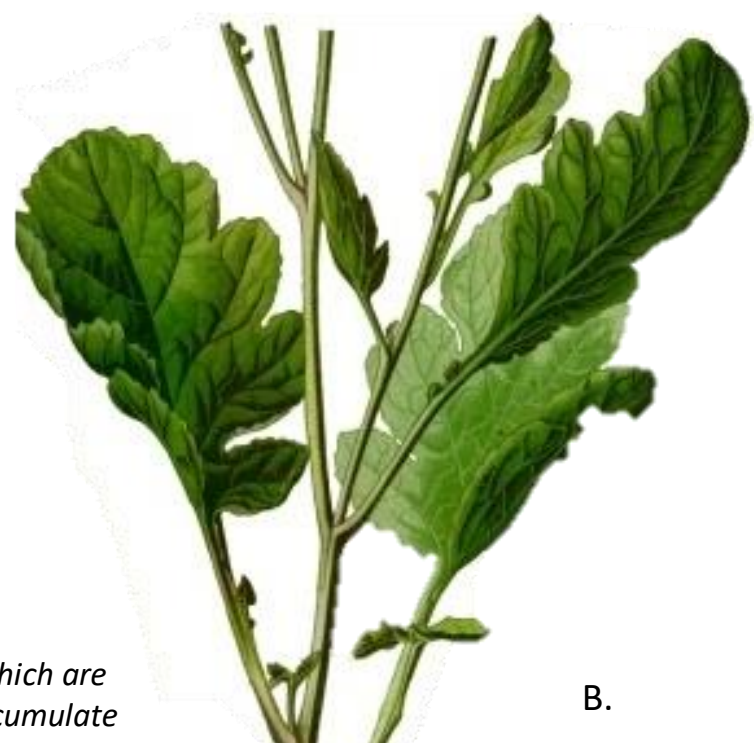

Synthesis of primary GSL which are transformed quickly and accumulate

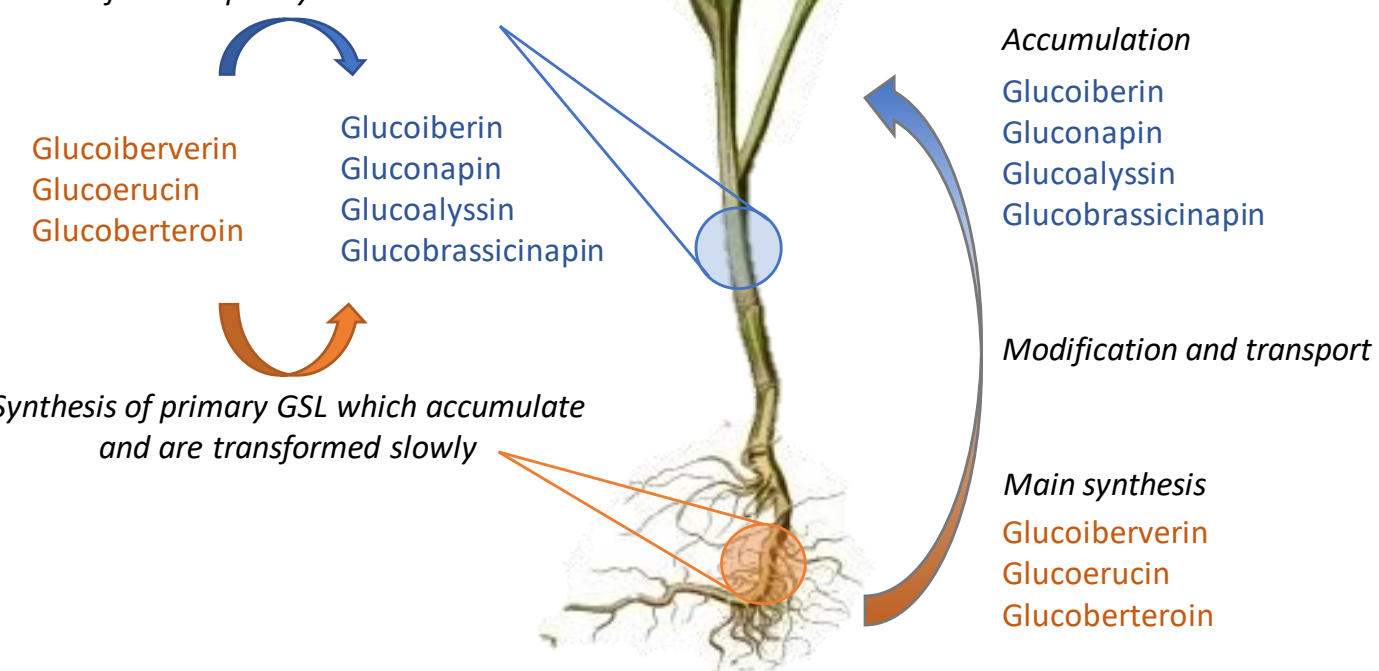

Fig. 53 Sources, transfer and transformation of specific aliphatic glucosinolates (GSL) accumulating in roots (early pathway synthesis, marked orange) or stems (later pathway synthesis, marked blue) of oilseed rape A: Equal synthesis of primary synthesised GSL in both stem and root with either quick (stem) or slow (root) transformation into subsequent GSL B: Main synthesis of primary GSL in the root with modification to subsequent GSL and immediate transport into the stem. Arrows show downstream compound formation (plant image: Wikipedia).

\subsection{Effect of growth regulators on development of plants and disease}

Previous studies had shown that disease response of oilseed rape to S. sclerotiorum is depending on the stem morphology (Höch, 2016). Not only the plant height, but also the cell lengths of the stem might be related to resistance. Moreover, the stability of the tissue and accumulation of phenolic compounds and lignin in the cell walls are important determining factors for the spread of the fungus. In this context, the effect of growth regulators on the resistance of OSR to infection with S. sclerotiorum is an interesting issue. 
Different agrochemicals have been tested for their influence on the plant phenotype and, hence, on the extent of $S$. sclerotiorum infection. Three of these products have both growth regulatory as well as fungicidal properties. In agricultural practice, they are applied in autumn to ensure optimal development of oilseed rape before winter. In spring, a second application prevents the crop from lodging.

The autumn application is aimed to keep plants in rosette stage to prevent shoot elongation and thus protect them against frost damage. In spring, however, plants take up shoot elongation to allow normal development and harvesting in time. Consequently, application of the products on the two oilseed rape genotypes Loras and Zhongyou 821 had no effect on the developmental stage. Nevertheless, growth regulators alter the plant morphology in a long-lasting way. This could be seen in the results where application of all products led to a significantly reduced plant height. However, the stem diameter was not affected. This is caused by inhibition of gibberellin synthesis, the mode of action of all active agents being applied in this study. Gibberellin is a phytohormone promoting cell elongation (Child et al., 1993; Gaspar et al., 1996; Rademacher, 2000). When the biosynthesis of gibberellin is down-regulated, reduced cell enlargement leads to more compact cells and smaller plants (Aloni et al., 1990). In general, growth regulatory effects on plant height were correlated with lesion length. This might be one reason why in Zhongyou 821 the treatment also led to reduced lesion length caused by S. sclerotiorum: the fungus encountered cell wall barriers more frequently and hence the colonisation progress was restricted. Furthermore, shorter cells are expected to have thicker cell walls. These findings corroborate the explanation of a tissue specific disease development and colonisation strategy (chapter 4.3.1 and 4.4). By applying stem shortening growth regulators, the tissue specific effect observed in the roots can be simulated. This effect is related to the tissue specific morphology. The root being the shorter tissue with more compact cells with stronger cell walls compared to the stem also performed smaller lesions. Consequently, the stem of a non-treated plant probably is constructed of longer cells with thinner cell walls compared to a shortened stem of a GR-treated plant with compact cells being difficult to be overcome by a pathogen. This might explain the reduced lesion growth in treated stem tissue.

However, not all products were capable to protect the plant efficiently: although the plant height is reduced as strong as in Moddus and Caramba, treatment with Carax and Toprex 
did not lead to a significant reduction of lesion growth. Like Caramba, Carax contains metconazole. This compound inhibits the ergosterol synthesis. Ergosterol is present in fungal cell membranes to maintain their structure and function, and the application leads to impeded cell wall synthesis of pathogens (Rademacher, 2000). This mode of actions seems to be less affective on S. sclerotiorum. Concerning Toprex, the two triazoles may affect other pathogens like Leptosphaeria spp. (Zamani-Noor \& Knüfer, 2018), but cannot control infection by S. sclerotiorum.

Whatever the effect of growth regulators and fungicides on S. sclerotiorum is, it has to be kept in mind that oilseed rape is able to compensate low disease pressure (del Río et al., 2007) and thus, chemical crop protection measures are not always economically efficient (Dunker, 2005). In this study, not only the effect on the pathogen itself was of interest but also on the plant phenotype. No histological investigations or biochemical studies were executed. Therefore, it is not clear weather growth regulators lead to a cell morphology or cell wall composition that impedes infection. Examination of thin sections of the stem might show in detail the effect of growth regulators on cell morphology in terms of length of the cells and size of the cell wall. Analyses of cell wall composition regarding phenolic acids and lignin could explain the effect of growth regulators on resistance. Understanding changings in cell wall composition and plant tissue morphology might help to elucidate protective plant properties that can be addressed in future breeding strategies. 


\section{Summary}

Oilseed rape is an important oil crop and is intensively cultivated in Europe. In the field, plants can be infected by Sclerotinia sclerotiorum, the causal agent of sclerotinia stem rot. S. sclerotiorum is a necrotrophic fungus. In spring, sclerotia give rise to apothecia which release ascospores into the air. Ascospores land on the petals of flowering oilseed rape plants. The infected petals fall down and stay attached on leaves and leaf axils of the plant. From there, the infection spreads into the stem and causes severe damage of the plant tissue. The consequences are rotten stems, lodging plants and early shattering pods. Under favourable conditions for the pathogen, this can lead to high yield losses.

Currently no sources for genotypic resistance are known. To identify resistant candidates, disease assessment was executed under controlled conditions on a broad set of Brassica napus genotypes belonging to different crop types. Zhongyou 821 and Loras were chosen as partially resistant and susceptible reference genotype, respectively. Inoculation of the superficially injured stem of flowering plants with a mycelium overgrown agar plug was followed by the screening of lesion length and disease score seven days post inoculation. 183 accessions were assessed, revealing intermediate to strong symptom development in more than half of the candidates. None was completely resistant. Genotypes with assessment values lower than Zhongyou 821 existed but were not significantly different. Generally, reference lines did not perform consistent responses to inoculation. Furthermore, not all tested lines developed the required phenotype with an elongated stem reaching of flowering stage. Therefore, it is critical whether the achieved data are representative. However, knowledge about lack of genotypes harbouring resistance confirms former studies. As a consequence for this study, other sources for resistance against sclerotinia stem rot were sought by focussing on tissue specific disease responses.

As the name declares, the disease caused by $S$. sclerotiorum affects the stem of OSR. However, little is known about the roots. Considering the fact that S. sclerotiorum survives as sclerotia in the soil, the infection of the roots could be regarded as self-evident. However, root infection is rarely observed in nature. Therefore, in-depth studies on tissue specificity were performed on Loras and Zhongyou 821, known to perform contrasting responses to inoculation with S. sclerotiorum. 
In the following, the root was inoculated as well as the stem. It could be shown that under favourable conditions the pathogen successfully infects the root tissue. Nevertheless, lesion expansion is far slower compared to the stem tissue, being explained by the comparable shortness of this plant tissue. Another possible reason is the tissue related morphology, causing a specific colonisation pattern. Histological studies of longitudinal sections showed hyphae within the cortex growing along the stem axis. The cortex has elongated cells with thin cell walls. The fungus rarely colonised the phloem and was not found in the xylem or the pith. In contrast to that, hyphae within the root tissue not only grew in vertical but also horizontal direction using cell pits as an easy way of passage. Thus, while in the stem the direction of lesion growth is in a strictly directed longitudinal way, in the root it is more omnidirectional being reflected by the decelerated lesion development. No genotypic effect was visible in histological studies. As a concluding remark, tissue specific resistance could partly be proven based on symptom development.

Biochemical responses to infection were analysed in terms of the effect of genotype and plant tissue.

Phenolic acids synthesised at the beginning of the phenylpropanoid pathway were quantified. No distinct pattern related to the respective plant tissues was recognised, and no influence of phenolic acids on the development of the lesion could be observed. Therefore, the responses could not clearly be attributed to defence mechanisms. Former studies show correlation between constitutively higher concentrations of phenolic compounds and resistance. However, no genotypic effect could be shown in this study caused by the lack of differences in disease development between the tested genotypes. Biochemical data need to be completed by gene expression studies to enlighten the interaction between phenolic acids and infection with S. sclerotiorum with respect to tissue specificity.

The role of peroxidase in response to infection was investigated. In the pathosystem of S. sclerotiorum, POX probably serves as scavenger of reactive oxygen species released by the pathogen. However, results of different experiments were not consistent. Therefore, it cannot be said that POX activity changes due to infection. POX did neither show an effect related to genotypic resistance nor to tissue specific plant defence responses. With regard 
to its potential role in cell wall rigidification, it is doubtful that POX is involved in an effective protection against the aggressive pathogen S. sclerotiorum.

In this study, glucosinolates being representative for vegetative tissue of OSR were identified. GSL are known to play a role against herbivores and pathogens. However, a high total GSL amount does not result in higher resistance against infection with S. sclerotiorum. In fact, only observation of specific classes of GSL provides answers about the role of GSL in resistance. GSL can be classified in aliphatic, aromatic and indole GSL depending on the amino acid precursor. The biosynthetic pathway of aliphatic GSL might be related to the amount of the specific compounds in different plant tissues. Therefore, primary synthesised compounds are more present in the root while the concentration of subsequent compounds is higher in the stem. Obviously, the first steps of the pathway take place in the root while the following steps are located in the stem. However, aliphatic GSL were not related to resistance. In contrast, indole GSL showed a strong increase due to infection. They are known to be more present in the root, explaining the reduced susceptibility of this plant tissue. Their role in plant defence has already been shown by QTL mapping identifying overlapping loci of resistance and indole GSL synthesis. Taking all findings into account, tissue specific GSL pattern could be demonstrated.

The beneficial effect of growth regulators on plant health could be correlated to their effect on plant height, resulting in a better tissue structure with compact cells. This can also be seen in the root tissue compared to the stem. It can be assumed that application of growth regulators enhances the cell morphology and cell wall composition towards infection with S. sclerotiorum. Still, this remains to be clarified in further studies.

The results obtained from this study are another step towards identification and characterisation of resistance mechanisms in oilseed rape against S. sclerotiorum with special regard to tissue specific properties. All in all, further investigations including gene expression analysis are required to elucidate the role of phenolic acids, peroxidase activity and glucosinolates in defence response against S. sclerotiorum. Moreover, histological revelation of the colonisation pattern should be completed with histochemical studies to gain a more complex picture linked to the plant's response. Aside from studies on genotypic and tissue specific resistance on oilseed rape and related species, more investigations should be made on how to prevent sclerotinia stem rot in agricultural praxis. 


\section{Zusammenfassung}

Raps ist eine bedeutende Ölfrucht, die in Deutschland und Europa intensiv kultiviert wird. Dies führt zur Vermehrung von Krankheitserregern wie Sclerotinia sclerotiorum, dem Verursacher der Weißstängeligkeit. S. sclerotiorum ist ein nekrotrophes Pathogen. Im Frühling bilden die Dauerkörper Apothecien aus, welche Ascosporen in die Luft schleudern. Die Ascosporen infizieren über hinunterfallende Blütenblätter des Rapses schließlich den Stängel, wo durch den Befall starke Schäden verursacht werden. Das zerstörte Stängelgewebe hat lagernde Pflanzen und vorzeitiges Aufplatzen der Schoten zufolge. Dies kann zu hohen Ertragsverlusten führen.

Aktuell sind keinerlei Rapssorten mit Resistenz gegen S. sclerotiorum bekannt. Daher wurden verschiedene Genotypen, die der Spezies Brassica napus angehören, unter kontrollierten Bedingungen auf Anfälligkeit getestet. Dabei dienten Zhongyou 821 und Loras als partiell resistente bzw. anfällige Referenz. Die Inokulation erfolgte mittels eines mycelüberzogenen Agarscheibchens, welches am oberflächlich verletzten Stängel befestigt wurde. Sieben Tage später erfolgte die Bonitur. Von den 183 geprüften Akzessionen erwies sich die Mehrzahl als mittel bis stark anfällig. Vollständig resistente Genotypen traten nicht auf. Zwar gab es Genotypen, deren Boniturwerte unterhalb von Zhongyou 821 lagen, jedoch nicht signifikant unterschiedlich waren. Überdies zeigten die Referenzgenotypen keine konsistente Symptomausprägung. Außerdem entwickelten nicht alle Akzession den für die Inokulation erforderlichen Stängel und erreichten nicht das Blütenstadium. Dementsprechend kann nicht sichergestellt werden, dass die Daten repräsentativ und reproduzierbar sind. Nichtsdestotrotz wurde das Fehlen resistenter Sorten bereits in anderen Studien festgestellt, und ist somit bestätigt worden. Im Folgenden sollten andere Quellen für Resistenz identifiziert werden, welche auf gewebespezifischer Befallsreaktion beruhen. Weißstängeligkeit betrifft in der Regel den Rapsstängel. Der Befall von Wurzeln erfolgt üblicherweise nicht. Um die Hintergründe dieses Umstandes zu ergründen, wurden Studien zu gewebespezifischer Resistenz an den kontrastierenden Genotypen Loras und Zhongyou 821 durchgeführt. Sie ergaben, dass das Wurzelgewebe unter den gegebenen Bedingungen durchaus von S. sclerotiorum befallen wird und sich Läsionen ausbilden. Diese entwickelten sich jedoch bedeutend langsamer als im Stängelgewebe. Ein Grund dafür 
könnte sein, dass die Wurzel selbst vergleichsweise kurz ist. Ein weiterer Grund könnte in der Zellmorphologie liegen, welche wiederum ein gewebespezifisches Kolonisierungsmuster bedingt. Histologische Untersuchungen zeigten, dass die Pilzhyphen parallel zur Stängelachse innerhalb des Cortex wachsen, welcher lange, dünnwandige Zellen aufweist. Im Wurzelgewebe hingegen wuchsen die Hyphen nicht nur in vertikaler, sondern auch horizontaler Richtung indem sie Zellwandtüpfel nutzten, um sich effizient im Wurzelgewebe verbreiten zu können. Dies erklärt möglicherweise, warum das Läsionswachstum weniger stark in Längsrichtung verläuft als im Stängel. Während das Phloem gelegentlich befallen wurde, blieben Xylem und Stängelmark frei. Zudem gab es keinen genotypischen Effekt. Insgesamt konnten die histologischen Beobachtungen teilweise die gewebespezifische Resistenz der Wurzel aufzeigen.

Befallsreaktionen auf biochemischer Basis wurden mit Blick auf genotypische und gewebespezifische Effekte analysiert. Dazu wurden zum einen Phenolsäuren, welche zu Beginn des Phenylpropanoidsyntheseweges gebildet werden, quantifiziert. Es konnte kein Zusammenhang zur Gewebespezifität festgestellt werden. Die Inokulation hatte lediglich einen Einfluss auf die freien Phenole, was derzeit nicht begründet werden kann. Gleichzeitig konnte umgekehrt keine Wirkung von Phenolen auf die Läsionsentwicklung gezeigt werden. Daher ist Phenolen keine eindeutige Bedeutung als Schutzmechanismus gegenüber S. sclerotiorum nachzuweisen. Ebensowenig wurden genotypische Effekte sichtbar, was an dem fehlenden Unterschied in der Läsionsausprägung der getesteten Genotypen liegt. Diese Ergebnisse sollten zukünftig durch Genexpressionsstudien vervollständigt werden, um die Interaktion zwischen Phenolsäuren und der Infektion mit S. sclerotiorum mit Bezug auf die Gewebespezifität besser verstehen zu können.

Des Weiteren wurde die Rolle der Peroxidase bei der Befallsreaktion untersucht. Es ist anzunehmen, dass POX reaktive Sauerstoffspezies neutralisiert, welche von S. sclerotiorum in das Pflanzengewebe entlassen werden. Die Ergebnisse dieser Studie waren jedoch nicht konsistent. Somit kann keine klare Befallsreaktion in Form einer zu- oder abnehmenden POX-Aktivität aufgezeigt werden. Auch zeigte sich kein Effekt durch Genotyp oder Pflanzengewebe. Im Zusammenhang der Zellwandverfestigung ist es unwahrscheinlich, dass dieser Mechanismus bei Befall durch S. sclerotiorum ausreichend schnell und effektiv wirkt. 
Zu guter Letzt wurden Glucosinolate analysiert. Im vegetativen Pflanzengewebe wurden repräsentative GSL identifiziert. Obwohl bekannt ist, dass GSL Schutz gegen Schädlinge und Krankheitserreger bieten, kann einem hohen GSL Gehalt nicht automatisch eine höhere Resistenz des jeweiligen Gewebes oder Genotyps gegen S. sclerotiorum zugesprochen werden. Vielmehr müssen spezifische Einzelkomponenten betrachtet werden. GSL können entsprechend der Aminosäure, von der sie abstammen, in aliphatische, aromatische und indolische GSL klassifiziert werden. Der Syntheseweg der aliphatischen GSL zeigt gewebespezifische Muster. So scheinen die anfänglichen Schritte in der Wurzel abzulaufen, die darauffolgenden jedoch im Stängel, was anhand der Gehalte der jeweiligen Komponenten festzustellen war. Jedoch reagierten einzig die indolischen GSL in Form einer starken Zunahme auf Inokulation. Ihr hohes Vorkommen in der Wurzel könnte neben der Zellmorphologie ein weiterer Grund für die reduzierte Anfälligkeit dieses Gewebes sein. Insgesamt konnte die Verteilung von GSL klar der Gewebespezifität zugeordnet werden.

Die positive Wirkung von Wachstumsregulatoren auf die Pflanzengesundheit konnte mit dem Effekt der reduzierten Pflanzenhöhe und damit zusammenhängender Zellmorphologie begründet werden. Kürzere Pflanzen besitzen möglicherweise kleinere, kompaktere Zellen mit verstärkten Zellwänden, die weniger schnell kolonisiert werden. Ein ähnlicher Effekt konnte im Wurzelgewebe beobachtet werden, welches eine kompaktere Zellmorphologie aufweist als das Stängelgewebe. Dies könnte zukünftig gegebenenfalls durch histologische Studien bestätigt werden.

Die Erkenntnisse der vorliegenden Studie sind unter besonderer Beachtung gewebespezifischer Gegebenheiten ein weiterer Schritt zur Identifizierung und Charakterisierung von Resistenzmechanismen in Raps gegen S. sclerotiorum. Obwohl biochemische Untersuchungen Antworten liefern konnten, sollte die Rolle einiger Aspekte durch Genexpressionsstudien noch klarer definiert werden. Die erfolgten histologischen Untersuchungen können durch histochemische Ansätze noch vertieft werden, um so ein detaillierteres Bild der Befallsreaktion zeichnen zu können. Neben der Forschung zu genotypischer und gewebespezifischer Resistenz, bleibt die Prävention von Weißstängeligkeit und der Schutz vor Befall in der landwirtschaftlichen Praxis eine bestehende Herausforderung. 


\section{References}

Abawi GS, R Provvidenti, DC Crosier, JE Hunter (1978). Inheritance of resistance to white mold disease in Phaseolus coccineus. Journal of Heredity 69 (3), 200-202.

Abawi GS, RG Grogan (1979). Epidemiology of diseases caused by Sclerotinia species. Phytopathology 69 (8), 899-904.

Adams PB, WA Ayers (1979). Ecology of Sclerotinia species. Phytopathology 69 (8), 896899.

Almagro L, LV Gómez Ros, S Belchi-Navarro, R Bru, A Ros Barceló, MA Pedreño (2009). Class III peroxidases in plant defence reactions. Journal of Experimental Botany 60 (2), 377390.

Aloni R, MT Tollier, B Monties (1990). The role of auxin and gibberellin in controlling lignin formation in primary phloem fibers and in xylem of Coleus blumei stems. Plant Physiology 94, 1743-1747.

Alvarez A, M Montesano, E Schmelz, I Ponce de León (2016). Activation of shikimate, phenylpropanoid, oxylipins, and auxin pathways in Pectobacterium carotovorum elicitors-treated moss. Frontiers in Plant Science 7, 328.

Andrew M, R Barua, SM Short, LM Kohn (2012). Evidence for a common toolbox based on necrotrophy in a fungal lineage spanning necrotrophs, biotrophs, endophytes, host generalists and specialists. PLOS ONE 7 (1), e29943.

Apostolakos P, P Livanos, TL Nikolakopoulou, B Galatis (2009). The role of callose in guardcell wall differentiation and stomatal pore formation in the fern Asplenium nidus. Annals of Botany 104 (7), 1373-1387.

Asada K (1992). Ascorbate peroxidase - a hydrogen peroxide-scavenging enzyme in plants. Physiologia plantarum 85, 235-241.

Ayaz FA, S Hayırlıoglu-Ayaz, S Alpay-Karaoglu, J Grúz, K Valentová, J Ulrichová et al. (2008). Phenolic acid contents of kale (Brassica oleraceae L. var. acephala DC.) extracts and their antioxidant and antibacterial activities. Food Chemistry 107 (1), 19-25.

Barbetti MJ, Li CX, Garg H, Li H, Banga SK, Banga SS et al (2011). Host resistance in oilseed Brassicas against Sclerotinia - renewed hope for managing a recalcitrant pathogen. 713-715, Proceedings of the $13^{\text {th }}$ International Rapeseed Congress, Prague, Czech Republic.

Bartlet E, G KIDDLE, I Williams, R Wallsgrove (1999). Wound-induced increases in the glucosinolate content of oilseed rape and their effect on subsequent herbivory by a crucifer specialist 91, 163-167.

Bartwal A, R Mall, P Lohani, SK Guru, S Arora (2012). Role of secondary metabolites and brassinosteroids in plant defense against environmental stresses. Journal of Plant Growth Regulation 32 (1), 216-232. 
Becker HC, GM Engqvist, B Karlsson (1995). Comparison of rapeseed cultivars and resynthesized lines based on allozyme and RFLP markers. Theoretical and Applied Genetics 91, 62-67.

Bednarek P, M Pislewka-Bednarek, A Svatos, B Schneider, et al. (2009). A glucosinolate metabolites pathway in living plant cells mediates broad-spectrum antifungal defense. Science 323, 101-106.

Beemster GTS, TI Baskin (1998). Analysis of cell division and elongation underlying the developmental acceleration of root growth in Arabidopsis thaliana. Plant Physiology 116 (4), 1515-1526.

Bell JG, J McEvoy, DR Tocher, F McGhee, PJ Campbell, JR Sargent (2001). Replacement of fish oil with rapeseed oil in diets of Atlantic salmon (Salmo salar) affects tissue lipid compositions and hepatocyte fatty acid metabolism. The Journal of Nutrition 131 (5), 1535-1543.

Benko-Iseppon A-M, P Winter, B Huettel, C Staginnus, FJ Muehlbauer, G Kahl (2003). Molecular markers closely linked to fusarium resistance genes in chickpea show significant alignments to pathogenesis-related genes located on Arabidopsis chromosomes 1 and 5. Theoretical and Applied Genetics 107 (2), 379-386.

Bennett R, RM Wallsgrove (1994). Secondary metabolites in plant defence mechanisms. New Physiologist 127, 617-633.

Bhadauria V, P Miraz, R Kennedy, S Banniza, Y Wei (2010). Dual trypan-aniline blue fluorescence staining methods for studying fungus-plant interactions. Biotechnic \& Histochemistry 85 (2), 99-105.

Boland GJ, R Hall (1994). Index of plant hosts of Sclerotinia sclerotiorum. Canadian Journal of Plant Pathology 16 (2), 93-108.

Bolton MD, BPHJ Thomma, BD Nelson (2006). Sclerotinia sclerotiorum (Lib.) de Bary: biology and molecular traits of a cosmopolitan pathogen. Molecular Plant Pathology 7 (1), 1-16.

Bradley CA, RA Henson, PM Porter, DG LeGare, LE del Río, SD Khot (2006a). Response of canola cultivars to Sclerotinia sclerotiorum in controlled and field environments. Plant Disease 90 (2), 215-219.

Bradley CA, HA Lamey, GJ Endres, RA Henson, BK Hanson, KR McKay et al. (2006b). Efficacy of fungicides for control of sclerotinia stem rot of canola. Plant Disease 90, 1129-1134.

Brandfass C, P Karlovsky (2008). Upscaled CTAB-based DNA extraction and real-time PCR assays for Fusarium culmorum and $F$. graminearum DNA in plant material with reduced sampling error. International Journal of Molecular Sciences 9 (11), 2306-2321.

brassica.info. URL: https://www.brassica.info/resource/plants/diversity_sets.php. Accessed $5^{\text {th }}$ October 2018.

Brown PD, JG Tokuhisa, M Reichelt, J Gershenzon (2003). Variation of glucosinolate accumulation among different organs and developmental stages of Arabidopsis thaliana. Phytochemistry 62 (3), 471-481. 
Bus A, N Körber, RJ Snowdon, B Stich (2011). Patterns of molecular variation in a specieswide germplasm set of Brassica napus. Theoretical and Applied Genetics 123 (8), 14131423.

Buxdorf K, H Yaffe, O Barda, M Levy (2013). The effects of glucosinolates and their breakdown products on necrotrophic fungi. PLOS ONE 8 (8), e70771.

Cessna SG, VE Sears, MB Dickman, PS Low (2000). Oxalic acid, a pathogenicity factor for Sclerotinia sclerotiorum, suppresses the oxidative burst of the host plant. The Plant Cell 12 (11), 2191-2200.

Chapple CCS, T Vogt, BE Ellis, CR Somerville (1992). An Arabidopsis mutant defective in the general phenylpropanoid pathway. The Plant Cell 4 (11), 1413-1424.

Chen S, BL Petersen, CE Olsen, A Schulz, BA Halkier (2001). Long-distance phloem transport of glucosinolates in Arabidopsis. Plant Physiology 127, 194-201.

Child RD, DE Evans, J Allen, GM Arnold (1993). Growth responses in oilseed rape (Brassica napus L.) to combined applications of the triazole chemicals triapenthenol and tebuconazole and interactions with gibberellin. Plant Growth Regulation 13 (2), 203212.

Christen O, Friedt W (eds) (2007). Winterraps. Das Handbuch für Profis. DLG-Verlag: Frankfurt am Main.

Clay NK, AM Adio, C Denoux, G Jander, FM Ausubel (2009). Glucosinolate metabolites required for an Arabidopsis innate immune response. Science 323 (5910), 95-101.

Clossais-Besnard N, F Larher (1991). Physiological role of glucosinolates in Brassica napus. Concentration and distribution pattern of glucosinolates among plant organs during a complete life cycle. Journal of the Science of Food and Agriculture 56 (1), 25-38.

Corwin JA, DJ Kliebenstein (2017). Quantitative resistance: more than just perception of a pathogen. The Plant Cell 29 (4), 655-665.

Crocoll C, BA Halkier, M Burow (2016). Analysis and quantification of glucosinolates. Current Protocols in Plant Biology 1, 385-409.

Davar R, R Darvishzadeh, A Majd, A Kharabian Masouleh, Y Ghosta (2012). The infection processes of Sclerotinia sclerotiorum in basal stem tissue of a susceptible genotype of Helianthus annuus L. Notulae Botanicae Horti Agrobotanici Cluj-Napoca 40 (1), 143-149.

Davidson AL, L Blahut-Beatty, A Itaya, Y Zhang, S Zheng, D Simmonds (2016). Histopathology of Sclerotinia sclerotiorum infection and oxalic acid function in susceptible and resistant soybean. Plant Pathology 65 (6), 878-887.

de Silva AP, MD Bolton, BD Nelson (2009). Transformation of Sclerotinia sclerotiorum with the green fluorescent protein gene and fluorescence of hyphae in four inoculated hosts. Plant Pathology 58 (3), 487-496.

del Río LE, CA Bradley, RA Henson, GJ Endres, BK Hanson, K McKay et al. (2007). Impact of sclerotinia stem rot on yield of canola. Plant Disease 91 (2), 191-194.

Derbyshire MC, M Denton-Giles (2016). The control of sclerotinia stem rot on oilseed rape (Brassica napus): current practices and future opportunities. Plant Pathology 65 (6), 859-877. 
Dickman MB, R Fluhr (2013). Centrality of host cell death in plant-microbe interactions. Annual Review of Phytopathology 51, 543-570.

Dicko MH, H Gruppen, C Barro, AS Traore, WJH van Berkel, AGJ Voragen (2005). Impact of phenolic compounds and related enzymes in sorghum varieties for resistance and susceptibility to biotic and abiotic stresses. Journal of Chemical Ecology 31 (11), 26712688.

Ding S-Y, Y-S Liu, Y Zeng, ME Himmel, JO Baker, EA Bayer (2012). How does plant cell wall nanoscale architecture correlate with enzymatic digestibility? Science 338 (6110), 10551060.

Ding Y, J Mei, Q Li, Y Liu, H Wan, L Wang et al. (2013). Improvement of Sclerotinia sclerotiorum resistance in Brassica napus by using B. oleracea. Genetic Resources and Crop Evolution 60 (5), 1615-1619.

Disi JO, J Mei, D Wei, Y Ding, W Qian (2014). Inheritance of leaf and stem resistance to Sclerotinia sclerotiorum in a cross between Brassica incana and Brassica oleracea var. alboglabra. The Journal of Agricultural Science 152 (01), 146-152.

Dixon RA, NL Paiva (1995). Stress-induced phenylpropanoid metabolism. The Plant Cell 7 (7), 1085-1097.

Dixon RA, L Achnine, P Kota, C-J Liu, MSS Reddy, L Wang (2002). The phenylpropanoid pathway and plant defence - a genomics perspective. Molecular Plant Pathology 3 (5), 371-390.

Dunker S (2005). Untersuchungen zur Schadwirkung von Sclerotinia sclerotiorum (Lib. de Bary) und Verticillium longisporum (comb. nov. Karapapa) in Winterraps (Brassica napus). Dissertation, Georg-August-University Göttingen.

Eynck C, B Koopmann, G Grunewaldt-Stoecker, P Karlovsky, A von Tiedemann (2007). Differential interactions of Verticillium longisporum and $V$. dahliae with Brassica napus detected with molecular and histological techniques. European Journal of Plant Pathology 118 (3), 259-274.

Eynck C, B Koopmann, P Karlovsky, A von Tiedemann (2009). Internal resistance in winter oilseed rape inhibits systemic spread of the vascular pathogen Verticillium longisporum. Phytopathology 99 (7), 802-811.

Eynck C, G Séguin-Swartz, WE Clarke, IAP Parkin (2012). Monolignol biosynthesis is associated with resistance to Sclerotinia sclerotiorum in Camelina sativa. Molecular Plant Pathology 13 (8), 887-899.

Fahey JW, AT Zalcmann, P Talalay (2001). The chemical diversity and distribution of glucosinolates and isothiocyanates among plants. Phytochemistry 56 (2), 5-51.

Fan ZX, WX Lei, XL Sun, B Yu, YZ Wang, GS Yang (2008). The association of Sclerotinia sclerotiorum resistance with glucosinolates in Brassica napus double-low DH population. Journal of Plant Pathology 90 (1), 43-48.

FAOSTAT (2018). URL: http://www.fao.org/faostat. Accessed $18^{\text {th }}$ April 2018.

Faulds CB, G Williamson (1999). The role of hydroxycinnamates in the plant cell wall. Journal of the Science of Food and Agriculture 79 (3), 393-395. 
Feng J, Y Long, L Shi, J Shi, G Barker, J Meng (2012). Characterization of metabolite quantitative trait loci and metabolic networks that control glucosinolate concentration in the seeds and leaves of Brassica napus. The New Phytologist 193 (1), 96-108.

Fieldsend J, GFJ Milford (1994). Changes in glucosinolates during crop development in single- and double-low genotypes of winter oilseed rape (Brassica napus): I. Production and distribution in vegetative tissues and developing pods during development and potential role in the recycling of sulphur within the crop. Annals of Applied Biology 124 (3), 531-542.

Foley RC, BN Kidd, JK Hane, JP Anderson, KB Singh (2016). Reactive oxygen species play a role in the infection of the necrotrophic fungi, Rhizoctonia solani in wheat. PLOS ONE 11 (3), e0152548.

Fraser CM, C Chapple (2011). The phenylpropanoid pathway in Arabidopsis. The arabidopsis book 9 , e0152.

French E, B-S Kim, AS Iyer-Pascuzzi (2016). Mechanisms of quantitative disease resistance in plants. Seminars in Cell \& Developmental Biology 56, 201-208.

Frerigmann H, M Piślewska-Bednarek, A Sánchez-Vallet, A Molina, E Glawischnig, T Gigolashvili et al. (2016). Regulation of pathogen-triggered tryptophan metabolism in Arabidopsis thaliana by MYB transcription factors and indole glucosinolate conversion products. Molecular Plant 9 (5), 682-695.

Garg H, K Sivasithamparam, SS Banga, MJ Barbetti (2008). Cotyledon assay as a rapid and reliable method of screening for resistance against Sclerotinia sclerotiorum in Brassica napus genotypes. Australasian Plant Pathology 37 (2), 106-111.

Garg H, C Atri, PS Sandhu, B Kaur, M Renton, SK Banga et al. (2010a). High level of resistance to Sclerotinia sclerotiorum in introgression lines derived from hybridization between wild crucifers and the crop Brassica species B. napus and B. juncea. Field Crops Research 117 (1), 51-58.

Garg H, H Li, K Sivasithamparam, J Kuo, MJ Barbetti (2010b). The infection processes of Sclerotinia sclerotiorum in cotyledon tissue of a resistant and a susceptible genotype of Brassica napus. Annals of Botany 106 (6), 897-908.

Garg H, H Li, K Sivasithamparam, MJ Barbetti (2013). Differentially expressed proteins and associated histological and disease progression changes in cotyledon tissue of a resistant and susceptible genotype of Brassica napus infected with Sclerotinia sclerotiorum. PLoS ONE 8 (6), e65205.

Gaspar T, C Kevers, C Penel, H Greppin, DM Reid, TA Thorpe (1996). Plant hormones and plant growth regulators in plant tissue culture. In Vitro Cellular and Developmental Biology - Plant 32, 272-289.

Ge XT, YP Li, ZJ Wan, MP You, PM Finnegan, SS Banga et al. (2012). Delineation of Sclerotinia sclerotiorum pathotypes using differential resistance responses on Brassica napus and B. juncea genotypes enables identification of resistance to prevailing pathotypes. Field Crops Research 127, 248-258.

Gerlach D (1984). Botanische Mikrotechnik - Eine Einführung, 3rd edn. Georg Thieme Verlag Stuttgart. 
Giamoustaris A, R Mithen (1997). Glucosinolates and disease resistance in oilseed rape (Brassica napus ssp. oleifera). Plant Pathology 46 (2), 271-275.

Glazebrook J (2005). Contrasting mechanisms of defense against biotrophic and necrotrophic pathogens. Annual Review of Phytopathology 43, 205-227.

Govrin EM, A Levine (2000). The hypersensitive response facilitates plant infection by the necrotrophic pathogen Botrytis cinerea. Current Biology 10 (13), 751-757.

Guimarães RL, HU Stotz (2004). Oxalate production by Sclerotinia sclerotiorum deregulates guard cells during infection. Plant Physiology 136 (3), 3703-3711.

Halkier BA, J Gershenzon (2006). Biology and biochemistry of glucosinolates. Annual Review of Plant Biology 57, 303-333.

Hao JJ, KV Subbarao, JM Duniway (2003). Germination of Sclerotinia minor and S. sclerotiorum sclerotia under various soil moisture and temperature combinations. Phytopathology 93 (4), 443-450.

Hegedus DD, SR Rimmer (2005). Sclerotinia sclerotiorum: when "to be or not to be" a pathogen? FEMS Microbiology Letters 251 (2), 177-184.

Herburger K, A Holzinger (2016). Aniline blue and calcofluor white staining of callose and cellulose in the streptophyte green algae Zygnema and Klebsormidium. Bio-protocol 6 (20), 1-5.

Höch K (2016). Phänotypisierung von Resistenzquellen und Charakterisierung von Resistenzfaktoren in Brassica-Arten gegenüber Sclerotinia sclerotiorum, dem Erreger der Weißstängeligkeit. Dissertation, Georg-August-University Göttingen.

Horbach R, AR Navarro-Quesada, W Knogge, HB Deising (2011). When and how to kill a plant cell: infection strategies of plant pathogenic fungi. Journal of Plant Physiology 168 (1), 51-62.

Hu X, DP Roberts, L Xie, JE Maul, C Yu, Y Li et al. (2014). Formulations of Bacillus subtilis BY2 suppress Sclerotinia sclerotiorum on oilseed rape in the field. Biological Control 70, 5464.

Huang HC, GC Kozub (1991). Temperature requirements for carpogenic germination of sclerotia of Sclerotinia sclerotiorum isolates of different geographic origin. Botanical Bulletin Academia Sinica 32, 279-286.

Huang HC, GC Kozub (1994). Germination of immature and mature sclerotia of Sclerotinia sclerotiorum. Botanical Bulletin Academia Sinica 35, 243-247.

Huang L, H Buchenauer, Q Han, X Zhang, Z Kang (2008). Ultrastructural and cytochemical studies on the infection process of Sclerotinia sclerotiorum in oilseed rape. Journal of Plant Diseases and Protection 115 (1), 9-16.

Iñiguez-Luy FL, ML Federico (2011). The genetics of Brassica napus L. In: Bancroft I \& Schmidt R (eds): Genetics and genomics of the Brassicaceae. Springer, New York Dordrecht Heidelberg London, 291-322.

Jamaux I, B Gelie, C Lamarque (1995). Early stages of infection of rapeseed petals and leaves by Sclerotinia sclerotiorum revealed by scanning electron microscopy. Plant Pathology 44 (1), 22-30. 
Johnson JM, I Sherameti, A Ludwig, PL Nongbri, C Sun, B Lou et al. (2011). Protocols for Arabidopsis thaliana and Piriformospora indica co-cultivation - A model system to study plant beneficial traits. Journal of Endocytobiosis and Cell Research, 101-113.

Jones JDG, JL Dangl (2006). The plant immune system. Nature 444 (7117), 323-329.

Joshi RK, S Megha, MH Rahman, U Basu, NNV Kav (2016). A global study of transcriptome dynamics in canola (Brassica napus L.) responsive to Sclerotinia sclerotiorum infection using RNA-Seq. Gene 590 (1), 57-67.

Kabbage M, B Williams, MB Dickman (2013). Cell death control: the interplay of apoptosis and autophagy in the pathogenicity of Sclerotinia sclerotiorum. PLoS Pathogens 9 (4), e1003287.

Kabbage M, O Yarden, MB Dickman (2015). Pathogenic attributes of Sclerotinia sclerotiorum: switching from a biotrophic to necrotrophic lifestyle. Plant Science 233, 53-60.

Khangura R, C Beard, A Hills (2018). Managing sclerotinia stem rot in canola. Department of Agriculture and Food. Australian Government.

[https://www.agric.wa.gov.au/canola/managing-sclerotinia-stem-rot-canola] Accessed 31 August 2018, 18:56

Kim KS, J-Y Min, MB Dickman (2008). Oxalic acid is an elicitor of plant programmed cell death during Sclerotinia sclerotiorum disease development. Molecular Plant-Microbe Interactions 21 (5), 605-612.

Kim H-J, C Chen, M Kabbage, MB Dickman (2011). Identification and characterization of Sclerotinia sclerotiorum NADPH oxidases. Applied and Environmental Microbiology 77 (21), 7721-7729.

Kimber D \& DI McGregor (1995). Brassica oilseeds: production \& utilisation. CAB International, UK.

Kirkegaard JA, MJ Robertson, P Hamblin, SJ Sprague (2006). Effect of blackleg and sclerotinia stem rot on canola yield in the high rainfall zone of southern New South Wales, Australia. Australian Journal of Agricultural Research 57 (2), 201.

Kliebenstein DJ, J Gershenzon, T Mitchell-Olds (2001). Comparative quantitative trait loci mapping for aliphatic, indolic and benzylic glucosinolate production in Arabidopsis thaliana leaves and seeds. Genetics 159, 359-370.

Koch S (2005). Entwicklung eines schlagspezifischen und schadensbezogenen Prognosemodells zur Bekämpfung von Sclerotinia sclerotiorum an Winterraps. Dissertation, Georg-August-University Göttingen.

Kull LS, TD Vuong, KS Powers, KM Eskridge, JR Steadman, GL Hartman (2003). Evaluation of resistance screening methods for sclerotinia stem rot of soybean and dry bean. Plant Disease 87 (12), 1471-1476.

Kumar M, L Campbell, S Turner (2016). Secondary cell walls: biosynthesis and manipulation. Journal of Experimental Botany 67 (2), 515-531.

Lamb C, RA Dixon (1997). The oxidative burst in plant disease resistance. Annual Review of Plant Physiology and Plant Molecular Biology 48, 251-275. 
Lattanzio V, VMT Lattanzio, A Cardinali (2006). Role of phenolics in the resistance mechanisms of plants against fungal pathogens and insects. Phytochemistry, 23-67.

Li Y, G Kiddle, R Bennett, K Doughty, R Wallsgrove (1999a). Variation in the glucosinolate content of vegetative tissues of Chinese lines of Brassica napus L. Annals of Applied Biology 134 (1), 131-136.

Li Y, G Kiddle, RN Bennett, RM Wallsgrove (1999b). Local and systemic changes in glucosinolates in Chinese and European cultivars of oilseed rape (Brassica napus L.) after inoculation with Sclerotinia sclerotiorum (stem rot). Annals of Applied Biology 134, 4558.

Li Y, J Chen, R Bennett, G Kiddle, R Wallsgrove, Y Huang, Y He (1999c). Breeding, inheritance, and biochemical studies on Brassica napus cv. Zhongyou 821: tolerance to Sclerotinia sclerotiorum (stem rot). Proceedings of the $10^{\text {th }}$ International Rapeseed Congress, Canberra, ACT.

Li YC, YD Li, DS Mei, YJ Huang, YS Xu, R Wallsgrove, G Kiddle, R Bennett (2001). Biochemical changes of Zhongyou 821 after inoculation with mycelia of Sclerotinia sclerotiorum. Chinese Journal of Oil Crop Sciences 23, 63-65.

Li R, R Rimmer, L Buchwaldt, AG Sharpe, G Séguin-Swartz, C Coutu et al. (2004). Interaction of Sclerotinia sclerotiorum with a resistant Brassica napus cultivar: expressed sequence tag analysis identifies genes associated with fungal pathogenesis. Fungal Genetics and Biology 41 (8), 735-753.

Li CX, H Li, AB Siddique, K Sivasithamparam, P Salisbury, SS Banga et al. (2007). The importance of the type and time of inoculation and assessment in the determination of resistance in Brassia napus and B. juncea to Sclerotinia sclerotiorum. Australian Journal of Agricultural Research 58 (12), 1198-1203.

Li J, Z Zhao, A Hayward, H Cheng, D Fu (2015). Integration analysis of quantitative trait loci for resistance to Sclerotinia sclerotiorum in Brassica napus. Euphytica 205 (2), 483-489.

Lima RB, VH Salvador, WD dos Santos, GA Bubna, A Finger-Teixeira, AR Soares et al. (2013). Enhanced lignin monomer production caused by cinnamic acid and its hydroxylated derivatives inhibits soybean root growth. PLOS ONE 8 (12), e80542.

Lin L, H Allemekinders, A Dansby, L Campbell, S Durance-Tod, A Berger et al. (2013). Evidence of health benefits of canola oil. Nutrition Reviews 71 (6), 370-385.

Liu Y (2004). Untersuchung der Variabilität von Sclerotinia sclerotiorum (Lib.) de Bary, dem Erreger der Weißstängeligkeit an Raps (Brassica napus L.) unter besonderer Berücksichtigung von Infektionsbiologie, Sortenreaktionen und gezielten Bekämpfungsmaßnahmen. Dissertation, Georg-August-University Göttingen.

Lumsden RD (1979). Histology and physiology in plant diseases caused by Sclerotinia species. Phytopathology 69 (8), 890-896.

Lumsden RD, RL Dow (1973). Histopathology of Sclerotinia sclerotiorum infection of bean. Phytopathology 63, 708-715.

Lumsden RD, WP Wergin (1980). Scanning-electron microscopy of incetion of bean by species of sclerotinia. Mycologia 72 (6), 1200-1209. 
Maher EA, NJ Bate, W Ni, Y Elkind, RA Dixon, CJ Lamb (1994). Increased disease susceptibility of transgenic tobacco plants with suppressed levels of performed phenylpropanoid products. Proceedings of the National Academy of Sciences 91, 78027806.

Malinovsky FG, JU Fangel, WGT Willats (2014). The role of the cell wall in plant immunity. Frontiers in Plant Science 5, 178.

Marciano P, P Di Lenna, P Magro (1983). Oxalic acid, cell wall-degrading enzymes and pH in pathogenesis and their significance in the virulence of two Sclerotinia sclerotiorum isolates on sunflower. Physiological Plant Pathology 22 (3), 339-345.

Mattila P, J Kumpulainen (2002). Determination of free and total phenolic acids in plantderived foods by HPLC with diode-array detection. Journal of Agricultural and Food Chemistry 50 (13), 3660-3667.

Mei J, L Qian, JO Disi, X Yang, Q Li, J Li et al. (2011). Identification of resistant sources against Sclerotinia sclerotiorum in Brassica species with emphasis on B. oleracea. Euphytica 177 (3), 393-399.

Mei J, Y Ding, K Lu, D Wei, Y Liu, JO Disi et al. (2013). Identification of genomic regions involved in resistance against Sclerotinia sclerotiorum from wild Brassica oleracea. Theoretical and Applied Genetics 126 (2), 549-556.

Miedes E, R Vanholme, W Boerjan, A Molina (2014). The role of the secondary cell wall in plant resistance to pathogens. Frontiers in Plant Science 5, 358.

Minorsky PV (2007). On the inside. Plant Physiology 145 (4), 1301-1302.

Mithen R (1992). Leaf glucosinolate profiles and their relationship to pest and disease resistance in oilseed rape. Euphytica 63, 71-83.

Mithen RF, BG Lewis, GR Fenwick (1986). In vitro activity of glucosinolates and their products against Leptosphaeria maculans. Transactions of the British Mycological Society 87 (3), 433-440.

Navabi ZK, IAP Parkin, JC Pires, Z Xiong, MR Thiagarajah, AG Good et al. (2010). Introgression of B-genome chromosomes in a doubled haploid population of Brassica napus x B. carinata. Genome 53 (8), 619-629.

Nicholson RL, R Hammerschmidt (1992). Phenolic compounds and their role in disease resistance. Annual Review of Phytopathology 30 (1), 369-389.

O'Brien JA, A Daudi, VS Butt, GP Bolwell (2012). Reactive oxygen species and their role in plant defence and cell wall metabolism. Planta 236 (3), 765-779.

Oliveira MB, RV de Andrade, MF Grossi-de-Sá, S Petrofeza (2015). Analysis of genes that are differentially expressed during the Sclerotinia sclerotiorum - Phaseolus vulgaris interaction. Frontiers in Microbiology 6, 1162.

Osbourn AE (1996). Preformed antimicrobial compounds and plant defense against fungal attack. The Plant Cell 8 (10), 1821-1831.

Passardi F, C Cosio, C Penel, C Dunand (2005). Peroxidases have more functions than a Swiss army knife. Plant cell reports 24 (5), 255-265. 
Peltier AJ, RD Hatfield, CR Grau (2009). Soybean stem lignin concentration relates to resistance to Sclerotinia sclerotiorum. Plant Disease 93 (2), 149-154.

Pfalz M, M Mukhaimar, F Perreau, J Kirk, CIC Hansen, CE Olsen et al. (2016). Methyl transfer in glucosinolate biosynthesis mediated by indole glucosinolate o-methyltransferase 5. Plant Physiology 172 (4), 2190-2203.

Poland JA, PJ Balint-Kurti, RJ Wisser, RC Pratt, RJ Nelson (2009). Shades of gray: the world of quantitative disease resistance. Trends in Plant Science 14 (1), 21-29.

Ponce de León I, M Montesano (2013). Activation of defense mechanisms against pathogens in mosses and flowering plants. International Journal of Molecular Sciences 14 (2), 3178-3200.

Prats E, ME Bazzalo, A León, JV Jorrín (2003). Accumulation of soluble phenolic compounds in sunflower capitula correlated with resistance to Sclerotinia sclerotiorum. Euphytica 132 (3), 321-329.

Pratt RG, SM Dabney, DA Mays (1988). New forage legume hosts of Sclerotinia trifoliorum and S. sclerotiorum in the southeastern United States. Plant Disease 72, 593-596.

Rademacher W (2000). Growth retardants: effects on gibberellin biosynthesis and other metabolic pathways. Annual Review of Plant Physiology and Plant Molecular Biology 51, 201-531.

Ratzinger A, N Riediger, A von Tiedemann, P Karlovsky (2009). Salicylic acid and salicylic acid glucoside in xylem sap of Brassica napus infected with Verticillium longisporum. Journal of Plant Research 122 (5), 571-579.

Reuveni R, M Shimoni, Z Karchi, J Kuc (1992). Peroxidase activity as a biochemical marker for resistance of muskmelon (Cucumis melo) to Pseudoperonospora cubensis. Phytopathology 82 (7), 749-753.

Ros Barceló A, LVG Ros, AE Carrasco (2007). Looking for syringyl peroxidases. Trends in Plant Science 12 (11), 486-491.

Rostás M, R Bennett, M Hilker (2002). Comparative physiological responses in chinese cabbage induced by herbyvory and fungal infection. Journal of Chemical Ecology 28 (12), 2449-2463.

Sachs RM, CF Bretz, A Lang (1959). Shoot histogenesis: the early effects of gibberellin upon stem elongation in two rosette plants. American Journal of Botany 46 (5), 376-384.

Sanchez-Vallet A, B Ramos, P Bednarek, G López, M Piślewska-Bednarek, P Schulze-Lefert et al. (2010). Tryptophan-derived secondary metabolites in Arabidopsis thaliana confer non-host resistance to necrotrophic Plectosphaerella cucumerina fungi. The Plant Journal 63 (1), 115-127.

Sattler SE, DL Funnell-Harris (2013). Modifying lignin to improve bioenergy feedstocks: strengthening the barrier against pathogens? Frontiers in Plant Science 4, 1-8.

Schenk ST, A Schikora (2015). Staining of callose depositions in root and leaf tissues. Bioprotocol 5 (6), 1-4. 
Schlaeppi K, E Abou-Mansour, A Buchala, F Mauch (2010). Disease resistance of Arabidopsis to Phytophthora brassicae is established by the sequential action of indole glucosinolates and camalexin. The Plant Journal 62 (5), 840-851.

Seifbarghi S, MH Borhan, Y Wei, C Coutu, SJ Robinson, DD Hegedus (2017). Changes in the Sclerotinia sclerotiorum transcriptome during infection of Brassica napus. BMC Genomics 18 (1), 266.

Sønderby IE, F Geu-Flores, BA Halkier (2010). Biosynthesis of glucosinolates - gene discovery and beyond. Trends in Plant Science 15 (5), 283-290.

Sotelo T, P Soengas, P Velasco, VM Rodríguez, ME Cartea (2014). Identification of metabolic QTLs and candidate genes for glucosinolate synthesis in Brassica oleracea leaves, seeds and flower buds. PLOS ONE 9 (3), e91428.

St Clair DA (2010). Quantitative disease resistance and quantitative resistance loci in breeding. Annual Review of Phytopathology 48, 247-268.

Stotz HU, Y Sawada, Y Shimada, MY Hirai, E Sasaki, M Krischke et al. (2011). Role of camalexin, indole glucosinolates, and side chain modification of glucosinolate-derived isothiocyanates in defense of Arabidopsis against Sclerotinia sclerotiorum. The Plant Journal 67 (1), 81-93.

Tariq VN, P Jeffries (1984). Appressorium formation by Sclerotinia sclerotiorum: Scanning electron microscopy. Transactions of the British Mycological Society 82 (4), 645-651.

Tariq VN, P Jeffries (1986). Ultrastructure of penetration of Phaseolus spp. by Sclerotinia sclerotiorum. Canadian Journal of Botany 64, 2909-2915.

Taylor A, E Coventry, JE Jones, JP Clarkson (2015). Resistance to a highly aggressive isolate of Sclerotinia sclerotiorum in a Brassica napus diversity set. Plant Pathology 64 (4), 932940.

Taylor A, K Rana, C Handy, JP Clarkson (2018). Resistance to Sclerotinia sclerotiorum in wild Brassica species and the importance of Sclerotinia subarctica as a Brassica pathogen. Plant Pathology 67 (2), 433-444.

Troian RF, AS Steindorff, MH Ramada, W Arruda, CJ Ulhoa (2014). Mycoparasitism studies of Trichoderma harzianum against Sclerotinia sclerotiorum: evaluation of antagonism and expression of cell wall-degrading enzymes genes. Biotechnology Letters 36 (10), 2095-2101.

Uloth MB, MP You, PM Finnegan, SS Banga, SK Banga, PS Sandhu et al. (2013). New sources of resistance to Sclerotinia sclerotiorum for crucifer crops. Field Crops Research 154, 4052.

Uloth M, MP You, PM Finnegan, SS Banga, H Yi, MJ Barbetti (2014). Seedling resistance to Sclerotinia sclerotiorum as expressed across diverse cruciferous species. Plant Disease 98 (2), 184-190.

Uloth MB, PL Clode, MP You, MJ Barbetti (2016). Attack modes and defence reactions in pathosystems involving Sclerotinia sclerotiorum, Brassica carinata, B. juncea and $B$. napus. Annals of Botany 117 (1), 79-95. 
van Kan JAL (2006). Licensed to kill: the lifestyle of a necrotrophic plant pathogen. Trends in Plant Science 11 (5), 247-253.

Vogt T (2010). Phenylpropanoid biosynthesis. Molecular Plant 3 (1), 2-20.

Voigt CA (2014). Callose-mediated resistance to pathogenic intruders in plant defenserelated papillae. Frontiers in Plant Science 5, 168.

von Tiedemann A (1997). Evidence for a primary role of active oxygen species in induction of host cell death during infection of bean leaves with Botrytis cinerea. Physiological and Molecular Plant Pathology 50 (3), 151-166.

Wang H-Z, G-H Liu, Y-B Zheng, X-F Wang, Q Yang (2004). Breeding of a Brassica napus cultivar Zhongshuang No. 9 with high-resistance to Sclerotinia sclerotiorum and dynamics of its important defense enzymae activity. Scientia Agricultura Sinica 37 (1), 23-28.

Wei D, J Mei, Y Fu, JO Disi, J Li, W Qian (2014). Quantitative trait loci analyses for resistance to Sclerotinia sclerotiorum and flowering time in Brassica napus. Molecular Breeding 34 (4), 1797-1804.

Wei L, H Jian, K Lu, F Filardo, N Yin, L Liu et al. (2015). Genome-wide association analysis and differential expression analysis of resistance to sclerotinia stem rot in Brassica napus. Plant Biotechnology Journal, 1-13.

Wilcox HE, LC Marsh (2009). Staining plant tissues with Chlorazol Black E and Pianese III-B. Stain Technology 39 (2), 81-86.

Williams B, M Kabbage, H-J Kim, R Britt, MB Dickman (2011). Tipping the balance: Sclerotinia sclerotiorum secreted oxalic acid suppresses host defenses by manipulating the host redox environment. PLoS Pathogens 7 (6), e1002107.

Wu BM, KV Subbarao (2008). Effects of soil temperature, moisture, and burial depths on carpogenic germination of Sclerotinia sclerotiorum and S. minor. Phytopathology 98 (10), 1144-1152.

Wu J, G Cai, J Tu, L Li, S Liu, X Luo et al. (2013). Identification of QTLs for resistance to sclerotinia stem rot and BnaC.IGMT5.a as a candidate gene of the major resistant QTL SRC6 in Brassica napus. PLoS ONE 8 (7), e67740.

Xu L, M Xiang, D White, W Chen (2015). pH dependency of sclerotial development and pathogenicity revealed by using genetically defined oxalate-minus mutants of Sclerotinia sclerotiorum. Environmental Microbiology 17 (8), 2896-2909.

Wulf, T (2011). Phänotypisierung von Raps- und anderen Brassica-Genotypen zur Identifizierung von Resistenzquellen gegenüber Sclerotinia sclerotiorum. Dissertation, Georg-August-University Göttingen.

Yadeta KA, BPHJ Thomma (2013). The xylem as battleground for plant hosts and vascular wilt pathogens. Frontiers in Plant Science 4, 97.

Yeats TH, JKC Rose (2013). The formation and function of plant cuticles. Plant Physiology 163 (1), 5-20.

Yin Y, L Ding, X Liu, J Yang, Z Ma (2009). Detection of Sclerotinia sclerotiorum in planta by a real-time PCR assay. Journal of Phytopathology 157 (7-8), 465-469. 
Yin X, B Yi, W Chen, W Zhang, J Tu, WGD Fernando et al. (2010). Mapping of QTLs detected in a Brassica napus DH population for resistance to Sclerotinia sclerotiorum in multiple environments. Euphytica 173 (1), 25-35.

You MP, MB Uloth, XX Li, SS Banga, SK Banga, MJ Barbetti (2016). Valuable new resistances ensure improved management of sclerotinia stem rot (Sclerotinia sclerotiorum) in horticultural and oilseed brassica species. Journal of Phytopathology 164 (5), 291-299.

Young CS, CP Werner (2012). Infection routes for Sclerotinia sclerotiorum in apetalous and fully petalled winter oilseed rape. Plant Pathology 61 (4), 730-738.

Zamani-Noor N, J Knüfer (2018). Effects of host plant resistance and fungicide application on phoma stem canker, growth parameters and yield of winter oilseed rape. Crop Protection 112, 313-321.

Zhao J, J Meng (2003a). Genetic analysis of loci associated with partial resistance to Sclerotinia sclerotiorum in rapeseed (Brassica napus L.). Theoretical and Applied Genetics 106 (4), 759-764.

Zhao J, J Meng (2003b). Detection of loci controlling seed glucosinolate content and their association with Sclerotinia resistance in Brassica napus. Plant Breeding 122 (1), 19-23.

Zhao J, AJ Peltier, J Meng, TC Osborn, CR Grau (2004). Evaluation of sclerotinia stem rot resistance in oilseed Brassica napus using a petiole inoculation technique under greenhouse conditions. Plant Disease 88 (9), 1033-1039.

Zhao J, LC Davis, R Verpoorte (2005). Elicitor signal transduction leading to production of plant secondary metabolites. Biotechnology Advances 23 (4), 283-333.

Zhao J, JA Udall, PA Quijada, CR Grau, J Meng, TC Osborn (2006). Quantitative trait loci for resistance to Sclerotinia sclerotiorum and its association with a homeologous nonreciprocal transposition in Brassica napus L. Theoretical and Applied Genetics 112 (3), 509-516.

Zhao J, J Wang, L An, RW Doerge, ZJ Chen, CR Grau et al. (2007). Analysis of gene expression profiles in response to Sclerotinia sclerotiorum in Brassica napus. Planta 227 (1), 13-24.

Zhao J, L Buchwaldt, SR Rimmer, A Sharpe, L McGregor, D Bekkaoui et al. (2009). Patterns of differential gene expression in Brassica napus cultivars infected with Sclerotinia sclerotiorum. Molecular Plant Pathology 10 (5), 635-649.

Zhao S, PA Tuan, X Li, YB Kim, H Kim, CG Park et al. (2013). Identification of phenylpropanoid biosynthetic genes and phenylpropanoid accumulation by transcriptome analysis of Lycium chinense. BMC Genomics 14, 802.

Zhao Q, RA Dixon (2014). Altering the cell wall and its impact on plant disease: from forage to bioenergy. Annual Review of Phytopathology 52, 69-91.

Zhao X, Y Han, Y Li, D Liu, M Sun, Y Zhao et al. (2015). Loci and candidate gene identification for resistance to Sclerotinia sclerotiorum in soybean (Glycine max L. Merr.) via association and linkage maps. The Plant Journal 82 (2), 245-255.

Zheng $X$ (2018). Verticillium longisporum on oilseed rape (Brassica napus L.) - Differential roles of salicylic acid, seed transmission and plant colonization. Dissertation, GeorgAugust-University Göttingen. 


\section{Annex}

Tab. 1A Brassica napus Diversity Fixed Foundation Set (BnDFFS) completed with own genotypes for greenhouse resistance screening against S. sclerotiorum. Number, name and growth type of genotypes are shown as well as if plants were flowering ( $y$; marked dark gray), partly flowering (ok; marked light gray) or not flowering with a tough stem (n) when inoculated.

\begin{tabular}{|c|c|c|c|c|}
\hline Number & Name & Growth type & Flower & Comment \\
\hline BnASSYST-513 & Chuanyou 2 & Chinese & $y$ & \\
\hline BnASSYST-520 & Ningyou 7 & Chinese & $y$ & \\
\hline BnASSYST-229 & SWU Chinese 1 & Chinese & y & \\
\hline BnASSYST-230 & SWU Chinese 2 & Chinese & y & \\
\hline BnASSYST-231 & SWU Chinese 3 & Chinese & $y$ & \\
\hline BnASSYST-232 & SWU Chinese 5 & Chinese & $y$ & \\
\hline BnASSYST-235 & SWU Chinese 8 & Chinese & $y$ & \\
\hline BnASSYST-236 & sWU Chinese 9 & Chinese & $y$ & \\
\hline BnASSYST-529 & Xiangyou 15 & Chinese & $y$ & \\
\hline BnASSYST-530 & Zhongshuang II & Chinese & $y$ & \\
\hline BnASSYST-237 & Zhouyou & Chinese & $y$ & \\
\hline BnASSYST-224 & WILD ACCESSION & Exotics semiwinter & $y$ & 1 \\
\hline BnASSYST-217 & EVVIN & exotics var. napus & $y$ & \\
\hline BnASSYST-208 & COUVE NABICA & Leafy vegetable & $y$ & \\
\hline BnASSYST-206 & Brauner Schnittkohl & Siberian Kale & $y$ & \\
\hline BnASSYST-242 & BRUTOR & Spring OSR & $y$ & \\
\hline BnASSYST-274 & Ceska Krajova & Spring OSR & $y$ & \\
\hline BnASSYST-323 & Daichousen (mizuyasu) & Spring OSR & y & \\
\hline BnASSYST-238 & Drakkar & Spring OSR & $y$ & \\
\hline BnASSYST-325 & Erake & Spring OSR & $y$ & \\
\hline BnASSYST-326 & Furax & Spring OSR & $y$ & \\
\hline BnASSYST-251 & KARAT & Spring OSR & $y$ & \\
\hline BnASSYST-256 & Karoo-057DH & Spring OSR & $y$ & \\
\hline BnASSYST-349 & Loras & Spring OSR & $y$ & \\
\hline BnASSYST-300 & Magma & Spring OSR & y & \\
\hline BnASSYST-268 & MAZOWIECKI & Spring OSR & $y$ & \\
\hline BnASSYST-373 & Rucabo & Spring OSR & $y$ & \\
\hline BnASSYST-239 & STELLAR DH & Spring OSR & $y$ & \\
\hline BnASSYST-307 & Tribune & Spring OSR & $y$ & \\
\hline BnASSYST-284 & Tribute & Spring OSR & $y$ & \\
\hline BnASSYST-270 & WEIHENSTEPHANER & Spring OSR & $y$ & \\
\hline BnASSYST-240 & WESTAR DH & Spring OSR & $y$ & \\
\hline BnASSYST-399 & Zairai Chousenshu & Spring OSR & $y$ & \\
\hline BnASSYST-417 & Aberdeenshire Prize & Swede & $y$ & 4 \\
\hline BnASSYST-418 & Altasweet & Swede & $y$ & \\
\hline BnASSYST-426 & Drummonds Purple Top & Swede & $y$ & 4 \\
\hline BnASSYST-410 & Hueguenot & Swede & $y$ & \\
\hline BnASSYST-414 & SENSATION NZ & Swede & $y$ & 1 \\
\hline
\end{tabular}




\begin{tabular}{|c|c|c|c|}
\hline BnASSYST-436 & Tina & Swede & $\mathrm{y}$ \\
\hline BnASSYST-401 & VIGE DH1 & Swede & y \\
\hline BnASSYST-438 & YORK & Swede & $y$ \\
\hline BnASSYST-204 & Q100 & Synthetic & y \\
\hline BnASSYST-191 & Binera & Winter fodder & $\mathrm{y}$ \\
\hline BnASSYST-073 & Amor & Winter OSR & y \\
\hline BnASSYST-040 & Apex & Winter OSR & $y$ \\
\hline BnASSYST-090 & APEX-93xGINYOU_3 & Winter OSR & y \\
\hline BnASSYST-072 & Beluga & Winter OSR & $\mathrm{y}$ \\
\hline BnASSYST-016 & Boston & Winter OSR & y \\
\hline BnASSYST-510 & Cabriolet & Winter OSR & $y$ \\
\hline BnASSYST-076 & Caramba & Winter OSR & y \\
\hline BnASSYST-511 & Castille & Winter OSR & y \\
\hline BnASSYST-512 & Catana & Winter OSR & $\mathrm{y}$ \\
\hline BnASSYST-037 & Contact & Winter OSR & y \\
\hline \multirow[t]{2}{*}{ BnASSYST-055 } & Expert & Winter OSR & $\mathrm{y}$ \\
\hline & Express & Winter OSR & $\mathrm{y}$ \\
\hline BnASSYST-045 & Fortis & Winter OSR & $\mathrm{y}$ \\
\hline BnASSYST-096 & HANSEN x GASPARD DH LINE & Winter OSR & $\mathrm{y}$ \\
\hline BnASSYST-517 & Huron x Navajo & Winter OSR & ok \\
\hline BnASSYST-029 & Idol & Winter OSR & $\mathrm{y}$ \\
\hline BnASSYST-121 & JetNeuf & Winter OSR & $y$ \\
\hline BnASSYST-024 & Ladoga & Winter OSR & y \\
\hline BnASSYST-128 & Leopard & Winter OSR & $\mathrm{y}$ \\
\hline BnASSYST-102 & Lesira & Winter OSR & $\mathrm{y}$ \\
\hline BnASSYST-035 & Lipton & Winter OSR & $y$ \\
\hline BnASSYST-007 & Milena & Winter OSR & y \\
\hline BnASSYST-124 & Mohican & Winter OSR & $y$ \\
\hline BnASSYST-039 & OASE & Winter OSR & y \\
\hline BnASSYST-110 & Olimpiade & Winter OSR & y \\
\hline BnASSYST-020 & Pacific & Winter OSR & $\mathrm{y}$ \\
\hline BnASSYST-521 & Palmedor & Winter OSR & $\mathrm{y}$ \\
\hline BnASSYST-011 & Picasso & Winter OSR & $\mathrm{y}$ \\
\hline BnASSYST-005 & Pirola & Winter OSR & y \\
\hline BnASSYST-081 & Pollen & Winter OSR & $\mathrm{y}$ \\
\hline BnASSYST-082 & Prince & Winter OSR & y \\
\hline BnASSYST-098 & RAFAL DH1 & Winter OSR & $\mathrm{y}$ \\
\hline BnASSYST-015 & Rapid & Winter OSR & y \\
\hline BnASSYST-524 & Rocket & Winter OSR & $\mathrm{y}$ \\
\hline BnASSYST-051 & Sansibar & Winter OSR & y \\
\hline BnASSYST-021 & Savannah & Winter OSR & y \\
\hline BnASSYST-106 & SHANNON $x$ WINNER DH LINE & Winter OSR & y \\
\hline BnASSYST-069 & SLM 0413 & Winter OSR & y \\
\hline BnASSYST-070 & SLM 0512 & Winter OSR & $\mathrm{y}$ \\
\hline BnASSYST-203 & Taisetsu & Winter OSR & $\mathrm{y}$ \\
\hline BnASSYST-099 & TAPIDOR DH & Winter OSR & y \\
\hline BnASSYST-527 & Temple & Winter OSR & y \\
\hline
\end{tabular}




\begin{tabular}{|c|c|c|c|}
\hline BnASSYST-179 & Wolynski & Winter OSR & y \\
\hline \multirow[t]{2}{*}{ BnASSYST-526 } & Shengliyoucai & Chinese & ok \\
\hline & Zhongyou 821 & Chinese & $y$ \\
\hline BnASSYST-282 & SvalšfÕs Gulle & Spring OSR & $y$ \\
\hline BnASSYST-290 & Ability & Spring OSR & $\mathrm{n}$ \\
\hline BnASSYST-272 & Alku & Spring OSR & $\mathrm{n}$ \\
\hline BnASSYST-275 & Duplo & Spring OSR & ok \\
\hline BnASSYST-258 & N01D-1330 & Spring OSR & $\mathrm{y}$ \\
\hline BnASSYST-259 & N02D-1952 & Spring OSR & $\mathrm{y}$ \\
\hline BnASSYST-367 & Pobeda & Spring OSR & ok \\
\hline \multirow[t]{2}{*}{ BnASSYST-283 } & Topas & Spring OSR & $\mathrm{y}$ \\
\hline & Loras & Spring OSR & ok \\
\hline BnASSYST-421 & Bangholm PT & Swede & $\mathrm{n}$ \\
\hline BnASSYST-403 & Fortin Family & Swede & $\mathrm{n}$ \\
\hline BnASSYST-448 & Wilhelmsburger & Swede & $\mathrm{n}$ \\
\hline BnASSYST-133 & Baltia & Winter OSR & $\mathrm{n}$ \\
\hline BnASSYST-091 & Bienvenu DH4 & Winter OSR & $\mathrm{n}$ \\
\hline BnASSYST-093 & CANBERRA x COURAGE DH LINE & Winter OSR & ok \\
\hline \multirow[t]{2}{*}{ BnASSYST-123 } & Falcon & Winter OSR & $\mathrm{n}$ \\
\hline & Falcon-UGO & Winter OSR & y \\
\hline BnASSYST-107 & Janetzkis Schlesischer & Winter OSR & $\mathrm{y}$ \\
\hline BnASSYST-097 & MADRIGAL x RECITAL DH LINE & Winter OSR & ok \\
\hline BnASSYST-002 & Remy & Winter OSR & ok \\
\hline BnASSYST-176 & Trebicska & Winter OSR & ok \\
\hline BnASSYST-030 & Vivol & Winter OSR & ok \\
\hline BnASSYST-218 & GROENE GRONINGER SNIJMOES & Siberian Kale & $\mathrm{n}$ \\
\hline BnASSYST-271 & Liho & Spring fodder & $x$ \\
\hline BnASSYST-273 & Bronowski & Spring OSR & $\mathrm{n}$ \\
\hline BnASSYST-245 & CRESOR & Spring OSR & $\mathrm{n}$ \\
\hline BnASSYST-261 & CUBS ROOT & Spring OSR & $\mathrm{n}$ \\
\hline BnASSYST-263 & ERGLU & Spring OSR & $\mathrm{n}$ \\
\hline BnASSYST-264 & HELIOS & Spring OSR & $\mathrm{n}$ \\
\hline BnASSYST-359 & Odin & Spring OSR & $\mathrm{n}$ \\
\hline BnASSYST-361 & Omega & Spring OSR & $\mathrm{n}$ \\
\hline BnASSYST-221 & RAPID CYCLING RAPE (CrGC5) & Spring OSR & $\mathrm{n}$ \\
\hline BnASSYST-260 & SURPASS400-024DH & Spring OSR & $\mathrm{n}$ \\
\hline BnASSYST-269 & TANTAL & Spring OSR & $\mathrm{n}$ \\
\hline BnASSYST-394 & Willi & Spring OSR & $\mathrm{n}$ \\
\hline BnASSYST-241 & YUDAL & Spring OSR & $\mathrm{n}$ \\
\hline BnASSYST-411 & Jaune a collet vert & Swede & $\mathrm{n}$ \\
\hline BnASSYST-122 & Cobra & Winter OSR & $\mathrm{n}$ \\
\hline BnASSYST-213 & ABUKUMA NATANE & Winter OSR & $\mathrm{n}$ \\
\hline BnASSYST-009 & Agalon & Winter OSR & $\mathrm{n}$ \\
\hline BnASSYST-001 & Alesi & Winter OSR & $\mathrm{n}$ \\
\hline BnASSYST-089 & Amber $x$ Commanche & Winter OSR & $\mathrm{n}$ \\
\hline BnASSYST-061 & Aragon & Winter OSR & $\mathrm{n}$ \\
\hline BnASSYST-509 & Cabernet & Winter OSR & $\mathrm{n}$ \\
\hline
\end{tabular}




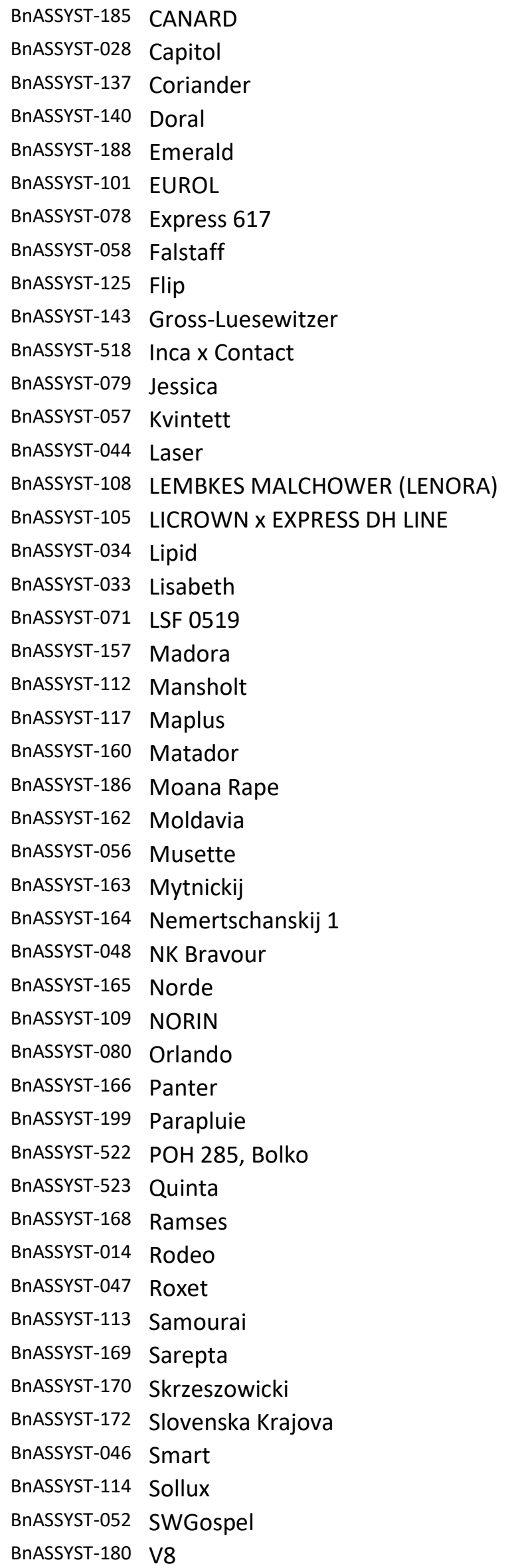




\begin{tabular}{|c|c|c|c|c|}
\hline BnASSYST-053 & Verona & Winter OSR & $n$ & \\
\hline BnASSYST-060 & Viking & Winter OSR & $\mathrm{n}$ & \\
\hline BnASSYST-528 & Vision & Winter OSR & $\mathrm{n}$ & \\
\hline BnASSYST-068 & Zephir & Winter OSR & $n$ & \\
\hline BnASSYST-257 & Monty-028DH & Spring OSR & $x$ & - \\
\hline BnASSYST-115 & Akela & Winter OSR & $x$ & $b$ \\
\hline BnASSYST-118 & Askari & Winter OSR & $x$ & $\mathrm{~b}$ \\
\hline BnASSYST-119 & Lirabon & Winter OSR & $x$ & $b$ \\
\hline BnASSYST-126 & Zenith & Winter OSR & $x$ & $\mathrm{~b}$ \\
\hline BnASSYST-127 & Phil & Winter OSR & $x$ & $b$ \\
\hline BnASSYST-129 & RESYN-H048 & Winter OSR & $x$ & $b$ \\
\hline BnASSYST-138 & Diamant & Winter OSR & $x$ & $b$ \\
\hline BnASSYST-139 & Dippes & Winter OSR & $x$ & $b$ \\
\hline BnASSYST-149 & Krapphauser & Winter OSR & $x$ & $b$ \\
\hline BnASSYST-150 & Kromerska & Winter OSR & $x$ & $\mathrm{~b}$ \\
\hline BnASSYST-173 & Sobotowsky & Winter OSR & $x$ & $b$ \\
\hline BnASSYST-178 & Vinnickij 15/59 & Winter OSR & $x$ & $b$ \\
\hline BnASSYST-212 & Slapska,Slapy & Winter OSR & $x$ & $b$ \\
\hline BnASSYST-514 & Dimension & Winter OSR & $x$ & $b$ \\
\hline BnASSYST-209 & Ragged Jack & Rape kale & $x$ & $b$ \\
\hline BnASSYST-211 & Siberische Boerenkohl & Siberian Kale & $x$ & $b$ \\
\hline BnASSYST-271 & Liho & Spring fodder & $x$ & not germinated \\
\hline BnASSYST-418 & Altasweet & Swede & $x$ & $\mathrm{~b}$ \\
\hline
\end{tabular}

$y-60 \%$ of the plant were flowering when inoculated, $n$ - plants had elongated but tough stem and did not flower when inoculated, ok - less than $50 \%$ of the plant were flowering when inoculated; 1 - thin stem, very early flowering, 2 - small, thin plants, 3 - beet root, 4 - late flowering, $b$ - expelled when plants did not reach flowering stage 
Tab. 2A Mean lesion length of accessions 7 days post inoculated with S. sclerotiorum being different from either the susceptible or the partially resistant reference line Loras or Zhongyou 821. Letters indicate significant differences (Kruskal-Wallis ANOVA, $P<0.05$ ).

\begin{tabular}{|c|c|c|c|c|}
\hline \multirow{2}{*}{$\begin{array}{l}\begin{array}{l}\text { Accession } \\
\text { number }\end{array} \\
\text { BnASSYST-361 }\end{array}$} & \multirow{2}{*}{$\begin{array}{l}\text { Variety } \\
\text { Omega }\end{array}$} & \multicolumn{3}{|c|}{ Mean lesion length $[\mathrm{mm}] \pm \mathrm{SD}$} \\
\hline & & $2.2 \pm$ & 1.0 & c \\
\hline BnASSYST-522 & POH 285, Bolko & $3.6 \pm$ & 1.0 & c \\
\hline BnASSYST-140 & Doral & $3.8 \pm$ & 0.5 & c \\
\hline BnASSYST-268 & MAZOWIECKI & $4.0 \pm$ & 1.0 & c \\
\hline BnASSYST-033 & Lisabeth & $4.0 \pm$ & 1.5 & c \\
\hline BnASSYST-274 & Ceska Krajova & $4.2 \pm$ & 0.9 & c \\
\hline BnASSYST-261 & CUBS ROOT & $4.4 \pm$ & 2.8 & c \\
\hline BnASSYST-241 & YUDAL & $4.6 \pm$ & 0.6 & c \\
\hline BnASSYST-221 & RAPID CYCLING RAPE (CrGC5) & $4.9 \pm$ & 2.7 & c \\
\hline BnASSYST-060 & Viking & $5.4 \pm$ & 3.2 & c \\
\hline BnASSYST-114 & Sollux & $5.4 \pm$ & 1.5 & c \\
\hline BnASSYST-080 & Orlando & $5.6 \pm$ & 2.2 & c \\
\hline BnASSYST-001 & Alesi & $5.7 \pm$ & 2.5 & c \\
\hline BnASSYST-326 & Furax & $6.2 \pm$ & 5.6 & c \\
\hline BnASSYST-166 & Panter & $6.3 \pm$ & 3.9 & c \\
\hline BnASSYST-113 & Samourai & $6.5 \pm$ & 1.5 & c \\
\hline BnASSYST-168 & Ramses & $6.6 \pm$ & 1.3 & c \\
\hline BnASSYST-034 & Lipid & $6.6 \pm$ & 2.6 & c \\
\hline BnASSYST-046 & Smart & $7.2 \pm$ & 5.3 & c \\
\hline BnASSYST-117 & Maplus & $7.4 \pm$ & 5.5 & c \\
\hline BnASSYST-014 & Rodeo & $7.6 \pm$ & 7.6 & c \\
\hline BnASSYST-009 & Agalon & $7.7 \pm$ & 5.8 & c \\
\hline BnASSYST-105 & LICROWN x EXPRESS DH LINE & $8.0 \pm$ & 9.7 & c \\
\hline BnASSYST-394 & Willi & $8.2 \pm$ & 4.9 & c \\
\hline BnASSYST-260 & SURPASS400-024DH & $8.3 \pm$ & 4.8 & c \\
\hline BnASSYST-125 & Flip & $8.3 \pm$ & 15.4 & c \\
\hline BnASSYST-101 & EUROL & $9.1 \pm$ & 8.1 & c \\
\hline BnASSYST-058 & Falstaff & $9.3 \pm$ & 8.2 & c \\
\hline BnASSYST-109 & NORIN & $9.8 \pm$ & 4.7 & c \\
\hline BnASSYST-170 & Skrzeszowicki & $9.8 \pm$ & 5.1 & c \\
\hline BnASSYST-128 & Leopard & $9.9 \pm$ & 5.1 & c \\
\hline BnASSYST-047 & Roxet & $10.0 \pm$ & 8.1 & c \\
\hline BnASSYST-048 & NK Bravour & $10.2 \pm$ & 8.1 & c \\
\hline BnASSYST-165 & Norde & $10.4 \pm$ & 3.6 & c \\
\hline BnASSYST-112 & Mansholt & $11.2 \pm$ & 8.2 & c \\
\hline BnASSYST-078 & Express 617 & $12.1 \pm$ & 18.0 & c \\
\hline BnASSYST-056 & Musette & $12.3 \pm$ & 5.2 & c \\
\hline BnASSYST-421 & Bangholm PT & $12.4 \pm$ & 14.8 & c \\
\hline BnASSYST-143 & Gross-Luesewitzer & $12.4 \pm$ & 10.1 & c \\
\hline BnASSYST-079 & Jessica & $12.8 \pm$ & 9.0 & c \\
\hline BnASSYST-528 & Vision & $13.1 \pm$ & 11.0 & c \\
\hline BnASSYST-411 & Jaune a collet vert & $13.3 \pm$ & 15.9 & c \\
\hline
\end{tabular}




\begin{tabular}{|c|c|c|c|c|c|c|}
\hline BnASSYST-068 & Zephir & 13.9 & & 12.0 & & c \\
\hline BnASSYST-108 & LEMBKES MALCHOWER (LENORA) & 14.7 & \pm & 16.5 & & c \\
\hline BnASSYST-089 & Amber $\times$ Commanche & 15.8 & \pm & 17.3 & & c \\
\hline BnASSYST-172 & Slovenska Krajova & 16.2 & \pm & 6.4 & & c \\
\hline BnASSYST-029 & Idol & 17.2 & \pm & 17.3 & & c \\
\hline BnASSYST-107 & Janetzkis Schlesischer & 17.3 & \pm & 20.0 & & C \\
\hline BnASSYST-133 & Baltia & 18.0 & \pm & 13.1 & & c \\
\hline BnASSYST-162 & Moldavia & 18.3 & \pm & 16.6 & & $b$ \\
\hline BnASSYST-096 & HANSEN x GASPARD DH LINE & 23.0 & \pm & 27.7 & & c \\
\hline \multirow[t]{3}{*}{ BnASSYST-093 } & CANBERRA x COURAGE DH LINE & 31.3 & \pm & 45.2 & & c \\
\hline & Zhongyou 821 & 34.1 & \pm & 25.8 & & b c \\
\hline & Loras & 76.2 & \pm & 24.1 & $a b$ & b \\
\hline BnASSYST-275 & Duplo & 91.0 & \pm & 13.7 & $a$ & \\
\hline BnASSYST-072 & Beluga & 91.6 & \pm & 16.1 & $a$ & b \\
\hline BnASSYST-102 & Lesira & 94.2 & \pm & 24.5 & a & $b$ \\
\hline BnASSYST-238 & Drakkar & 95.9 & \pm & 14.2 & $a b$ & $b$ \\
\hline BnASSYST-110 & Olimpiade & 95.9 & \pm & 22.5 & $a$ & \\
\hline BnASSYST-217 & EVVIN & 97.9 & \pm & 23.3 & a & \\
\hline BnASSYST-373 & Rucabo & 98.5 & \pm & 35.0 & a & \\
\hline BnASSYST-520 & Ningyou 7 & 99.8 & \pm & 17.4 & a & \\
\hline BnASSYST-090 & APEX-93xGINYOU_3 & 99.9 & \pm & 13.1 & a & \\
\hline BnASSYST-002 & Remy & 101.7 & \pm & 28.4 & a & \\
\hline BnASSYST-399 & Zairai Chousenshu & 102.0 & \pm & 21.7 & $a b$ & b \\
\hline BnASSYST-045 & Fortis & 102.2 & \pm & 7.0 & a & \\
\hline BnASSYST-529 & Xiangyou 15 & 102.3 & \pm & 26.8 & a & \\
\hline BnASSYST-098 & RAFAL DH1 & 102.5 & \pm & 17.7 & a & \\
\hline BnASSYST-232 & SWU Chinese 5 & 109.3 & \pm & 23.6 & a & \\
\hline BnASSYST-436 & Tina & 113.0 & \pm & 11.3 & a & \\
\hline BnASSYST-530 & Zhongshuang II & 113.4 & \pm & 30.8 & a & \\
\hline BnASSYST-106 & SHANNON $\times$ WINNER DH LINE & 113.9 & \pm & 7.9 & a & \\
\hline BnASSYST-300 & Magma & 115.1 & \pm & 16.5 & a & \\
\hline BnASSYST-414 & SENSATION NZ & 118.4 & \pm & 13.9 & a & \\
\hline BnASSYST-230 & SWU Chinese 2 & 124.1 & \pm & 11.3 & a & \\
\hline BnASSYST-236 & SWU Chinese 9 & 128.9 & \pm & 16.9 & a & \\
\hline BnASSYST-270 & WEIHENSTEPHANER & 133.9 & \pm & 15.3 & a & \\
\hline BnASSYST-229 & SWU Chinese 1 & 144.9 & \pm & 28.5 & a & \\
\hline
\end{tabular}


Tab. 3A Lesion length and disease score of stem and hypocotyl of oilseed rape genotypes Loras and Zhongyou 821, 7 days post inoculation with S. sclerotiorum compared to control plants. Mean values and standard errors are shown.

\begin{tabular}{|c|c|c|c|c|c|}
\hline \multirow{2}{*}{ Genotype } & \multirow{2}{*}{ Inoc } & \multicolumn{2}{|c|}{ Lesion length [mm] } & \multicolumn{2}{|l|}{ Disease score } \\
\hline & & Stem & Hypocotyl & Stem & Hypocotyl \\
\hline \multirow[t]{2}{*}{ Loras } & $\mathrm{Ctl}$ & \pm 0.4 & $0.7 \pm 0.0$ & $1.0 \pm 0.0$ & $1.0 \pm 0.0$ \\
\hline & Sc & \pm 4.9 & $18.8 \pm 3.4$ & \pm 0.6 & \pm 0.5 \\
\hline Zhongyou & $\mathrm{Ctl}$ & \pm 0.3 & $1.4 \pm 0.4$ & \pm 0.0 & \pm 0.0 \\
\hline 821 & Sc & \pm 1.2 & $8.0 \pm 1.9$ & \pm 0.3 & \pm 0.2 \\
\hline
\end{tabular}

Tab. 4A Lesion length and disease score of stem and hypocotyl of oilseed rape genotypes Loras and Zhongyou 821, 7 days post inoculation with S. sclerotiorum compared to control plants. Mean values and standard errors are shown.

\begin{tabular}{|c|c|c|c|c|c|}
\hline \multirow{2}{*}{ Genotype } & \multirow{2}{*}{ Inoc } & \multicolumn{2}{|c|}{ Lesion length [mm] } & \multicolumn{2}{|l|}{ Disease score } \\
\hline & & Stem & Hypocotyl & Stem & Hypocotyl \\
\hline \multirow[t]{2}{*}{ Loras } & $\mathrm{Ctl}$ & \pm 1.4 & $1.3 \pm 0.7$ & $1.0 \pm 0.0$ & $1.0 \pm 0.0$ \\
\hline & Sc & $51.6 \pm 31.8$ & $32.5 \pm 6.7$ & $7.2 \pm 0.5$ & \pm 0.0 \\
\hline Zhongyou & $\mathrm{Ctl}$ & $2.6 \pm 1.4$ & $1.3 \pm 0.4$ & $1.0 \pm 0.0$ & \pm 0.0 \\
\hline 821 & Sc & $63.2 \pm 20.2$ & $24.8 \pm 8.1$ & $8.2 \pm 0.2$ & \pm 0.1 \\
\hline
\end{tabular}

Tab. 5A Lesion length and disease score of stem and root of oilseed rape genotypes Loras and Zhongyou 821, 7 days post inoculation with S. sclerotiorum compared to control plants. Mean values and standard errors are shown.

\begin{tabular}{|c|c|c|c|c|c|c|c|}
\hline \multirow{2}{*}{ Genotype } & \multirow{2}{*}{ Inoc } & \multicolumn{3}{|c|}{ Lesion length [mm] } & \multicolumn{3}{|l|}{ Disease score } \\
\hline & & Stem & Root & & Stem & Root & \\
\hline \multirow[t]{2}{*}{ Loras } & $\mathrm{Ctl}$ & $2.5 \pm 0.5$ & 0.9 & \pm 0.1 & $1.0 \pm 0.0$ & 1.0 & \pm 0.0 \\
\hline & Sc & $29.3 \pm 7.5$ & 13.8 & \pm 2.6 & $5.6 \pm 1.0$ & 6.1 & \pm 0.5 \\
\hline Zhongyou & $\mathrm{Ctl}$ & $2.0 \pm 0.3$ & 0.7 & \pm 0.1 & $1.0 \pm 0.0$ & 1.0 & \pm 0.0 \\
\hline 821 & Sc & $27.2 \pm 5.8$ & 16.2 & \pm 3.1 & $5.3 \pm 0.7$ & 5.9 & \pm 0.6 \\
\hline
\end{tabular}

Tab. 6A Lesion length and disease score of stem and root of oilseed rape genotypes Loras and Zhongyou 821, 7 days post inoculation with S. sclerotiorum compared to control plants. Mean values and standard errors are shown.

\begin{tabular}{|c|c|c|c|c|c|c|c|c|}
\hline \multirow{2}{*}{ Genotype } & \multirow{2}{*}{ Inoc } & \multicolumn{4}{|c|}{ Lesion length [mm] } & \multicolumn{3}{|l|}{ Disease score } \\
\hline & & Stem & & Root & & Stem & Root & \\
\hline \multirow[t]{2}{*}{ Loras } & $\mathrm{CtI}$ & 1.0 & \pm 0.3 & 2.1 & \pm 0.7 & $1.0 \pm 0.0$ & 1.0 & \pm 0.0 \\
\hline & Sc & 35.1 & \pm 11.7 & 9.5 & \pm 3.3 & $7.3 \pm 0.7$ & 5.0 & \pm 0.6 \\
\hline Zhongyou & $\mathrm{Ctl}$ & 1.2 & \pm 0.4 & 0.9 & \pm 0.3 & $1.0 \pm 0.0$ & 1.0 & \pm 0.0 \\
\hline 821 & Sc & 38.8 & \pm 12.9 & 18.4 & \pm 6.1 & $6.4 \pm 0.9$ & 6.3 & \pm 0.7 \\
\hline
\end{tabular}


Tab. 7A Conjugated phenolic acids [ $\mu \mathrm{g} / \mathrm{g} \mathrm{DW}]$ in stem and hypocotyl of oilseed rape genotypes Loras and Zhongyou 821 at 1, 3 and 7 days post inoculation with S. sclerotiorum compared to control samples. Mean values and standard deviations are shown.

\begin{tabular}{|c|c|c|c|c|c|c|c|c|c|c|c|c|c|c|c|c|c|c|c|c|c|c|c|c|c|}
\hline \multirow{2}{*}{$\begin{array}{l}\text { Phe } \\
\text { t-CA }\end{array}$} & \multirow{2}{*}{$\begin{array}{l}\text { dpi } \\
1\end{array}$} & \multicolumn{3}{|c|}{$\begin{array}{l}\text { Loras } \\
\text { Stem } \\
\text { Control }\end{array}$} & \multicolumn{3}{|c|}{ Inoculated } & \multicolumn{3}{|c|}{$\begin{array}{l}\text { Hypocotyl } \\
\text { Control }\end{array}$} & \multicolumn{3}{|c|}{ Inoculated } & $\begin{array}{l}\text { Zhon } \\
\text { Stem } \\
\text { Cont }\end{array}$ & syo & 821 & Inoce & lat & & $\begin{array}{l}\text { Hypoc } \\
\text { Contro }\end{array}$ & & & \multicolumn{3}{|c|}{ Inoculated } \\
\hline & & 0.00 & \pm & 0.00 & 0.00 & \pm & 0.00 & 0.01 & \pm & 0.01 & 0.00 & \pm & 0.00 & 0.00 & \pm & 0.01 & 0.00 & \pm & 0.00 & 0.01 & \pm & 0.01 & 0.00 & \pm & 0.00 \\
\hline & 7 & 0.00 & \pm & 0.00 & 0.05 & \pm & 0.04 & 0.01 & \pm & 0.02 & 0.07 & \pm & 0.05 & 0.02 & \pm & 0.02 & 0.05 & \pm & 0.04 & 0.02 & \pm & 0.04 & 0.05 & \pm & 0.07 \\
\hline \multirow[t]{2}{*}{ SA } & 1 & 0.00 & \pm & 0.00 & 0.00 & \pm & 0.00 & 0.00 & \pm & 0.00 & 0.00 & \pm & 0.00 & 0.00 & \pm & 0.00 & 0.00 & \pm & 0.00 & 0.00 & \pm & 0.00 & 0.00 & \pm & 0.00 \\
\hline & 3 & 0.00 & \pm & 0.00 & 0.00 & \pm & 0.00 & 0.00 & \pm & 0.00 & 0.00 & \pm & 0.00 & 0.00 & \pm & 0.00 & 0.00 & \pm & 0.00 & 0.00 & \pm & 0.00 & 0.00 & \pm & 0.00 \\
\hline \multirow{2}{*}{ p-CA } & 3 & 3.14 & \pm & 0.62 & 6.07 & \pm & 3.48 & 9.13 & \pm & 2.21 & 7.26 & \pm & 2.70 & 4.61 & \pm & 3.40 & 3.48 & \pm & 2.69 & 6.64 & \pm & 3.04 & 7.95 & \pm & 3.61 \\
\hline & 7 & 4.13 & \pm & 2.45 & 7.30 & \pm & 2.76 & 7.87 & \pm & 3.69 & 10.40 & \pm & 3.74 & 6.21 & \pm & 3.18 & 4.07 & \pm & 2.66 & 11.91 & \pm & 0.88 & 3.37 & \pm & 4.76 \\
\hline \multirow[t]{3}{*}{ ChA } & 1 & 1.05 & \pm & 0.46 & 1.44 & \pm & 1.22 & 1.09 & \pm & 0.49 & 0.78 & \pm & 0.46 & 0.55 & \pm & 0.19 & 0.35 & \pm & 0.19 & 0.73 & \pm & 0.19 & 0.74 & \pm & 0.15 \\
\hline & 3 & 0.58 & \pm & 0.13 & 0.23 & \pm & 0.28 & 0.80 & \pm & 0.44 & 0.43 & \pm & 0.25 & 0.55 & \pm & 0.42 & 0.26 & \pm & 0.25 & 0.65 & \pm & 0.19 & 0.62 & \pm & 0.20 \\
\hline & 7 & 0.61 & \pm & 0.31 & 0.57 & \pm & 0.25 & 0.77 & \pm & 0.46 & 0.79 & \pm & 0.34 & 0.56 & \pm & 0.34 & 0.88 & \pm & 1.12 & 1.02 & \pm & 0.29 & 0.61 & \pm & 0.86 \\
\hline CA & 1 & 0.48 & \pm & 0.24 & 0.47 & \pm & 0.12 & 0.03 & \pm & 0.02 & 0.06 & \pm & 0.04 & 0.16 & \pm & 0.05 & 0.20 & \pm & 0.10 & 0.02 & \pm & 0.01 & 0.01 & \pm & 0.01 \\
\hline \multirow{2}{*}{ FA } & 3 & 2.42 & \pm & 0.28 & 1.37 & \pm & 0.79 & 2.07 & \pm & 0.53 & 1.05 & \pm & 0.54 & 1.46 & \pm & 0.61 & 1.47 & \pm & 0.67 & 1.07 & \pm & 0.54 & 1.25 & \pm & 0.66 \\
\hline & 7 & 1.86 & \pm & 1.40 & 1.92 & \pm & 0.73 & 1.70 & \pm & 1.16 & 1.49 & \pm & 0.68 & 1.93 & \pm & 0.60 & 2.34 & \pm & 0.56 & 1.95 & \pm & 0.53 & 0.64 & \pm & 0.90 \\
\hline \multirow[t]{3}{*}{ SiA } & 1 & 5.57 & \pm & 0.95 & 4.27 & \pm & 1.39 & 0.11 & \pm & 0.05 & 0.25 & \pm & 0.35 & 2.16 & \pm & 1.19 & 1.66 & \pm & 0.93 & 0.23 & \pm & 0.11 & 0.16 & \pm & 0.12 \\
\hline & 3 & 4.30 & \pm & 0.76 & 2.40 & \pm & 1.28 & 0.14 & \pm & 0.12 & 0.05 & \pm & 0.07 & 2.07 & \pm & 1.47 & 2.28 & \pm & 1.09 & 0.13 & \pm & 0.08 & 0.15 & \pm & 0.08 \\
\hline & 7 & 3.42 & \pm & 2.69 & 2.68 & \pm & 1.11 & 0.08 & \pm & 0.06 & 0.12 & \pm & 0.14 & 3.28 & \pm & 1.32 & 3.64 & \pm & 1.46 & 0.35 & \pm & 0.10 & 0.05 & \pm & 0.07 \\
\hline
\end{tabular}

Phe - phenolic acids, dpi - day post inoculation, t-CA - $t$-cinnamic acid, SA - salicylic acid, p-CA - p-coumaric acid, ChA - chlorogenic acid, CA - caffeic acid, FA - ferulic acid, SiA - sinapic acid 
Tab. 8A Free phenolic acids [ $\mu \mathrm{g} / \mathrm{g}$ DW] in stem and hypocotyl of oilseed rape genotypes Loras and Zhongyou 821 at 1,3 and 7 days post inoculation with S. sclerotiorum compared to control samples. Mean values and standard deviations are shown.

\begin{tabular}{|c|c|c|c|c|c|c|c|c|c|c|c|c|c|c|c|c|c|c|c|c|c|c|c|c|c|}
\hline \multirow{4}{*}{$\begin{array}{l}\text { Phe } \\
\text { t-CA }\end{array}$} & \multirow{4}{*}{$\begin{array}{l}\text { dpi } \\
1\end{array}$} & \multicolumn{12}{|c|}{ Loras } & \multicolumn{12}{|c|}{ Zhongyou 821} \\
\hline & & \multicolumn{6}{|c|}{ Stem } & \multicolumn{6}{|c|}{ Hypocotyl } & \multicolumn{6}{|c|}{ Stem } & \multicolumn{6}{|c|}{ Hypocotyl } \\
\hline & & \multicolumn{3}{|c|}{ Control } & \multicolumn{3}{|c|}{ Inoculated } & \multicolumn{3}{|c|}{ Control } & \multicolumn{3}{|c|}{ Inoculated } & \multicolumn{3}{|c|}{ Control } & \multicolumn{3}{|c|}{ Inoculated } & \multicolumn{3}{|c|}{ Control } & \multicolumn{3}{|c|}{ Inoculated } \\
\hline & & 0.01 & \pm & 0.02 & 0.01 & \pm & 0.01 & 0.01 & \pm & 0.02 & 0.04 & \pm & 0.04 & 0.00 & \pm & 0.00 & 0.00 & \pm & 0.00 & 0.00 & \pm & 0.00 & 0.00 & \pm & 0.00 \\
\hline & 3 & 0.01 & \pm & 0.03 & 0.09 & \pm & 0.06 & 0.03 & \pm & 0.04 & 0.09 & \pm & 0.08 & 0.00 & \pm & 0.00 & 0.23 & \pm & 0.14 & 0.00 & \pm & 0.00 & 0.49 & \pm & 0.83 \\
\hline & 7 & 0.02 & \pm & 0.02 & 0.42 & \pm & 0.16 & 0.03 & \pm & 0.04 & 0.58 & \pm & 0.54 & 0.01 & \pm & 0.01 & 1.06 & \pm & 1.06 & 0.00 & \pm & 0.00 & 1.98 & \pm & 2.33 \\
\hline \multirow[t]{3}{*}{ SA } & 1 & 0.02 & \pm & 0.02 & 0.02 & \pm & 0.02 & 0.02 & \pm & 0.02 & 0.03 & \pm & 0.04 & 0.00 & \pm & 0.00 & 0.00 & \pm & 0.00 & 0.04 & \pm & 0.05 & 0.05 & \pm & 0.09 \\
\hline & 3 & 0.01 & \pm & 0.01 & 0.02 & \pm & 0.04 & 0.03 & \pm & 0.02 & 0.04 & \pm & 0.05 & 0.01 & \pm & 0.01 & 0.03 & \pm & 0.03 & 0.05 & \pm & 0.06 & 0.05 & \pm & 0.06 \\
\hline & 7 & 0.01 & \pm & 0.01 & 0.08 & \pm & 0.08 & 0.03 & \pm & 0.01 & 0.04 & \pm & 0.07 & 0.02 & \pm & 0.03 & 0.18 & \pm & 0.11 & 0.05 & \pm & 0.05 & 0.04 & \pm & 0.03 \\
\hline \multirow[t]{3}{*}{$p-C A$} & 1 & 0.61 & \pm & 0.41 & 1.20 & \pm & 0.75 & 1.12 & \pm & 0.59 & 1.37 & \pm & 0.88 & 0.80 & \pm & 0.47 & 0.76 & \pm & 0.31 & 1.29 & \pm & 0.41 & 1.82 & \pm & 1.75 \\
\hline & 3 & 0.44 & \pm & 0.08 & 1.18 & \pm & 0.91 & 1.17 & \pm & 1.31 & 2.02 & \pm & 2.91 & 0.83 & \pm & 0.28 & 0.81 & \pm & 0.19 & 1.75 & \pm & 1.22 & 1.16 & \pm & 0.22 \\
\hline & 7 & 0.74 & \pm & 0.30 & 0.66 & \pm & 0.32 & 1.07 & \pm & 0.68 & 0.95 & \pm & 0.82 & 1.14 & \pm & 0.57 & 1.27 & \pm & 0.38 & 0.94 & \pm & 0.04 & 3.10 & \pm & 3.78 \\
\hline \multirow[t]{3}{*}{ ChA } & 1 & 0.73 & \pm & 0.35 & 2.34 & \pm & 2.26 & 0.79 & \pm & 0.28 & 1.35 & \pm & 0.65 & 1.15 & \pm & 0.63 & 1.26 & \pm & 0.66 & 1.11 & \pm & 0.39 & 1.10 & \pm & 0.43 \\
\hline & 3 & 0.59 & \pm & 0.32 & 2.87 & \pm & 2.00 & 1.34 & \pm & 0.39 & 1.53 & \pm & 0.69 & 1.49 & \pm & 0.56 & 1.96 & \pm & 0.99 & 0.94 & \pm & 0.39 & 2.83 & \pm & 1.88 \\
\hline & 7 & 1.42 & \pm & 0.71 & 3.64 & \pm & 1.29 & 1.09 & \pm & 0.20 & 3.59 & \pm & 1.40 & 2.59 & \pm & 2.17 & 8.70 & \pm & 4.84 & 1.28 & \pm & 0.23 & 1.42 & \pm & 0.19 \\
\hline \multirow[t]{3}{*}{ CA } & 1 & 0.10 & \pm & 0.17 & 0.05 & \pm & 0.00 & 0.04 & \pm & 0.04 & 0.03 & \pm & 0.01 & 0.03 & \pm & 0.01 & 0.11 & \pm & 0.07 & 0.01 & \pm & 0.01 & 0.08 & \pm & 0.13 \\
\hline & 3 & 0.02 & \pm & 0.01 & 0.19 & \pm & 0.07 & 0.04 & \pm & 0.02 & 0.47 & \pm & 0.54 & 0.04 & \pm & 0.00 & 0.29 & \pm & 0.12 & 0.06 & \pm & 0.06 & 0.23 & \pm & 0.12 \\
\hline & 7 & 0.03 & \pm & 0.03 & 0.42 & \pm & 0.14 & 0.04 & \pm & 0.01 & 0.46 & \pm & 0.09 & 0.12 & \pm & 0.11 & 0.70 & \pm & 0.10 & 0.02 & \pm & 0.00 & 0.31 & \pm & 0.18 \\
\hline \multirow[t]{3}{*}{ FA } & 1 & 0.19 & \pm & 0.19 & 0.25 & \pm & 0.19 & 0.14 & \pm & 0.03 & 0.17 & \pm & 0.11 & 0.09 & \pm & 0.03 & 0.16 & \pm & 0.10 & 0.14 & \pm & 0.05 & 0.18 & \pm & 0.07 \\
\hline & 3 & 0.86 & \pm & 0.40 & 0.27 & \pm & 0.08 & 0.21 & \pm & 0.10 & 0.24 & \pm & 0.10 & 0.10 & \pm & 0.02 & 0.29 & \pm & 0.07 & 0.10 & \pm & 0.05 & 0.34 & \pm & 0.09 \\
\hline & 7 & 0.42 & \pm & 0.15 & 0.53 & \pm & 0.29 & 0.18 & \pm & 0.03 & 0.34 & \pm & 0.16 & 0.19 & \pm & 0.08 & 2.08 & \pm & 3.09 & 0.17 & \pm & 0.03 & 0.41 & \pm & 0.16 \\
\hline \multirow[t]{3}{*}{ SiA } & 1 & 0.38 & \pm & 0.12 & 0.76 & \pm & 0.77 & 0.05 & \pm & 0.06 & 0.14 & \pm & 0.09 & 0.39 & \pm & 0.23 & 1.06 & \pm & 1.10 & 0.34 & \pm & 0.09 & 0.20 & \pm & 0.11 \\
\hline & 3 & 0.30 & \pm & 0.05 & 0.48 & \pm & 0.07 & 0.10 & \pm & 0.08 & 0.07 & \pm & 0.06 & 0.57 & \pm & 0.15 & 0.63 & \pm & 0.22 & 0.20 & \pm & 0.12 & 0.10 & \pm & 0.07 \\
\hline & 7 & 0.32 & \pm & 0.13 & 1.06 & \pm & 0.94 & 0.16 & \pm & 0.02 & 0.26 & \pm & 0.19 & 0.53 & \pm & 0.34 & 1.28 & \pm & 1.00 & 0.38 & \pm & 0.03 & 0.36 & \pm & 0.46 \\
\hline
\end{tabular}

Phe - phenolic acids, dpi - day post inoculation, t-CA - t-cinnamic acid, SA - salicylic acid, p-CA - p-coumaric acid, ChA - chlorogenic acid, CA - caffeic acid, FA - ferulic acid, SiA - sinapic acid 
Tab. 9A Ratio of peroxidase activity between control and inoculated plants in stem and hypocotyl of the oilseed rape genotypes Loras and Zhongyou 821 at 1, 3, 5 and 7 days post inoculation with S. sclerotiorum. Mean values and standard errors are shown.

\begin{tabular}{|c|c|c|c|c|c|}
\hline \multirow[b]{2}{*}{ dpi } & \multicolumn{2}{|l|}{ Loras } & \multicolumn{3}{|c|}{ Zhongyou 821} \\
\hline & Stem & & Hypocotyl & Stem & Hypocotyl \\
\hline 1 & 1.21 & \pm 0.16 & $1.55 \pm 0.20$ & $0.75 \pm 0.10$ & $1.00 \pm 0.21$ \\
\hline 3 & 0.88 & \pm 0.11 & $1.88 \pm 0.15$ & \pm 0.10 & \pm 0.04 \\
\hline 5 & 0.78 & \pm 0.07 & $2.15 \pm 0.06$ & \pm 0.10 & \pm 0.17 \\
\hline 7 & 1.20 & \pm 0.03 & $1.65 \pm 0.10$ & $0.91 \pm 0.10$ & $6.33 \pm 0.12$ \\
\hline
\end{tabular}

Tab. 10A Ratio of peroxidase activity between control and inoculated plants in stem and hypocotyl of the oilseed rape genotypes Loras and Zhongyou 821 at 1, 3, 5 and 7 days post inoculation with S. sclerotiorum. Mean values and standard errors are shown.

\begin{tabular}{|c|c|c|c|c|c|c|}
\hline \multirow[b]{2}{*}{ dpi } & \multicolumn{2}{|l|}{ Loras } & \multicolumn{4}{|c|}{ Zhongyou 821} \\
\hline & Stem & & Hypoc & otyl & Stem & Hypocotyl \\
\hline 1 & 0.88 & \pm 0.11 & 0.79 & \pm 0.12 & $0.59 \pm 0.10$ & $0.82 \pm 0.09$ \\
\hline 3 & 0.78 & \pm 0.18 & 0.87 & \pm 0.11 & $0.88 \pm 0.03$ & \pm 0.08 \\
\hline 5 & 0.93 & \pm 0.16 & 0.75 & \pm 0.08 & \pm 0.24 & \pm 0.06 \\
\hline 7 & 0.89 & \pm 0.07 & 0.95 & \pm 0.10 & $2.25 \pm 0.23$ & \pm 0.03 \\
\hline
\end{tabular}

Tab. 11A Ratio of peroxidase activity between control and inoculated plants in stem and root of the oilseed rape genotypes Loras and Zhongyou 821 at 1, 4 and 7 days post inoculation with S. sclerotiorum. Mean values and standard errors are shown.

\begin{tabular}{lllllllllllll}
\hline & \multicolumn{3}{c}{$\begin{array}{l}\text { Loras } \\
\text { dpi }\end{array}$} & Stem & & \multicolumn{1}{c}{ Root } & \multicolumn{9}{c}{ Zhongyou 821 } \\
\hline 1 & 0.98 & \pm & 0.07 & 0.96 & \pm & 0.13 & 1.53 & \pm & 0.29 & 0.72 & \pm & 0.05 \\
4 & 1.34 & \pm & 0.13 & 1.33 & \pm & 0.15 & 1.69 & \pm & 0.80 & 0.70 & \pm & 0.12 \\
7 & 1.40 & \pm & 0.08 & 2.32 & \pm & 0.17 & 1.42 & \pm & 0.21 & 1.58 & \pm & 0.30 \\
\hline
\end{tabular}




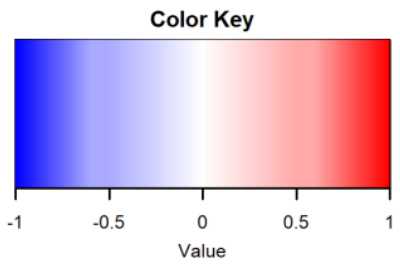

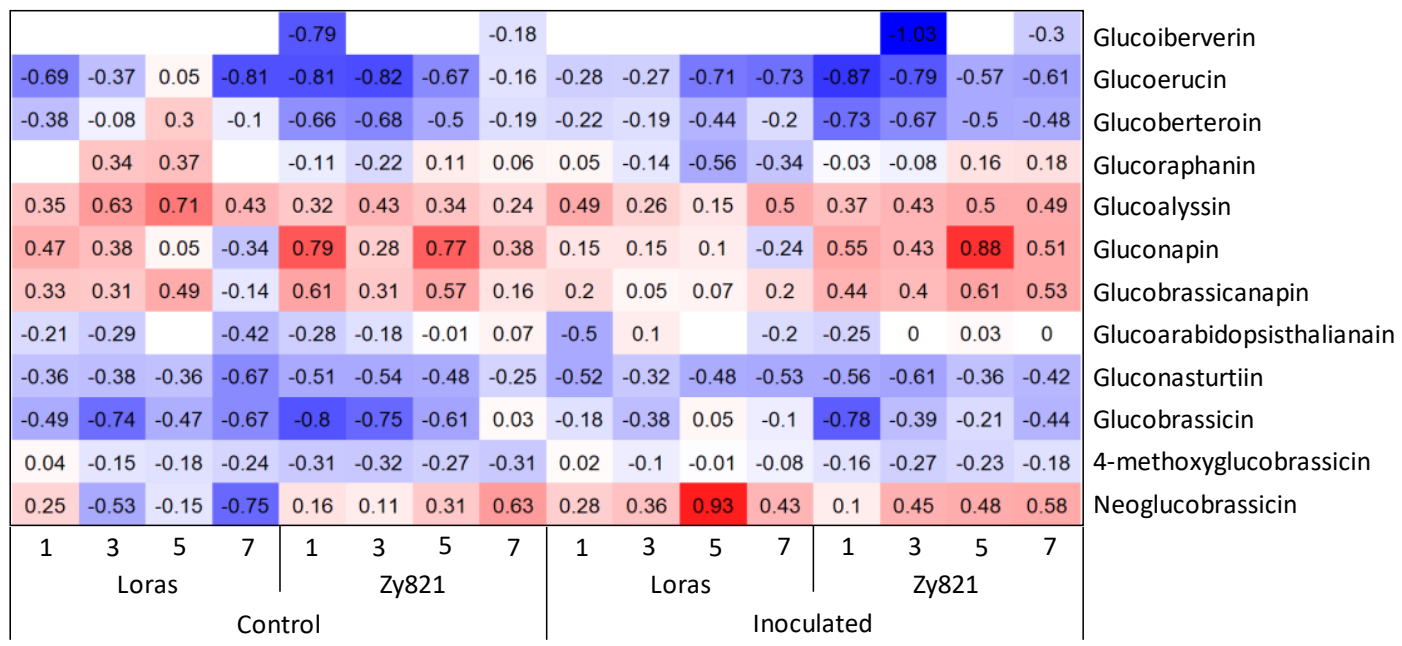

Fig. 1A Relation of glucosinolates in stem compared to hypocotyl of non-inoculated and inoculated oilseed rape genotypes Loras and Zhongyou 821 (Zy821) at 1, 3, 5 and $7 \mathrm{dpi}$ with S. sclerotiorum.

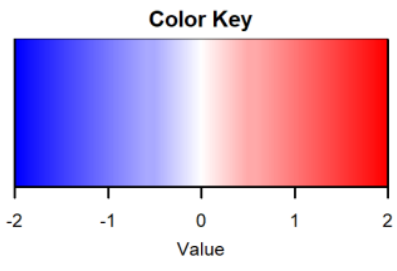

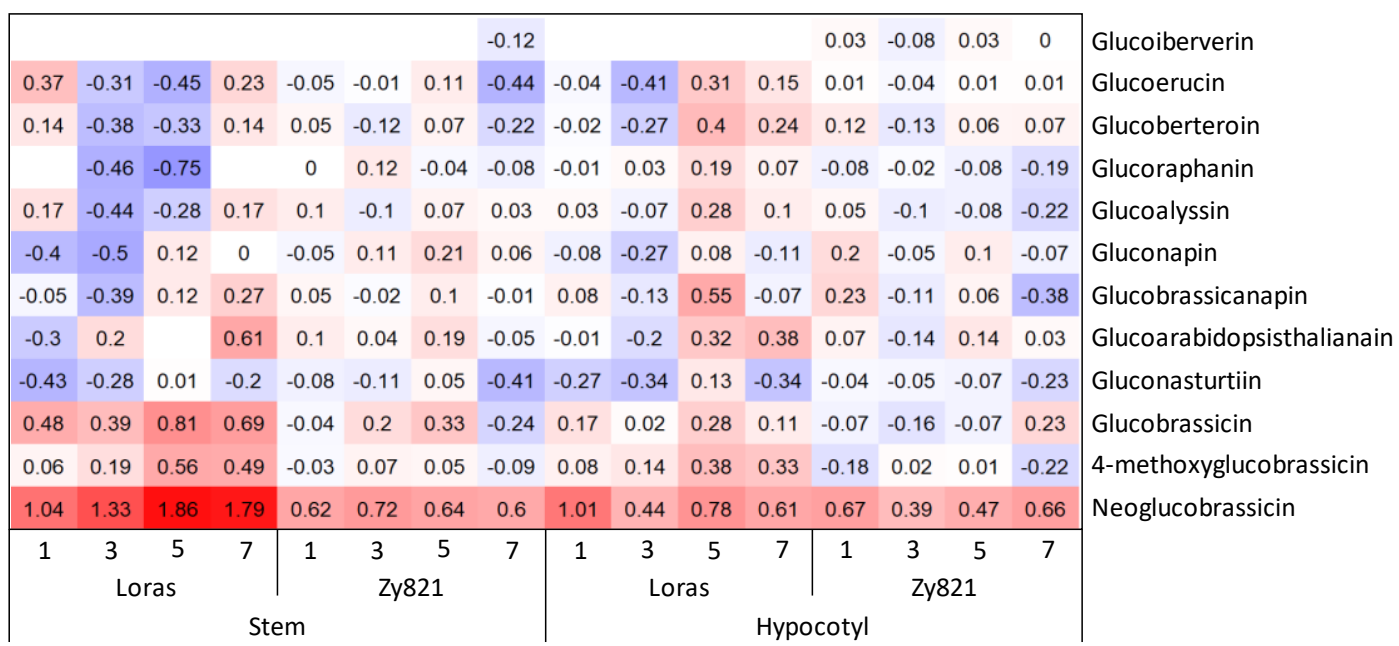

Fig. 2A Effect of inoculation with S. sclerotiorum on glucosinolates in stem and hypocotyl of the oilseed rape genotypes Loras and Zhongyou 821 (Zy821) at 1, 3, 5 and $7 \mathrm{dpi}$. 
Tab. 12A Glucosinolates [nmol/g FW] in control and inoculated plants in stem and hypocotyl of the oilseed rape genotypes Loras and Zhongyou 821 at 7 days post inoculation with S. sclerotiorum. Mean values and standard deviations are shown.

\begin{tabular}{|c|c|c|c|c|c|c|c|c|c|c|c|c|c|}
\hline \multirow{3}{*}{$\begin{array}{l}\text { Genotype } \\
\text { Loras }\end{array}$} & \multirow{3}{*}{$\begin{array}{l}\text { GSL } \\
\text { Glucoiberverin }\end{array}$} & \multicolumn{6}{|l|}{ Stem } & \multicolumn{6}{|c|}{ Hypocotyl } \\
\hline & & \multicolumn{3}{|l|}{ Control } & \multicolumn{3}{|c|}{ Inoculated } & \multicolumn{3}{|l|}{ Control } & \multicolumn{3}{|c|}{ Inoculated } \\
\hline & & 0.00 & \pm & 0.00 & 0.00 & \pm & 0.00 & 0.00 & \pm & 0.00 & 0.00 & \pm & 0.00 \\
\hline & Glucoerucin & 6.54 & \pm & 4.72 & 11.23 & \pm & 5.31 & 42.31 & \pm & 17.50 & 60.31 & \pm & 52.91 \\
\hline & Glucoberteroin & 94.35 & \pm & 62.48 & 131.37 & \pm & 64.92 & 119.35 & \pm & 69.32 & 209.41 & \pm & 104.28 \\
\hline & Glucoiberin & 0.00 & \pm & 0.00 & 0.00 & \pm & 0.00 & 0.00 & \pm & 0.00 & 0.00 & \pm & 0.00 \\
\hline & Glucoraphanin & 0.00 & \pm & 0.00 & 2.39 & \pm & 2.75 & 4.49 & \pm & 1.49 & 5.27 & \pm & 4.02 \\
\hline & Glucoalyssin & 49.06 & \pm & 22.02 & 72.75 & \pm & 32.74 & 18.44 & \pm & 8.63 & 23.09 & \pm & 11.71 \\
\hline & Gluconapin & 20.49 & \pm & 21.68 & 20.28 & \pm & 15.07 & 45.15 & \pm & 38.58 & 35.13 & \pm & 31.74 \\
\hline & Glucobrassicanapin & 53.03 & \pm & 68.70 & 99.29 & \pm & 55.93 & 73.90 & \pm & 71.05 & 62.87 & \pm & 39.02 \\
\hline & Glucoarabidopsisthalianain & 6.77 & \pm & 13.55 & 27.57 & \pm & 20.86 & 18.01 & \pm & 8.61 & 43.43 & \pm & 28.14 \\
\hline & Gluconasturtiin & 190.18 & \pm & 175.26 & 120.26 & \pm & 76.95 & 881.79 & \pm & 479.28 & 404.41 & \pm & 176.59 \\
\hline & Glucobrassicin & 41.94 & \pm & 7.76 & 204.44 & \pm & 17.66 & 197.95 & \pm & 46.16 & 256.69 & \pm & 86.73 \\
\hline & 4-methoxyglucobrassicin & 31.75 & \pm & 11.15 & 96.98 & \pm & 12.15 & 54.92 & \pm & 13.05 & 117.44 & \pm & 41.57 \\
\hline & Neoglucobrassicin & 100.42 & \pm & 50.56 & $1,531.69$ & \pm & 828.69 & $2,238.15$ & \pm & $1,192.39$ & $2,300.45$ & \pm & $1,805.53$ \\
\hline \multirow[t]{13}{*}{ Zhongyou 821} & Glucoiberverin & 2.71 & \pm & 5.4 & 2.1 & \pm & 4.1 & 4.1 & \pm & 4.8 & 4.1 & \pm & 2.2 \\
\hline & Glucoerucin & 747.53 & \pm & $1,130.5$ & 271.9 & \pm & 153.8 & $1,081.1$ & \pm & $1,124.6$ & $1,105.2$ & \pm & 278.4 \\
\hline & Glucoberteroin & $1,472.44$ & \pm & $1,673.9$ & 879.4 & \pm & 214.8 & $2,263.1$ & \pm & $2,397.3$ & $2,681.8$ & \pm & $1,386.9$ \\
\hline & Glucoiberin & 0.00 & \pm & 0.0 & 0.0 & \pm & 0.0 & 0.0 & \pm & 0.0 & 0.0 & \pm & 0.0 \\
\hline & Glucoraphanin & 104.51 & \pm & 65.9 & 87.8 & \pm & 40.4 & 91.1 & \pm & 65.5 & 58.5 & \pm & 20.8 \\
\hline & Glucoalyssin & 771.71 & \pm & 174.5 & 830.6 & \pm & 188.7 & 445.8 & \pm & 366.1 & 266.8 & \pm & 138.4 \\
\hline & Gluconapin & $1,061.83$ & \pm & 468.3 & $1,211.9$ & \pm & 625.1 & 440.7 & \pm & 546.0 & 372.0 & \pm & 267.6 \\
\hline & Glucobrassicanapin & $1,457.36$ & \pm & 907.4 & $1,424.6$ & \pm & 500.0 & $1,009.7$ & \pm & 695.8 & 421.7 & \pm & 307.6 \\
\hline & Glucoarabidopsisthalianain & 396.05 & \pm & 226.9 & 351.9 & \pm & 116.1 & 334.0 & \pm & 226.4 & 355.7 & \pm & 182.2 \\
\hline & Gluconasturtiin & $3,369.00$ & \pm & $3,526.1$ & $1,312.6$ & \pm & 455.8 & $5,948.1$ & \pm & $6,357.2$ & $3,488.4$ & \pm & 716.2 \\
\hline & Glucobrassicin & 191.70 & \pm & 197.2 & 111.1 & \pm & 5.2 & 180.5 & \pm & 181.8 & 303.2 & \pm & 148.7 \\
\hline & 4-methoxyglucobrassicin & 92.77 & \pm & 30.9 & 75.3 & \pm & 11.1 & 191.3 & \pm & 184.1 & 114.0 & \pm & 32.7 \\
\hline & Neoglucobrassicin & $2,974.62$ & \pm & $2,090.4$ & $2,986.8$ & \pm & 825.8 & $2,773.5$ & \pm & $3,413.4$ & $3,161.4$ & \pm & $1,490.9$ \\
\hline
\end{tabular}




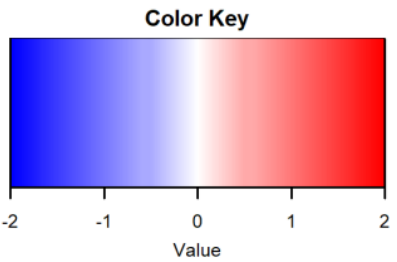

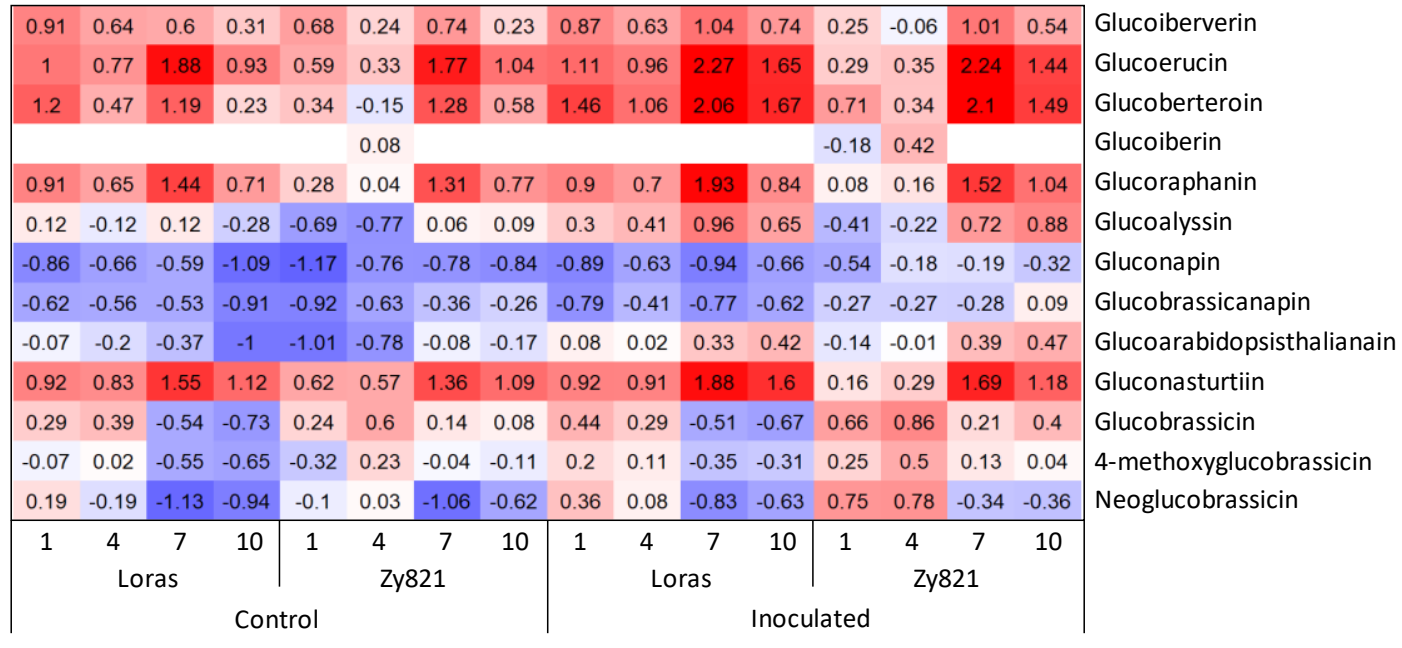

Fig. 3A Relation of glucosinolates in stem compared to root of non-inoculated and inoculated oilseed rape genotypes Loras and Zhongyou 821 (Zy821) at 1, 4, 7 and 10 dpi with S. sclerotiorum.

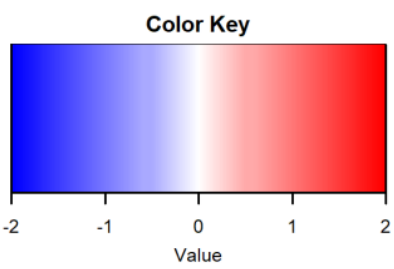

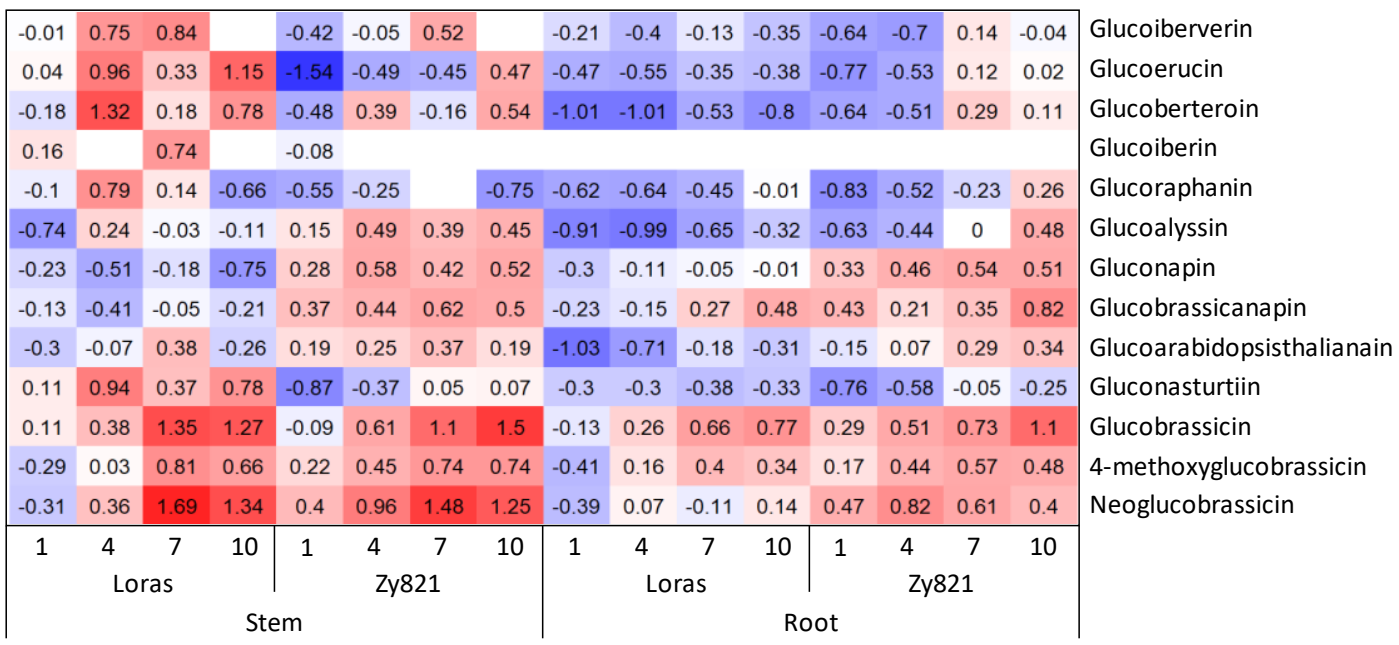

Fig. 4A Effect of inoculation with S. sclerotiorum on glucosinolates in stem and root of the oilseed rape genotypes Loras and Zhongyou 821 (Zy821) at 1, 4, 7 and $10 \mathrm{dpi}$. 
Tab. 13A Glucosinolates [nmol/g FW] in control and inoculated plants in stem and root of the oilseed rape genotypes Loras and Zhongyou 821 at 7 days post inoculation with S. sclerotiorum. Mean values and standard deviations are shown.

\begin{tabular}{|c|c|c|c|c|c|c|c|c|c|c|c|c|c|}
\hline \multirow[b]{2}{*}{ Genotype } & \multirow[b]{2}{*}{ GSL } & \multicolumn{6}{|l|}{ Stem } & \multicolumn{6}{|l|}{ Hypocotyl } \\
\hline & & Control & & & Inoculated & & & Control & & & Inoculated & & \\
\hline \multirow[t]{13}{*}{ Loras } & Glucoiberverin & 11.61 & \pm & 6.62 & 26.77 & \pm & 11.64 & 13.54 & \pm & 12.40 & 57.04 & \pm & 20.25 \\
\hline & Glucoerucin & 15.59 & \pm & 1.11 & $1,234.71$ & \pm & $1,044.44$ & $1,905.78$ & \pm & $1,785.16$ & 0.00 & \pm & 0.00 \\
\hline & Glucoberteroin & 56.71 & \pm & 6.87 & $1,136.48$ & \pm & 733.03 & $1,262.08$ & \pm & $1,066.58$ & 54.67 & \pm & 21.56 \\
\hline & Glucoiberin & 105.68 & \pm & 68.67 & 14.39 & \pm & 24.92 & 0.00 & \pm & 0.00 & 0.00 & \pm & 0.00 \\
\hline & Glucoraphanin & 5.30 & \pm & 4.86 & 125.18 & \pm & 116.47 & 110.76 & \pm & 102.38 & $1,785.29$ & \pm & 454.24 \\
\hline & Glucoalyssin & 99.25 & \pm & 82.70 & 241.03 & \pm & 92.77 & 187.20 & \pm & 38.02 & $2,066.22$ & \pm & 412.33 \\
\hline & Gluconapin & 77.40 & \pm & 12.32 & 376.63 & \pm & 507.77 & 99.80 & \pm & 28.28 & 69.91 & \pm & 27.96 \\
\hline & Glucobrassicanapin & 312.77 & \pm & 210.67 & 994.10 & \pm & 813.46 & 354.85 & \pm & 111.59 & $35,484.75$ & \pm & $1,550.15$ \\
\hline & Glucoarabidopsisthalianain & 54.12 & \pm & 47.12 & 93.47 & \pm & 47.62 & 30.95 & \pm & 9.19 & $5,204.07$ & \pm & $1,900.86$ \\
\hline & Gluconasturtiin & 920.07 & \pm & 418.03 & $29,330.81$ & \pm & $27,288.66$ & $24,888.62$ & \pm & $20,024.82$ & 69.91 & \pm & 27.96 \\
\hline & Glucobrassicin & 412.66 & \pm & 161.86 & $1,577.02$ & \pm & $2,231.16$ & 552.21 & \pm & 81.44 & $5,904.62$ & \pm & $2,831.58$ \\
\hline & 4-methoxyglucobrassicin & 350.64 & \pm & 128.65 & 220.50 & \pm & 286.56 & 128.75 & \pm & 53.44 & $5,904.62$ & \pm & $2,831.58$ \\
\hline & Neoglucobrassicin & $1,081.61$ & \pm & 320.51 & $12,443.48$ & \pm & $18,538.27$ & $3,104.67$ & \pm & 1,193.71 & 558.98 & \pm & 130.61 \\
\hline Zhongyou & Glucoiberverin & 10.31 & \pm & 5.14 & 15.28 & \pm & 16.23 & 302.24 & \pm & 137.33 & 198.72 & \pm & 141.85 \\
\hline \multirow[t]{12}{*}{821} & Glucoerucin & 164.57 & \pm & 99.97 & $1,156.54$ & \pm & $1,991.64$ & 0.00 & \pm & 0.00 & 0.00 & \pm & 0.00 \\
\hline & Glucoberteroin & 439.82 & \pm & 165.56 & $1,218.76$ & \pm & $2,031.53$ & 310.51 & \pm & 40.06 & 235.47 & \pm & 166.43 \\
\hline & Glucoiberin & 70.12 & \pm & 38.46 & 0.00 & \pm & 0.00 & 0.00 & \pm & 0.00 & 0.00 & \pm & 0.00 \\
\hline & Glucoraphanin & 61.02 & \pm & 28.69 & 76.05 & \pm & 131.72 & $9,948.95$ & \pm & $3,362.47$ & $8,594.09$ & \pm & $5,236.18$ \\
\hline & Glucoalyssin & $1,080.75$ & \pm & 385.77 & 169.33 & \pm & 149.60 & $14,545.88$ & \pm & $2,017.24$ & $16,301.77$ & \pm & $4,983.24$ \\
\hline & Gluconapin & $1,211.76$ & \pm & 774.42 & 80.61 & \pm & 68.02 & 48.95 & \pm & 9.56 & 178.03 & \pm & 44.15 \\
\hline & Glucobrassicanapin & $1,956.87$ & \pm & 775.70 & 441.98 & \pm & 287.57 & $110,681.64$ & \pm & $8,693.34$ & $80,906.01$ & \pm & $20,442.72$ \\
\hline & Glucoarabidopsisthalianain & 534.53 & \pm & 206.91 & 132.54 & \pm & 79.08 & 341.97 & \pm & 126.03 & 7,193.35 & \pm & $2,992.11$ \\
\hline & Gluconasturtiin & $3,726.79$ & \pm & $1,833.82$ & $14,039.26$ & \pm & $23,562.57$ & 48.95 & \pm & 9.56 & 178.03 & \pm & 44.15 \\
\hline & Glucobrassicin & 552.34 & \pm & 397.47 & $2,120.80$ & \pm & $1,622.60$ & 693.24 & \pm & 97.95 & $25,548.73$ & \pm & $15,993.95$ \\
\hline & 4-methoxyglucobrassicin & 433.17 & \pm & 144.10 & 335.98 & \pm & 229.63 & 693.24 & \pm & 97.95 & $25,548.73$ & \pm & $15,993.95$ \\
\hline & Neoglucobrassicin & $7,041.50$ & \pm & $2,050.10$ & $15,109.27$ & \pm & $21,861.25$ & 207.34 & \pm & 16.84 & 671.18 & \pm & 253.17 \\
\hline
\end{tabular}


Tab. 14A Glucosinolates [nmol/g FW] in control and inoculated plants in stem and root of the oilseed rape genotypes Loras and Zhongyou 821 at 7 days post inoculation with S. sclerotiorum. Mean values and standard deviations are shown.

\begin{tabular}{|c|c|c|c|c|c|c|c|c|c|c|c|c|c|}
\hline \multirow{2}{*}{$\begin{array}{l}\text { Genotype } \\
\text { Loras }\end{array}$} & \multirow{2}{*}{$\begin{array}{l}\text { GSL } \\
\text { Glucoiberverin }\end{array}$} & \multicolumn{6}{|l|}{ Stem } & \multicolumn{6}{|l|}{ Hypocotyl } \\
\hline & & 1.11 & \pm & 1.92 & 7.52 & \pm & 7.99 & 13.35 & \pm & 11.68 & 18.49 & \pm & 10.86 \\
\hline & Glucoerucin & 42.00 & \pm & 52.95 & 60.24 & \pm & 65.35 & $2,427.61$ & \pm & 983.65 & $1,877.86$ & \pm & 671.95 \\
\hline & Glucoberteroin & 125.16 & \pm & 141.74 & 142.62 & \pm & 143.36 & $1,551.59$ & \pm & 445.27 & $1,915.86$ & \pm & $1,355.66$ \\
\hline & Glucoiberin & 18.23 & \pm & 31.57 & 49.95 & \pm & 86.52 & 0.00 & \pm & 0.00 & 0.00 & \pm & 0.00 \\
\hline & Glucoraphanin & 12.81 & \pm & 18.21 & 15.56 & \pm & 13.98 & 264.50 & \pm & 75.77 & 192.37 & \pm & 49.76 \\
\hline & Glucoalyssin & 91.49 & \pm & 96.44 & 130.48 & \pm & 55.41 & 249.37 & \pm & 82.07 & 220.39 & \pm & 57.02 \\
\hline & Gluconapin & 74.26 & \pm & 30.97 & 178.54 & \pm & 153.23 & 92.32 & \pm & 17.24 & 59.39 & \pm & 70.33 \\
\hline & Glucobrassicanapin & 235.62 & \pm & 91.58 & 684.93 & \pm & 314.99 & 408.63 & \pm & 223.53 & 596.12 & \pm & 318.58 \\
\hline & Glucoarabidopsisthalianain & 39.27 & \pm & 36.50 & 361.02 & \pm & 84.82 & 106.51 & \pm & 104.75 & 211.52 & \pm & 235.67 \\
\hline & Gluconasturtiin & 939.18 & \pm & 995.51 & $2,063.78$ & \pm & $1,759.76$ & $42,321.43$ & \pm & $11,386.10$ & $27,750.23$ & \pm & $4,981.14$ \\
\hline & Glucobrassicin & 252.60 & \pm & 165.71 & $3,643.82$ & \pm & $1,370.74$ & 435.10 & \pm & 299.04 & $2,073.24$ & \pm & 806.31 \\
\hline & 4-methoxyglucobrassicin & 90.41 & \pm & 32.39 & 561.64 & \pm & 200.26 & 85.05 & \pm & 51.33 & 275.02 & \pm & 96.73 \\
\hline & Neoglucobrassicin & 395.91 & \pm & 270.49 & $51,616.41$ & \pm & $18,782.61$ & $1,601.60$ & \pm & 620.71 & $1,843.58$ & \pm & 307.97 \\
\hline \multirow[t]{13}{*}{ Zhongyou 821} & Glucoiberverin & 1.06 & \pm & 1.83 & 3.63 & \pm & 6.28 & 36.48 & \pm & 3.00 & 34.28 & \pm & 16.34 \\
\hline & Glucoerucin & 14.29 & \pm & 15.99 & 10.09 & \pm & 8.83 & 5889.69 & \pm & $1,798.65$ & $5,516.96$ & \pm & $1,739.96$ \\
\hline & Glucoberteroin & 64.36 & \pm & 65.95 & 65.55 & \pm & 32.56 & $11,375.32$ & \pm & $4,401.18$ & $12,580.47$ & \pm & $4,311.67$ \\
\hline & Glucoiberin & 0.00 & \pm & 0.00 & 0.00 & \pm & 0.00 & 0.00 & \pm & 0.00 & 0.00 & \pm & 0.00 \\
\hline & Glucoraphanin & 9.69 & \pm & 8.16 & 0.00 & \pm & 0.00 & 814.79 & \pm & 583.56 & 314.22 & \pm & 163.29 \\
\hline & Glucoalyssin & 190.97 & \pm & 112.27 & 349.18 & \pm & 199.43 & $1,741.29$ & \pm & $1,147.03$ & 994.19 & \pm & 135.39 \\
\hline & Gluconapin & 461.74 & \pm & 200.32 & 710.21 & \pm & 333.41 & 41.05 & \pm & 22.90 & 231.84 & \pm & 94.95 \\
\hline & Glucobrassicanapin & $1,316.72$ & \pm & 388.27 & $3,258.11$ & \pm & $1,917.92$ & 233.79 & \pm & 98.22 & 714.54 & \pm & 219.18 \\
\hline & Glucoarabidopsisthalianain & 260.57 & \pm & 165.90 & 348.48 & \pm & 162.62 & 540.09 & \pm & 147.92 & 625.60 & \pm & 324.02 \\
\hline & Gluconasturtiin & 819.80 & \pm & 255.18 & 995.92 & \pm & 281.71 & $90,346.68$ & \pm & $6,819.05$ & $58,620.84$ & \pm & $18,217.87$ \\
\hline & Glucobrassicin & 75.71 & \pm & 31.48 & $2,043.69$ & \pm & $1,300.79$ & 466.08 & \pm & 168.67 & $2,437.64$ & \pm & 975.12 \\
\hline & 4-methoxyglucobrassicin & 81.72 & \pm & 21.93 & 473.39 & \pm & 164.59 & 134.49 & \pm & 34.45 & 407.71 & \pm & 106.88 \\
\hline & Neoglucobrassicin & $1,707.97$ & \pm & 277.82 & $31,920.74$ & \pm & $13,139.43$ & $3,177.90$ & \pm & 515.11 & $9,845.66$ & \pm & $2,416.57$ \\
\hline
\end{tabular}


Tab. 15A Growth parameters and lesion length 7 days post inoculation with S. sclerotiorum of the OSR-genotypes Loras and Zhongyou 821 after treatment with growth regulators or water control. Means and standard errors are shown.

\begin{tabular}{|c|c|c|c|c|c|c|}
\hline $\begin{array}{l}\text { Para- } \\
\text { meter }\end{array}$ & $\begin{array}{l}\text { Geno- } \\
\text { type }\end{array}$ & $\begin{array}{l}\text { Treatment } \\
\text { Caramba }\end{array}$ & Carax & Moddus & Toprex & Control \\
\hline \multirow{2}{*}{$\mathrm{BBCH}$} & Loras & $57.6 \pm 1.4$ & $55.0 \pm 1.5$ & $56.0 \pm 1.3$ & $58.7 \pm 1.7$ & $61.3 \pm 1.0$ \\
\hline & Zhongyou 821 & $43.6 \pm 2.0$ & $44.5 \pm 2.1$ & $43.8 \pm 1.8$ & $42.7 \pm 1.8$ & $50.8 \pm 2.1$ \\
\hline \multirow{2}{*}{$\begin{array}{l}\text { Plant } \\
\text { height } \\
{[\mathrm{mm}]}\end{array}$} & Loras & $\begin{array}{l}422.3 \pm \\
36.0\end{array}$ & $408.3 \pm 39.8$ & $435.5 \pm 41.4$ & $538.8 \pm 40.0$ & $732.3 \pm 41.2$ \\
\hline & Zhongyou 821 & $\begin{array}{l}142.9 \pm \\
16.3\end{array}$ & $174.5 \pm 23.5$ & $125.7 \pm 12.9$ & $155.7 \pm 17.1$ & $401.4 \pm 50.6$ \\
\hline \multirow{2}{*}{$\begin{array}{l}\text { Stem } \\
\varnothing \\
{[\mathrm{mm}]}\end{array}$} & Loras & $8.8 \pm 0.2$ & $8.3 \pm 0.2$ & $8.3 \pm 0.2$ & $8.4 \pm 0.2$ & $8.6 \pm 0.2$ \\
\hline & Zhongyou 821 & $7.7 \pm 0.3$ & $7.9 \pm 0.3$ & $7.6 \pm 0.34$ & $7.6 \pm 0.3$ & $7.4 \pm 0.2$ \\
\hline \multirow{2}{*}{$\begin{array}{l}\text { Lesion } \\
\text { length } \\
{[\mathrm{mm}]}\end{array}$} & Loras & $24.0 \pm 5.1$ & $17.6 \pm 5.1$ & $17.2 \pm 4.5$ & $14.6 \pm 3.3$ & $19.6 \pm 3.9$ \\
\hline & Zhongyou 821 & $6.5 \pm 1.7$ & $5.3 \pm 0.9$ & $4.4 \pm 1.3$ & $11.5 \pm 3.1$ & $9.7 \pm 1.9$ \\
\hline
\end{tabular}




\section{Danksagung}

Mein Dank gilt Herrn Prof. Dr. Andreas von Tiedemann für die Bereitstellung des Themas und die Unterstützung durch Anregungen aber auch Freiheiten bei der Durchführung der Arbeit.

Herrn Prof. Dr. Petr Karlovsky danke ich für die Übernahme des Korreferats.

Ebenso danke ich Herrn Prof. Dr. Heiko Becker für die Bereitschaft, dem Prüfungskomitee anzugehören.

Diese Arbeit ist innerhalb des Verbundprojektes "Mechanistic analysis of quantitative disease resistance in Brassica via associative transcriptomics“ entstanden und wurde von der DFG finanziert. Vielen Dank an die Kollegen aus dem MAQBAT-Projekt für die interessanten Diskussionen sowie die Bereitstellung der Daten durch Dr. Christoph Crocoll.

Herzlichen gedankt sei Evelin Vorbeck, Dagmar Tacke, Jutta Schaper und vielen anderen Phytopaths für die stete Bereitschaft zu Tipps und Hilfestellungen. Ein großer Dank geht an unsere Auszubildenden Isabella Reitz für den fleißigen Einsatz in Gewächshaus und Labor, sowie an Katharina Henze für die knifflige Quantifizierung der Pilz-DNA.

Vielen Dank an Maik Knobel, ohne dessen beständige und gute Pflege der Pflanzen die Gewächshausversuche kaum durchführbar gewesen wären. Ich danke Frank Gremmes und Holger Heitmüller für die Hilfe bei der Lösung technischer Probleme.

Dr. Anke Sirrenberg danke ich für die Unterstützung bei der Begutachtung mikroskopischer Aufnahmen.

Marco Winkler, Abteilung Molekulare Holzbiotechnologie und technische Mykologie, sei gedankt für die wertvollen Erklärungen über die Anfertigung von Paraffinschnitten am Rotationsmikrotom.

Meiner lieben Kollegin Xiaorong Zheng danke ich dafür, dass wir den Kraftakt Screening gemeinsam und im Einklang so gut gemeistert haben, und für die vielen themenbezogenen und anderen interessanten Gespräche, die wir geführt haben.

Für die Hilfe beim letzten Screeningdurchgang danke ich ganz besonders Nils Christiansen für seinen zuverlässigen und unermüdlichen Einsatz und die lustigen Momente zu später Stunde im Gewächsaus bei Flachwitzen und Pizza.

Merci beaucoup, Asmae Meziane, pour ton aide aux analyses au laboratoire qui ont demandé une persévérance particulière de ta part. II me faisait plaisir de travailler avec toi. Allen Kollegen einen besonderen Dank für die tolle Arbeitsatmosphäre, die gemeinsamen Diskussionen, die munteren Teepausen und auch die erlebnisreichen Unternehmungen außerhalb des Instituts. Es war eine spannende, erfahrungsreiche und lustige Zeit.

Mark Winter danke ich für das Encouragement.

Für hilfreiche Anmerkungen und Korrekturen im Text danke ich Xiaorong Zheng, Dörte Kruse, Volker und Moritz Wilch, und Gregor Welna.

Der größte Dank gilt meiner Familie und Gregor, die mir mit Wort und Tat zur Seite standen. Durch ihre Unterstützung, ihr Zureden und ihr Verständnis in mancherlei Situation haben sie zum Gelingen dieser Arbeit beigetragen. 


\section{Declarations}

I, hereby, declare that this $\mathrm{PhD}$ dissertation has not been presented to any other examining body either in its present or a similar form.

Furthermore, I also affirm that I have not applied for a PhD at any other higher school of education.

I, hereby, solemnly declare that this dissertation was undertaken independently and without any unauthorised aid.

Göttingen, December 2018

(Signature)

(Name in block capitals) 